$5.50 \%$

:

T. 8 .

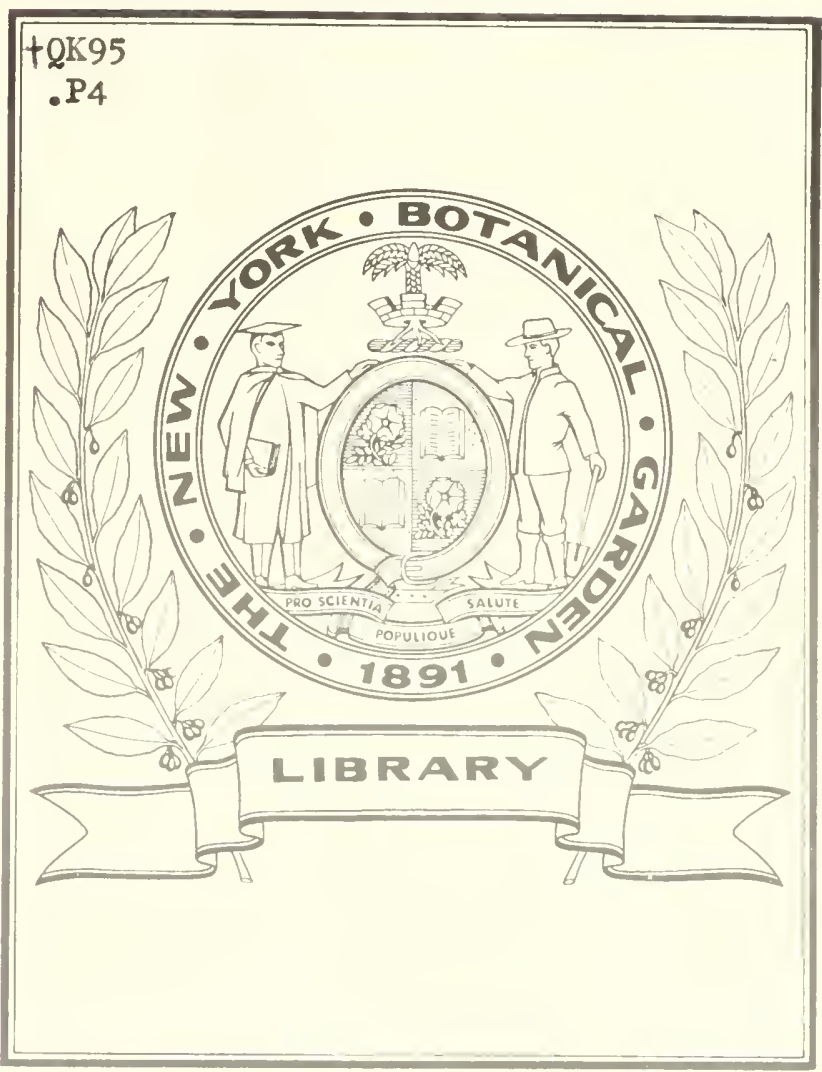

$\leftarrow \mathrm{AP}=\mathrm{Fl}$

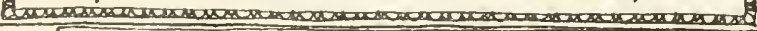

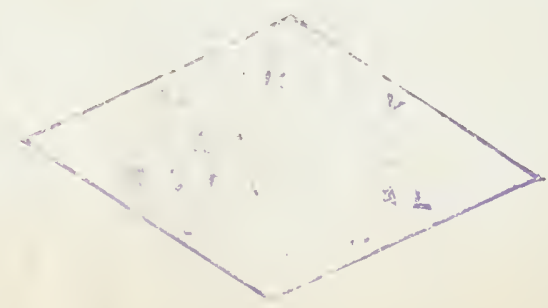


Eyse Ch: de Cosspizing. thidstberg 1839.

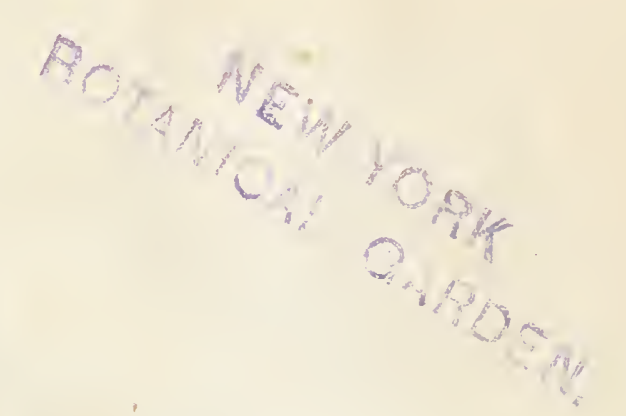


83

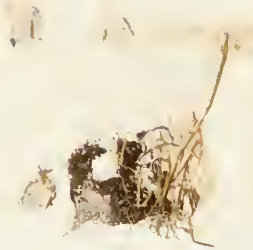




\title{
CIA VIS
}

\section{CLASSIUM, ORDINUM ET FAMILIARUM}

\author{
ATQUB
}

\section{INDEX GENERUM}

\section{REG NIVEGETABILIS.}

\section{Diagnostische Uebersichtstafeln}

\section{des \\ natürlichen Pflanzensystems.}

Nebst vollständigem Gattungsregister.

Von

Dr. C. J. P E R L E B,

lord. üfentl. Professor der Naturgeschichte und Botanik an der grossh. bad. Vuiversütät au Freiburg, plirector des ahadersiscken Nuturaliencabinettes u. botan. Gartens, mebr. gel. Ges. Mitgh

FREIBURG im Br., bei $\triangle$ D OLP II EMMER ING.

$\begin{array}{llll}1 & 8 & 3 & 8\end{array}$ 

Der langwierige Streit unter den Botanikern über den Vorzug des Sexualsystemes oder der natürlichen Methode ist gegenwärtig wohl als entschieden und geendet zu betrachten. Auch die Anwendbarkeit der letztern zum Unterrichte hat sich saitsam bewährt. Die in dem Wesen dieser Methode liegende Schwierigkeit, die classificatorischen Gruppen mit wenigen Worten und scharf zu definiren, läfst selbst zum Behufe der Erleichterung des Anfängers sich bis zu einem befriedigenden Grade überwinden oder umgehen. Es gibt hiefür verschiedene Nittel. Besonders hat man die von LAMArok eingeführten analytischen Kettentabellen zu diesem Endzwecke dienlich gefunden, und sie bieten in der That vielfache Vortheile dar. Doch mangelt ihnen jene bequeme Uebersichtlichkeit, welche der ältern, schon von $\mathbb{R}_{\Lambda \mathbf{Y}}$ gebrauchten Tabellenform eigen ist. In der vorliegenden Schrift habe ich daher wieder diese Form gewählt, diagnostische Tafeln der Classen, Ordnungen und Familien des natürlichen Pflanzensystems auszuführen. Der Entwurf derselben war zunächst nur für meine Zuhörer zu ihrer praktisch-botanischen Uebung bestimmt; eine geraume Zeit her damit angestellte Proben lassen mich aber die Hoffnung hegen, dafs die öffentliche Herausgabe auch in weiterm Kreise einem Bedürfnifs entsprechen werde.

Als Grundlage dieser meiner Arbeit behiclt ich dasjenige System bei, welches ich schon in meinem Lehrbuche der Naturgeschichte, Band I. - wovon der botanische Theil auch in besonderm Abdrucke unter dem Titel: Lehrbuch der Naturgeschichte des Pflanzenreichs (Freiburg, bei Fr. Wagner 1826) ausgegeben wurde, - aufgestellt habe, und welches sich in dem Hauptgange an Decaxdolne's System anschliefst, jedoch durch andre Umschreibung der Classen und durch Einführung ron cigenen Mittelgruppen („O rdnung en") zwischen den Classen und Familien selbstständig davon unterscheidet. Es ist demselben von vielen Seiten freundliche und ehrenvolle Anerkennung zu Theil geworden, während es freilich von einigen nur vornehm ignorirt oder auch stilischweigend benützt wurde*).

*) So namentlich diefs noch jüngst in dem II andbuch der Naturgeschichte ron Professor Burmeister in Berliu, l. Abth. (Berlin, bei Euslin 1836), in welchem Werke nicht weniger als 33 der von mir aufgestellien 44 Ordnungen der Pflanzen adoptirt sind, ohne dafs dabei irgendwo meines Buches oder meines Namens Erwähnung geschieht! 
Um des letztern Umstandes willen schien mir angemessen, an meinen damaligen Eintheilungen auch jetzt nichts Wesentliches zu ändern, obschon die binnen zwölf Jahren erschienenen Werke für Fortbildung des natürlichen Pflanzensystems, und die Ergebnisse meiner eignen seitherigen Studien mir allerdings vielen Anlafs dazu darboten.

Demnach wurden blos die im genannten Lehrbuche übergangenen oder inzwischen neu gebildeten Familien hier in dem Clavis eingeschaltet, aber zugleich mit einem beigesetzten $f$ bezeichnet, und durch Zerlegung der frühern Ordnung Squamiftorce, so wie durch Bildung der neuen Ordnung Pleurosperma wurde die Gesammtzahl der Ordnungen auf achtundvierzig vermehrt.

Im Uebrigen ist hinsichtlich der Einrichtung und Anwendung meines Clavis noch Folgendes zu bemerken.

Die lateinische Terminologie zog ich der deutschen vor, weil sie kürzer und bestimmter ist, bequemere Abbreviaturen zuläfst, und ja doch von Jedem, der Botanik studirt, eingeübt werden soll, wozu nun eben die Benützung eines solchen Clavis eine sehr passende Gelegenhcit gibt.

Die Diagnosen der Classen, Ordnungen und Familien beruhen zwar vorzugsweise auf den typischen Bildungen, aber sie würden unwahr und unpraktisch sein, wenn sie darnach ausschliefslich und mit voller Schärfe aufgestellt wären. Denn die natürlichen Gruppen fassen Bildungstypen mit freien Uebcrgängen nach allen Seiten zusammen, wodurch eine Versatilität der Charaktere entsteht, welche nicht unberücksichtigt bleiben darf. Daher ist in dem Clavis die Unbeständigkeit der einzelnen diagnostischen Merkmale gewöhnlich durch das Wörtchen plerumque angezeigt, oft sind auch die vorkommenden Verschiedenheiten selbst aufgeführt, oder es ist durch beigefügte Nebenkennzeichen das Bestimmen der Gruppe erleichtert. Hiemit geht nun freilich an vielen Stellen die formelle Präcision verloren, abcr es ist diefs (wie schon Eingangs erinnert wurde) ein auf der Wesenheit der natürlichen Methode beruhendes und für diese durchaus folgerichtiges Verfahren. Sogar schliefsen sich in Folge dessen die Charakteristiken gewisser coordinirten Gruppen wechselseitig nicht genau aus; allein formell ist dieser Uebelstand alsbald zu bescitigen, wenn man nur erwähṇtes Wörtchen plerumque wegdenkt, und die sich ergebenden reellen Anstände beim Pflanzenbestimmen werden grörstentheils schwinden, wemu man in solchen Fällen die Diagnosen der den zweifelhaften Gruppen, z. B. Ordnungen, untergeordneten Familien durchvergleicht. - Immerhin wird indefs der Clavis nicht in allen Fällen ausreichen; vermag der Anfänger mit Hilfe desselben von je zehn Pflanzen acht 


\section{$-\mathbf{v}-$}

sicher zu classificiren, so leistet das Hilfsmittel unstreitig soviel, als man nach der Natur der Sache erwarten kann.

Die beiden Register, welche dem Clavis beigegeben sind, werden von mehrfachem Nutzen sein. Das erste derselben enthält eine synonymische Angabe der bei verschiedenen Auctoren vorkommenden Classificationsgruppen, namentlich der Familien, und verstattet daher eine vielseitige Vergleichung meiner Eintheilungen des Pflanzenreichs mit andern. Das Gattungsregister dient dazu, jede Pflanzengattung, die man dem Namen nach kennt, in ihre Familie einzureihen, also insbesondre auch zur systematischen Anordnung von Herbarien.

Freiburg im Breisgau, 20. Juni 183\%.

$\mathbf{P b}$ 。 


\section{- VI
K $\ddot{\mathbf{a}} \mathbf{I} \mathrm{u} \mathbf{g}$}

\section{der in dem Clavis vorkommenden terminologischen Abkürzungen und Zeichen.}

achaen. = achanium

-adelph. $=$-adelphus ( $z$. B.

1 -adelph. = monadelphus)

adnat. = adnatus

adhor. = adhorens

aqual. $=$ acqualis

arstivat. = astivatio

aggreg. = aggregatus

alb. $=$ albumen

albos. = albuminosus

alt,$=$ alternus

anther. = anthera

anthod. = anthodium

arb. = arbores

arboresc. = urborescens

arbusc. = arbusculas

arill., arillat. = arillus,

arillatus

artic. $=$ articulatus

axillar. = axillaris

bacc., baccat. = bacca, baccatus

bract., bracteat. = bractea,

bracteatus

caduc. = caducus

c. add. = cum additamentis

cal. $=$ calyx

calc. = calcar, calcaratus

caps., capsular. $=$ capsula

capsularis

carnos. = carnosus

carp. $=$ carpellum

caryops. $=$ caryopsis

cirrh., cirrhos. $=$ cirrhus,

cirrhosus

coadun. = coadunatus

cohar. $=$ cohorens

column., columnar. $=$ columna, columnaris conm. = communis

comp. $=$ compositus

comnat. = comatus

cor. $=$ corolla

coriac. $=$ coriaceus

cotyl. = cotyledones

curv. = curvus, curvatus

decid. $=$ deciduns

defin. $=$ definitus

dehisc. $=$ dehiscens

dissep. $=$ dissepimentum

drup. $=$ drupa, drupaceus

dupl. = duplus, duplex

e. e. $=$ exclusis excludendis

ecalc. = evalcaratus

embr. = embryo

epipet. = epipetalus

erect. $=$ erectus

exalbos. = cxalbuminosus

exstip. = exstipulatus

exter. = cxterior

extrors. $=$ extrorsus

fascic. $=$ fasciculus, fascicu-

latus

$-f i d .=-f i d u s$ (z. B. $4-f i d .=$ quadrifidus)

fl. flor., - flor. $=$ flos, flores,

-florus ( $\approx$. B. 3-flor. =

triflorus)

fol. = folia

follic. = folliculus, follicular is

fruct. $=$ fructus

frut. $=$ frutices

frutesc. $=$ frutescens

hbae = horbae

herbac. $=$ herbaceus

hermaph. = hermaphroditus

indefin. = indefinitus

\section{is.}

iridehisc. $=$ indehiscens

inf. = inferus

inflor. = inflorescentia

insert. $=$ insertus

integerr. = integerrima (folia)

integr. = integra (folia)

inter. $=$ interior

interpet. = interpetiolaris

intrapet. = intrapetiolaris

intrors. = introrsus

irreg. = irregularis

lactesc. = lactescens

lateral. $=$ lateralis

limb. $=$ limbus

lob., lobat. = lobus, lobatus

loc., -locul. = loculi, -locularis

(ะ. B. 2-loc. = bilocularis)

longitud. $=$ longitudinalis

marcesc. $=$ marcescens

minut. $=$ nimutus

mucron. = mucronatus

nervos. $=$ nervosus

nodos. $=$ nodosus

mum. = mumerus

numeros. = numerosus

-oic. $=$-oicus ( . B. 1 -oic. $=$ monoicus)

oligosp. = oligospermus

opp., oppos. = oppositus

ovar. = ovarium

ovul., - ovul. $=$ ovulum, - ovu-

latus (ะ. B. 1-ovul. =unio-

vulatus)

parallelinerv.=parallelinervius

-part., -partit. $=$-partitus

(ะ. B.5-part. = quinque -

partitus)

parv. $=$ parvus 
pend., pendul. $=$ pendulus pepon. $=$ peponaceus perig., perigon. $=$ perigonizm persist. $=$ persistens person. = personatus pet., petal. petaloid. $=$ petalum ; petaloídeus placent. $=$ placenta pleiosp. $=$ pleiospermus plque $=$ plerumque plur. $=$ plura -poros. $=$-porosus punct. $=$ punctatus putam. = putamen pyren., -pyren. = pyrena; -pyrenus (z. B. 2-pyren. = dipyrenus)

racem., racemos. $=$ racemus, racemosus

rar., rariss. $=$ raro, rarissime recept. $=$ receptaculum

reg. $=$ regularis -rimos. $=$-rimosus ring. = ringens rot. $=$ rotalus samar., samarozd. = samara , samaroideus

sarment. = sarmentaceus

scand. = scandiens

sem. $=$ semen

semiinf. = semiinferus

somisup. = semisuperus

sempervir. $=$ sempervirens

sep. = sepalum

sess. $=$ sessulis

-sex. =-sexualis, (ะ. $B$.

$1-$ sex. $=$ unisexualis)

simpl. $=$ simple $x$

solitar. = solitarius

spadic. = spadicinus

-sperm. =-spermus ( . B.

1-sperm. = monospermus)

spic., spicat. $=$ spica, spicatus spinesc., spinos. $=$ spinescens, spinosus

stam. $=$ stamen

stigm. $=$ etigma

stip., stipulat. $=$ stipulae, stipulatus

styl. $=$ stylus suecul. = succulentus

suffrut. = suffrutices

sup. $=$ superus

terminal. = terminalis

tuberos. $=$ tuberosus

unguic. = unguiculatus

utric. = utriculus, utricularis

$v$. $=v e l$

vagin. = vagina, vaginans

valv.,-valv, valvar., valvat. $=$ valvula,-valvis (z. B. 2-valv. =bivalvis),valvaris,valvatus venos. $=$ venosus

verticill. = verticillus, verticil -

latus

volub. = volubilis

$\mathbf{0}=$ mullus

$ð=$ masculus

오 $=$ fomineus

$+=$ in des Verf. Lehrbuche der Pfanzenkunde nicht enthalten

$\because=$ zweifelhafter Stellung in Systeme. 


\section{Erklärung}

\section{der im Clavis vorkommenden Abkürzungen von Auctoren-Namen.}

Ach. oder Achar. = E. Acharius

Adans. $=$ Mich. Adanson Ad. Juss. = Adr. de Jussieu $A g .=C$. A. Agardh A. Rich. = Achill. Richard Bartl. $=$ Fr. Th. Bartling Batsch $=$ A. J. G. C. Batsch $B l .=$ C. L. Blume Bonpl. $=$ A. Bonpland Brongn. $=$ Ad. Brongniart Cambess, $=$ J. Cambessèdes Cass. $=$ H. Cassini

Cham. et Schl. $=$ Adelb. $v$. Chamisso und D. F. L. v. Schlechtendal

Corr. $=$ J. Correa de Serra $D C .=A . P$. de Candolle $D_{\text {esv. }}=$ N. A. Desvaux Don $=$ Dav. Don Dumort. $=$ B. C. Dumortier Ehrbg. $=$ C. G. Ehrenberg Endl. = Steph. Endlicher Fr. $=$ El. Fries

Gay $=$ J. Gay
Gray = S. F. Gray

Hedw. = J. Hedwig

Hook. $=$ W. J. Hooker

$J a c k=W . J a c k$

Juss. $=$ A. L. de Jussieu

Kaulf. $=$ G. F. Kaulfuss

$K t h .=C . S$. Kunth

Lapyl. = N. de la Pylaie

$L_{d l} .=J$. Lindley

Less. oder Lessg. $=\mathrm{Chr}$. $\mathrm{Fr}$. Lessing

Lestib. $=T$ Th. Lestiboudois

L. oder Linn. $=$ C. v. Linné

$L k_{0}=H_{\text {. }} \boldsymbol{F}$. Link

Lmrx. $=J . V . F$. Lamouroux

Lyngb. $=H$. Chr. Lyngbye

Mart. $=$ C. Fr. Ph. v. Martius

Mirb.$=$ C. F. Brisseau-Mirbel

Nees $=C$. G. Nees v. Esenbeck

Nutt. $=$ Thom. Nuttall

$P b .=$ C. J. Perleb

Pers. $=$ C. H. Persoon

Pet. $-T_{\text {. }}=$ A. Audebert du Petit - Thouars
Poit. $=$ A. Poiteau

Ponted. $=$ Jul. Pontedera

Presl $=$ C. B. Presl

Ray $=J_{o h}$. Ray

R. Br. $=$ Rob. Brown

$R c h b .=H$. G. L. Reichenbach

Rich. $=C l_{0}$ L. Richard

Roth. $=A$. W. Roth

Salisb. $=$ R. A. Salisbury

Schlchtd. = D.F.L.v. Schlechtendal

Schrad. $=$ H. A. Schrader

Spr. $=$ Curt Sprengel

St.Hil.=Aug. de Saint-Hilaire

Tourn. $=$ J. Pitton de Tourne-

fort

Turp. $=P$. J.F. Turpin

Vaill. $=$ Seb. Vaillant

$V_{\text {ent }} .=S t . P$. Ventenat

Wall. $=$ Nath. Wallich

Web. et M. $=$ Fr. Weber und D. M. H. Mohr

Willd. $=$ C. L. Willdenow. 


\section{Clavis Classium, Ordinum et Familiarum Regni vègetabilis.}

\section{A. Clavis Classium.}

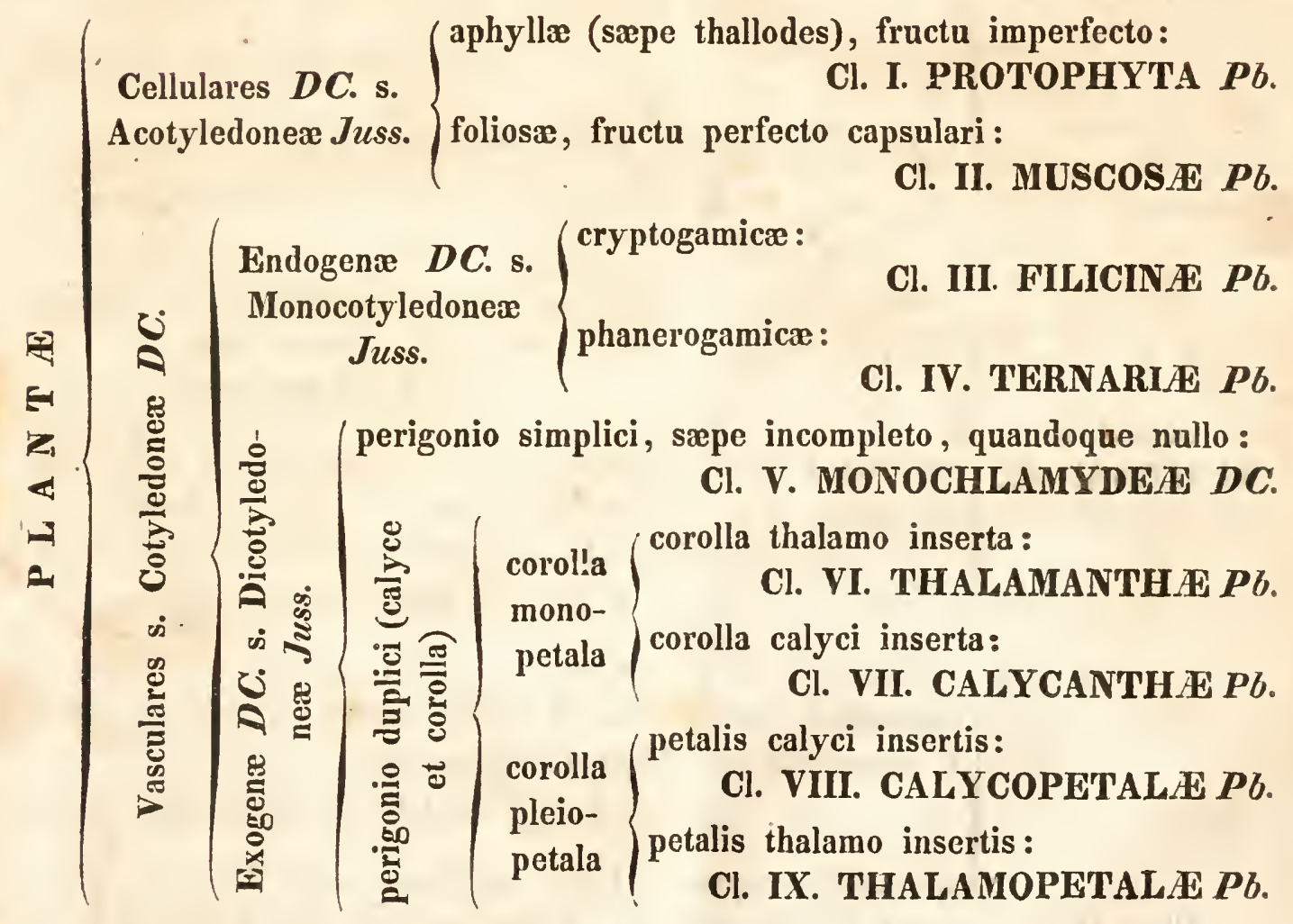




\section{B. Clavis Ordinum.}

\section{Class. I.
PROTOPHYTA Pb. \\ vegetabilia aêrea, nunquam vere vi- ridia $v$. in peridia $v$. ascos et hymenium in- clusis : \\ Ord. I. (1) FUNGI Linn., Juss. thallo explanato v. fruticuloso-ramoso; sporidiis in apothecia inclusis et sæpe superficialibus : \\ Ord. II. (2) LICHENES Achar. \\ vegetabilia aquatica plque submersa, saepe virentia: \\ Ord. III. (3) ALGA Linn., Juss.e.e., Roth.}

Class. II. MUSCOSE $P b$.

Class. III. FILICIN $P$ P (mycelio fugaci filamentoso e putredine exorientia v. in aliis plantis exanthematica; sporidiis nudis superficialibus

fronde lobata v. surculis foliatis; sporangiis non operculatis, valvatim $v$. tubulo $v$. poro dehiscentibus:

Ord. I. (4) HEPATIC Juss.

surculis foliatis; sporangiis operculatis plque urceolatis, rarissime valvatim dehiscentibus :

Ord. II. (5) OPERCULATA IV eb. et Mohr. s. MUSCI FRONDOSI Hedw.

sporangiis capsularibus 2-v. 4-valv., axillarib. v, spicat. v. racemos.; fol. simpI. v. compositis :

Ord. I. (6) DICLIDOPTERIDES Kaulf.

sporangiis capsular. 1 -v. plurilocal. non valvatis, plque dorso frondis insertis, rarius paniculatim dispositis; fol. frondosis, plque compos., vernatione circinato-involutis: Ord. II. (7) EPIPHYLLOSPERMAR Ray.

organis fructificationis duplicibus, in sacculos inclusis;'fol. plque simpl. v. $\mathbf{0}$ :

Ord. III. (8) THYLACOPTERIDES $P b$. 
staminib. cum pistillo non junctis

(perigon. $\mathbf{O}$ v. squamæformi ; florib. plque spadici insertis; - hbæ sæpe tuberos., rarius frut. $v$. arb. fol. alt. simpl. v. subcompos., nervos. v. venos., plque vaginantibus :

Ord.I. (9) SPADICIN $A$ Ag. c. add.Pb.

perigon. glumaceo; - plantæ culmiferæ plque herbacex, fol. alt. simpl. integerr. parallelinerv. vaginant.:

\section{Ord. II. (10) GLUMACEA Pb.} perigon. dupl., utroque 3-phyllo v. 3part. (rariss. abortu 2-phyll.); - caule plque herbac., fol. alt. simpl. integerr. nervos. v. rarius venos., vaginant.:

Ord. III. (11) TRIPETALOIDEA Linu. e. e. $\boldsymbol{P} b$.

perigon. perigon. simpl. v. dupl.; - caule arsimplici boreo $\dot{v}$. raro frutesc., plque simpliciss., v. duplici frondib. compos. parallelinerviis, in perfecto apice trunci congestis:

Ord. IV. (12) PALME Linn., Juss.

perigon. simpl. 6-fid. v. 6-petalo; plantæ sæpe bulbosæ, plque herbaceæ, fol. simpl. plque integerr. parallelinerviis, subvaginant. :

Ord. V. (13) LILIACE $\mathbb{E}$ Adans.e.e.Pb.

staminibus et pistillo varie connatis; - plantæ herbacex, quandoque prægrandes, saepe tuberosae, caule plque simpl., fol. simpl. integerrim., parallelinerv., subvaginantibus: Ord. VI. (14) GYNANDRA $A g$. 
"Gymnospermae"; - fl. 1-sex. plque amentac., perig. 0 ; ovar. apertis squamae -v. foliiformib., ovulis vere nudis; fruct. strobilac. v. rarius subdrupaceo; sem. albos., embr. saepe polycotyl.; - arb. v. frut. plque sempervirentes :

Ord. I. (I5) CONIFER E Juss. c. add. Bartl. ${ }^{*}$ ).
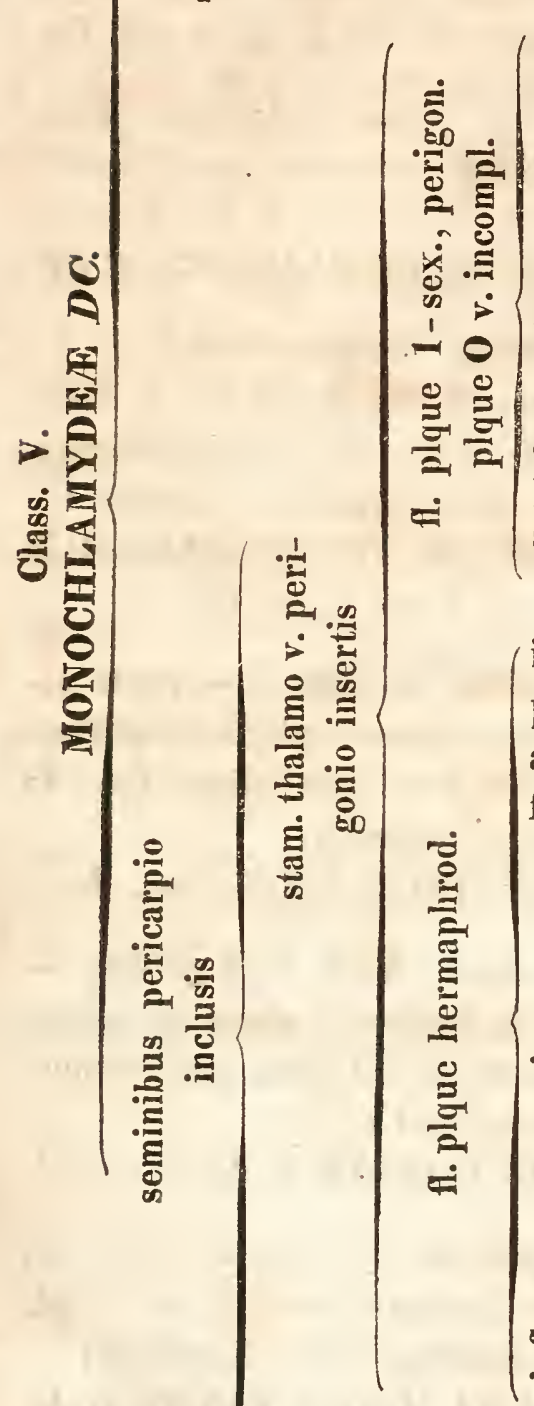

fl. amentac. 1-v. 2-oic. perig. O. v. incompl. plque squamaeform.; fruct. indehisc. sicc. v. subdrupac. 1-2-sperm. v. caps. 2-valv. polysperm.; sem. exalbos., embr. recto; - arb. v. frut. fol. alt. simpl. v. 0 :

Ord.II.(16) AMENTACE $\mathbb{E}$ Juss., Pb.

fl. plque 1-sex.; perig. 0 v. calyculiformi 2-6-lobo; fructib. utricular. v. baccat. v. capsular. saepe aggreg. v. involucro aucto carnoso receptis; sem. plque albos. embr. axili; - hbae, frut. v. arb. saepe lactesc. :

Ord. III. (17) UR'TICINE Bartl., Pb. infloresc. cylindrico-spadicin. v. amentaceo-spicat.; perig. 0 v. incompl.; ovar. sup.; sem. embr. inverso ad apicem albuminis carnos. v. farinacei; - hbae, frut. v. arb. fol. petiolat. vaginant. v. stipulatis :

Ord. IV. (18) PIPERINA Bartl.

( stam. thalamo v. basi perig. insertis; ovar. plque sup.; sem. plque farinaceo-albos., embr. plque curv. peripherico; - hbae, raro frut. v. arb., fol. saepe basi vaginant.:

Ord. V. (19) OLERACEA $A g$., $P$; stam. perigon. insertis, ovar. sup. v. inf.; sem. alb. 0 v. carnos., embr. recto; - arb. frut. v. hbae, fol. nec vaginant. nec. stipul.: Ord. VI. (20) LAUREOLINA Pb. stam. pistillo v. columnae centrali connatis; - hbae saepe aphyllae v. frut. foliati :

Ord. VIí. (21) STYLANDRA $P b$.

*) Die in meinem Lehrbuche aufgestellte Ordnung Squa miflor $x$ war zu umfassend, und es mufsten dann in den Familien derselben zu differente Bildungen vereinigt werden; naturgemäfser ist es, in obiger Weise mehre jener Familien als eigene Ordunngen (I - IV) $x$ unterscheiden, wobei nur noch die früher in die Cl. IV. Ord. I. gestellten Cyçdex, nenern Untersuchungen gemäfs, zu den Coniferæ gezogen wurlen. 
(ovar. et fructib. 4 ( raro 5 v. plurib.) 1 -spermis, distinct. v. connat.; - $\overrightarrow{\mathrm{a}}$ stam. 4 didynam. v. 2 v. 5 ; sem. alb. sub0 :

(cor. irreg.; stam. 4 didynam. v. 2; fruct. plque caps. 2 -v. 1 - (raro 4-8-) loc. polysperm. :

Ord. II. (23) PERSONA'T Adans e. e. Pb.

(stam. 4 didyn. v. 2 ; ovar. 1 ; fruct. 2-v. 4-sperm.: Ord. III. (24) PYRENACEA Vent. c. add. Pb.

ovar. $1 \mathrm{v}$. rar. 2 ( ovar. 1; fruct. 1-sperm. v. caps. 1-loc. polysperm., sem. columnae centrali affixis: Ord. I. (22) CYATHIN E Pb.

stam. 5 v. ovar. 1 ; fruct. caps. v. bacc. plque 2-v. cor. plque regul. rarius 4 3-loc. polysperm.:

(non di- Ord. V. (26) LURID $E$ Linn. c. add. $P Z$. dynamis)

ovar. 1 v. 2 ; coroll. saepe contorta; fruct. polysperm. capsular. sem. parietal. v. folliculis 2 , v. raro bacc. $v$. drupaceo:

Ord. VI. (27) PICROCHYLEA Pb.

stam. plque corollae lob. num. dupl. v. triplis (8, 10 -16 etc.); ovar. 1 ; fruct. plque pluriloc. polysperm. Ord. VII. (28) ATAXAE Pb.

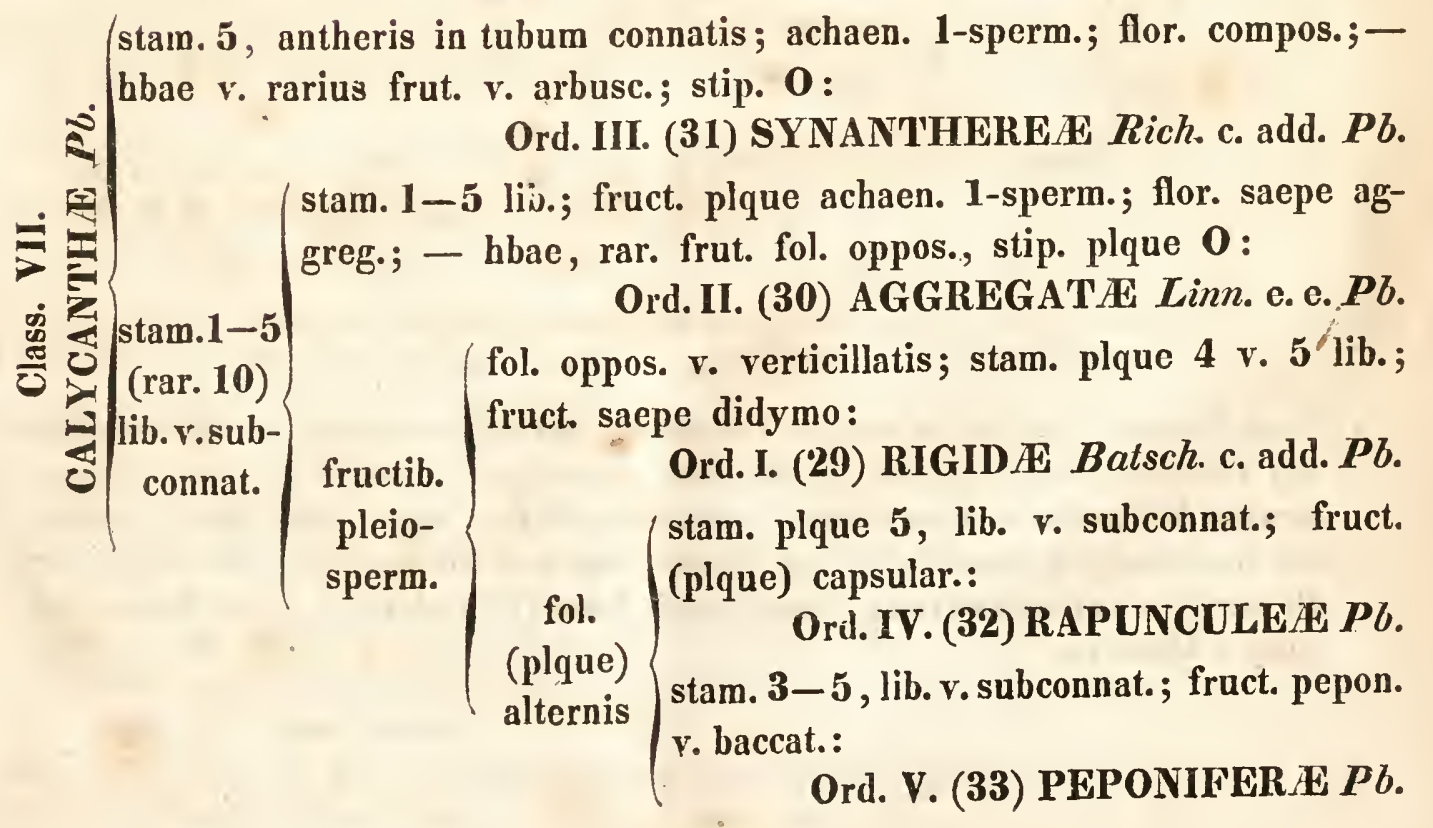




\section{$-0-$}

ovar. inf.; petal. et stam. num. defin., disco epigyno insertis; fruct. 2-12-loc., locul. 1-sperm., sem. pendul., albos.; - infloresc. plque. umbellata :

\section{Ord. I. (34) UMBRACULARIE Batsch. c. add. Pb.}

calycis lobo impari antico; ovar. sup. simpl.; fructu leguminos.
v. lomentaceo; sem. exalbos.; - fol. altern., plque pinnato-
compos., stipulat.:
Ord. III. (36) LEGUMINOSA Adans, Juss.

*) Diese Ordnung, welche in meinem Lehrbuche noch nicht enthalten und gröfstentheils aus Familien zusammengestellt ist, die dort übergangen wurden, bildet eine sehr naturgemäfse Mittelreihe in dieser Klasse, schliefst sich aber durch ihren Hauptcharacter, die wandständigen Saamenhalter, im Ganzen, und noch insbesondere durch einige ihrer Glieder (Smeathmanniex, Homaline vorizen Klasse an. 
perigonior. partib. num. quaternario: cal. 2-v. 4-sepal., cor. plque 4 - pet.; ovar. simpl.; fruct. siliquos. $v$. caps. v. bacc., sem. pendul.; fol. altern., stip. 0 :

\section{Ord. VIII. (47) CRUCIFLORA Pb.}

fruct. 'capsular. polysperm., sem. columnae centrali affixis; fl. regul. stam. lib. num. defin.; - hbae. v. suffrut. caule nodosoarticulato, fol. oppos. simplo, stip. $\mathbf{0}$ :

Ord. I. (40) CARYOPHYLLEA Juss. e. e. DC.

fruct. plque capsular. polysperm., sem. parietalib.; fl. saepe irreg.; stam. lib. v. raro num. defin. submonadelphis:

Ord. II. (41) VIOLARIA $P b$.

fruct. plque 3-loc. 3-sperm., caps., samara v. bacca; fl. reg. v. irreg., stam. lib. v. 1-adelphis:

Ord. IV. (43) TRIHILATA Linn. e. e.Pb.

fruct. plque multiloc., bacc. v. caps.; sem. angulo interno affixis; il. regul., cal. aestivat. imbric.; stam. poly-v. 1-adelph. v. lib.; - arb. v. frut., raro hbae, fol. exstipulat.:

Ord. VI. (45) HESPERIDEA Batsch c. add. $P b$.

ovar. unico v. quinis subconnat.; flor. vo irreg. cal. calcarat. v. reg. stam. num defin. lib. v. submonadelph.; fruct. simpl. bacc. v. caps. oligosperm. v. capsulis quinis 1 -spermis; hbae v. frut. fol. saepe stipulat.

Ord. VII. (46) SARMENTACE $\mathbb{E}$ Vent.c. add. $P b$. ovar.plque 5 v. 3, saepe subconnat.; fi. reg. v. irreg. non calcarat.; stam. rium. defin. plque lib.; fruct. submultipl. capsular., rarius drupac. v. bacc.:

Ord.III. (42) RUTARIÆ Pb.

ovar. plque numeros. verticillatis saepe connat.; fl. reg., aestivat. calycis plque valvata, corollae contorta; stam. num. indefin. v. defin. plque 1-adelph.; fruct. v. caps. simpl. pluriloc. v. capsulis pluribus verticill.; - fol. altern. stipulat.:

Ord. V. (44) COLUMNIFER E Linn. e. e. Pb. ovar. et carpell. plque numeros., rarius (coalitu v. abortu) unico ; stam. lib., plque num. indefin.; fruct. caryops., capsular. v. baccat.

Ord.IX. (48) MULTISILIQUOS A Batsch c. add. $\boldsymbol{P} b$. 


\section{Clavis Familiarum.}

\section{Class. I. PROTOPHYTA.}

in aliis plantis parasitici, plque ex earum epidermide erampentes; sporidiis demum nudis $v$. epidermide rúpta peridiatis:

Fam. 1. (1) CONIOMYCETES Fr.

filis simplicibus $v$. ramosis formati, sporidiis extus insidentibus $v$. intra apices resiculosos nascentibus:

Fam. 2. (2) HYPHOMYCETES Lk., Fr.

Ord. I. (1) e peridiis globosis (peridiis plque membranac. v. coriac., intus sporidia libera $\nabla$. FUNGI Juss.

primum clausis, demum varie de- peridiis plque lignoso-induratis, sæpe stromati innatis, ostiolo hiscent. formati, perforatis, intus nucleum et sporangia ascis inclusa forensporidiis repleti tibus:

†Fam. 4. (4) PYRENOMYCETES Fr. *)

e substantia fibroso-carnosa v. suberosa v. gelatinosa rarie formati, partim membrana fructificante (hymenio), sporidia ascis inclusa continente tecti :

Fam. 5. (5) HYMENOMYCETES Fr.

Ord. II. (2) LICHENES Achar. thallo explanato crustaceo, penitus adnato; apotheciis aut thallo immersis nucleiformibus, aut superficie insertis scutellatis :

Fam. 1. (6) CRUSTACEI Ag. foliaceo-lobato; apotheciis scutellatis :

Fam. 2. (7) LOBIOLATI Ag. partim in podetia erecta plque fistulosa producto, apotheciis subglobosis:

Fam. 3. (8) CEPHALOIDEI Ag. thallo toto fruticuloso-ramoso, ramis solidis teretibus $v$. plano-compressis laciniatis; apotheciis scutellatis:

Fam. 4. (9) RAMALINEL Ag.

-) Fries latte frūher diese Familie mit der vorlsergehenden verbunden, trennte sie aber selbst in der Folge, was unstreitig richtiger ist, und worin wir ihm nun gleiclıfalls folgen. 
gelatinos $x$, intus globulis v. filis articulatis farct $x$ :

Fam. 1. (10) NOSTOCHINE $A g$.

rigidæ, fragiles, corpuscula complanata plque lineis rectis circumscripta quadraugula crystalliformia sistentes, sæpe muco invoIutæ:

?† Fam. 2. (11) DIATOMACEAE Ag., Endl。

Ord. III. (3) fila nuda articulata simpl. v. ramosa, libera v. rarius conjugata v. reticulatim ALG E Linn. juilcta:

Juss. e. e. Ag.

Fam. 3. (12) CONFERVACEA Ag., Endl.*)

membranaceo-expansa, tenues, planae v. tubulosae, virides:

Fam. 4. (14) ULVACEAE Ag.

subcoriace 0 - frondosae, olivaceae, roseae v. purpureae, saepe caulescentes subfoliatac et fructibus spuriis instructae:

Fam. 5 (15) FUCACEAE Lmr.x., Pb.

\title{
Class. II. MUSCOSE.
}

fronde explanata lobata; sporang. globosis frondi immersis, tubulo v. poro dehiscentibus :

Fam. 1. (16) ENTOCARPAE Pl.

Ord. I. (4)
HEPATICA

Juss.

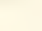

sporang. linearibus, subbivalv., columella centrali:

†Fam. 2. (17) ANTHOCEROTEAE Nees. nata aut caules-

cente foliata; spo- sporang. sub receptaculo communi pedunculato aggregatis, rang. $2-4-8-\{$ rarie deliscentibus :

valribus

(Fam. Valiate

$\mathrm{Pb}$. Lehrb.)

†Fam. 3. (18) MARCHANTIACEAE Nees, Endl.

sporang. pedunculatis quadrivalvibus:

†Fam. (15) JUNGERMANNIACEAE Nees, Dumort.

\footnotetext{
Ord. II. (5)

OPERCULA

TA $I$ I eb. et

sporangiis deliscentib. in valvulas 4 apice operouli ope connexas:

†Fam. 1. (20) ANDREAEACEAE Nees. Mohr.

DOSI Auct.)

sporangiis urecolatis, operculo plque circumscisse dejecto:

Fam. 2. (21) BRYACEAE Bartl.
}

*) Eine kleine eigenthümliche Fanliie, deren richtige Stellng im Systeme noch ztreifelhaft ist, bilden dic († Fam. 13) Charace Rich. - (Caule articulato, verticillatim ramoso, sporangiis majovibus spiraliter strintis et globulis minoribus cinnabarinis axillaribus.) 


\title{
Class. III. FIL I C I N AE.
}

Ord. I. (6)
DICLIDOP'IE-
RIDES Kaulf. in

caule simplici, folio plque solitario simpl. v. com
in spicas nudas distichas v. paniculas collectis :

Fam. 2. (23) OPHIOGLOSSEAE RBI.

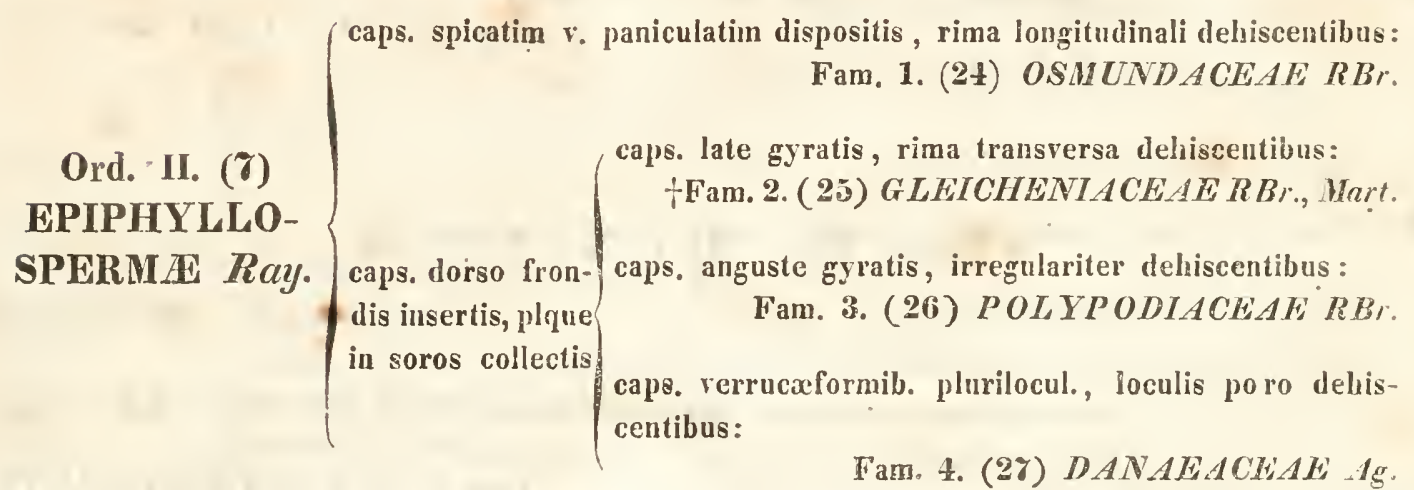

\begin{abstract}
caule repente $r$. subnullo, fol. subulatis $v$. ellipticis; sporangis basa. libus, globosis :

ord. III. (8)
THYLACOPTE-
RIDES $P$ b.

caule plque erecto, articulato, articulis vaginato et plque verticillatim ramoso; fol. 0 ; sporangiis ad spica terminalis receptacula peltata adnatis: Fam. 2. (30) EQUISETACEAE DC.
\end{abstract}

) Is ö̈tes Linn. wird besser von den Marsileaceen getrennt und als eigene Familie betrachtet; Bartling, v. Martius u. A. unterseheiden selbst noch weiter:

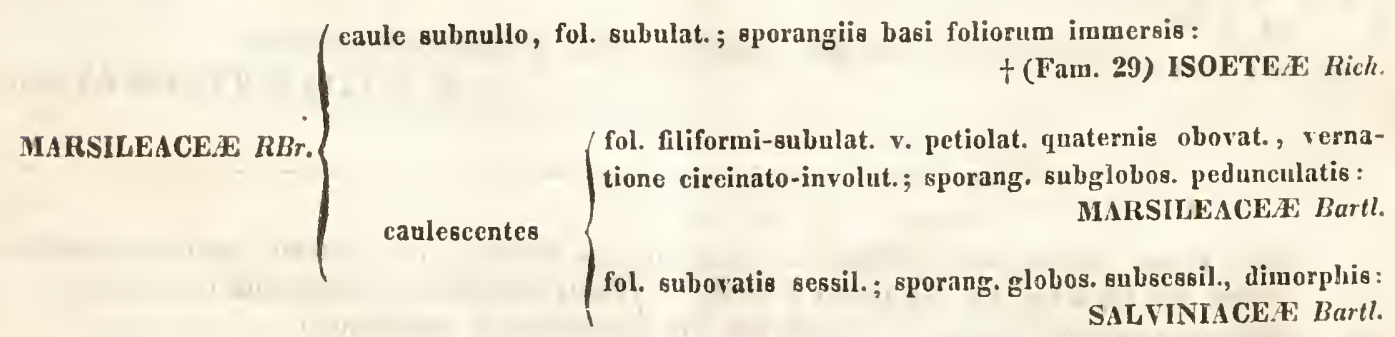




\section{Class. IV. TERNARIAE.}

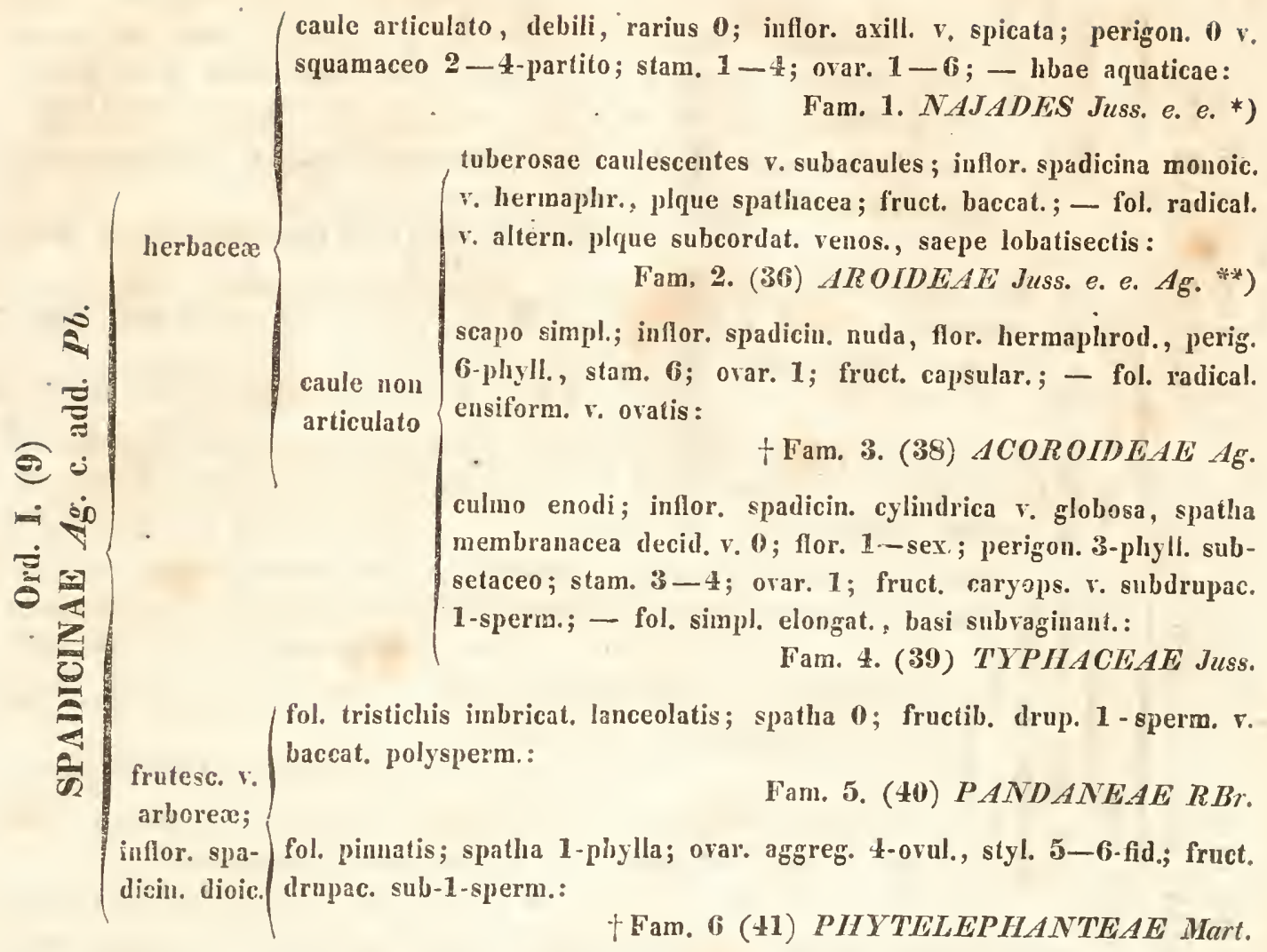

-) Die Naja des Juss. sind eine ungehôrige Verbindung von zum Theil sogar dicotyledonischen Pflanzcn ; es müsscn daher unterschicden werden :

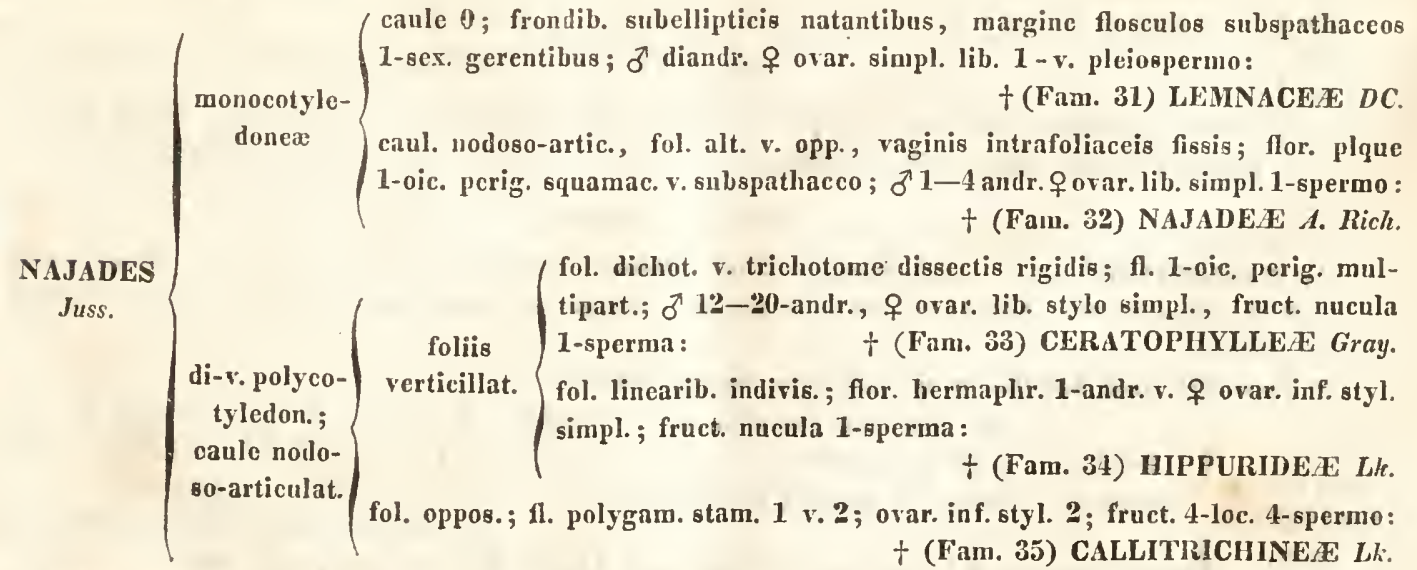

•) Als kleine hittelgruppe zwisclsen den Aroidec und $P$ andaneen sind zu betrachten die ( + Fam. 38) CYClanthe E Poit., Mart. - (caule herbac. r. sublignoso, fol. bifidis; spatha 4.plyylla.) 


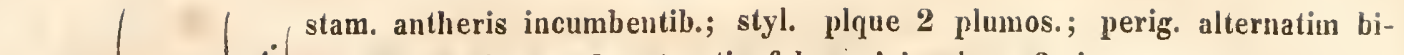 valr.; - culmo nodos. tereti ; fol. vagini s plque fissis : \\ Fam. 1 (42) GRAMINEAE Juss. \\ $:$ stam. antheris erectis; styl. 1, stigm. 3 v. 2; perig. 1 -valv.; sem. erect. embr. extra alb. hilo proxim.; - cnlmo plque enodi tereti v. 3-quetro; folior. vaginis plque clausis: Fam. 2. (\$3) CYPERACEAE DC. \\ ovar. plurib. axi comm. adnatis, 1-stylis; stam. 1; perigon. 0; fruct. utriculos. ; - culmo enodi filiformi; fol. radical., setaceis: \\ † Fam. 3. (44) CENTR OLEPIDEAE Desv. \\ - (llor. plque dioic.; stam. 3 v. raro 2 , antheris 1 -loc. peltatis; caps. 3 -loc. 3-sperm. (v. abortu 1-sperm.), sem. invers., enbr. extra alb. ab hilo remot; - culmo saepe nodos, f fol. vagin. plque fissis: \\ † Fam. 4. (45) RESTIONEAE Bartl. *) \\ flor. plque hermaphr.; stam. 6 v. rarius 3 , antheris 2 -loc. terminal.; caps. 3-loc., locul. poly-v. 1 -sperm., v. 1-loc. 3-sperm., sem. ereç, embr. in basi alb. incluso;-culm. nodos., fol. vaginant. \\ Fam. 5. (16) JUNCEAE DC.

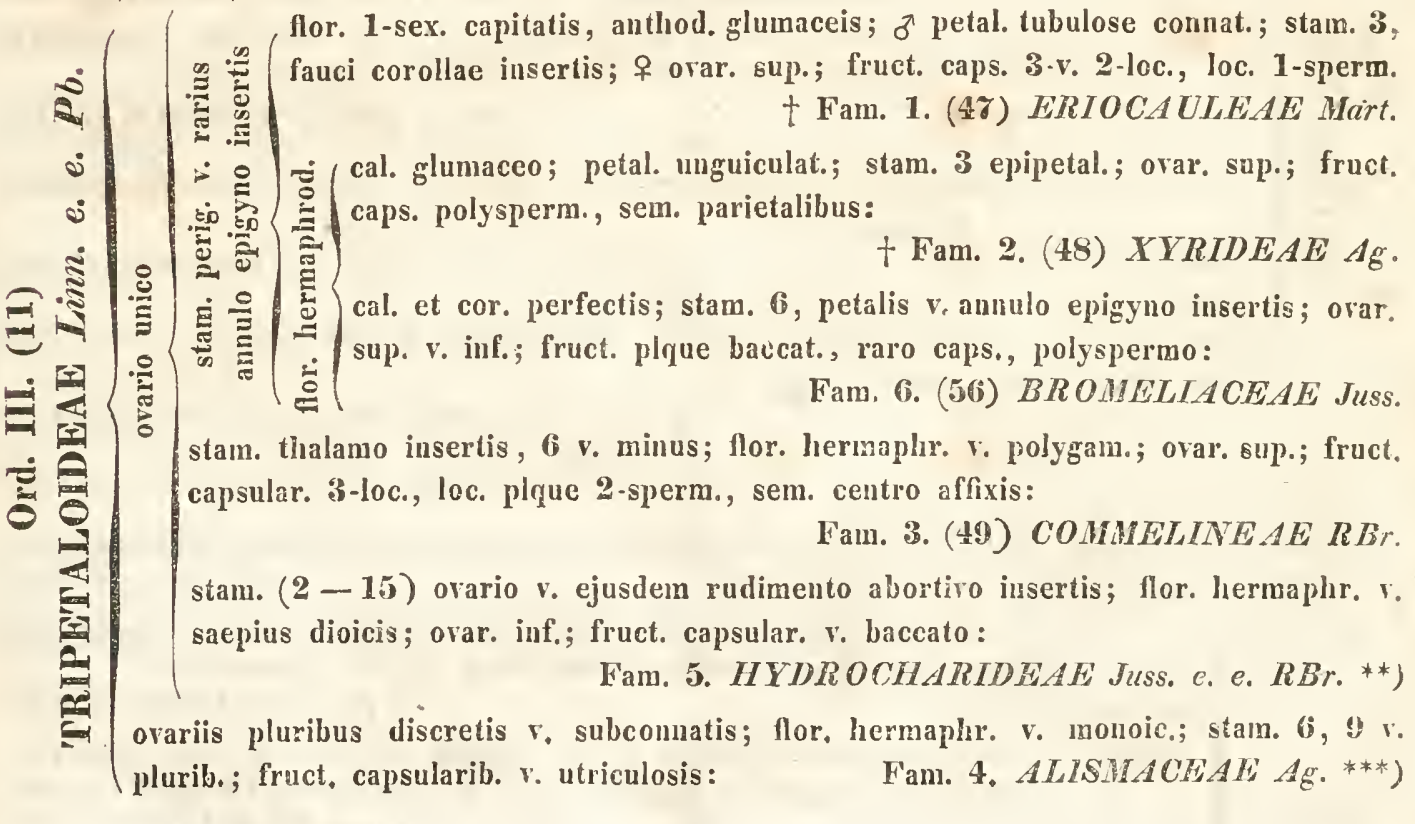

*) Dic RestiaCe $R$ e RBr. eind richtiger in dic kleinern Familien Centrolepidea, Restionea, Eriocaulex und Xyrideæ getheilt worden, wovon die zwei letztern in die folgende Ordnung gehören.

*) LINK hat diese Familie in drei kleinere zerlegt, nămlich :

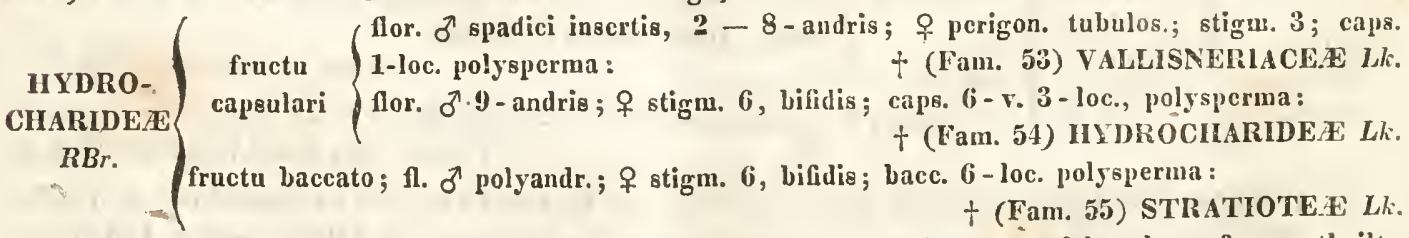

"..) Auch diese Familie würde viclleicht richtiger, nach Rucuskv's Vorgange , folgendermafsen zerthcilt: 


\section{$-13-$}

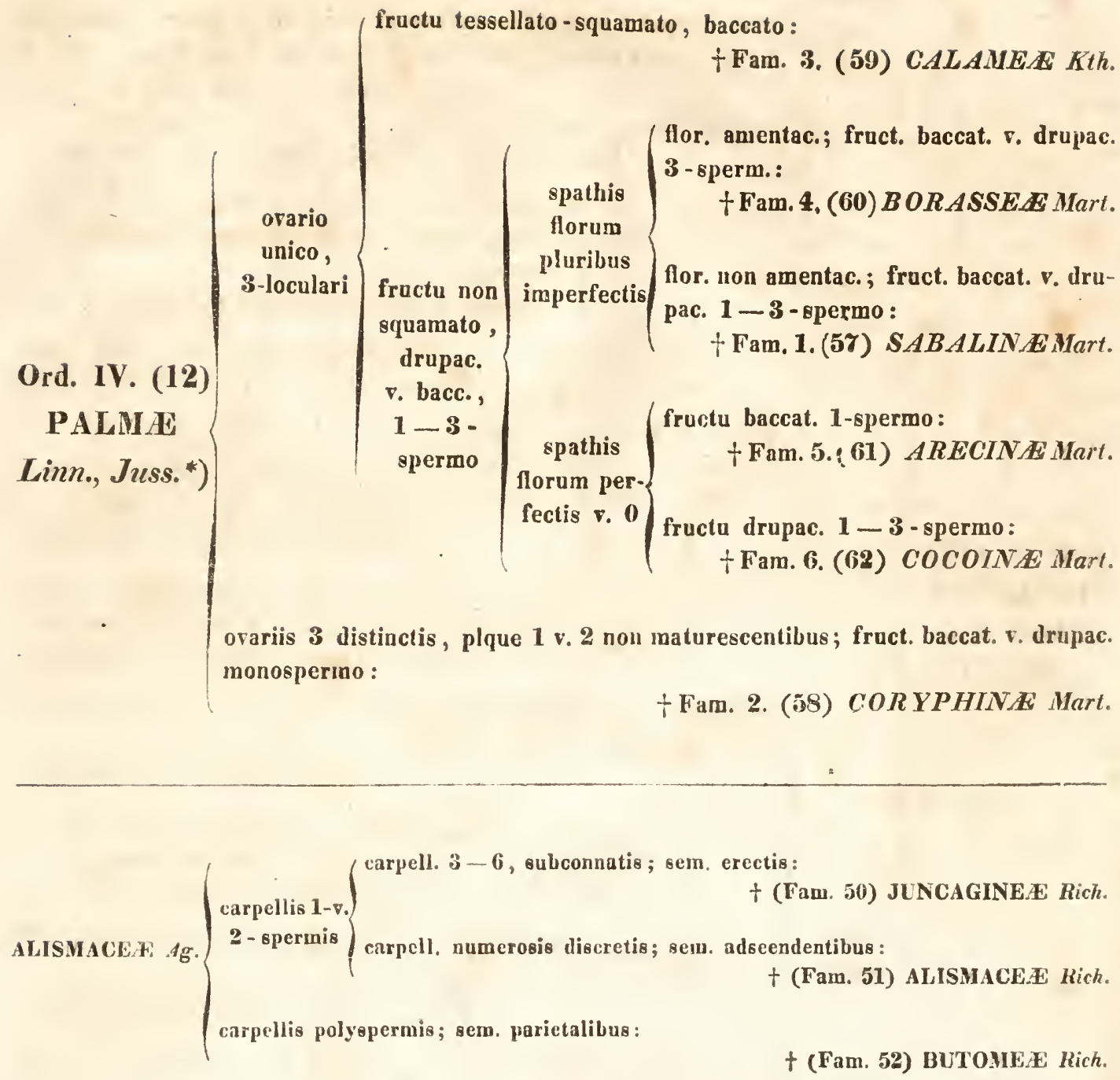

-) Dic Eiutheilung der Palmen in mehre Familien beruht auf so geringen Unterschieden, dafs ich in meinem Lehrbuche sie nicht annahm; indefs wird sie durch die aus ihr hervorgehende Symmetrie in der systematischen Gliederung empfohlen und durch die Analogie der Eintheilung verschiedener andern Ordnungen, wie namentlich zunächst der Liliaceen, hinlänglich gerechtfertigt. 
(fruct. (plque) baccato subgloboso; flor. saepe parr., quandoque abortu 1-sex.; stam. plque 6; styl. 1, stigın. saepe 3-fid. v. styl. 3 (rar. 4); - hbae, v. frut. saepe subvolub., raro arb., fol. quandoque reticulato venosis:

Fam. 2. (64) ASP ARAGEAE Juss, e. e. Ag. ${ }^{*}$ )

Ord. V. (13) LILIACEAE Adans e. e. Pb. ${ }^{* *}$ ) ovario

supero

ovario infero perigon. irreg. 6-fid., ante et post anthesin convoluto; stam. 3-6: styl. 1, stigm. simpl.; hbae aquat., rad. fibrosa:

†Fam. 1. (63) PONTEDERIACEAA.Rich.

caps. simplici sem. globos. $\nabla$, angulosis, testa fragili 3 -loc. perigon. atra: Fam.4.(66) $A S P H O D E L E A E$ fruct. 3-valv. (plque) Juss., $A g$.

regulari; sem. plque plano-compressis, testa stam. 6 non atra:

Fam. 5. (67) CORONARIAE Ag. capsulis ternis subconnatis, intus dehiscentibus; perigon. reg.; stam. 6; styl. 3 lib. v. partim counat.:

Fam. 8. (71) COLCHICACEAE DC.

flor. dioic.; caps. foliaceo.compress.; - frut. v. hbae, sæpe tuberos., caule volub., fol. plque reticulato-venosis;

Fam. 3. (65) DIOSCOREACEAE RBr. (fruct. caps. indehisc. v. subbaccat.; sem. (placent. (am. 6; centralib. affix.) rostrat., testa crustacea atra: ol. in- $\quad$ Fam.6.(68) HYPOXIDEAE RBr. tegr., omnibus fruct. caps., raro bacc.; sem. (plac. centr. aff.) radical. non rostrat. nec testa atra indutis:

Fam. 7. (69) AMAR YLLIDE'AE RBr.

flor. herstam. 6 v. 3, hisque perigonii lacin. interiorib. oppos. , maphr. anther. intrors.; styl. 1 stigm. iudivis.; - fol. caulin. alt. equitant.

† Fam. 9. (72) HAEMODORACEAE RBr. stam. perig. lacin. inter. oppos., anther. trans. versim dehisc.; orar. 3.alato; caps. polysperm., sem. minutis :

stam. 3 †Fam. 10.(73) B URMLANNIACEAE Bl.,Lindl. stam. perig. lacin. exteriorib. oppos.; anther. extrorsis; styl. 1 stigm. 3 -fid.; caps. polysperma: Fam. 11. (7t) IRIDEAE Juss.

-) Die frühere Trennung der $\Lambda$ sparage und $S$ milace ist unhaltbar.

*) 0 b die kleine Familie ( + 70) TACCEA Presl. auch in diese Ordnung gehöre, ist mir zweife!haft. Ihre Diagnose wäre: Hbae permagnae fol. radical. petiolat. plque pedatipartitis v. pinnatifidis; $f$ l. hermaphr.; ovar. inf.; stam. 6 , filam. petaloideo-dilatatis; fruct. bace. polysperm. sem. parietalibus. 
stam. cum stylo non connatis ; fol. magnis inte-

Ord. VI. (14) GYNANDRA $A g$. gerr., venis obliquis parallelis

stam. plque 6, saepe nonnullis sterilib.; - hbae praegrandes, saepe arboriformes:

Fam. 1. (75) MUSACEA Juss, DC. stam. fertilib. 2 et saepe tertio sterili:

† Fam. 3. (77) APOSTASIEAE Lindl. stam. fertili unico, caeteris petaloideis; stylo saepe intra sulcum filamenti recepto:

Fam. 2. (76) SCITAMINEAE Lirn. e.e. Spr. *) staminum filamentis cum stylo penitus in columnam (gynostemium) connatis, anthera 1 จ. rar. 2 ; fruct. caps. 1-loc., sem. numeros. minutis: Fam. 4 (78) ORCHIDEAE Limn., Juss.

\section{Class. V. MONOCHLAMYDE $A$. **)}

Ord. I. (15) CONIFERA Bartl.

(arb. caudice simplicissimo elato $\nabla$. abbreviato; fol. in ejusdem apice congestis, paucis sed magnis, frondosis pinnatis; inflor. amentac.; fruct. strobilaceo :

Fam. 1. (79) CYCADEAE Pers., Rich.

(flor. amentac.; 웅 ovariis squamaceo-apertis, seminib. binis; arb. $\nabla$. frut. ra- fruct. strobil. $\nabla$. galbulaceo:

mos., fol. simpl. $\quad$ f Fam. 2. (80) CEDRINAE Pb. plqne acerosis, flor. solitar.; 우 ovar. et fruct. solitar. 1-sperm., cupula saepe abortivis farnosa subinclusis drupaceis:

imbricatis $\quad$ Fam. 3. (81) WAXINAE Rich.

Ord. II. (16) AMENTACEA Juss., PZ. arb. aphyllae, ramis articulatis, vaginatis:

† Fam. 1. (82) CASUARINEB Mirb.

fruct. inde- (ovar. 1 - ovulat, ovulo erecto; fructib. solitar. subhiscent. $\quad$ drupaceis : plque 1-sperm.

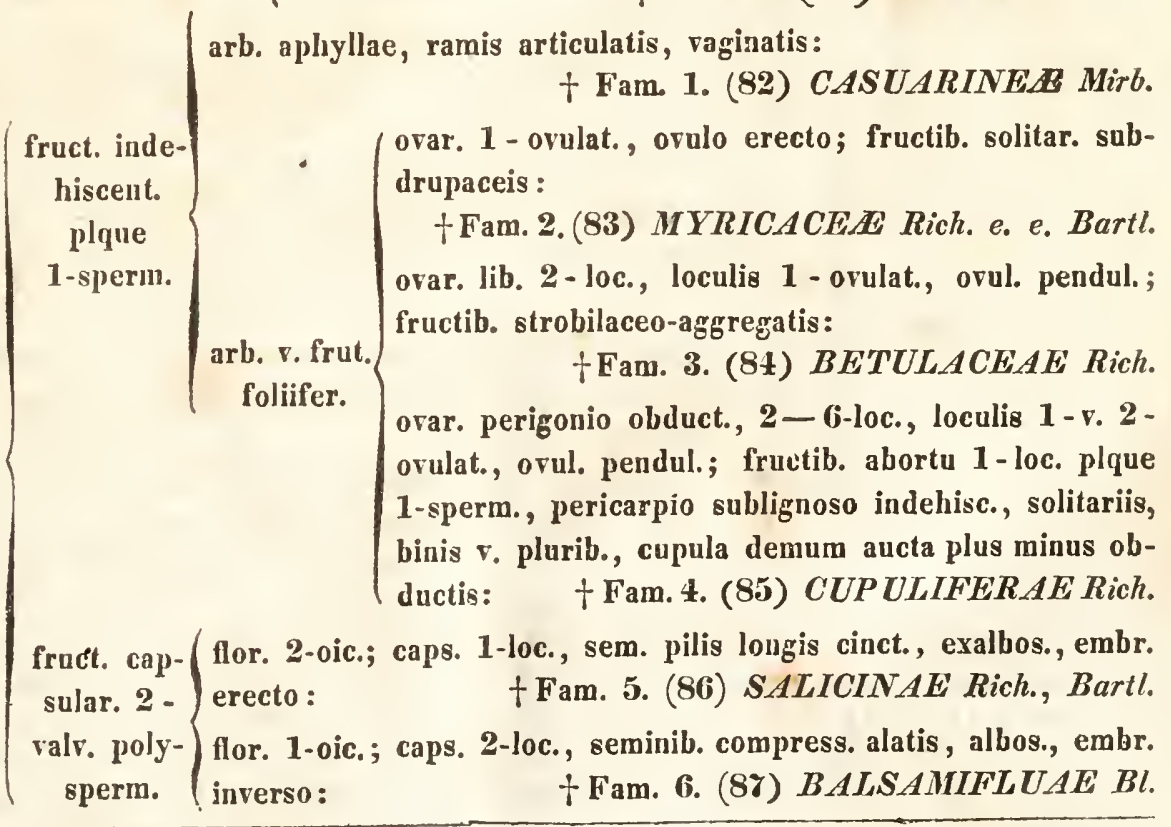

-) Mehre Auctoren theilen diese Familie in zwci, nảmlich :

SCITAMINEE ( anthera 2-loc., loculis remotis:

$\mathbf{S p} r$.

anthera limidiata 1-loculari:
AMOME E Rich. e. e. Bartl. (SCITAMINEE $R B r$.)

CANNACEA RBr. (MARANTACEA Lindl.)

*) Ueber den Bestand und die Grenzen dieser Klasse walten grofse Zweifel ob, zu deren kritischer Erledigung aber auf keinen Fall hier der Ort wäre. Auch finden sich, zumal in den zwei letzten 
flor. hermaphr. (raro abortive polygamis); perig. 5-r. 4-fid. persist.; stam. perig. lobis oppos.; ovar. 2 - loc., loc. 1 -ovulat.; stigm. 2 ; fruct. indelisc. membranac. v. drupac. 1 - . 2 -loc., sem. pendul.; - arb. $\mathbf{v}$, frut. fol. alt. simpl. scabris:

+ Fam. 1. (88) ULMACEAE Mirb.

ovar. et fruct. 1 - loc. polysperm., apice foramine magno dehisc.; sem. arillat. parietalib.; flor. dioicis, racemosis; - hbae fol. alt. pinnat.:

2 + Fam. 2. (89) DATISCEAE RBr.

Ord. III. (17) URTICIN $\mathrm{E}$

Bartl. c. add. Pb. *)

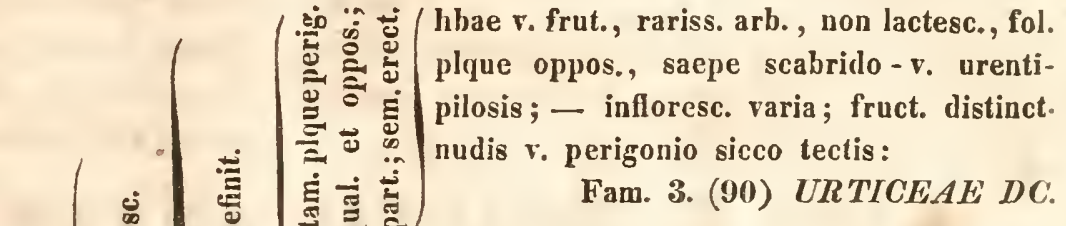
arb. v. frut., rarius hbae, lactesc., fol. plque alt.; - flor. aggreg.; fructib. in receptaculo aucto carnoso uidulantibus r. perigonio carnoso inclusis:

Fam.4.(91) ARTOCARPEAE DC., RBr. perigon. 0 ; stam. 1 ; 9 styl. incrassato, stigmate lateral. papilloso; sem. pendul.; - arb. v. frut. non lactesc., fol.alt. † Fam. 5. (92) PLATANEAE Lestib. stam. num. indefin., receptac. comm. calyciforni insidentibus; + ovar. 1-orul., stylo simpl, laterali v. basilari; fruct. receptac. aucto carnoso inclusis $\nabla$. immersis; sem. pendulo; - arb. v. frut. fol. oppos. pellucido-punctatis :

+ Fam. 6. MONIMIACEAE Juss. **)

antheris bilobis transversim dehisc., stam. 2 v. 5; 우 ovar. 2-ovul., basi cupula cincto, stigm. sess. 2-4-dentat.; fruct. drup. 1-sperm. sem. pendul.; - arb. v. frut. fol. alt. simpl.

+Fam. \%. (05) STLLAGINEAE Ag.
\end{abstract}

ovar. et fruct. e carpellis plque 3 connatis tricocco, rarius e 2 r. plurib., coccis sub integumento tenui v. carnoso elastice 2-valvib., sem. solitar. v. binis arillat. ab axe pendul.; perig. $2-6$-fil. v. 0 , additis saepe appendicibus petaluid. r. glandulos.; stam. num. defiı. v. indefin.; stigm. diris.; — arb., frut. v. hbae, quaedam lactesc., fol. alt., rariss. oppos. v. 0 :

? Fain. 8. (96) EUPHORBIACEAE Juss.

Klassen (Calycopetalx und Thalamopetala) aufser vielen einzelnen apetalischen Gattungen und Arten manche Familien, (wie z. B. Terebinthacea, Juglandec, Combretacea, Sanguisorbeæ, Chimonantheæ, Onagrariæ, Halorageæ, Ceratophylleæ, Hippuridex, Callitrichinex, Paronychiex, Samydex, Büttneriacex, Ranuneulacex etc.), welche ausschliefslich oder doch in grofser Anzahl solche Pflanzen enthalten, die durch Mangel der Petala monochlamylisch erscheinen, ihrer Gesammtrerwandtschaft nach jedoch richt aus jenen Klassen entfernt und in diese gestellt werden können. Für dcı Anfänger, welcher derartig abnorm gebildete Pflanzen im Systeme aufzufinden sucht, macht diefs freilich oft betrāchtliche Schwierigkeit. Eben, um auf die Familien, in denen dieses am hăufigsten statt findet, hinzuweisen, habe ich solche hier oben namhaft gemacht, damit vorkommenden Falles die Charaktere derselben nachgesehen werden mögen.

(Note ') und "•) 8. folgende Seite.) 
orar. $2-4$, basi partim connatis, singulis $1-v$. pauciorulat.; stam. 3, 6 v. plus. hypogyn.; fruct. caps. apice dehisc. v. drupac., sem. adscend.; - hbae aquat. v. palustr. fol. alt, v. radical., petiolis vaginae intrapetiol. fissae adnat.:

flor. perig.

† Fam. 1. (99) SAURUREAE Rich. †)

Ord. IV. (18) PIPERIN Bartl. c. add. Pb. nullo

ovar. simpl.

stam. 2, 3, raro plur., filam. orario adhaerentibus; fruct. subbaccat, sem. erecto; - hbae $v$. frut. fol. oppos., verticill. v. alt., petiol. basi vaginant.:

1-ovul.

Fam. 2. (100) PIPERACEAE Rich.

stam. 1 v. 3 connatis; fruct. drup., sem. pendulo; frut. fol. oppos., petiol. inferne in raginam cum stipulis parris conuat.:

+ Fam. 3 (101) CHLORANTHEAE RBr.

flor. amentac. perig. $4-8$-fid.; stam. 1; ovar, 1-loc. pluriovulat.; fruct. caps. 2-3-ralv., placent. parietal. 1-3-sperm., sem. pendul; - arbusc. v. frut. fol. alt., stip. parris caducis :

Fam. 4. (102) LACISTEMEAE Mart.
Ord. V. (19)

OLERACEA

Ag., c. add. Pb. stam, perig. iobis oppos. eorumque basi insertis; caryopsi perigonio, quandoque demum baccato, tecta: Fam. 1. (103) CHENOPODIEAE

non v. DC. e. e., Bartl.

parce stam. aut perig. lobos num. excedentibus aut bractea- totidem et iisdem tunc alt.; fruct. baccat, plque tis plurilocul., loc. 1-sperm.:

† Fam. 2. (10t) PHYTOLACCEAE RBr. Bartl.

bracteis coloratis plque flores inrolucrantibus; stam. thalamo insert., perig. lob. oppos. :

Fam. 3. (10ذ) AMARANTACEAE Juss. e.e.RBr. folior. petiolis basi raginant. v. vaginis intrapetiolaribus; fructu caryops. saepe trigona, quandoque perigonio tecta:

Fam. 4. (106) POLYGONEAE Juss. ovario iuf.; florib. monoic.; fruct. capsular. alato polysperm.;-fol. stipulat. alt., plque oblique cordatis:

Fam. 5. (107) BEGONIACEAE Bompl.

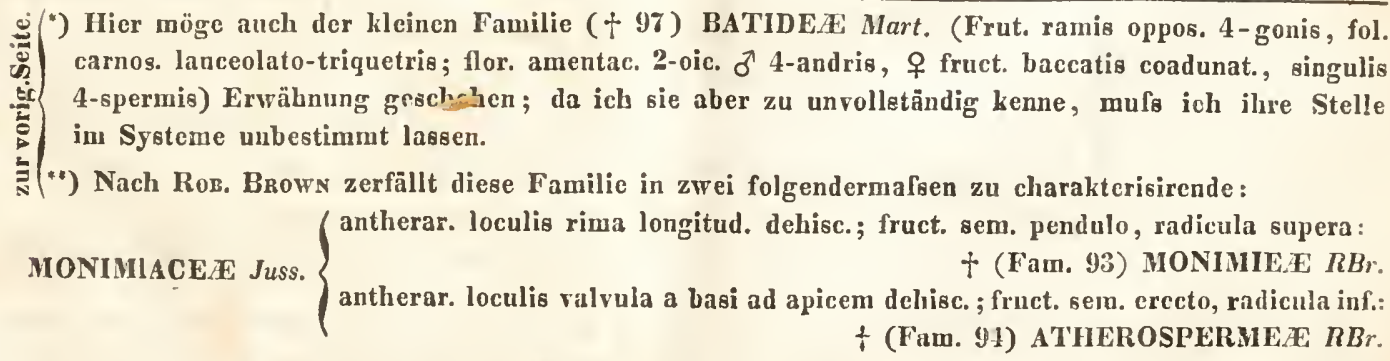

†) $\mathrm{Ob}$ die + Fam. (98) PODOSTENEE $L k$, neben die S a u r r e a zu stellen sey, bedarf noch 
ovario

infero

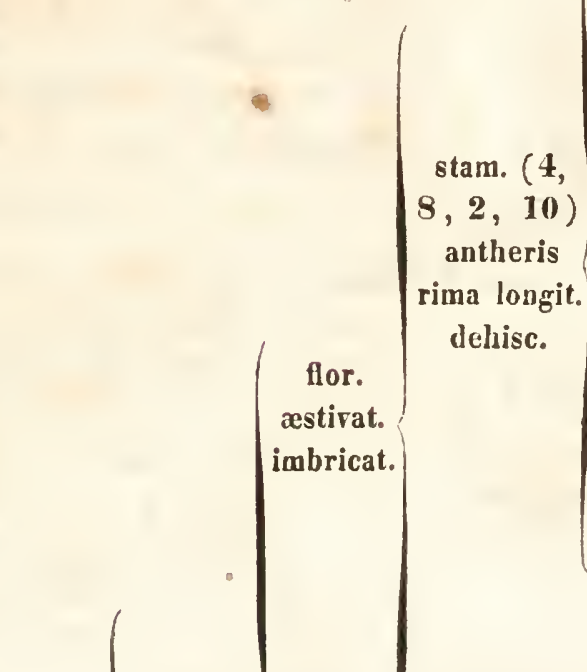

Fam. 2. (109) SANTALACEAE RBr. stam. plque 10; fruct. (abortive) 1 - sperm. subdrupac.; sem. exalbos., ootyled. spiraliter convolutis; - arb. v. frut. fol. alt. v. opp. integr.: Fam. 3. (110) MYROBALANEAE Juss. fruct. nucamentac. $\ulcorner$. drupac. 1 sperm., sem. pendulo; - frut. $v$. raro hbae fol. alt. $v$. opp. integerr.: Fam. 8. (115) THYMELAEAE antheris erectis Juss.

fruct. capsular. 4-loc. 4-valv., locul. 2-sperm.; - frut. sempervir. fol. seriatim imbricatis integr.: †Fam. 7. (114) PENAEACEAE $\boldsymbol{R B r}$, $\boldsymbol{K} t h$.

orar. stipitato ; fruct. capsular. 2valv. 2.sperm., valrulis seminiferls; - arb. fol. alt. integr.: †Fam.5.(112) AQUILARINEA antheris incumbentib.

stam. 6, 9, 12, antheris valvatim dehisc.; - arb. fol. alt. r. oppos. integr. r. lobat., raro hbae rolub. aphyllx parasit.:

Fam. 9. (116) LAURINEAE Juss., RBr. perig. lobis et stam. plque 4, oppos.; fruct. vario, 1 polysperm.; sem. exalbos. et embr. erectis; - frut. $r$.

flor. arbusc. sempervir., fol. opp., verticill. $v$. alt. integr. $r$. æetiva- incisis: Fam. 6. (113) PROTEACEAE Juss. tione perig. lobis et stam. 3-5, oppos.; fruct. subdrupac. valvat. 1-sperm., sem. pendulo, albos., embr. inverso ; - arb. v. frut. fol. spars. integr. :

† Fam. 1. (108) ANTHOBOLEAE Mart.

stam. penitus 1-adelph. (3-12); flor. dioic.; fruct. 2-vals. 1-sperm., sem. arillato, alb. ruminato ; - arb. fol. alt. petiolat. coriac. integerr.:

? Fam. 10. (11) MYRISTICEAE RBr. 

rhizoparasitæ, aphyllæ, plque stipitibus squamato-bracteatis

Ord. VII. (21) STYLANDRAE Pl. fungoso - carnosie,

flor. capitato-spadicinis 1-sexual.; $\sigma^{\jmath}$ stam. 1-4, filament. plque connat., + fruct. coriac. sicc. $\nabla$. subbaccat. 1-sperm., sem. pendulo :

Fam. 1. (118) BALANOPHOREAE Rich. flor. solitar. $\nabla$. axillar., hermaphr. $\nabla$. abortu 1-sexual.; stam. num. vario, colnmnae centrali insertis; ovar. inf. $\nabla$. semiinf.; fruct. baccat. $\nabla$. subcoriac. intus pulposo, polysperm., sem. parietal.:

Fam. 2. (119) CYTINEA Brogn. e. e. Ldl. *)

fol. apice utriculum operculatum gerentibus; fl. dioic., perig. 4-phyll. ; $\delta$ stam. in columnam connat.; 우 ovar. sup., stigmate sess:; fruct. capsular. 4-loc. 4-valv. polysperm., sem. adscendent.:

†Fam. 3. (120) NEPENTHEA Ldl.

caule herbac. $\nabla$. frutesceate foliato

fol. simpl. plque cordatis ; flor. hermaphr., ovar. inf., perig. 3-part. v. irreg. lingulato; stam. plque pistillo adnat.; stigm. 6 stellatim patentibus; fruct. caps. $\nabla$. bacc. 6-loc. polysperm., sem. placent. centralibus:

Fam. 4. (121) ASARINEA RBr.

\section{Class. VI. THALAMANTHAE.}

Ord. I. (22)

\section{CYATHIN}

Pl. hbae v. rar.

frut.; stam.

et corollae

lobis alter-

nis

stam. et cor.

lobis oppos.;

cor. plque

5-fid. ( raro

5-petal.)

cor. scariosa persist., limb. 4-fido; fruct. 1-v. pleiosperm., pericarp. tenui circumscisso:

Fam. 1. (122) PLANTAGINEAE Juss. cor. irreg. subbilabiata; stam. 4; fruct. 1 -sperm. indehisc.; florib. in receptaculo comm. paleaceo dense aggreg., involucro polyphyllo cinctis :

cor. Fam. 2. (123) GLOBULARINEAE DC. **)

limbo 5- fid. cor. reg. tubo supra orarium contracto, limb. 5-dent v. subintegro; stain. $1-10$, plque 5; fruct. 1-sperm. corollae basi indurata incluso:

Fam. 3. (121) NYCTAGINEAE Juss. ***) styl. v. stigm. 5 (raro 3 マ. 4); fruct. 1-sperm., sem. pendul.; hbae $v$. raro frut.:

Fam. 4. (125) PLUMBAGINFAE Juss., Vent. styl. 1 , stigm. (hbae v. suffrut.; fruct. capsular. polyspermo: simpl. v. lobat.; Fam. 5. (126) PRIMULACFAE Vent. sem. placenta
centrali libera arb. v. frut.; fruct. drup. v. baccat. pleio-v. affixis; embr. abortu 1-spermo:

hilo parallelo (Fam. 6. (127) ARDISIACEA Juss.

-) Wir trennen nach Lisdery dic Nepentlo a e nun von dieser Familie, von der sie in wesentlichen Puncten abweichen.

*) Die Globularineen haben eigentlich eine nảhere Verwandtschaft mit den Dipsaceen als mit den Plantagineen, aber der Anfänger wird sie ihres freien Fruchtkuotens wegen doch hier suchen, von wo sic eben den Uebergang zu den $A$ g gregat ac bilden.

*.) Wenn gleich dic Blüthe der Nyctagineen in manchem Betracht für monochlaundisch zu 


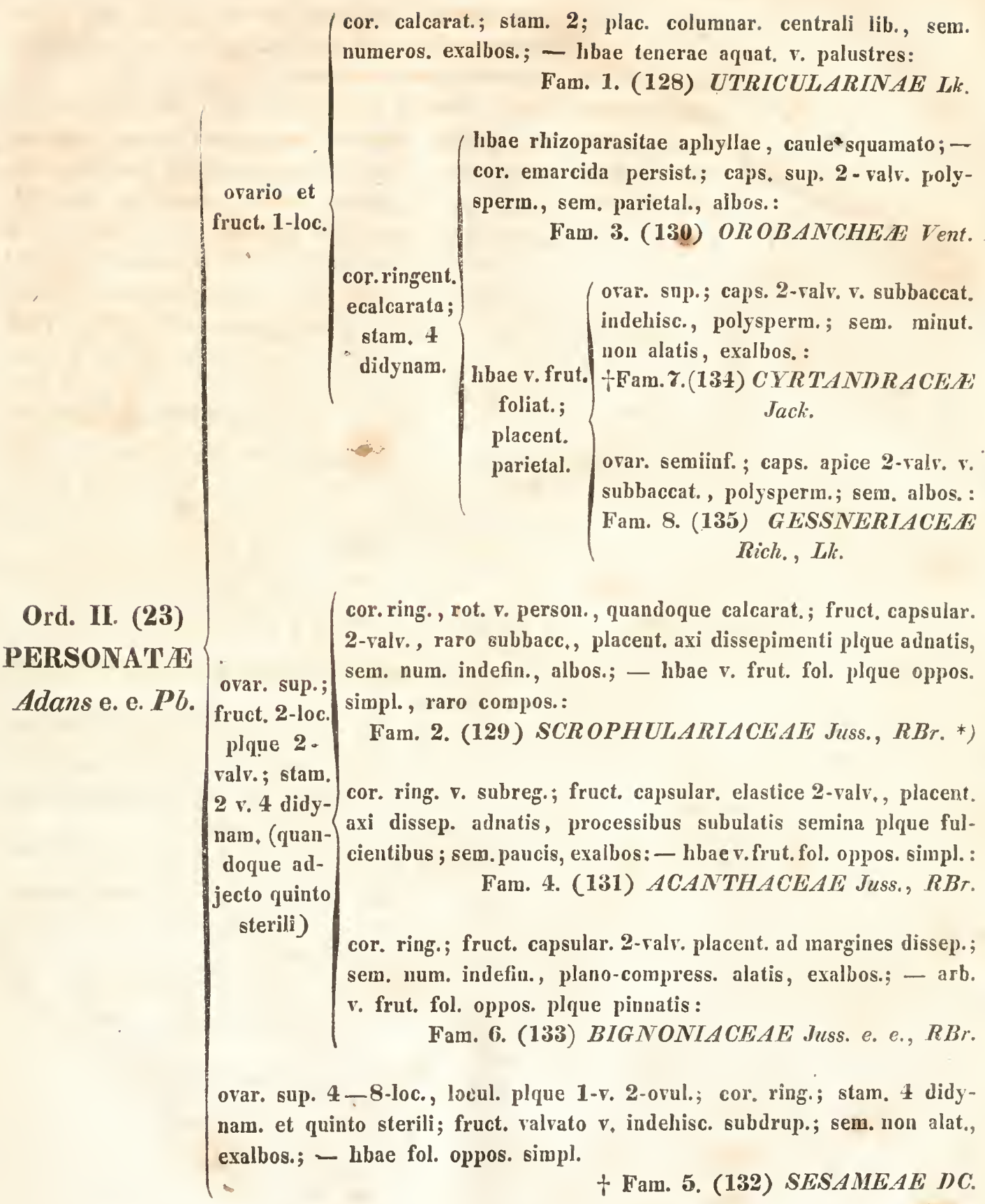

Lalten ist, so seheint dennoch hier die passendste Stelle im Systeme für dicse Familie zu seyn.

-) Die Unterscheidnng der Scrophularina und Rhinanthex (Pediculares Juss.) ist weder in der von Jussizu oder von Decandolus, noch in der ron Lispley angegebenen Umgrenzung haltbar, daher sind sie hier nach $R$. Brown vereinigt. 
cor. regul. fruct. baccat. didymo v. caps. 2-loc., 2 -sperm.; sem. erect. (raro 4-pe- exalbos.; - frut. saepe volub., fol. oppos. pinu., tern. v. simpl.: tal. v. 0); Fam. 1. (136) JASMINEAE Juss. e. e. RBr. staminib, 2, fruct. drupac. v. baccat. v. caps: 2 -loc. $2-4-(v$. abortu 1-) antheris sperm.; sem. pendul. albos.; - arb. $v$, frut. fol. oppos. simpl., 2-locul. raro pinnatis; Fam. 2. (137) OLEINEAE Lk.

Ord. III. (24) PYRENACE $V$ ent. c. add. $P b$.
Ord. IV. (25) TETRACARP $\mathrm{E}$ Pb. (cor. subbilabiata 4-v. 5-fid.; stam. 4, rar. 2; fruct. subdrupac., putam. 2-4loc., loc. $i$-sperm., v. pyreniis $2-4$ staminib. 4 discretis; sem, erect. exalbos.; - arb., didynamis frut. v. raro libae fol, plque oppos. (v.2 v.rariss. simpl. v. comp. : 5, quinto Fam. 3. (138) VERBEN ACEAE Juss. sterili) cor. bilab. v. reg.; stam. 4 v. á, quinto steriti; fruct. drup. putam. 2-4-loc., loc. 1-2-sperm.; sem. pendul. albos.; - frut. fol. alt. v. oppos. simpl.:

†Fam. 4. (139) MYOPORINEE RBT.

cor. plque reg.; stam, 5, corollæ lobis alt.; - fol. plque alt. asperis cor. subbilabiat. irregular.; stam. plque 4 didynam. cor. ring.; stamin. 4 didynam. v. 2 ; caryops. (nucibus) 4 distinct.; hbae v. frut. caule tetragono; fol. oppos., inflor. subverticillata :

Fam. 1. (142) LABIATAE Juss. nucib. 4 distinct. $v$. raro connat.; sem. solitar. exalb., embr. recto; styl. simpl.; - hbae v. raro frut., inflor. plque circinato-racemosa:

Fam. 2. (143) BORRAGINEAE Juss. e. e., Vent. *) cor. subreg. 4-5-fid.; stam. 4 subaequal., raro 5, quinto sterili; ovar. 2 - loc. 2 - orul.; fruct. sicc. indehisc. abortu 1 - sperm.; sem. erect.; - frut. fol. verticill. simpl. coriaceis :

† Fam. 6. (141) STILBINEAE Kth. anther. 1 -loc.; stam. 4 didynam., rar. 2; cor. irreg. 5 -fid.; fruct, sicc. 2-loc., loc. 1-sperm.; sem. erect. albos.; - hbae v. suffrut. fol. alt. v. fascic. simpl.:

\section{† Fam. 5. (140) SELAGINEAE Juss.} (carpell. 5 pluribusve distinct., basi connat.; sem. solitar. albos., embr. curv.; styl, simpl. capitato; - hbae plque prostratae:

fruct. ? T Fam. 3. (145) NOLANACEA Ldl. drupac. drupa e carpellis 4 conuata; sem, solitar. exalbos., embr. saepe curvat.; styl. 2 - r. 4-fido;-arb. v. frut., inflor. plque paniculata :

Fam. 4. SEBESTENEAE Vent. **)

-) Nach Schaders's Vorgange haben v. Martius und Lindery als besondre Familie ron den Bo r ragi-: ne e $n$ ausgeschieden die ( $f$ Fam. 144) HELIOTROPIEE Schrad. ovar. et fruct. unico (e carpell. 4 penitus connatis), stylo terminali.

*) v. Martius theilt diese Familie in zwei, deren eine sich den Borragineen, insbesondre den II el i otropicen, die andre den Convolvulaceen nahe anschliefat, nämlich: 
cor. plque 5 - plicata; sem. embr. curvato

Ord. V. (26) LURID $A$

Limn. c. add. Pb. styl. 1, stigm, integr. v. 2 - lob.; fruct. 2 - (rar. $3-3$-) loc. saepe capsular. 2 - valv. v. operculato, saepius baccat., placent. central. dissep. adnatis; sem. num. indefinito, alb. carnos.; lhbae, frutic. v. arb., quandoque spinos., fol. alternis simpl. . raro pinnatisectis:

Fam. 1. (148) SOLANEAE Juss, e. e. Bartl.

styl. 1-2, stigm. 2, quandoque 2-fid.; caps. 2-4-loc. 2-4valv. v. circumsciss. v. subbaccata indehisc., loc. 2-v. 1 - sperm.; sem. basi affix., alb. parco mucilaginos., embr. cotyled. corrugatis; - hbae v. frut., plque volub., saepe lactesc., fol. alt. simpl. saepe lobat., quandoque pinnatisect., v. 0 :

Fam. 3. CONVOLVULACEAE Juss., Vent. ${ }^{*}$ )

cor. squamulis geminatis ad faucem; styl. 1, stigm. 2-fid.; caps. 1 - tre. 2 - valv.; sem. num. defin. v. indefin., alb. cartilag.; hbae fol. oppos. v. alt. plque pinnatisect., asperis :

corolla non plicata; sem. embr. recto
† Fam. 2. (149) HYDROPHYLLEAE RBr., Schrar. styl, et stigm. 2-3; caps. 2-3-loc. 2-3-valv, v. circumsciss., placent. centralib. $2-3$ dissepim. adnatis; sem. numeros. parv., alb. carnos.; - hbae, quandoque spinos., fol. alt. simpl.:

corolla ad faucem non † Fam. 4. (152) HYDROLEACEAE RBr. squamigera styl. 1, stigm. 3; caps. 3 - locul. 3 -valv., placenta central. triquetra; sem. solitar. v. indefin., albumine carnos.; - libae v. rar. frutices fol. alt. v. oppos., simpl. v. pinnat.:

Fam. 5. POLEMONIACEA Juss., Vent. ${ }^{* *}$ )

SEBESTENEA
Vent. $\left\{\begin{array}{rr}\text { styl. stigm. 2-lobo; sem alb. tenui carnos., embr. cotyled. plano-convexis: } \\ \text { + (Fam. 146) EHRETIACEA Mart. } \\ \text { styl. stigm. dichotomo (4-fid.); sem. e funiculo adscend. elongato pendulis, exalbus., } \\ \text { embr. cotyled. plicatis: }\end{array}\right.$

") Nach Prese, Barteivg n. A. wäre Cuscuta ron den Convolvulace a auszuscheiden:

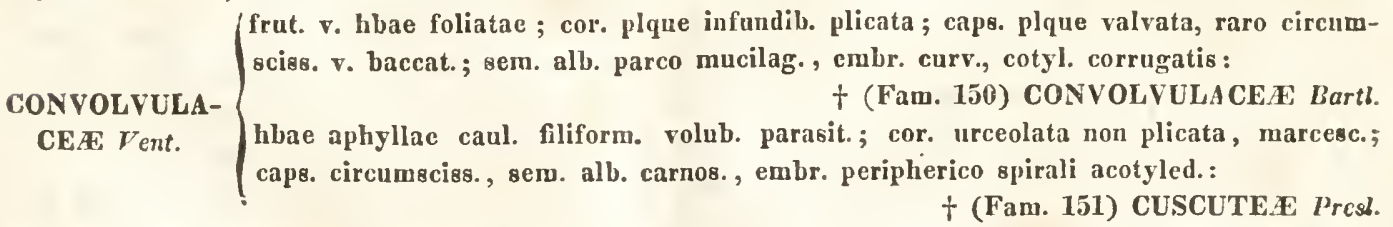

*) Die Gattung Cobæa hat Dos zum Typus einer eigenen Fanilie gemacht, welche eine Mittelbildung zeigt zwischen den übrigen Polemoniaceen und den Bignoniace en:

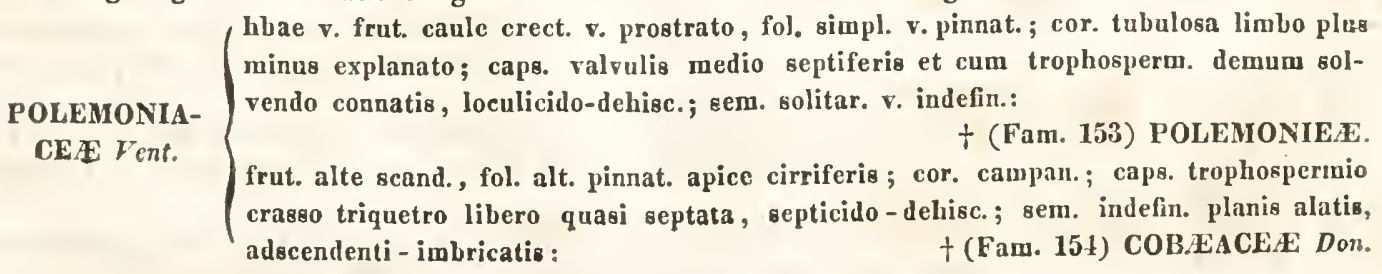


stam. 5, jlque cum stigmate peltato in stylostegium rarie appendiculat. connatis; polline in massulas solidas coalescente; ovar. et styl. 2 , stigm. unico his communi; fruct. follicul. 2 v. abortu 1 , polysperm.; sem. imbricat., saepe comosis; embr. recto; - hbae $r$. frut. saepe volub. et lactesc.:

Fam. 3. (160) ASCLEPIADEAE RBr.

Ord. VI. (27) PICROCHYLEA $P b$. stam. 5 (rarius $4-10$ ); ovar. 1 , quandoque didymo; styl. 1, rar. 2 ; caps. 1-2-loc. plque 2-valv., marginib. inflexis; sem. numeros. parv.; fruct. - hbae, raro frut., non lactesc., fol. oppos. capsular. plque exstipulat.:

$\left(\begin{array}{c}\text { cal. et } \\ \text { cor.lobis } \\ \text { atque } \\ \text { staminib. } \\ \text { num. } \\ \text { spermo }\end{array}\right.$

stam. 5; orar. et styl. 2 v. 1 ; follicul. 2 distinct. $v$. in caps. connat.; sem. saepe comos.; - frut. $\nabla$. arb., raro hbae, sæpe lactesc., fol. oppos. exstip. : Fam. 4. (161) APOCYNEA Juss. e. e. DC.

fruct. succulent. oligosperm. unico $\mathrm{v}$. duplicato, bacc. $\mathrm{v}$. drup.; sem. plque peltatis; - arb. $v$. frut. saepe lactesc., fol. oppos. exstip. :

Fam. 5. STR YCHNEA Pet.-Th., DC. **)

cal. et cor. lob. atque staminib. (1-10) num. plque inæqual.; ovar., styl. et stigm. 1.; fruct. capsular. v. baccat. polysperm. v. drupac. 2-4-pyreno; sem. erect. v. peltatis;-arb. v. frut., raro hbae, non lactesc., ramis nodoso-artic., fol. oppos., stip. interpetiolarib. plque vaginantibus :

† Fam. 2. LOGANIACEAE Bartl., Pb.***)

") v. Martics theilt dicse Fanilie in drei folgendermafsen zu unterscheidende:

(fol. (plque) alt., . sacpe dentat. v. compos., petiolis basi dilatatis; cor. barbata, lobis aestivat. inflexis; styl. continuo, stigm. bilobo; fruct. valvulis axe scminiferis:

GENTIANEE

Juss.

f (Fam. 155) MENYANTHEE Mart.

(fol. exstipul. ; cor. aestivat. contorta, saepe marcesc.; styl. contin., stigm. 1 v. 2 ; fruct. valvular. marginib. placentiferis :

fol. oppos.

+ (Fam. 156) GeNTIANEA Juss. e. e. Mart. fol. substipulat.; cor. aestivat. valvata; styl. articulatim inserto, stigm. simpl.; fruct. subdicocco, placent. central. lib.:

+ (Fan. 158) SPIGELIACEF Mart.

*) Mehre neuere Botaniker vereinigen nach dem Vorgange Brows's die Strychneen wieder mit den Apocyneen; ron Andern sind dagegen noch weitere Trennungen vorgenommen worden, närnlich :

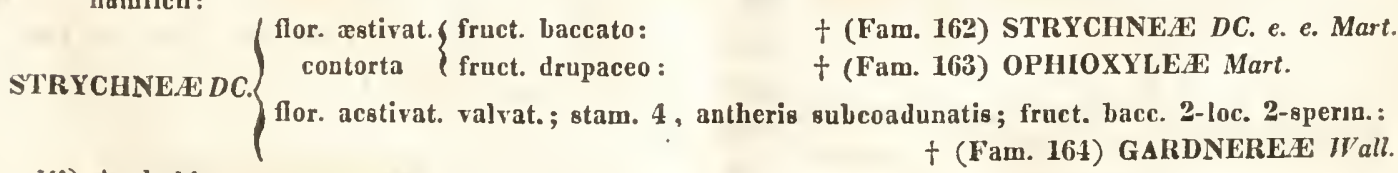

*") Auch hier tremnen $r$. Martius, Lindegx etc. zwei Familien:

LOGANIACEA $\left\{\begin{array}{r}\text { staw. } 5 \text { v. 1; fruct. 2-loc. capsular. polysperm. v. drupac. pyrenis 1-2-spermis: } \\ +(\text { Fam. 158) LOGANIEAE RBr., Mart. }\end{array}\right.$

Nob. $\quad$ stam. $10-5$; fruct. 2-4-loc. Waccat. polyspermo :

t (Fam. 159) POTALIEA Mart. 
Ord.VIII. (28) ATAX $P$ C . orar. loc.

1-2- (rar. 4-) ovulat.; fruct. drup. v. baccat. pluriloc.

antheris

2-loc.

ovar. sup. 


\section{Class. VII. CALYCANTHE.}

fol. rerticillat.; stip. 0 ; achaen. didymis, loc. 1 -sperm.; - hbae v. suffrut. caule plque tetragono:

Fam. 1. (1\%ఖ) STELLATAE Linn. e. e. DC.

Ord. I. (29) RIGIDAE

Batsch. c. ald. Pb.
fruct. capsular. v. baccat. 2-v. pluri- loc., loc. 1-sperm; - frut. v. arb., raro hbae, stip. saepe raginantibus:
Fam. 2. (185) COFFEACEAK DC. c. $a d d$. $P b$.
fruct. capsular., baccat. v. drupac. 2 - v. pluriloc., loc. polysperm.; - frut. v. arb., raro libae:
Fam. 3. (176) CINCHONACEAK DC. Théor. él.

florib.»receptac. comm. insidentibus, plque involucratis; fruct. capsular. v. baccat. 2-v. pluriloc., loc. 1-v. pleiosperm.; - frut., rarius arb.:

Fam. 4. (178) CEPHALANTHEAE Juss., Pb.

fol. v. integerrim. extipulat, v. rarius serratis $\mathbf{v}$. lobat. v. pinnatis; fruct. baccat. 1-4-loc., loc. 1-v. pleiosperm.; - frut. v. arbusc., rariss, hbae.:

Fam. 5. CAPRIFOLIACEAE Juss. e. e., A. Rich. *)

") Schon Batsca und neuerlich Barthing, v. Martus u. A. theilen diese Familie in zwei, nämlich:

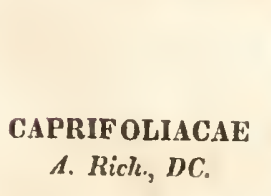

A. Rich., DC. fol. integris extipulat.; cor. tubulosa, saepe irreg.; stylo fliformi, stigm. indiviso v. 2-3-fido:

† (Fam. 178) LONICEREAE RBr. (CAPRIFOLIACEAE Bartl.)

fol. saepe serratis, lobat. $r$. pinnat., plque stipulat.; cor. rotata v. campanul., reg., styl. 0, stigm. 3 sessil. 


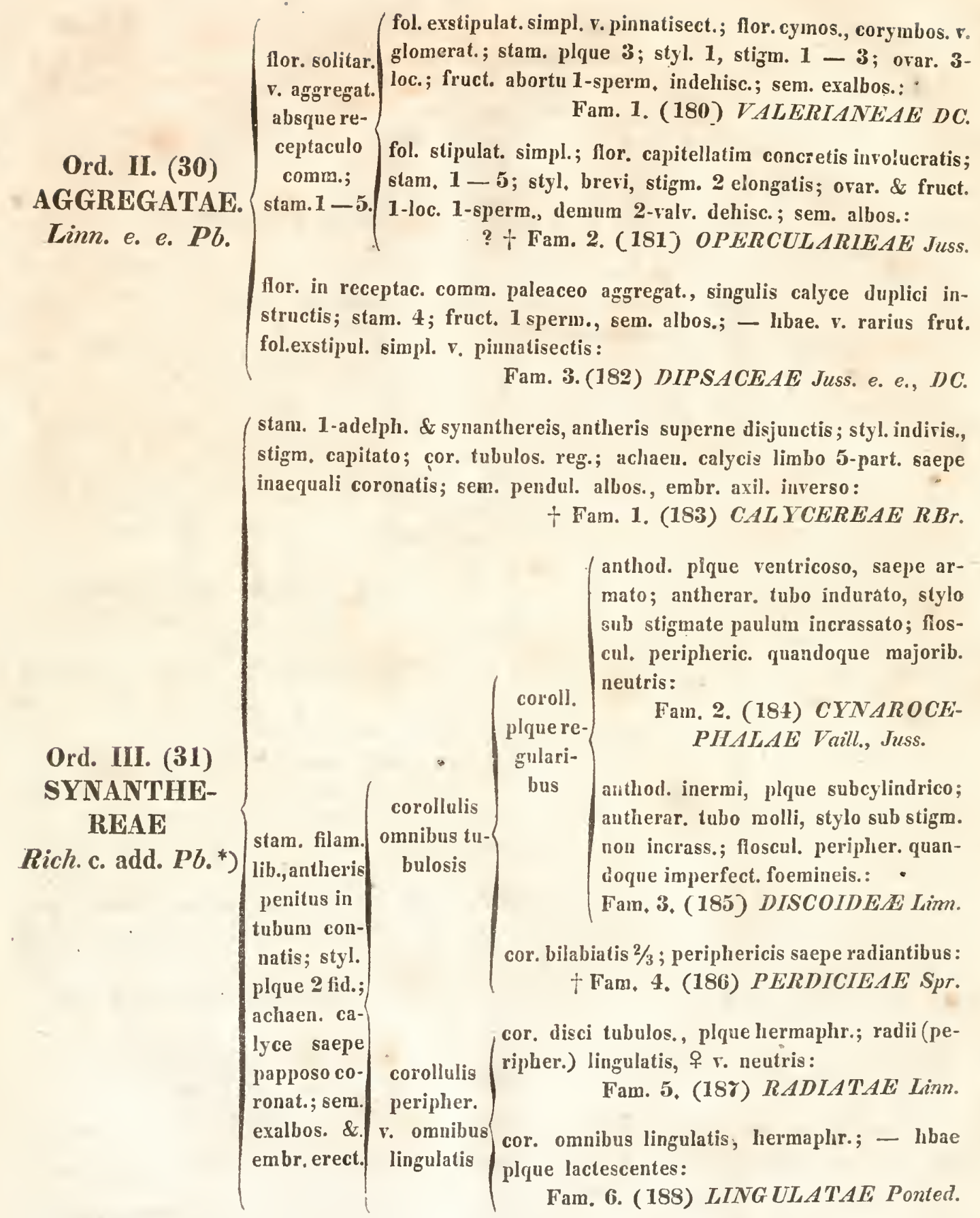

") Die neueste Eintheilung dieser Ordnung (der GOMPOSITAE Vaill., Linn., DC. \& c. mit Ausschlufs der Calycereen) hat nach den grofsen Vorarbeiten von H. Cassisi und Chr. Fr. Lessixg jüngst Decandole in seinem Prodrom. Vol. 5. (1836) gegeben; wir fügen auch davon hier eine diognostische Tabello bei: 


\begin{abstract}
(stam. cum stylo connatis; antheris 2, plque didymis; hbae v. suffrut, non lactesc. :

Fam. 1. (189) STYLIDIEAE RBr.

\section{Ord. IV. (32) RAPUNCULEA} Pb.

cor. irreg.

stam. 5, a stylo liberis, antheris saepe con- styl. stigrmate (bilobo $v$, integro) ciliato; cor. natis.

styl. stigmate (integro v. bilobo) indusio cyathi formi cincto; cor. plque dorso profunde fissa, aestivat.induplicata; - hbae v. frut., non lactesc.: † Fam. 2. GOODENOVIEAE RBr. ${ }^{*}$ ) plque antice fissa, aestivat. ralvata; - hbae $v$. suffrut, saepe lactesc.:

Fam. 3. (193) I, OBELIACEAE Juss, e. e. RBr.

cor. regul., aestivat. valvata; stam.5, plque liberis; styl. stigmate plque lobato, non ciliato nec indusiato; - hbae v. frut., saepe lactescentes: Fam. 4. (194) CAMPANULACEAL Juss. e. e. Spr.
\end{abstract}

Tululiflor : flosc. hierınaphr. tubulos. regulariter 5₹. rarius $4-$ dentatis.

stylo ;flosculor. bermaphrod. cylindraceo

$$
8
$$
styli ramis apice penicillatis, nunc truncatis, nunc ultra peniciilum in conum hispidulym productis:

Trib. 4. SENECIONIDEAE Lessg.

stylo flosc. hermaphrod. superne nodoso-incrassato \& saepe ad nodum penicillato; stigmatis seriebus non prominulis, apice ramorum conflucntibus:

Trib. 5. CYNAREAE Spr., Lessg.

La bi it i- stylo flosc. hermaphr: superne cylindr. vo subnodoso, ramis plque truncatis flora : flosc. hermaplir. plque bilabiatis extus convexis \& aequaliter puberulis $v$. nudis:

Trib. 6. MUTISIACEAE Lessg.

stylo flosc. hermaphr. nunqnam nodoso-inerassato, ramis linearibus longiusculis truncatis, apice penicillatis:

Trib. \%. NASSAUVIACEAE Cass.

Liguliflorac: flosc. omnibus hermaplurod. ligulatis, stylo superne cylindr. ramis longiusculis aequaliter hirtellis: .

Trib. 8. CICHORACEAE Vaill., Juss.

") Lisduer, fúvtn u. A. trennen von den eigenilichen Goodenovieen noch zwei kleine schon von R. Brqtr angegebene Familien: 
/ stam. 5, plque triadelph., anther. flexuos.; ovar. inf,, stylo brevi, stigm, 3 -5 crass.; fruct. plque succul. polysperm., sem. parietal., exalbos.; - hbae sarmentaceae scand. cirrhis. unilateraliter stipularibus:

flor. 1 - sex.

Fam. 1. (195) CUCURBITACEAE Juss,, Bartl.

stam. 10, anther. subsessil.; ovar. sup., stigm. ã - lob. sess.; fruct. succul. polysperm., sem. parietal. albos.; - arb. non ramosae, lactesc, fol, subterminal., louge petiolat., palmatis:

\section{Ord. V. (33)} PEPONIFERAE Pb.
† Fam. 2. (196.) PAPAYACEAE Mart.

(cor. saepe duplicata, interiore plque filamentosa; stam. 5 mouadelpl.; ; orar. supra stam. exserto, stigm. 3 clavatis; fruct, plque succul. polysperm., sem. parietal. albos.; - hbae. v. frut. (rariss.arb.) plque scand., cirrhis axillar., fol. alt., stipulat.:

Fam. 3. (197) PASSIFLOREAE Juss. e.e. ovar.sup.

stipitato cor. sub-5-petala, coronula interiore brevi; stam. 5 v. 10 liberis v. stipiti orarii adnatis; ovar. stylis 3 longis filiformib., stigm. clavatis; fruct. caps. apice 3 valv. polysperm., sem. adscendent. albos.; - hbae suffrut. non scand. nec cirrhos., fol. exstip.:

? † Fam. 4. (198) MALESHERBIACEAE Don.

ovar. inf.; cor. saepe duplicata, radiatim plicata; stam. 5 submonadelph. v. indefin. lib.; styl. 1, stigm. lobat.; fruct. succul. pleiosperm.; — frut. fol. alt. exstip.

† Fam. 5. (199) BELVISIACEA RBr.

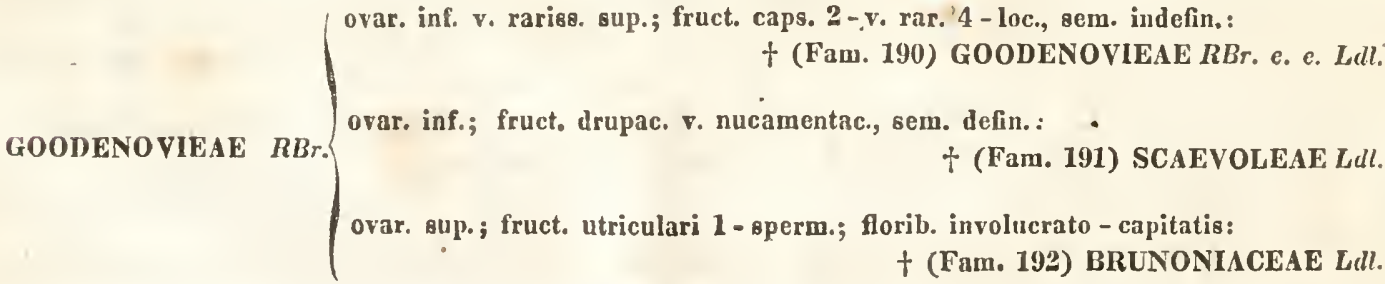




\section{Class. VIII. CAL YCOPETALAE.}

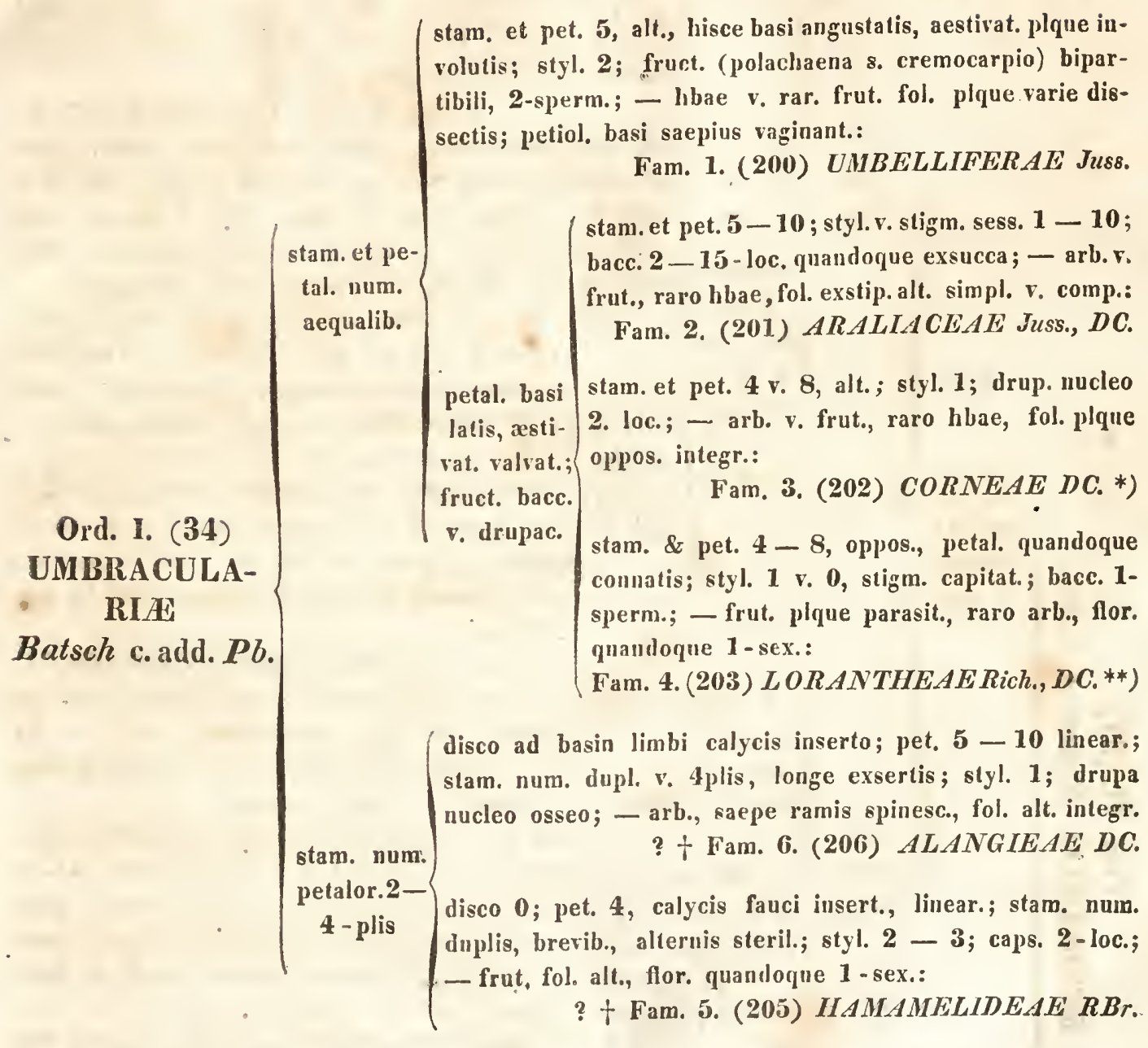

-) Dreaspoler hat mit Recht die Gattung Hedera aus dicser Familie zu den Araliace en versetzt, daher denn der frühere Name der Fam. "H ederacca e" geändert werden musste. Ich zähle übrigens mit Bardung auch die Gattung Marlea hieher.

*) Die († Fam. 204) RHIzophoreaE RBr., welche Jussieu mit den L o r a nt he en verband, bilden cine wohlunterschiedene Gruppe (arb. v. frut., fol. simpl. oppos., stip. interpetiol.; cal, limb. 4-13 lob., petal. totidem calyci infert.; stam. petal. num. 2- v. 3-plis; ovar. inf. 2-loc., locul. 2-v. multiovulat, styl. 1; fruct. indehisc. abortu 1-sperm., sem. pendul. exalbos., saepe e fructibus germinante); nach ihren Cliarakteren sind sie in dic Nähe der Memecyleen, Combretaceen u. Cunouiaceon zu stellen. 
cal. 3-part, pet. et stam. 3, altern., imo calyci insert.; ovar. sup., styl. sub 0 , stigm. plur.; bacc. $3-6-10 c$. 3-9-sperm., sem. erect. albos.; - fruticuli sempervirentes fol. et flor. parvis:

ol. ex-

† Fam. 1. (207) EMPETREAE Nutt. stipulat. cal. 5-part.; pet. et stam. 5, alt.; ovar. semiinf., styl. 2 v. 1 ; fruct sicc. indehisc. 2 - v. 1 -loc. v. 2 -cocc., locul. 1 - 2 -sperm., sem. pendul. albos.; - frut. fol. parv. rigidis confertis, flor. parv. aggregat.

+ Fam. 2.(208) BRUNIACEAE RBr.

fol.

simpl.

fl. hermaphr.v.

abortu

1 -sex. fol. fol.plque stipulat.; cal. 5 - $\mathbf{v} .4-$ part.;pet. \& stam. 5 V. 4 cal. aestivat. ralvat.; pet. \& stam. oppos.; ovar. plque cal. adnat.; styl. 2 - 4-part.; fruct. drupac., baccat. v. caps. 1 -4-loc. (sæpe 3-cocc.), loc. 1-sperm., sem. erect., non arillat., subalbos.; - frut. v. arbusc., ram. saepe spinesc., fol. plque alt., sa epe stipulat., flor. parv.:

Eam. 3. (209) RHAMNEAE Juss. e. e. DC. stigm. 2-4-fici; fruct. caps. 2 - 5 - loc. 2 5 -valv., v. rarius drupac. v. samaroid., locul. cal. xsti- $1-\nabla$. oligospern.; sem. erect. arillat. albos.; vat. im- frut.v. arb. fol. plque alt, stip. decid.:

bricata; Fam.4. (210) CELASTREAERBr. e.e.Pb.*) pet.et pet. plque 2-lobis parv.; styl.1 - 3; drup. stam.alt.; coriac. putamine 2-3-loc., loc. 1-sperm., ovar.sup. sem. pendul, exalbos; - arb. v. frut. fol. alt. stipulat.:

? +Fam.6.(212) CHAILLETIACEARBr.DC. (fol, oppos. pinnat. v. tern, stipul. decid.; - cal. 5-part.; pet. et stam. 5 alt., disco insert, ovar. sup. substipitato; styl. 2 - 3; fruct. inflato-capsular. v. haceat. 2 - 3-loc., loc. 2-3-sperm., sem. horizontalib., alb. sub0; - frut. $\checkmark$. arbuse.:

plque fol. alt. exstip., saepius pinnat,; - cal. 3 -5 - part.; pet. $3-5$ compos. v. 0; stam. v. num. pet. aequal. et iisdem alt., v. 2-v. raro 4-plis; ovar. plque sup. $1-6$, quibusdam saepe abortientib.; fruct. caps. v. drup. oligosperm., sem. pend. v. erect. exalbos.; - arb. v, frut. cortice plque balsamifero:

Fain. 7. TEREBINTHACEAE Juss., DC.e.e. Pb.**) flor. 1 - oic. dissimili structura: $\delta$ amentac. pet. 0 , stam. $3-6$; $q$ subsolitar. pet. $4 \nabla$. 0; ovar. inf., styl. $1-2$, stigm. lobatis; fruct drupac. 1 -sperm., sem. erect. albos.; - arb. fol. pinn.:

Fam. 8. (220) JUGLANDEAE DC.

*) Wir trennen hier mit Bartung die Staphile acea e ron R. Brown's u. Decandohe's Celastrinen; auch die AQUIFOLIACEAE DC., Bartl. (ILICINEAE Brongn.) wären wohl wegen ihrer völlig hypogynischen, vielleicht eigentlich monopetalischen Blume auszuscheiden; jedoch ist ihre passendere Stelle im Systeme zweiielhaft.

*) Hicr sind offenbar noch mehre Familientypen zusammengeworfen, allein mit Ausnahme der PTELEACEAE Kth., welche unstreitig zuden $\mathbf{R}$ u taria in die folgende Klasse gehören, sind die Merk- 


\section{Ord. III. (36) LEGUMINOSAE Adans., Juss.}

(cor. papilionacea, raro defectiva; stam. 10, plque $\mathbf{2}$-adelph. $(9+1)$; sem. embr. plque curvato:

Fam. 1. (221) PAPILIONACEA Linn., RBr.

flor. irreg. plque hermaphr.

cor. irreg. non papilionacea, quandoque defectiva $\nabla .0$; stam. num. var., lib., plque inaequalibus, saepe quibusdam steril.; fructu saepius lomentac., quandoque drupac.; sem. embr. recto:

Fam. 2. (222) CAESALPINLACEAE RBr., DC.e.e.Pb.*)

flor. regul., saepe polygam,; cor. 4 - 5-pet., plque hypogyna, petal. quandoque connatis; stam. 4, 5, 10 - 200, monadelph. v. lib.; sem: embr. recto:

Fam, 3. (223) MIMOSEAE RBr.

male nnd Abgrenzungen noch zu unbestimmt, um sie definitiv festsetzen zu könneu. Folgendem sind übrigens die von mehren Botanikern schon getrennten Familien dieser Gruppe:

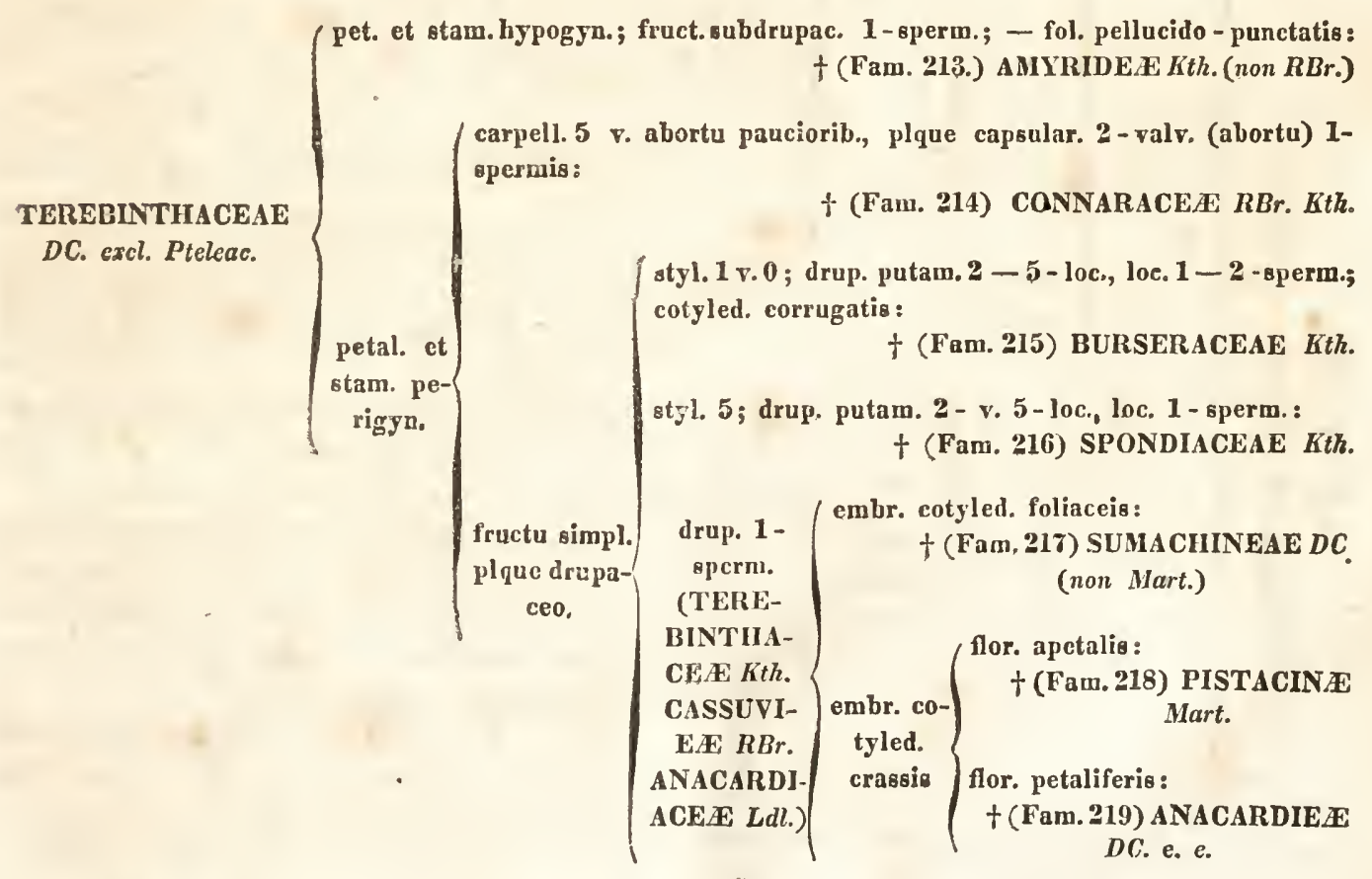

") Die ochmetterlingsblūthigen GEOFFREA $D C$. glaube ich zur ersten Fam., die SWARTZIEA $D C$. hingegen, ungeachtet ihrer widerstreitenden Keimbildung, hielıcr stellen zu dürfen. Hingegen mu「s die zuerst von $\mathbf{R}$. Brown angedeutete kleine Familie ( $f$ Fam. 224) MORINGE $\mathbb{E}$ RBr., Bartl, welche durch eine dreiklappige, die Samen auf der Mitte der Klappen tragende Kapselfrucht selır von den Leguminos en abweicht, ohne Zweifel ausgeschieden werden; wohin sic jedoch im Systeme einzureihen sei, wage ich noch nicht $z$ bestimmen. 


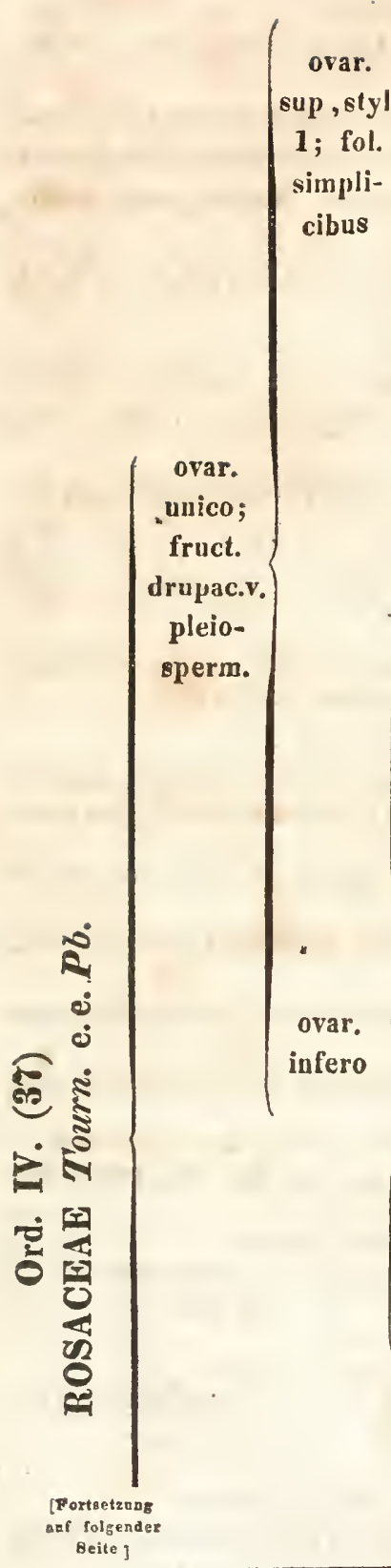

cal. 5. - fid. decid.; stam. defin. $\nabla$. indefin.; fruct. drupac. $1-2$ sperm.; - arb. v. frut. fol. alt. stipulat.:

Fam. 1. DRUP $A C E A E D C$., Spr. *).

cal. tubulos. 4 - 12 -dent. persist.; stam. defin., antlıer. subrotund.

non appendiculatis; fruct. caps. 1 -v. pluriloc. polysperm.; - hbæ

v. rarins frut. fol. plque oppos., exstip.:

Fam. 11. (237) SALICARIACEAE Juss.

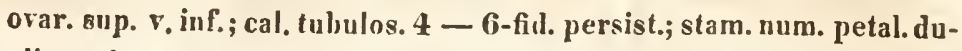
plis, anther, oblong. rostratis, varje appenliculatis; fruct. bacc. v. caps. (pluriloc. polysperm.; - hbae, frut. v. arb. fol. oppos.:

Fam. 10. (236) MELASTOMACEAE Juss., Don.

cal. limbo $4-5$-Iobo persist.; stam. 8 v. 10, anther. incurvis; bacc. 2-4-loc. oligosperm., sem. cotyled, convolut.; - frut. fol oppos. simpl. exstip.:

† Fam. 9. (235) MEMECYLEAE DC.

stam. defin.; styl.

cal. limbo 2-5-, saepius 4-lobo; stam. totidem v. luoc num. dupl., anther. orat. v. oblong.; fruct. caps. v. bacc.

1

2 - 4-loc., plque polysperm.; sem. cotyled. parvis; hbae v. frut. fol. oppos. v. alt. exstip.

Faı. 12. (238) ONAGRARIAE Juss.

cal. limbo 4-5-lobo decid.; pet. 4, 5 v. 0 ; stam. num. lobor. cal. duplis v, raro triplis v. aqual.; drup. baccata nucament., abortu 1-sperm.; sem. colyled. convolut. v. complicatis; — arb. v. frut. fol. simpl. exstip. † Fam. 8. (234) COMBRETACEA RBT.

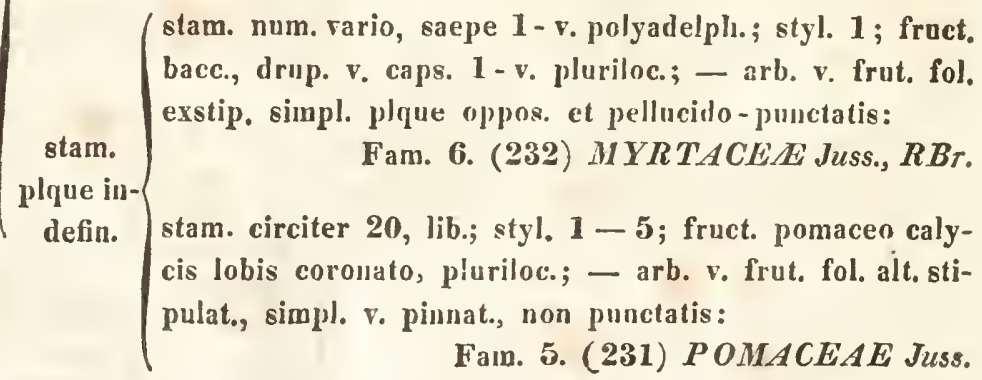

2) Als zwei Familien oder auch blofse Zünfte werden hier noch unterschieden:

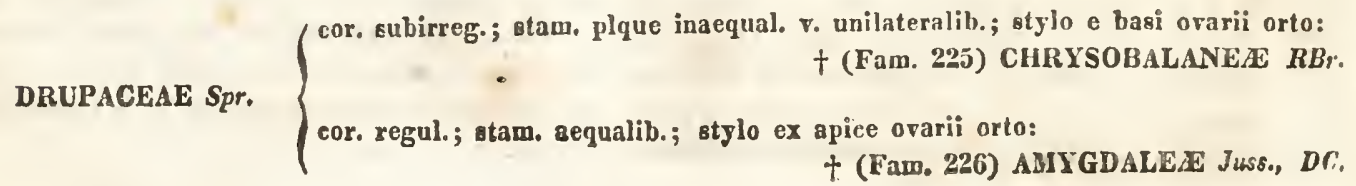



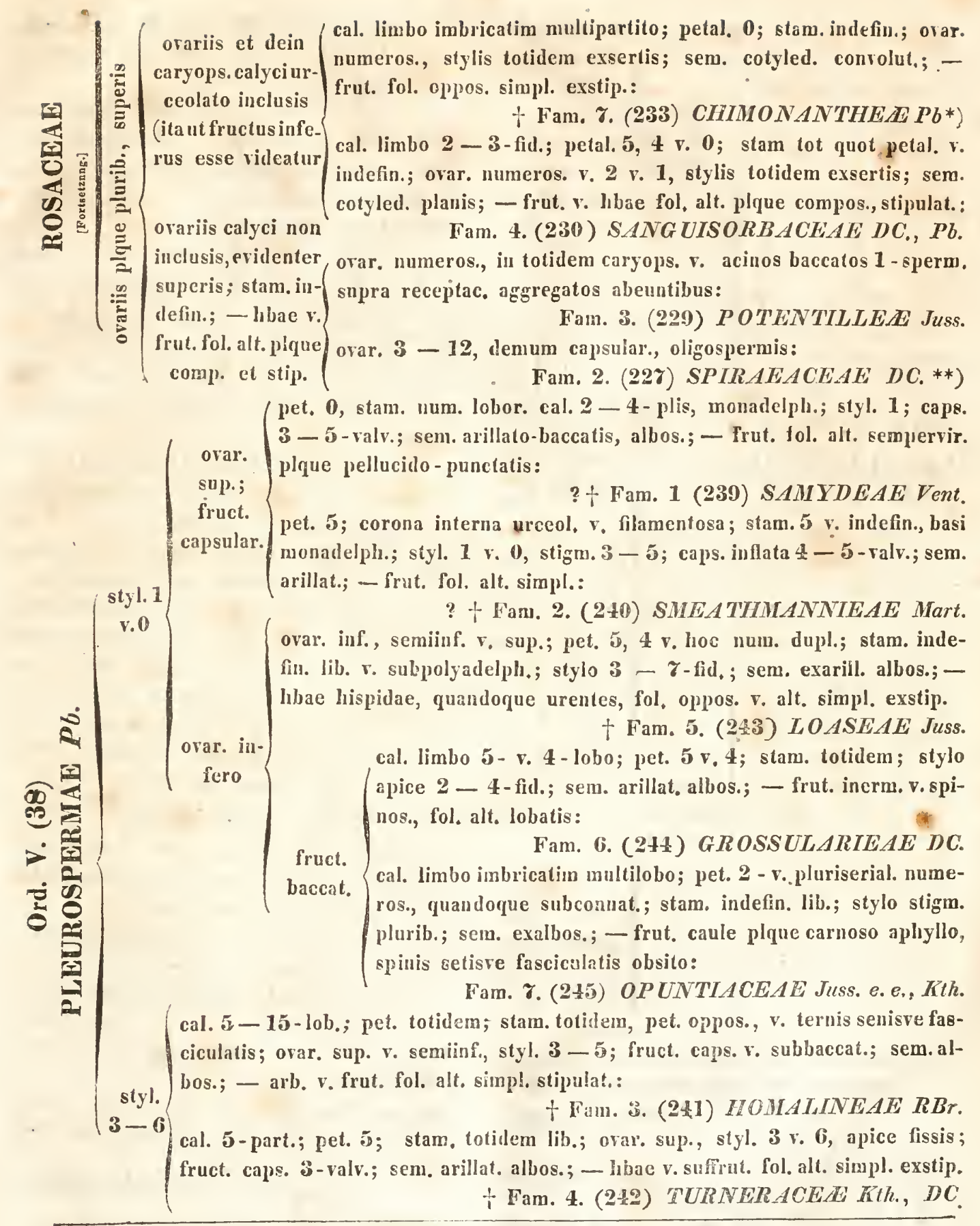

") Diese Benennung lex Fan. subatituire ich der gewöhnlichern „CALYCaNTHEAE" Lindl. wegen der Ähnlichkeit iler letztern mit dem Namen meiner VII. Pflanzenklasse.

") Nach den Charakteren wïrden auch die († Fam. 228) NEURADEE DC. (hbae enffrut. fol. pinnatifid. stipulat.; cor. 5-pet.; stam. 10; ovar. semiinf.; styl. 10; fruct. capsular. e carpellis 10, monospermie; sem. cxalbos, embr. curv.) hier sich anschliefseu; ihr naturgewäfserer Platz dürfte aber neben den Fic oideen sein. 


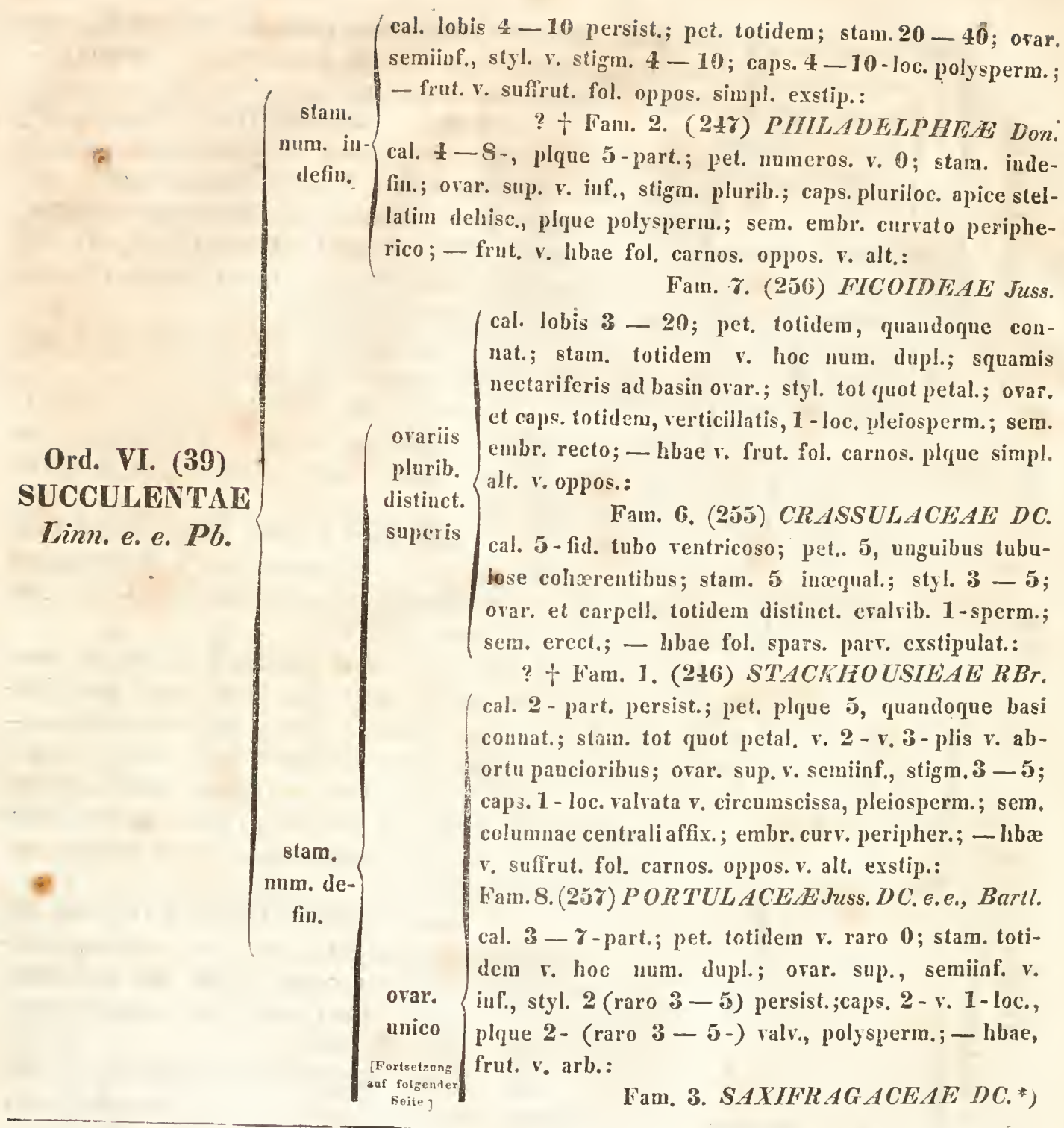

-) Folgende kleine Gruppen, welche Decundole als blofse Tribus der Saxifragace ansieht, werden ron mehren Systematikern als besondere Familien aufgestellt:

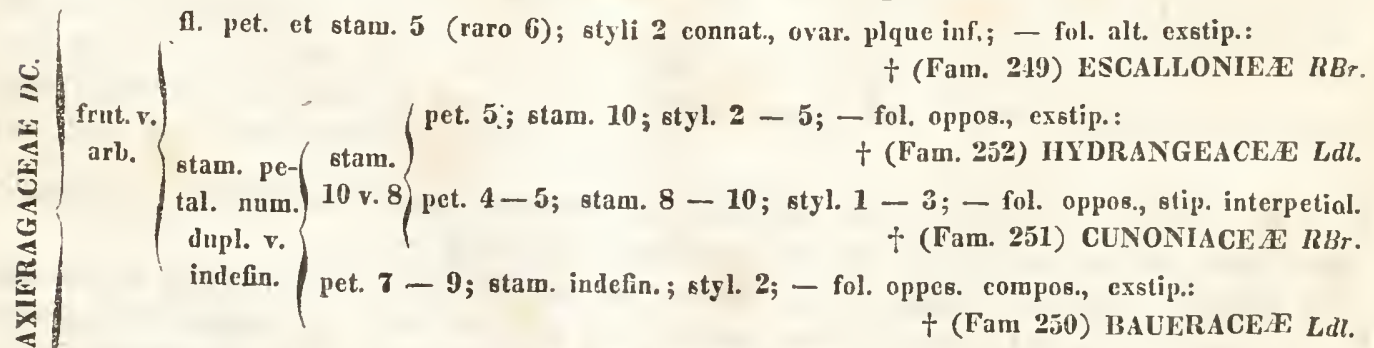

herbaceae fol. exstip. alt. v. raro oppos.; stam. plque petal. num. dupl., quandoque hypogynis:

+ (Fam. 248) SAXIFRAGEAE $D C$. 


\section{SUCCULENTAE}

[Fortgetzutg] stam. num.
defiu.; ovar.
unico

ovar. inf., stigm. $2-4$ sess.; cal. limb. $4-v, 3-$ part. v. sub0; pet. tot quot lob. cal. v. 0; stam. inpl. v. totidem v. pauciorib.; fruct. pluriloc. v. abortu 1 - loc. indehisc., loc. 1 - sperun.; sem. embr. central. recto; - suffrut. v. hbae saepe aquat., fol. alt, v. oppos. :

Fam. 4. (253) H.ALORAGEAE RBr.,Bartl.*)

styl. 1 v. 0 ;

(raro $2-5$ deciduis)

cal. 4 - v. 6 - part. persist.; pet. 4 v. 6; stam. petal, num. 2 -v. 4-plis, alternis steril., lib. v. 1 -adelph; stigm. sess. 3-4.lob.; caps. 3-4-loc. 3-4 - valv. polysperm.; - hbac fol. radical., simpl. v. lyratis:

? f Fam. 5. (25士) GALACINEL Don.

ovar. cal. plque 5 - fid. persist.; pet. 5 v. sup. 0 ; stam. tot quot lob. cal. hisque oppos. v. pauciorib. v. duplis; styl. 2 5 , saepe basi connat fruct. utric. $\mathbf{v}$. capsular. 1 - v. 3-loc., sem. numeros. columnae centraffixis, r. solitar, subpendul.; embr. curv. peripher.; libae v. suffrut. fol. plque oppos., saepe stipulat.; flor. parvis:

Fam. 9. PARONYCHIEAE St. $H_{\imath l}$, Juss., $D C_{. * *}^{*}$.

-) Hicher gehōren eigentlich auch die schon oben S. 11 anfgeführten Familien († Faın. 34 und 35) der IllipURIDEAE $L k$. und CALLITRICHINEAE $L k$, und es schliefsen sich die († Fam. 33. ibid.) CERATOPHYLLEAE Gray zunächst an.

*) Manche Autoren haben diese Fannilie in mehre zerlegt, vorzüglich aber in folgcnde zwei:

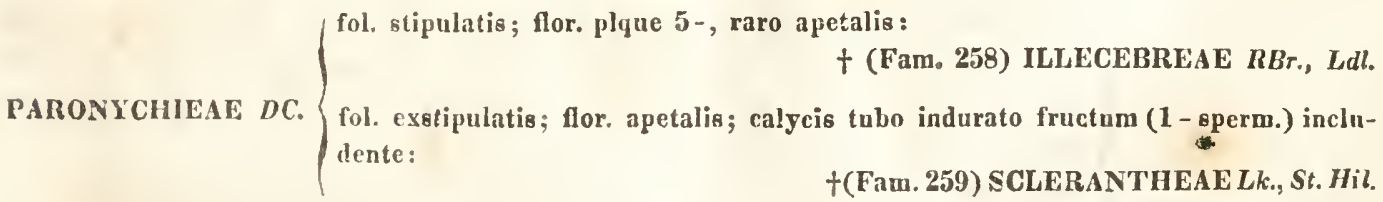




\section{Class. IX. THALAMOPETALAE.}

cal. 5- (ra-, ovar. et caps. 3-v. 5-loc., styl. totidem persist., stigm. capitatis; sem. 3-) sepalo cylindric. exalbos, embr, recto; - hbae decumbentes, flor. axillaribus: v.-part.; pe? + Fam. 1. (260) ELATINEAE Cambess. tal. totidem

exunguicn- ovar. et caps. 1-loc., styl v. stigm. 2 -5 linearib.; sem. reniformibus latis, rar.0;
stam. 3- angulatis, albos., embr. periplierico plque curvato; - hbae v. suffrut., 10; styl. v. flor. plque cymosis:

stigm. $2-5$

Fam. 2. (261) ALSINEAE DC. e.e. Barll. cal. tubuloso 5. dentato; pet. plque longe unguiculatis; stam. plque 10; styl. . stirm. elongat. 2, 3 v. 5; caps. 1 - 5- loc., raro circumsciss. v. subbaccata; sem. albos., embr., peripher. curv.; - hbae, suffrut. v. raro frut.:

Fam. 3. (262) SLLENEAE DC.

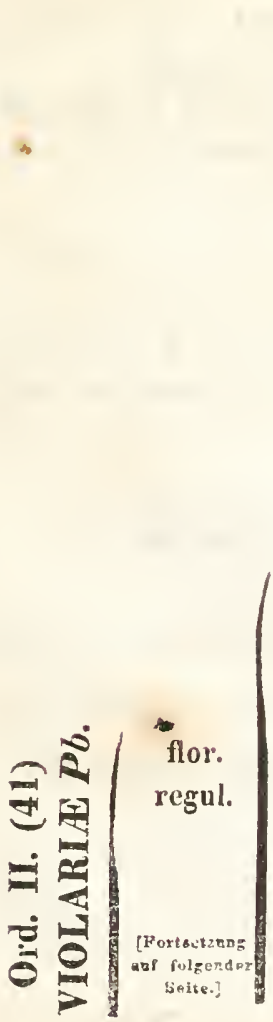

semin. albos., non comosis

(cal. basi tubulos; pet. 5 v. 4 unguicalat.; stam. totidem v. $1-2$ superadditis; stylo 2-3-fid.; caps. 2-4-valv., marginib. valrular. seminifera; - hbae r. suffrut. ramosiss., fol. exstipul. simpl. plque oppos. basi amplexicaul.:

Fam. 1. (263) FRANKENTACEAE St. Hil. petal. et stam. 5 oppos.; corona filamentosa intra corollam; stylo stigm. simpl.; caps. marg. valvular. seminifera; - hbae r. fruticulifol. alt. simpl. stipulat.:

† Fam. 5. (267) SAUVAGESIACEAL DC., Mart.

stam. 5 petal. alteru., v. 10, 15, 20; styl. 3 - 5, rar. 1 ; caps. 3 - 5-valv, basi $r$. mediis ralv. seminifera; - hbae fol. alt. simpl., vernatione saepe circinatis; stip. sub0: Fam. 6. (268) DROSERACEAE DC.

cal. 5- v. 4-part.; stam. tot quot pet. v. hoc nam. dupl., saepe $\mathbf{l}$-adelph.; stylo brevi, stigm. 3; caps. basi v. mediis valv. seminifera; sem. apice comosis, exalbos.; - frut. v. suffrut. ramis virgatis, fol. alt. parvis squamaeformibus :

† Fam. 7. (269) TAMARISCINEAE Desv. caps. pilque 3- valv. poJysperm; petal. tubulose connatis; stam. $10-12$ lib. exsertis; stylo 3 sem. embr. fid.; caps. mediis ralv. seminifera; sen. marg. alatis, albos.; recto ) frut. v. arlo. ramis spinos., fol. simpl. spars.:

+ Fam. 8. (280) FOUQUIERACEAE DC, Fth. 


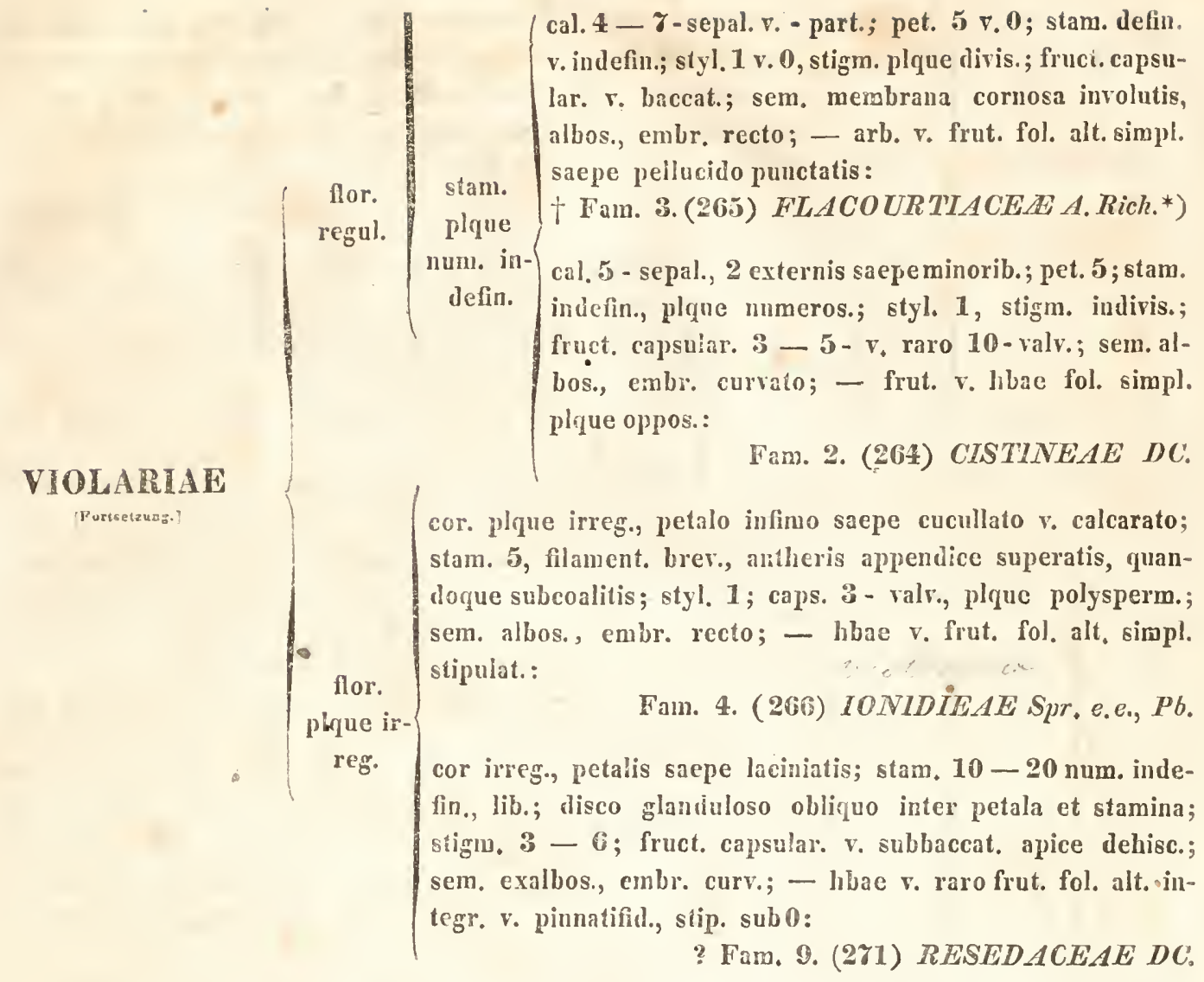

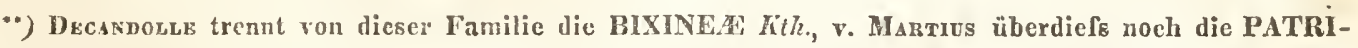
SIACE Ricusnd halten mach den Ergebnisse spütercr Untersuchungen diese Gruppen für Bestandtheile einer einzigen Familic, wclcher Ansicht wir deun vorlänfig hier folgen. 


\section{- 38}

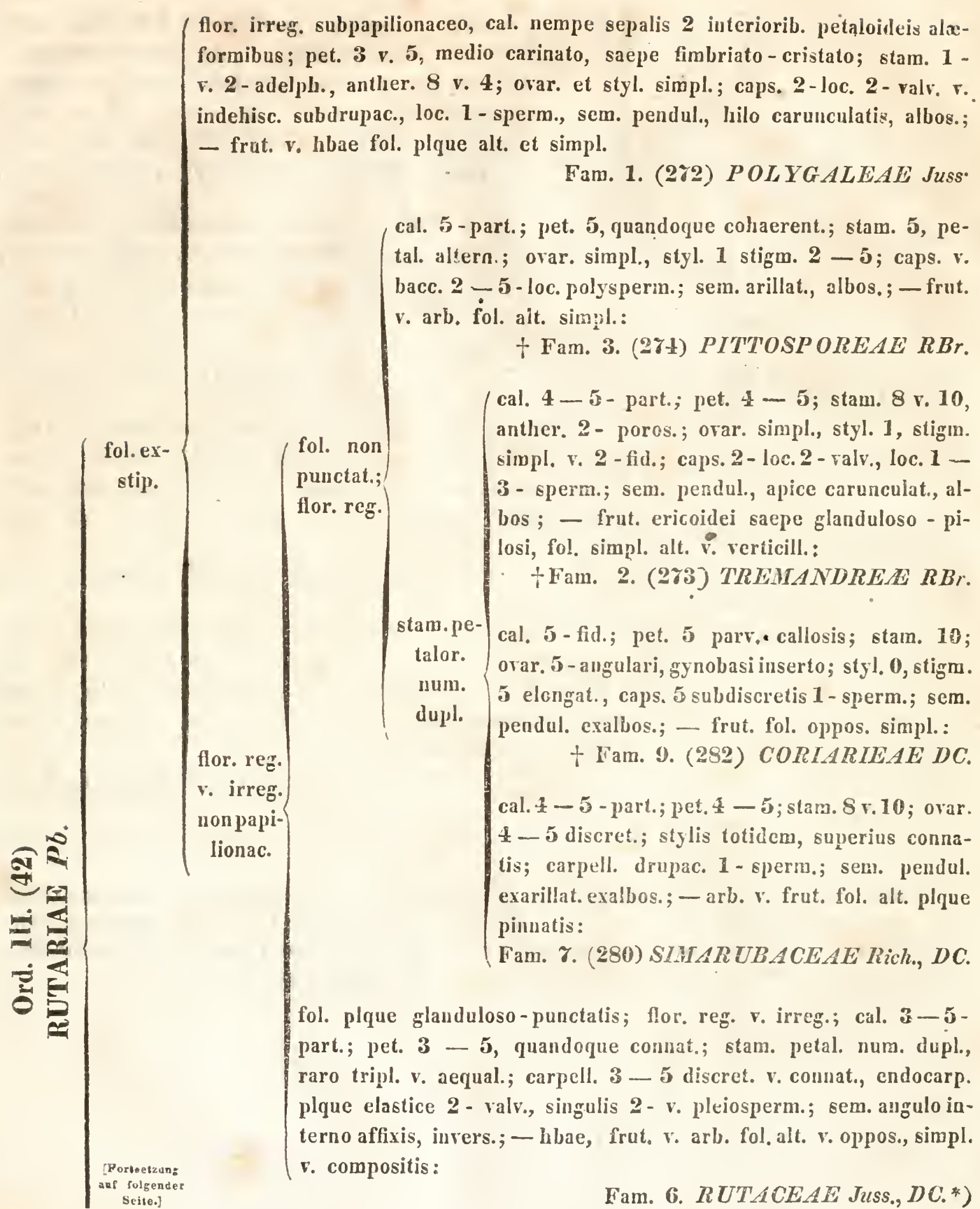

Seite.]

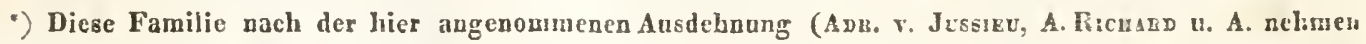




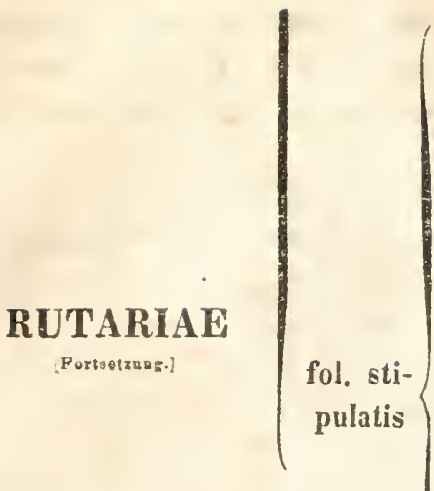

flor. plque reg; cal.5-part.; pet. 5; stam. plque 10; orar. simpl., styl. plque 5-fid.; caps. 5-loc., loc. 1 - v. pleiosperm.; sem. plque pendul.; - hbae, frut. . arb. fol. oppos. plque pinnat, non punctatis:

Fam. 5. (276) ZYGOPHYLLEAE RBr.

flor, reg.; cal. 5-v. 4-part.; pet. 5, 4 v. 10; stam. totidem r. hoc num. dupl. v. indefiı., anther, 2 -poros.; orar. 5 v. 10 discret., gynobasi tumidae insertis; styl. 1, medio; carpell. 10, $5 \mathrm{v}$. abortu pauciorib., subdrupac, 1 - sperm.; sem. erect.; - arb. v. frut. fol. alt. simpl.:

† Fam. 8. (281) OCHNACEAE DC.

flor. reg.; cal. 5- part.; jet. 5; stam. totiden, urceolo dentato iusert.; orar. et sty!o simpl.; fruct. drupac. 5-1oc. polysperm., sem. angulo interno affix., exalbos.; - arb. trunco subsimpl., fol. alt. simpl, coriac. non punctat., stip. parv. caducis :

† Fan. 4. (275) BREXIACEAE Ldl.

auch noch die Zygoply $l l e$ en und Simarubeen darein anf) zcrällt in drei Gruppen, welche manchen Syotematikern als eben so viele Familien grelten:

RUTACEAE $D C$

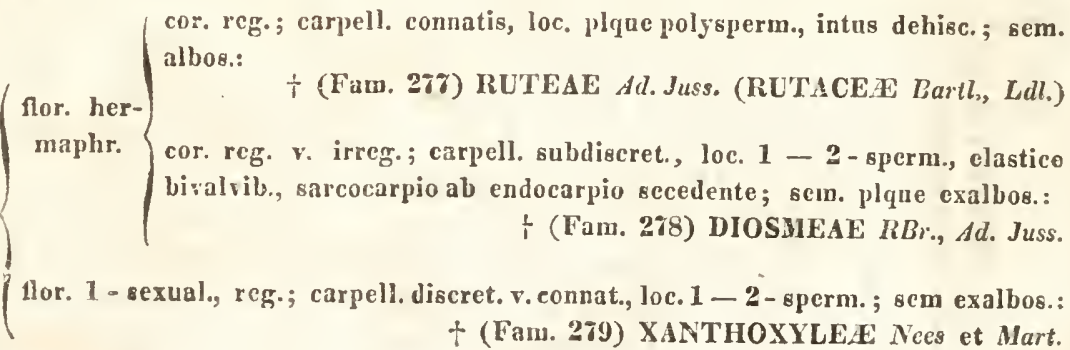


pet. 5, intus squama auctis; stam. 10 basi 1 - adelph.; ovar. sub-3 loc., Ioc. 1 - orul.; styl. 3 v. 1 ; drupa 1 - sperm. sem. pendul. albos, embr. rect,; - frut. r. arb. fol. plque alt., simpl., stip. axillar.:

Fam. 1. (283) ERYTHROXYLEAE Tth. petal. pet. 5 (raro 4 v. 6); stam. 3 (raro $4-5$ ), filament. basi laia in urceolum comnatis; ovar. 8 -loc., loc. 2 - pluriovul; siyl. 1 stigm. simpl. r. -3 lobo; fruct. v. caps. 3-1 6amaroid. 2-valv, v. baccat. 1 - 3 -loc, sem. erect., exalbos., embr. rect.; - frut. arboresc. v. scand. fol. oppos. simpl., stip. parv. decid.:

† Fan. 2. (29\$) HIPPOCRATEACFAE Juss. am. plus minus connatis

flor. irreg. cal. cal-

arb. $v$. frut., fol.

calyc. non. calcarat. (fol. exstip.)

carat.

Fam. 4. (286) MALPIGHIACEAE Juss. pet.4-9, raro 0; st am. 8 v, raius 5-12, lib; orar. plque didymo; styl. 1 , stigm. 2; fruct. carpell. 2 r. raro 3 samaroid. 1 - 2 sperm.; sem. erect. exalbos., enbr. curr.; - arb. fol. oppos. simpl. v. comp., exstip.:

Fam. 3. (285) ACERINEAE DC. pet. 5. 4 ; stam. $f$ v. 8 inaequal., lib.; orar. 3 -loc., loc. 2 - orul.; styl. 1 ; caps. coriac. 1 -3-vals. 1 -3 loc. 1-3-sperm.; sem. magn. exalbos., embr. curv.:

Fam. 5. (287) HIPPOCASTANEAE DC. oppos. pet. 5 crassiuscul. subinaequal,; stam. numeroz. duplici digitat. serie, basi breviter connat.; styl. 4 ; fruct. e nucib. 4 coadunat. (quibusdam saepe abortientib.) 1 - sperm., sem. exalbos.:

? † Fam. 6. (288) RHIZOBOLEAE DC. arb., frut. v. hibae, saepe scand., fol. alt. plque pinnat. v. tern,; pet. tot quot. lob. cal. v. 1 deficiente (raroo), saepe intus lamina auctis $v$. glandulos. v. villos.; stam 8 v. 10, raro plurib. v. plauciorib., lib., plque asymmetr.; styl. 3 v. 1; fruct. caps, saepe inflata v, alat., v. drupac. plque 3 - loc., loc. 1 - sperm., sem. exalbos.:

Fain. \%. (289) SAPIND ACEAE Juss.

pet. 5, inaequal., calyci insert.; stam. 8 ; styl, 3fid.; caps. subbaccat. subiricocca, loc. 1 - spern.; sem. exalbos.; - hbae volub. v. diffusae, fol. alt. peltat., exstip.:

Fam. 8. (290) TROPAEOLEAE Juss.

pet. 1 - 3, raro 5, inaeq., imo calyci insert.; stam. $1-5$, plque unico antherifero; styl. 1 simpl.; caps. 3 - gon. 3 - loc. 3 - valv., loc. 1-v. pleiosperm., sem. exalbos.; - arb. fol. oppos. v. verticill. simpl., stipulat.:

? + Fam. 9. (291) VOCHYSIACEAE St. Hil., Mart. 


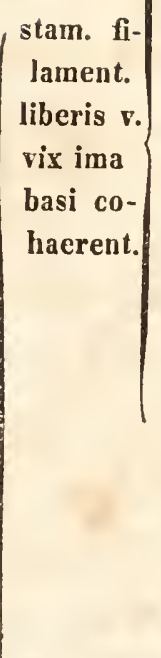

Ord. V. (44)

COLUMINIFER $A$

Linn. e. e. Pb. pet. $4-5$, apice lobat. v. fimbriat.; stam. 15- 20 lib., anther. elongat., apice 2 - poros.; styl. 1 ; fruct. capsular. v. subdrupac. 1-5-loc., loc. 2 - v. pleiosperm.; - arb. จ. frut. fol. alt. simpl., stip. decid.:

† Fam. 1. (292) ELAEOCARPEAE Juss.

pet. $4-5$, raro 0 ; stam. plque indefin., anther. intrors.; styl. 1, stigm. plque partit.; fruct. capsular. pluriloc., loc. 1 - v. pleiosperm.; sem. albos.; - arb. v. frut., raro hbae, fol. simpl.:

petal. integr.; anther. 2 - ri- pet. 5; stam. indefin., subpolyadelph.; ovar. plurimos.

Fam. 2. (293) TILIACEAE Juss. loc., loc. 2 - ovulat.; styl. et stigm. 1; fruct. coriac., abortu 1 - loc. 3 - valv. v. indehisc., 1 -sperm.; senı. exalbos.; - arb. fol. vernatione iuvolut., stip. decid.:

Fam. 6. (297) DIPTEROCARPEAE Bl.

cal. 3 - part,; pet. 5 v. 0 ; stam. defin. v. indefin., anther extrors.; ovar 5 -v. rarius 3 -fid.; styl. 5 v. 3, quandoque connat.; fruct, caps. plque 5 - loc., loc. 1 - v. pleiosperm.; sem. apice carunculat., pique albos:; - arb. v. frut. fol. plque alt., simpl. anther. 2 - r. raro digitat.:

loc.

Fam. 3. (294) BÜTTNERIACEL RBr., DC. cal. 3 - sepal. parv.; pet. 5. v. 6; stam. indefin. v. 10; ovar. simpl. 3 - loc; styl. 1, stigm. 3 -fid.; fruct. caps. 3-v. 1 -loc., loc. 1-v. pleiosperm.; sem. albos.; - arb, vo frut. fol. alt. simpl. integr. stip. decid.:

? † Fam. 7. (298) CHLENACEAE Pet.- Th.

stam. fi-

lament. connatis

anther. 1 loc. (cal. pique calyculat.; pet. 5 , basi cum stan. connat.) adelph.; fruct. caps., bacc. v. drup.; sem. saepe stam. filamentis basi 1 -adelph., superne sub-5 lana v. pulpa involut., albos. v. exalbos., cotyled. plque corrugat. v. couvolut.; - arb. v. frut. fol. alt. simpI. v. digitat.:

Fam. 4. (295) BOMBACEAE Kth.

stam. filament. penitus 1 - adelph.; styl. v. stigm. numeros.; fruct. caps. saepe verticill., raro baccat.; sem. quandoque lanat., exalbos., cotyled. chrysaloideo - contortuplicatis; - hbae, frut. $\nabla$. arb. fol. alt. simpl., saepe lobat.:

Fam. 5. (296) MALVACEAE Juss, e. e. Kth. 


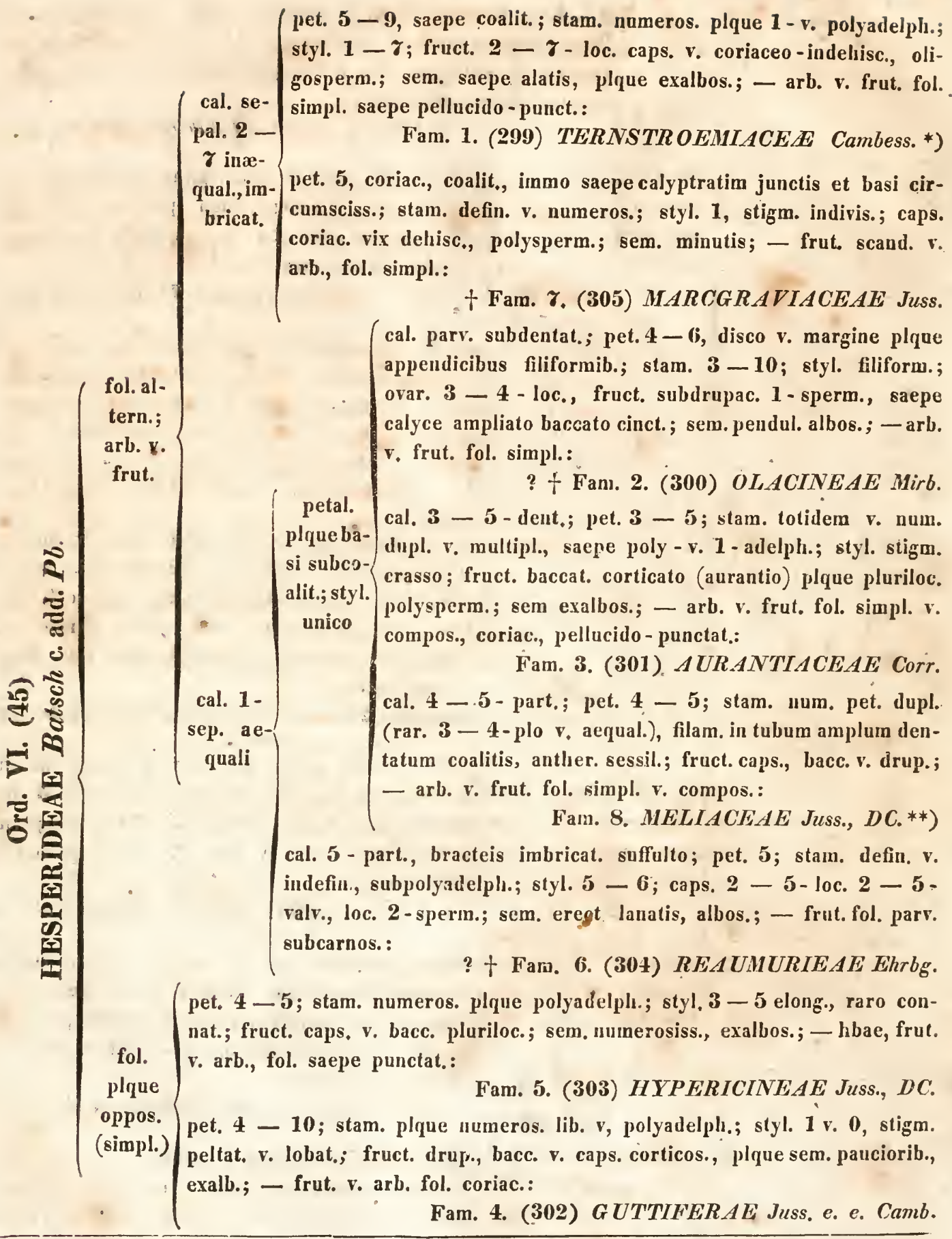

-) Die durch Mirbec und Decandole aufgestellten Familien der Ternetroemie a e Mirb. und Camellieac DC. (The acea Mirb.) sind durch keine haltbaren Merkmale unterschieden nnd werden daher nach Casrbessides's Vorgange hier vereint.

•) Hier sind zweifelsohne noch Trennungen vorzunehmen, doch dürfte bis jetzt nur jene der CedreI e a o sich ganz sicher charakterisiren lassen: 
sal. parv. subdentat.; pet. 5 r. 4 , basi latis, quandoque apice v. basi coliaerent.; stam. 5 v. 4, pet. oppos.; styl. 1 v. 0; bacc. globos. 1 - 5-sperm.; sem. albos.; - frut, plque scand., fol. oppos. v. alt., simpl. v. compos., stipulat.:

Fam. 1. (308) VINIFERAE Juss.

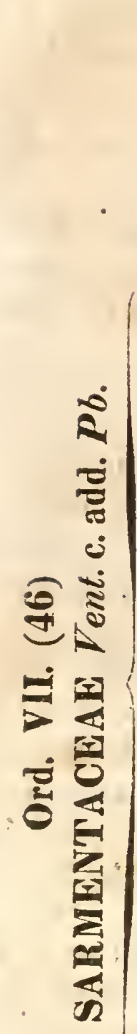

fl. irreg.;

flor. plque regul.; cal. flor. reg.; ovar. simpl.

calcare licul., basi quandoque cohær., stam. sub -

1 -ade!pl.; sepal. 5 - 3 ; pet. totidem.; stam. totidem fertistyl.5(v.ra- lib.; caps. 10 - 6 - loc., loc. 1 - sperm.; sem. alb. ro 4 v. 3) subo; - hbae v. suffrut. fol. alt. $\nabla$ oppos. simpl. integerr., exstip.:.

\section{Fam. 3. (310) LINEAE DC.}

flor. reg. v. irreg.; cal. sepal. 5, uno quandoque in calcar tubuliforme pedunculo penitus adnatum producto; stam. 10 v. 15, basi 1 -adelph., quibusdam plque steril,; ovar. 5 columnae centrali elongatae affix; caps. 5, monosperm.; sem. exalbos.; - hbae v. suffrut. caule artic. nodoso, fol. oppos. v. alt., stipulat.

cal. libere calcarato, plque 5 sep. colorat.; stam. 5 , anther. subconnat.; stigm.ós sessil.

Fam. 4. (311) GERANIACEAE Juss., DC.

pet. 5 inaequal.; drup. 5-pyren.; sem. exalbos.; - hbae fol. alt. exstip.: Fam. 5. (312) HY.DROCEREAE Bl.*)

cal. sepal. 2 saepe deficientib.; petal. 5 inaequal., lateralibus binis connat., superiore galeato; caps. elastice 5 - valr. polysperm.; sem. exalbos.; - hbae fol. alt. $v$. oppos. simpl. exstip.:

Fam. 6. (313) BAISSAMINEAE,A. Rich. **)

MELIACEAE $D C$.

(ovar. et fruct. baccat., drupae; v. capsular. $2-5$-, raro $10-20$ - Ioc., loc. 1 - 2-serm.; scm. non alatis; - arb. v. frut. fol. simpl.v. compos.: $\dagger$ (Faın. 306) MELIACEAE DC. e. e. RBr. ovar. et fruct. capsular. 5 - v. raro $3-4$-loc., loc. pleiosperm.; $8 \mathrm{~cm}$. alatis; arb. fol. pinnatis:

† (Fam. 307) CEDRELEAE RBr.

-) Vielleicht würde, wie v. Mátius andeutet, diese Fannilie besser nit der folgenden verbunden.

-) Nachdem Röper die pentamerische Bildung der Balsaminenblüthe, gegen Decaxpocte und Agandi, entscheidend nachgewiesen lıat, mufs diese Familie lier, anstatt in der folgenden Ordnung, wohin ich sic frülıer gestellt batte, ihren Platz nehmen. 
cal. 4 sepal. v. 4 - part.; sem. exalbos.

\section{Ord. VIII. (4\%)} CRUCIFLORAE Pb.

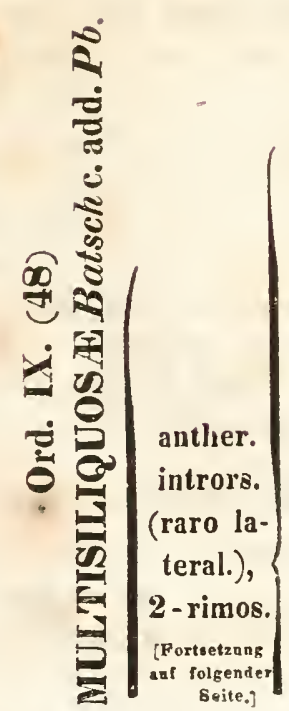

stam. 6 tetradynamis; fruct. 2 -loc. 2 - valv. siliquos, v. nucamentaceo v. raro transversim plurilocul. lomentac.; - hbæ $\mathrm{r}$. suffrut. fol. simpl. . compos.:

Fam. 1. (314) CRUCIFERAE Juss. stam. defin." $\nabla$. indefin. (rariss, tetradynam.); ovar. stipitat.; fruct. 1 - loc. siliquos. 2 - valv. v. baccat.; - hbae, frut, v. arbusc., quandoque spinos., fol. simpl. v. digitat.:

Fam. 2. (315) CAPPARIDEAE Juss., DC.

(cor. irreg., petal. plque subcoalitis, exteriore uno v. duobus saepe basi saccato-calcar.; stam. 6 diadelph.; fruct. 1 -loc. siliquos. 2-valv. polysperm., v. nucula $1-2$-sperm.; - hbx tenerae, quandoque scand., fol. multisectis:

Fam. 3. (316) FUMARIACEAE DC. sepal.caduco; sem. albos.

car. reg., petal. 4, 8, 12; stam. totidem v. saepius numeros., lib.; fruct. 1 - loc. capsular. v. siliquos. polysperm.; - hbae v. suffrut., plque lactesc., fol. simpl. v. compos.:

Fam. 4. (317) PAPAVERACEAE Juss., $D C$.

sepal. 4-6; pet. et stam. numeros. pluriseriat.; styl. plurib. stigm. simpl., v. styl. O stigm. sess. stellat.; carpell. $8-2 \pm$ toro obconico truncato semiimmersis v. urceolato penitus inclusis; sem. embr. intra sacculum proprium; - hbae fol. peltat. $\nabla$. cordatis: pente; fol. longe petiol.

Fam. 2. NYMPHAEACEA Salisb., DC.*) plque natantib.; flor. sepal. 3 - 4; pet. totidem.; stam. 6-36; orar. 2 v. plurib., styl. totid. simpl.; carpell. 1 - 2 -sperm. indehisc.; sem. embr. in cavitate basilari alb. sito; hbae fol. omnib. v. summis peltatis:

† Fam. 3. (321) CAMBOMBEAE Rich.

sepal. 5; pet. 5 unguicul.; stam. indefin.; orar. et styl. 1, stigm. magno peltato; caps. 5-loc. polysperm., placent. 5 central.; sem. albos.; - hbae acaules fol. radic. utriculos. opercul.:

? + Fam. 1. (318) SARRACENIACEA Lapyl.

palustres

sepal. 3 - 4; pet. 6 - 9; stam. $12-18$; ovar. 1 , styl. sub0, stigm. crasso subpeltato; caps. v. bacc. 1 loc. polysperm., placenta unica parietali; sem. albos.; - hbae fol. lobatis:

Fam. 4. (322) PODOPHYLIEAE DC., Ldl.

") Dem Fruchtbane nach wåren hier eigentlich, wie Barturic, Lindegy u. A. getban haben, zu trennen: 


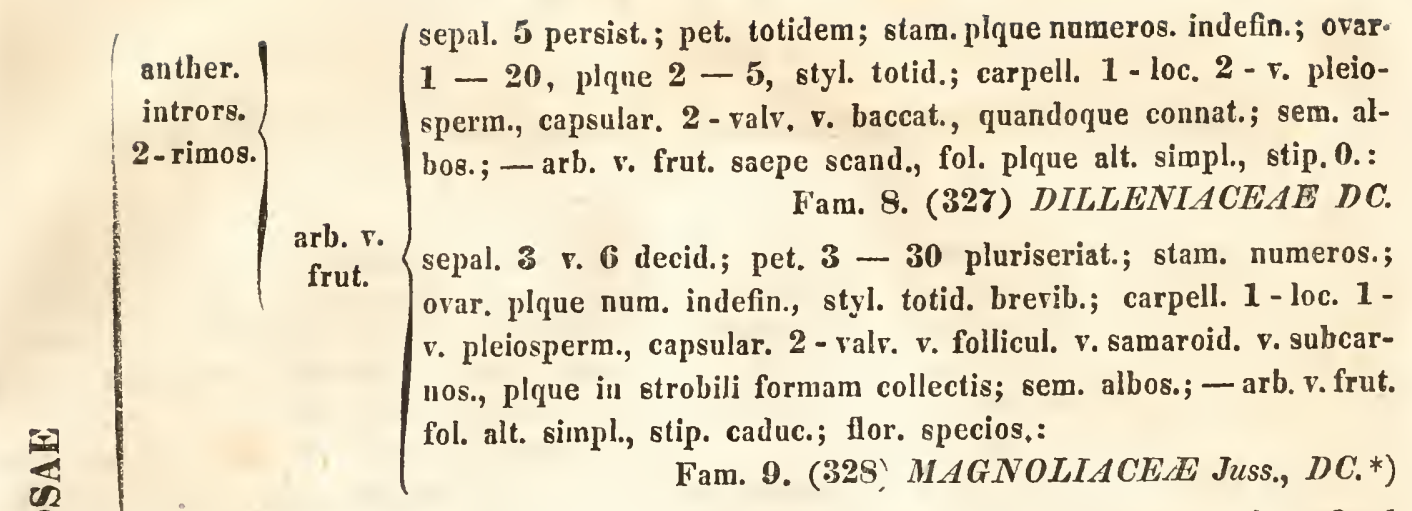

antheris lateralib. a basi ad apicem valrula dehisc.; sepal., pet. et stam plque 6, 4 v. raro 3, omnibus invicem oppos.; ovar. 1 stylo brevi, stigm. orbicul.; fruct. baccat. v. caps. 1 -loc. oligosperm.; sem. albos.; - frut. v. hbae fol. alt. simpl. v. compos., quibusdam saepe in spinas mutatis; stip.0:

\section{Fam. 5. (323) BERBERIDEAE Vent., DC.}

(flor. 1 - sex., plque dioic., parvis; sepal. et pet. $3,6,9$ v. 4, 8, 12; stam. totidem v. 3-4-plic. num., 1 -allelph. v. rar. lib.; ovar. plurib. 1 -stylis v. unico stigmatib. plurib.; carpell. baccat. v. drupac. 1 - raro 2 - v. pleiosperm.; sem. subexalbos.; - frut. sarmentosi, fol. alt. simpl. v, rar. compos., apice mucron.; stip.0:

Fam. 6. (32t) MENISPERMACEAE Juss., DC.

antlier.

plinue

extrors.

2 -rimos. flor.

plque

hermaplirod.; stam. indeîn. li-

beris flor. reg. v. irreg., perigon. num. plque 5-v. 4-nar., sepal, saepe colorat., quandoque calcarat. $v$. galeat.; pet. sacpe varie transform. จ. 0 ; ovar. et curpell. plque numeros. lib. 1 -sperm. 1 -stylis, $\nabla$. pauciorib. subconnat. 2 - polysperm., rariss. unico; fract. caryopsid., caps. v. rar. baccat,; sem. albos.; - hbae fol. radical. v. alt. v. rar. oppos., simpl. v. compos., rarius frut. sarment., fol. oppos.; stip. 0 :

Fam. 7. (325) RANUNCULACEAE Juss., DC.**)

flor. reg., perigon. num. plque 3 - nar.; pet. 6 biseriat., saepe coriac.; ovar. et carpell. plque numeros. (rariss 3 v. 1) lib. v. connat., 1-v. pleiosperm.; fruct. baccat. $\nabla$, rarius siccis capsular.; sem. albos.; - arb. v. frut. fol. alt. simpl, stip. 0:

Fam. 10. (330) ANONACEAE Juss., DC.

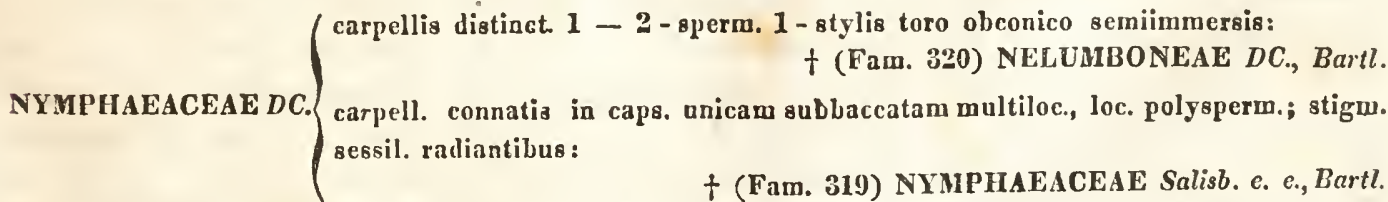

-) Einige Auctoren trennen von dieser-Familie die ( + Fasa. 329) WINTEREAE RBr. (ILLICIEAE DC.) wegen der drüsig punctirten Blätter.

-) Dicae Familic ist in allen Hinsichten sehr schwierig durch Charaktere zusammenzufassen und dennoch unstreitig naturgemäfs; nur dic durch antherae introrage anterschiedenen († Fam. 326) PAEONIACEAE DC. sollten viclleicht getrennt werden. 


\section{$R$ e $g$ is $\mathbf{s}$ e $\mathbf{r}^{*}$ \\ der}

\section{Classen, Ordnungen und Familien der Pflanzen.}

NB. Die hier angegebenen Zahlen beziehen sich auf die fortlaufcnde Numerirung im vorangehenden Cla. vis, also bei den Ordnungen und Familien auf die in Parenthesen beigcsetzten Nummern. Mit dem Zeichen= werden gleichbedeutende Bcnennungen auf jene des Clavis reducirt.; mit $<$ werden Gruppen, welche kleiner, und mit > Gruppen, wilche gröfser, als die durch die beigesetzte Nummer angezeigte, jedoch hierin gans oder theilweise enthalten sind, bezeichnet. Cl. bedeutet Classe, O. Ordnnn , Fam. Familie.

Abietinae Rich.

Fam. Acanthaceae Juss., RBr. 131 Acerineae DC. 285

Acorinae Lk.

Acoroideae Ag. ACOTYLEDONEAE JUSS., DC.

Cl. I. et II. Aegicereae $\mathrm{Bl}$. $<12 \gamma$ Aggregato Linn., Pb. O. 30. Alangieae DC. Alg ae Juss., Roth. 0.3. Algae Linn. = 0.2 et 3. Alismaceae Rich.

Alismaceae Ag.

Aloinae Lk.

Alsineae DC., Bartl.

Amarantaceae Juss., RBr. 10ذ Amaryllideae $\mathbf{R B r}$. Ambrosiaceae Cass.,

Schultz. $?<90$ Amentacece Juss., Pb. 0.16. Amomeae Rich., Bartl. $<76$ Ampelideae Kth. $=305$ Amygdaleae Juss., DC. $\quad 226$ Amyrideae Kth. 213 Anacardiaceae Ldl. Anacardieae DC. $>219$ 219 Andreacaceae Nees. Anemoneae DC. Angiogasteres Nees. Anonaceae Juss., DC. Anthemideae Cass. Anthoceroteae Nees. Anthoboleae Mart. $<3$ 330 $<187$ Anthospermeae Cham. et Selıl.
Apocyneae Juss., DC. $\quad$ Fam. Apostasieae Lell.

78

Aquifoliacene DC., Bartl. $<210$ Aquilarineae $\mathrm{RBr}$.

112 Araliaceae Juss., DC.

201 Arctotideae Cass. Ardisiaceae Juss. $<187$

128 Arecaceae Schultz

$=61$ Arecinae Mart.

61

Aristolochieae Juss., Spr. $>121$ Aroideae Juss., Ag.

36 Artocarpeae DC., RBr. Asarineae $\mathbf{R B r}$. Asclepiadeae $\mathrm{RBr}$. Asparageae Jiss., Ag. Asphodeleae Juss., Ag. Astereae Cass.

160

$6 \pm$

66 Asteroideae Lessg. Ataxae Pb. 0. 28. $<187$ Atherospermeae $\mathrm{RBr}$. Atriplices Juss. Aurantiaceae Corr.

Balanophoreae Rich.

118

Balsamifluae BI.

Balsamineae A. Rich.

Barringtonieae DC.

313

Batideae Mart.

232

Batrachospermeas Ag.

97

Baueraceae Ldl.

Begoniaceae Bonpl.

Belvisiaceae $\mathbf{l} \mathrm{Br}$.

Berberideae Vent., DC.

$<12$

250

108

199

323

Betulaceae Rich.

81

Bifolliculares Mart. $<161$ Bignoniaceae Juss, RBr. $\mathbf{I 3 3}$
Fam.

Bixineae Kth., DC. 265 Bombaceae Kth. 295 Boopideae Cass. $\quad=\mathbf{1 8 3}$ Borasseae Mart. 60 Borragineae Juss., Vent. $\mathbf{1 4 3}$ Brexiaceae Ldl. 275 Bromeliaceae Juss. 56 Bruniaceae $\mathrm{RBBr}$. 208 Brunoniaceae Lull. $\quad 192$ Bryaceae Bartl. 21 Bucideae Spr. $\quad=110$ Büttneriaceae RBr., DC. 294 Burmanniaceae Bl, Ldl. 73 Burseraceae Kth. . 215 Butomeae Rich. $\quad 52$ Byssaceae Nees. $\quad<2$ Byssi Eudl. $<2$

Cabombeae Rich. 321

Cactcae DC. $=2 \frac{4}{45}$

Caesalpiniaceae DG.,Pb. 222 Calameae Ktli. 59 Calendulaceae Cass. $<18 \mathrm{r}$ Callaceae Bartl. $\quad><3$ Callitrichinae Lk. $\quad 35$ Calojhylleae Nart. $<302$ Calycantilze Pb. Cl. VII. Calycantheae Ldl. $=\mathbf{2 3 3}$ Calycereae RBr. $\quad 188$ CaLYcopetalas Pb. Cl. VIII. Camellieae DC. $<299$ Campanulaceae Juss., Spr. 194 Canellaceae Mlart. $\quad ?<\mathbf{3 0 6}$ Cannaceae RiBr. $<$ gr Camnae Juss. $\quad=\boldsymbol{\gamma} \mathbf{0}$ Capparideae Jus8., DC. 315 
Fam.

Caprifoliaceae Bartl. $=178$ Caprifoliacene Juss., DC. $>\mathbf{1 7 8}$ Carduaceae Ktlı., Cass. $18 \pm$ Cariceae Turp. $=196$ Carisseae Spr. $>162$ Carlineae Cass. $<184$ Carpocereae Dumort. $=\mathbf{1 7}$ Caryophyllea Juss., DC. O. 40.

Cassieae DC. $<222$ Cassuvieae $\mathbf{R B r}$. $>219$ Cassytheae Bartl., Ldl. $<\mathbf{1 1 6}$ Casuarineae Mlirb.

Cedreleae RBr. Cedrinae $\mathbf{P b}$.

Celastreae $\mathbf{R B r}$., $\mathbf{P b}$.

Celastrineae RBr., DC. $>\mathbf{2 1 0}$ CELLULares DC. Cl. I et II. Cellideae Rich. $=88$ Centaurieac Cass. $<181$ Centrolepicleae Desr.

Cephaëlideae Cham. et Schl. $<178$ Cephalantheae Juss., Pb. $\mathbf{1 7 \gamma}$ Cephalodeae Ag. Cephaloteae Lil. $?<230$ Cephalotheceac Dumort. $=18$ Ceratophylleae Gray. 33 Cercodianae Ju. . $\quad=\mathbf{2 5 3}$ Cestrinae Schlchtd. $\quad<1 \Vdash 3$ Chailletiaceae IBBr., DC. 212 Characeae Rich.

Chenopodieae DC., Bartl. 103 Cimonantheae Pb.

233

Chlenaceae Pet.- Th. 298 Chlorantheae $\mathrm{RBr}$.

101

Chrysobalaneae RBr.

225

Cichoraceae Vaill., Juss. $=\mathbf{1 8 8}$

Cinchonaceae DC.

176

Cinchoneae Cham. et

Sch.

Circceaceae LdI.

Cisti Juss.

$<238$

$>26 \pm$

Cistineae DC. $26 \pm$

Cliffortiaceae Mart. ? $<230$ Cobaeaceae Don.
Coccocypselece Cham. et Schl.

Cocoince Mart.

Coffeaceae DC., $\mathbf{P b}$.

Colchicaceae DC.

Columelliaceae Don. ? $<\mathbf{1 2 9}$

Columnifera Linn., $\mathbf{P b}$. O. 44.

Combretaccae RBr.

234

Commelineae RBr.

49

Compositae Vaill., Linn., DC. $<0$. 31 .

Confervaceae Ag., Endl. 12

Confervoideae Ag. $\quad=\mathbf{1 2}$

Coniferae Juss., Bartl. O. 15

Coniomycetes Fr.

Connaraceae RBr., Kth. 214

Contoriae Linn. $>160,161$

Convolvulaceae Bartl. $\mathbf{1 5 0}$

Convolvulaceae Vent. $>\mathbf{1 5 0}$

Cordiaceae RBr., Mart. 1 ty

Cordiereae A. Rich. $<\mathbf{1 7 5}$

Coriarieae DC.

282

Corneae DC.

202

Coronariae Ag.

67

Corymbiferae Juss. $=185$ -

Corynomycetes Mart. $<5$

Coryphinae Mart. 58

COTYLEDONEAE DC. Cl. III et $s q q$.

Crassulaceae DC.

Cruciferae Juss.

$31 \pm$

Cruciflorae $\mathrm{Pb} .0 .47$.

Cryptogamae Linn. $=\mathrm{Cl}$. $I-I I T$.

Crustaceae Ag.

Cryptocotyledoneae $\Lambda \mathrm{g} .=$ Cl. IV.

Cucurbitaccae Juss., Bartl. 195

Cunoniaceae $\mathbf{R B r}$.

251

Cupuliferae Rich.

85

Cupressinae Rich.

Cuscuteae Presl.

Cyatheaceac Mart.

Cyathinae Pb. 0. 22.

Cycadeac Pers.

79
Cyclantheae Poit.

Fam.

Cynareae Spr., Lessg. $=184$

Cynarocephalae Vaill., Juss. 184

Cyperaceae DC.

43

Cyperoidene Juss.

$=43$

Cyrtandraceae Jack.

134

Cytineae Brongn., Ldl. 119

Dinaeaceae Ag.

27

J)at isceae $\mathrm{RBr}$.

Delimaceae DC. 327

Desvanxieae Bartl. $\quad=\mathbf{4 4}$

Detarieae DC.

$<222$

Diatomaceae Ag., Endl.

11.

Diatomeae Ag.

$<11$

Declidopterides Kaulf. O.6.

DIcotrledoneaE Juss., DC.

Cl. V el sqq.

Didymocarpeae Doll. $=\mathbf{1 3 4}$

Dilleniacene DC. 328

Dioscoreaceae $\mathbf{R B r}$.

Diosmeae RBr., Ad. Juss. 278

Diospyreae DC.

$=166$

Dipsaceae Juss., DC. 182

Dipterocarpeae Bl.

297

Discoideae Linn.

185

Dombeyaceae Kth. $\quad<\mathbf{2 9 4}$

Droseraceae DC. $\quad \mathbf{2 6 8}$

Drupaceae DC., Spr. $>\mathbf{2 2 6}$

Dryadeae Vent. $\quad=\mathbf{2 2 9}$

Drymyrrhizeae Vent. $=\boldsymbol{\gamma 6}$

Ebenaceae Vent., RBr. 166

Echinopeae Cass.

184

Ehretiacene Mart.

146

Iilcagneae Juss., A. Rich. 111

Elaeocarpeae Juss. $\quad 292$

Elatineae Cambess. $\quad \mathbf{2 6 0}$

Elegieae Beaur. $=\mathbf{4 5}$

Empetreae Nutt. 208

EvDOGENAE DC. Cl. IIIet IV.

Endorrhizae Rich. $=C l . I V$.

Ensatae Linn.

$>7 \pm$

Entocarpae $\mathbf{P b}$.

Entophyta Lk.

Epacrideae RBr. 
Fam.

Epilobiaceae Vent. $=238$

Epiphyllospermœ Ray. O..

Equisetaceae DC.

30

Ericaceae DC.

$>169$

Ericeae RBr.

169

Eriocauleae Mart.

47

Erythroxyleae Kth.

283

Escallonicae RBr.

$2+9$

Eupatoriaceae Lessg.

Eupatorinae Spr.

Euphorbiaceae Juss.

$<185$

$=185$

96

Evonymeae DC.

$=\mathbf{2 1 0}$

EXoGENAE DC. Cl. V et sqq.

Fagopyrinae Bartl.

$>106$

Ficoideae Juss.

256

Fílices Linil. $=C l$. III.

FILICINAE Pb. Cl. III.

Filicoideae Ldl. $=C l$. III.

Flacourtiaceae A. Rich.

Florideae Lmrx.

Fluviales Vent.

$<15$

Fothergilleae DC.

$=32$

Fouquieraceae DC., Kth. 280

Fragariaceae Rich. $\quad=229$

Francoaceae Ad. Juss. $=25 \pm$

Frankeniaceae St. Ilil. 263

Fraxineae Mart. $\quad<13 \gamma$

Frondosi Musci Hedw. 0.5.

Fucaceae Lmrx., Pb.

15

Fucoideac $\mathbf{\Lambda g}$.

Fumariaceae DC.

Fungi Linn., Juss. O. 1.

Galacineae Don.

$25 \pm$

Garcinieae Bartl. $=302$

Gardeniaceae $\mathbf{\Lambda}$. Rich. $<>\mathbf{1 7 6}$

Gardnereae Wall.

Gasteromycetes Fr.

Gentianeae Juss.

Gentianeae Mart.

Geoffreae DC.

$16 \pm$

3

$>156$

156

Geraniaceae Juss., DC.

221

311

Gessneriaceae Rich., Lk. 13 J

Gilliesieae Ldl.

$?<66$

Gleicheniaceae RBr., Mart. 25
Glinoideae Mart.

Fam.

Globularineae DC.

256

123

Glumaceae Pb. O. 10.

Gneteae BI.

$?<81$

Goodenovieae RBr., Ldl. 190

Gordonieae DC.

$?<299$

Gramineae Juss.

42

Granateae Don.

$<232$

Grossularieae DC.

$2 \pm t$

Guajacanae Juss.

$=160$

Guettardaceae Kth., Cham.

et Schl.

$<17.5$

Guttiferae Juss., Cambess. 302

Gymnomycetes Lk.

$=1$

Gynandrae Ag. O. 11.

Gyropterides $\mathrm{Pb}$.

$=26$

Haemodoraceae $\mathrm{RBr}$.

Halesieae Don.

72

Halorageae RBr., Bartl.

168

Hamamelideae $\mathbf{R B r}$.

253

Hamelieae A.Rich., Kth. $<\mathbf{1 8 0}$

Hederaceae Linn., $\mathrm{Pb} .>202$

Hedyotideae Cham. et

Schl.

Heliantheae Cass.

$>176$

Heliotropieae Sclırad.

$<$ is8

Hemerocallidede $\mathbf{R B r} . \quad<\mathbf{6 r}$

Ilepaticae Hedw., Juss. O.4.

Hepaticae Willd. $<0.4$.

Hermanniacece Juss., Kth. $<29 \pm$

Hernandieac Bl. $?<\mathbf{1 1 5}$

Hesperideae Batsch., Pb.

$$
0.45 .
$$

Hippocastaneae DC.

Hippocrateaceae Juss.

Hippurideae Lk.

Homalineae RBr.

Homalophyllae Willd.

Humiriaceac Ad. Juss.

Hydrangeaceae Ldl.

Hydrocaryes Lk.

Hydrocereae $\mathbf{B l .}$

287

$28 \pm$

Hydrocharideae Juss.,

RBr.

Hydrocharideae Lk.

312

$5 \pm$
Hydroleaceae $\mathbf{R B r}$.

Hydropeltideae DC. $=\mathbf{3 2 1}$ Hydrophylleae RBr.,

Schrad.

149

Hydrophyta Lyngb. $=0.3$.

Hydropterides Willd. $=\mathbf{2 8 ,} \mathbf{2 0}$

Hygrobiae Rich.

$=253$

Hymenomycetes Fr.

Hymenophylleae Mart. $<\mathbf{2 0}$

Hypericineae Juss., DC, $\$ 03$

Iyphomycetes Lk., Fr.

2

Hypoxideae $\mathrm{RBr}$.

68

Hypoxyla БC.

$=4$

Ilicineae Brongn.

$<210$

Illecebreae RBr., Ldl.

258

Illicieâ $\mathbf{D C}$.

Inomycetes Fr.

Inuleae Cass.

Ionidieae Spr., $\mathbf{P b}$.

Irideae Juss.

$<328$

Isertieae A. Rich.

Isoëteae Ricl.

$<2$

187

266

Jacobeae Kth.

74

Jasmineae Juss., RBr.

176

29

Juglandeae DC.

187

136

Juncaceae Ag.

220

Juncagincae Rich.

$=46$

Junceae DC.

50

Junci Juss.

40

Jungermaniniaceae Nees,

Dumort.

19

Krameriaceae Ktl.

$<272$

Labiatae Juss.

142

Lacisiemeae Mart.

102

Lactuceae Cass.

$=189$

Lasiopetaleae Gay.

$<294$

Laureolinae Pb. O. 20.

Laurineae Juss., RBr. 116

Lecythideae Rich. $\quad<232$

Leeaceae DC.

$?<308$

Leguminos ae Juss. 0.36 .

Lemnaceae DC.

Lentibulariae $\mathrm{RBr}$.

Lepidocarya Mart. 
Lichenes Achar. O. 2.

Ligustrinae Bartl. $>137$

Liliaceae Adans., Pb. O. 13.

Lineae DC.

310

Lingulatae Ponted.

Loaseae Juss.

188

$2+8$

Lobeliaceae Juss, RBr.

Lobiolatae Ag.

193

7

Loganiaceae. Bartl., $\mathbf{P b} .>158$

Loganieae RBr., Mart.

158

Lonicereae $\mathbf{R B r}$.

178

Lorantheae Rich., DC. 203

Luridae Linn., Pb. O. 26.

Lycoperdaceae Brongn. $<\mathbf{3}$

Lycopodiaceae DC.

22

Lygodysodeaceae Bartl.? < 185

Lysimachine Juss.

$<126$

Lythrariae Juss.

$=\mathbf{2 3 r}$

Magnoliaceae Juss., DC.

Melesherbiaceae Don.

328

198

Malpighiaceae Juss.

Malvaceae Juss., Ktl.

Manettieae Cham. et

Schl.

Maquineae Mart.

Marantaceae Ldl.

Marattiaceae Kaulf.

Marcgraviaceae Juss.

286

296

Marchantiaceae Nees, Endl. 18

Narsileaceae $\mathbf{R B r}$.

176

$<2 \pm 1$

$<76$

$=2 \gamma$

305

Melampyraceae Rich. < 129

Melanthaceae $\mathbf{R B r} . \quad=\quad \boldsymbol{1}$

Melastomaceae Juss., Don. 236

Meliaceae Juss,, DG. 306

Memecyleae DC.

2335

Menispermaceae Juss, DC.32

Menyantheae Mart.

155

Mimoseae RBr.

223

Minuarticae Mart.

< 259

Monimiaceae Juss.

$<93$

Monimieae RBr.

93

MoNoch LAMYDEAE DC. Cl. V. MonocotyledoneaE Juss.,

DC. Cl. III et IV.

Monotropeare Nutt.
Moreae Endl., Mart. $\quad<91$

Moringeae BBr., Bartl. 224

Mucedineae Brongn.

Mucedines Nees.

$<2$

Multisiliquosae Batsch.,

Pb. O. 48.

Musaceae Juss., DC.

75

Mhusci Linn., Juss, $=C l$. $I I$.

MUscosaE Pb. Cl. II.

Mutisieae Cass.

Mutisiaceae Lessg. $\quad\}<186$

Nycetes Spr. $=\mathbf{C l} \mathbf{1}$.

Myelomycetes Nees.

Myoporineae $\mathbf{R B r}$.

Myricaceae Rich., Bartl. 83

Myresticeae RBr.

117

Myrobalaneae Juss.

110

Myrsineae RBr.

$=12 \gamma$

Myrtaceae Juss., RBr.

Myrtineae DC.

232

$=232$

Najadeae Rich.

32

Najades Juss.

$=\mathbf{3 1}$

Narcissi Juss.

et sqq.

Nassauriaceae Lessg. ?

Nassanvieae Cass. $\}<\mathbf{1 8 6}$

Nelumboneae DC., Bartl. 320

Nepentheae Ldl.

120

Neuradeae DC.

228

Nhandirobeae A. St. Ilil. $<\mathbf{1 9 5}$

Nitrariaceae Ldl.

$<256$

Nolanaceae Ldl.

145

Nopaleae DC.

$=\mathbf{2 4 5}$

Nostochinae Ag.

Nyctagineae Juss.

10

Nymphaeaceae Salisb.,

l)C.

Nyssaceae Juss.

$>319$

109

Ochnaceae DC.

281

Olacmeae Mirb.

Oleineae Lk.

300

Oleraceae $\mathbf{\Lambda g}$., Pb. O. 19.

Onagrae Juss.

Onagrariae Juss.
Opercularieae Juss.

Fam

Operculatae Web. et M. o. 5.

Ophioglosseae RBr. 23

Ophiospermeae Vent. $=\mathbf{1 2 7}$

Ophioxyleae Mart.

163

Opuntiaceae Juss., Kth. 24.5

Orchideae Linn., Juss. 78

Orobancheae Vent.

130

Orontiaceae Bartl.

36

Osmundaceae $\mathbf{R B r}$.

$2+$

Osyrideae Juss.

Oxalideae DC.

309

Paederieae DC.

$?<175$

Paeoniaceae DC.

326

Palmae Linn., Juss. O. 12.

Pandaneae $\mathrm{RBr}$.

40)

Pangieae Bl, Mart. $<26 \%$

Papaveraceae Juss., DC. 319

Papayaceae Mart.

196

Papilionaceae Linn., RBr. 221

Parideae Mart.

$<6 t$

Parkeriaceae Ilook. $<26$

Paronychieae St. Hill,

DC.

258

Passifloreae Juss.

197

Patmaceae Schultz. $<119$

Patrisiaceae Mart. $<\mathbf{2 ( 5 5 )}$

Pedalineae R3r. $=\mathbf{1 3 2}$

Pediculares Juss. $\quad<129$

Penaeaceae RBr., Kth. $11 t$

Peponiferae Pb. O. 33.

Perdicieae Spr.

186

Persanatae Adans., $\mathrm{Pb}$.

O. 23.

Petiveriaceae Ag., Lk. $<104$

Phalloideae Fr.

$<3$

Philadelpheac Don.

$2+7$

Philydreae RBr.

Phytelephanteae Mart. 41

Phytolacceae RBr., Bartl. 104

Picrochyleae $\mathbf{P b} .0 .2 \gamma$.

Pilomycetes Mart.

$<5$

Piperaceae IRiclı.

10 ?

Piperinae Bartl., Pb. O. 18 


\begin{tabular}{|c|c|c|}
\hline Pistacince Mart. & Restiaceae $\mathbf{R B r}$. & Saxifrageae DC. \\
\hline Pistiaceae Rich. & Restioneae Bartl. & Scaevoleae Ldl. \\
\hline Pittosporeae $\mathrm{RBr}$. & Rhamneac Juss., DC. & Schismatopterides $W .=2 \pm, 25$ \\
\hline Plantagineae Juss. & Rhinanthaceae Juss., & Schizaeaceae Mart. $\quad<\mathbf{2 t}$ \\
\hline Plataneae Lestib. $\quad 92$ & DC. & Schizandraceae Bl. \\
\hline Pleurospermae $\mathrm{Pb} .0 .38$. & Rhinantheae Vent. & et $\mathbf{3 3 0}$ \\
\hline Plumbagineae Juss., Vent. 125 & Rhizantheae BI. & Scitamineae Linu., Spr. $\quad$ 6 \\
\hline Podophylleae DC., LdI. 322 & Rhizoboleae DC. 288 & Scitamineae Vent. $=\mathbf{7 5}$ \\
\hline Podostemeae Lk. & Rhizocarpae Batsch. $=28 \mathrm{ct} 29$ & Sclerantheae Lk. \\
\hline $\begin{array}{l}\text { Polemoniaceae Juss., } \\
\text { Vent. }\end{array}$ & $\begin{array}{l}\text { Rhizophoreae } \mathrm{RBr} \text {. } \\
\text { Rhizopterides Mart. }\end{array}$ & $\begin{array}{l}\text { Scrophulariaceae Juss., } \\
\text { IRBr. }\end{array}$ \\
\hline Polemonieae Don. $\quad 153$ & Rhizospermae DC. $=28,29$ & Scrophularinae Auct. $<129$ \\
\hline Polycarpaeaceae DC. & Rhodoraceae Vent. $\quad 168$ & Sebesteneae Vent. $>1 \pm \gamma$ \\
\hline Polygaleae Juss. & Rhoeadeae Linn., Batsch. $>\mathbf{3 1 \gamma}$ & Selagineae Juss. \\
\hline Polygoneae Juss. & Ribesiae A. Rich. $\quad=\mathbf{2 t 4}$ & Sempervivae Juss. \\
\hline Polypodiaceae RBr. & Ricciaceae Nees., Endl. $<16$ & Senecioneae Cass. \\
\hline Pomaceae Juss. & Rigidae Batsch., Pb. O. 29. & Senecionideae Lessg. \\
\hline Pontederiaceae A. Rich. $\quad 63$ & Riviniaceae Ag. $\quad \succ 104$ & et 185 \\
\hline Poropterides Willd. $=\mathbf{2 \gamma}$ & Rosaceae Tourn., Pb. O.37. & Sesameae DC. \\
\hline Portulaceae Juss., DC., & Rosaceae Bartl. $\quad<\mathbf{2 3 0}$ & Sileneae DC. \\
\hline Bartl. & Roseae DC. $\quad<230$ & Simarubaceae Rich., DC. 280 \\
\hline Potalieae Mart. & Roxburghinceae Wall. $<$ ? 64 & Smeathmannieae Mart. $\quad 2 \pm 0$ \\
\hline Potameae Juss. $\quad=32$ & Rubiaceae Juss., DC. $<0.29$ & Smilaceae $\mathbf{R B r} . \quad<6 \pm$ \\
\hline Potamogetoneae Mart. $<32$ & Rutaceae Juss., DC. > 2 ร & Solaneae Juss., Bartl. $\quad 149$ \\
\hline Potamophilae Rich. $\quad=32$ & Rutariae $\mathrm{Pb} .0 .42$. & Spadicinae $\mathbf{A g} ., \mathrm{Pb} .0 .0$ \\
\hline Potentilleae Juss. & Ruteae Ad.Juss. & Sparganioideae Lk. $\quad<39$ \\
\hline Primulaceae Vent. & & Spermacoceae Cham. et \\
\hline Proteaceae Juss. & Sabalince Mart. & Schi. $\quad<185$ \\
\hline Protophyta Pb. Cl. I. & Salicariaceae Juss. & Sphagnaceae Nees. \\
\hline Psychotriaceae Cham. et & Salicinae Rich., Bartl. & Spigeliaceae Mart. \\
\hline Schl. $\quad<\mathbf{1 8 5}$ & Salviniaceae Bartl. & Spiraeaceae DC. \\
\hline Pteleaceae Kth. $\quad<2$ (89 & Salvinieae Mirb. & Spondiaceae Kth. $\quad 216$ \\
\hline Pyrenaceae Vent., Pb. O. $2 \sharp$. & Sambucinae Batsch. & Squamiflorae $\mathbf{P b}$. Lehrb. = \\
\hline Pyrenomycetes Fr. 4 & Samolinae Raf. & $0.15-18$ \\
\hline Pyrolaceae Ldl. & Samydeae Vent. & Stackhousieae $\mathrm{RBr}$. \\
\hline Pyroleae Nutt. & Sanguisorbaceae $\mathrm{Pb}$. & Staphyleaceae DC., Bartl. 211 \\
\hline & Sanguisorbeae DC. & Stellatae Linn., DC. \\
\hline Radiatae Linn. & Santalaceae RBr. & Sterculiaceae Kth. \\
\hline Rafflesiaceae $\mathbf{R B r}$. & Sapindaceae Juss. & Stilagineae Ag. \\
\hline Ramalineae Ag. $\quad$ D & Sapoteae Juss. $\quad 165$ & Stilbineae Kth. \\
\hline Ranunculaceae Juss., DC. 325 & Sarment aceœ Vt., Pb. 0.46. & Stratzoteae Lk. \\
\hline Rapunculeae $\mathrm{Pb} .0 .32$ & Sarmentaceae Vent. $=\mathbf{3 0 8}$ & Strobilaceae Rchb. \\
\hline Rauwolfiec DC. $\quad=163$ & Sarraceniaceae Lapyl. $\quad 318$ & Strychneae Pet.-Th., \\
\hline Reaumuricae Ehrbg. & Saurureae Rich. & DC. \\
\hline Resedaceae DC. $\quad 2 \gamma 1$ & Sauvagesiaceae DC., Mart. 267 & Strychneae Mart. \\
\hline Resinara Batsch,, Pb. O. 35. & Saxifragaceae DC. $>248$ & Stylardrœ Pb. 0. 21. \\
\hline
\end{tabular}




\begin{tabular}{|c|c|c|}
\hline Stylidieae RBr., & Thymeleae Juss. & Valerianeae DC. \\
\hline tyraceae Rich. & Tiliacene Juss. & Vallisneriaceae Lk. \\
\hline tyracinae Bartl. & Tithymaloideae Vent. & Valvatae $\mathrm{Pb} . \quad=\mathbf{1 7}-\mathbf{1 9}$ \\
\hline $\begin{array}{l}\text { ucculentae Linn., } \\
\text { Pb. O. } 39 .\end{array}$ & $\begin{array}{l}\text { Tremandrece } \mathbf{~} \mathrm{Br} \text {. } \\
\text { Tremellinae } \mathbf{F r} \text {. }\end{array}$ & $\begin{array}{l}\text { VASCULARES DC. Cl. IIIet sqq. } \\
\text { Veratrene Salisb., Ag. }=\boldsymbol{7 1}\end{array}$ \\
\hline umachineae DC. & Trichiaceae Fr. & Verbenaceae Juss. \\
\hline wartzieae DC., & Linn., Bartl. J & Vernoniacene Lessg. \\
\hline ymploceae Juss. & Trigoniaceae Mlart. $<\mathbf{2 8 t}$ & 187 \\
\hline $\begin{array}{l}\text { ynanthereae Rich., } \\
\mathrm{Pb} .0 .31 .\end{array}$ & $\begin{array}{l}\text { Trihilatae Linn., Pb. } 0.43 . \\
\text { Tripetaloideae Linn., Pb. } \\
\quad \text { O. } 11 .\end{array}$ & 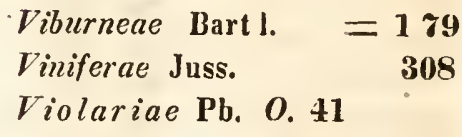 \\
\hline acceae Presl. & leae Juss, & ae DC. $\quad=\mathbf{2 6 0}$ \\
\hline amariscineae Desr. & ceae Fr. & Viticeae Juss. \\
\hline Targioniaceae $\mathrm{N}$ & Talipaceae DC. $\quad<67$ & iaceae St. Hil. \\
\hline easch. & ceae Kth., DC. 242 & \\
\hline Terebinthaceae Juss., Pb & Tussilagineac Cass. $\quad<185$ & Wallichieae DC. \\
\hline et $s$ & Typhaceae Juss. & Wintereae $\mathbf{R B r}$. \\
\hline$E R$ & Ulma & Xanthoxyleae Nees et \\
\hline .299 & ze $\mathrm{Ag}$ & Mare. \\
\hline 299 & Umbelliferae Juss. & Xeranthemeae Cass. \\
\hline $\begin{array}{l}\text { ze Pb. O. } 2 \\
\text { IAE Pb. Cl. }\end{array}$ & $\begin{array}{l}\text { Umbraculariae Batsch., } \\
\mathrm{Pb} \text {. O. 3t. }\end{array}$ & $\begin{array}{l}\text { Xylomyci Auct. } \\
\text { Xyrideae Ag. }\end{array}$ \\
\hline$H A L$ & Urtic & \\
\hline hala & Urti & uss. $=\mathbf{2 7 9}$ \\
\hline$<\mathbf{2 9 9}$ & Utric & $=76$ \\
\hline hylacopterides $\mathbf{P b}$. 0.8 . & Vaccinieae $\mathrm{D}$ & Zygophylleae RBr. \\
\hline
\end{tabular}




\section{Register}

\section{der Pfanzengattungen.}

Abatia R. et Pav.
Abelia RBr.
Abelicia Sm.
Abies DC.
Abildgaardia Vahl.
Ablania Aubl.
Abolboda H.B. K.
Abroma L. fil.
Abronia Juss.
Abrotanella Cass.
Abrus L. "
Abnta Aubl.
Abutilon Mch.
Abutua Lour.
Acacia Neck.
Acaena Vahl.
Acajuba Gaertn.
Acalyplua L.

Fam.

265

178

88

80

43

293

48

294

121 Acianthus RBr.

185 A cicarpa Radd.

221 Acicarplo Juss.

324 Acidolontium Schurgr.

296 Acidoton Su.

91 Acilcpis Don.

223 Acinaria Targ.

230 Acinos Vocnch.

219 Acinula $F r$.

96 Acioa Aubl.

Acanthephippium Bl. 78

Acanthodium Del. $\mathbf{1 8 1}$

Acanthoglossum $\mathrm{Bl}$. 78

Acantholepia Lessg. 181

Acanthoploora Lmrx. 15

Acanthophyllum

Neyer.

Acantophyton Less.

Acanthospermum

Schrk.

is canthus $L$.

Acarna $W^{\prime}$.

Acer $L$.

Aceranthus Morr.

Aceras $R B r$.

Accrates Ell.

Aceratium DC.

Achania Sin.

Acharitheriun Btff. et $F$.

Achetaria Cham.

Acliillea $\mathrm{L}$.

Aclimenes Iahl.

Achiton Corda.

Aehlya Nees.

Achlys DC.

Achnanthes Bory.

Achnatherum $P . B$.

Achnodonton P. B.

Achras $L$.
Acisanthera ${ }^{P} . B r$.

Aclalinu $L R$.

Acladoden R. ct Pav. ? 289

Aclisia E. Mcy.

Acmalenia Btl. et

262 Wdl.

188

Aemella Rich.

Acmena $D C$.

187 A cnida $L$.

131 Acolium Fée.

184 Aconitum $L$.

285 Acontias Schott.

323 Acaris $L$.

i8 Acosmus $D i s e$.

160 Aconroa $A u b l$.

292 Acratherum Lk.

296 Acremonium Lk.

Acridocarpus Giuil.

185 et $\boldsymbol{P}$ cr.

? 129 Acrinpsis Bl.

187

2135

288

187

Acrocomia Mart.

Acroglochin Schrad. 103

Acrolepis Schrad.

Acronia Pres?.

Acrnodia $B l$.

Acronychia $\mathrm{Bl}$.

Acropera Ldl.

Acropteris $L k$.

\section{Aciotis Dorr.}

Fam.

78

105 Acrosporium Nees

185 Acrostichum $L$.

221

188

188

142

225

78

42

18:3

21

96

185

15

142

3 Ictind

anodontium

225 Schuagr.

236 Actinolepis $D C$.

238 Actinomeris Nutt.

2 Actinonema Pers.

Actinoplyyllum $\boldsymbol{R}$. et

49 Pav.

Actinospora Turcz.

Aetinotliyrium $K u$.

232 Actinotus Labill.

103 Adambea Lam.

6 Adamia Wall.

325 Adamsia $W$.

36 Adansonia $L$.

37 Allelin $L$.

286 Alelobotrys $D C$.

221 Adenandra $W$.

12. Adenanthera $L$.

2 Adeninthes Labill.

Adenaria $I . B . K$.

286 Adenarium Raf.

88 Adenium Ehrb.

Acrocephalus Benth. 142 Adenocarpus $D C$.

62 Adenocaulon Hook.
43 Adenodus Lour.

78 Arienolepis Lessg.

? 292 Adenoncos $B l$.

278 Adenophora Fisch.

58 Adenophorus Gaudich. 2

26 Adenophyllum Pers. 18r
Fam. Fam.

3 Adenorhopium Pohl. 96

2 Adenosma $R B r$. 131

26 Adenostemma Forst. 185

Adenostemon Pers. ? 116

? 327 Adenostyles Cass. 185

172 Adenostylis $\mathrm{Bl}$. 78

42 Adesmia $D C$. 221

?292 Adina Salisb. 17

326 A.dleria Neck. 222

4 Adlumia Raf. 316

187 Adonis $L$. 325

18. Adoxa $L$. 201

51 Ailrastea $D C$. 327

42 Adriana Gaudich. 96

2 Adupla Bosc. 43

3 Aechmea R. et Pav. 56

Accidium Pcrs. 1

21 Acgerita Pers. 3

187 Aegialina Schult. 42

187 Aegialitis Triu. 42

4 Aegialitis $R B r$. 125

Aegiceras Gaertn. 127

201 Aegilops $L$. 42

326 Acrinetia Roxb. 130

21 Aegipliila $L$. $\quad 138$

4 Aegle Corr. 301

200 Aegopodium $L$ : $\quad 200$

238 Aconopogon P.B. 42

522 Aegopricon L. fil. - 96

(i6) Acluropus Trin. 42

295 Aeolanthus Mart, 142

96 Aëranthus Ldl. · 78

230 Aérides Lour. 78

258 Aërnbion $S p r . \quad$ is

223 Ä̈rophyton Eschw. 2

113 Aërophyton Nieyen. $\quad 10$

238 Aërva Forsk. $\quad 105$

261 Acschynantlıns Jack. 134

161 Aescliyumene L. 221

221 Aescnlus $L$. 28 \%

185 Aethalium $L k$. 3

185 Actlieilema $R B r$. 131

292 Aetheorrhiza Cass. 188

187 Actheria $\mathrm{Bl} . \quad \mathbf{3 8}$

78 Aetluonema $R B r$. 314

194 Acthionia Don. 188

Aethuea $L$. $\quad 200$

Aetia Ad. 


\begin{tabular}{|c|c|c|c|c|c|c|c|}
\hline & & & & & & & Fam \\
\hline fzelia $G m$. & 129 & Ajovea Aubl. & 116 & & 66 & Ameletia $D C$. & \\
\hline Afzelia $S m$ & 222 & Ajuga $L$. & 1.12 & Aloèxylon Lour. & 222 & Amellus $L$ & 187 \\
\hline Agapanthns Her. & 67 & Akeesia Tuss. & 289 & Alomia $H . B . K$ & 185 & Amerimnum $P . E r$. & 221 \\
\hline gardhia Gray. & 12 & Alamania Llav. & 78 & Alonsoa $R$. et Pav. ? 1 & 148 & Amethystea $L$. & \\
\hline gardhia Cabr. & 14 & Alandina Ncck. & 221 & (129) & & Amherstia Wall. & \\
\hline gardhia $S p r$ & $? 291$ & Alangium Lam. & 206 & Alopecurus $L$. & 42 & Amicia $I I . B . K$. & \\
\hline garicus $L$. & 5 & Alarçonia $D C$. & 187 & Alophochloa Rchb. & 42 & Amirola Pers. & \\
\hline Agariata $D C$. & 187 & Grev. & 15 & Aloysia Ort. & 138 & Ammannia $L$. & \\
\hline Agarum Bory. & 15 & inia $S p r$. & 185 & Alphitomorpha Wallr. & & Ammi $L$ & \\
\hline Agastachys $R B r$. & 113 & Presl. & 43 & Alpinia $L$. & 86 & obium $R B r$ & 155 \\
\hline gasyllis $I l o f f m$. & 200 & Alibu & 66 & Alseis Schott. & 176 & ocharis Nlerb. & \\
\hline a Cass. & 187 & Alce & 296 & Alseodapline Nees. & 116 & dendron Fisch. & \\
\hline Agatlelepis Chois. & 140 & illa $L$ & 230 & Alsidium $\mathrm{Ag}$ & 15 & ophila IIost. & \\
\hline gathis Salisb. & 80 & Alchornea Sw. & 96 & Alsine $L$ & 261 & yrsine Pursh. & \\
\hline gathisanthes $\mathrm{Bl}$. & 110 & Alcicornium Gaudich. & - 26 & ia Pet. - Th. & 266 & $\operatorname{lm} L$ & \\
\hline Agathoplyyllum Comm & $m .116$ & Alcina Pers. & 187 & Alsomitra $B l$; & 195 & nia Nestl. & \\
\hline Agathosma $\mathrm{W}^{\prime}$ & 278 & $D C$ & 187 & Alsophila $R B r$. & 26 & ora $R x b$ & \\
\hline sus $D_{0}$. & 188 & Aldaina Llav. & 187 & $R B r$ & 161 & $L$ & 321 \\
\hline Rheed. & 221 & - et Pao. & 149 & Alstonia Mut. & 168 & ophallus $B l$. & \\
\hline$L$ & 56 & da Mont. & 268 & Alstroemeria $L$ & 69 & Ampacus $R p h$ & \\
\hline gdestis Moc.ct Siss. & s. 321 & Ach. & & Altensteinia H. B. K. & & Aimpelodesmos $L k$. & \\
\hline geratum $L$ & 185 & lophus M.B. & 129 & era Forsk. 1 & 105 & sis Michx. & \\
\hline ers. & 74 & Alec & 130 & ia Necs. & 1 & Ad. Juss. & \\
\hline glaja Lour. & 306 & Alec & 289 & Alth & 296 & phis $H . B . K$. & \\
\hline glaomorpha Schott. & 26 & Laroch. & 200 & Alt & 32 & Ell. & 291 \\
\hline a Schott. & 36 & R. Br. & 44 & $-T h$ & 294 & a $D C$ & \\
\hline Raf. & 188 & Alet & 66 & Alti & 87 & m $N_{c e s .}$ & \\
\hline ais $L k$. & fio & Ale & 5 & & 123 & $F r$ & \\
\hline graulos $P . B$. & 42 & $L k$ & 2 & eck. & 221 & ium Schott & \\
\hline ins Mart. & 185 & Forst. & 96 & Knz. & 2 & & \\
\hline$L$ & 230 & ndron Reinw. & 294 & & 14 & H. B. K. & . 133 \\
\hline dron Ilaw. & 66 & HI. B. K. & 62 & im $L k$. & $? 2$ & lart. & \\
\hline Juss. & 187 & iss. & 181 & & 163 & $\Delta \mathrm{m}$ & \\
\hline Gartn. & 42 & urn. & 221 & & 210 & on $R B r$. & \\
\hline ma $L$ & 262 & A. Rich. & 176 & 1 & 176 & $D C$ & 187 \\
\hline a Radd. & 42 & ess. & 187 & & 5 & Im $L k$ & \\
\hline Agro & 42 & Neck. & 295 & & 96 & dll. & 221 \\
\hline hyllum $B l$. & 78 & Alis & 51 & Am: & 15 & Desv. & 9. 26 \\
\hline$L$ & 96 & his Pct.Th. & 78 & $B l$. & 75 & $t .-T h$ & \\
\hline$F r$ & 5 & us Cass.? & 185 & & 105 & An & \\
\hline spr. & 129 & $L$ & 161 & & 222 & & 161 \\
\hline cour. & ? 178 & $R B r$ & 26 & & 69 & \& $L$ & 226 \\
\hline Aikinia Wall. & 42 & our. & 195 & & 138 & s $L ., K t h$ & 213 \\
\hline Desf. & 269 & Alli & 314 & & 62 & Anabaena $A d$. Juss. & \\
\hline eca $D C$ & 187 & & 124 & & 84 & Bory. & \\
\hline nes $W$ & $? 61$ & r. & 66 & alis. & 21 & & 103 \\
\hline ra & 42 & 18 H.B. K. & 187 & $8 B l$. & 88 & pta Roehl. & 21 \\
\hline a $L k$ & 42 & $18 L$ & 289 & $D C$ & 87 & seros $A d$ & 258 \\
\hline iropsis Dcsu. & 42 & im $L k$. & 3 & Ambor & 93 & Anacampseros Sims. & $25 \%$ \\
\hline itonia Forst. & 16 & Bernh. & 26 & Ambraria Crus. & 75 & Anacamptis Rich. & 78 \\
\hline L. fil. & $? 306$ & Alloteropeis Presl. & 42 & Ambrosia Cav. $\quad 290$ (18) & & Anacamptodon Brid. & 21 \\
\hline $\operatorname{zoon} L$. & 256 & Almeidea St. IIil. & 288 & Ambrosinia $L . \quad$ ? & 36 & $\operatorname{dium} L$ & 219 \\
\hline jax Salisb. & $69^{i}$ & Alnus $W$. & $84^{\prime}$ & Amelanchier Med. & $31^{1}$ & Anacharis Rich. & \\
\hline
\end{tabular}


Anacis Schrk.

Anactis Cass.

Anacyclus $L$.

Anadenia $R B r$.

Anagallis $L$.

Anaglypha $D C$.

Anagyris $L$.

Anaitis $D C$.

Anamirta Colebr.

Ananassa Lindl.

Anandria Siegesb.

Anantliopus Raf.

Anarrhinum Desf.

Anarthria $R B r$.

Anarthrosyne E.M.

Anasser $\mathbf{J}$ uss.

Anastatica $L$.

Anatherum $P$. $B$.

Anatropa Ehrbg.

Anavinga Lam.

Anaxagorea St. Ilil.

Anaxeton Gaertn.

Anaxetum Schott.

Anchietea St. Hil.

Anchionium $D C$.

Anchusa $\boldsymbol{L}$.

Ancistrocarpus II.B.K.105

Ancistrocladus Wall. ? 286

Ancistrum Forst.

Ancylanthus Desf.

Anda $P$ is.

Andersonia $R B r$.

Andersonia Roxb.

Andiantum $L$.

Andira Lam.

Andrachne $L$.

Andraspis Duby.

Andreaen Lhrh.

Andreoskia $D C$.

Andrewsia $V$ cnt.

Andrewsia $S p r$.

Andrieuxia $D C$.

Androcera Nutt.

Androcymbium $\boldsymbol{W}$.

Andromachia $H . B$.

Aildromeda $L$.

Androphylax $W$ endl.

Andropogon $\boldsymbol{L}$.

Androsace $L$.

Androsaemum All.

Andryala $L$.

Andrzeiowskia $R$ chb.

Aneilema $R B r$.

221

158

314

26

266

314

286

172

306

71

187

169

321

303

188
Fam.

187

181 Ancmagrostis Trin.

187 Anemarrhena Bung.

113 Anemone $L$.

126 Anethum L.

187 Ancura Dumort.

221 Aneuriscus Presl.

187 Angelica $L$.

324 Angelonia II. B.

56 Angiopteris Hoffm.

186 Angioridium Grcv.

49 Angolamia Scop.

129 Angophora Cav.

42 Anguloa $R$. et $P a v$.

276 Anguria $L$.

239 Ania Lill.

330 Aniba Aubl.

185 Anigozanthos Labill.

143

230

175

96

26

221

36 Aumeslea Wall.

126 Annesorhiza Cham. ct

20 Schl.

314 Anoda Cav.

139 Anoectangiun Iledw.

156 Anoectochilus $\mathrm{Bl}$.

187 Anoegosanthus Rchb.

118 Anoma Lour.

42

126

314

19) Antennaria $L k$.

Anomatheca Ker.

Anomodon Hook.

Anona $L$.

Anopterus Labill.

Anosporum Nces.

Anotis $D C$.

Anredera Juss.
Fam

21 Antennaria $R B r$.

Fan.

42 Antennularia Rchb.

185

Anthemis $L$.

3

(ii)

325

200

19

302

200

129

27

3

206

232

Anthenantia P. B.

187

Anthephora Schreb.

42

42

Anthericlis Raf.

Anthericum $L$.

Antherura Lour.

Antherylium Rohr.

Anthestiria $L$.

Anthina Fr.

Anthobolus $R B r$.

Anthocercis Labill.

Authoceros Micle.

Anthochloa Nices.

Anthochortus Nees.

Anthocleista Afzel.

78

66

175

237

42

2

108

129

17

42

45

159)

78

195

\%8

116

32

103

185

142

188

148

142

96

78

42

Anthodendron $\boldsymbol{R} c h b$.

Anthodon R. et Pav.

Anthodus Mart.

168

281

Anthogonium IVall.

Antholoma Labill. ? 305

Antholyza $L$.

Anthonota $P . B$.

$28 !$

78

24

74

Anthopogon Nutt.

222

Anthostema Ad. Juss. 96

Anthotium $R B r$.

190

Anthoxanthum $L$.

42

Anthriscus Hoffm.

200

Anthrocarpum Meyer.

Anthurium Schott.

Anthyllis L.

Antiaria Lesch.

200 Antichorns L. fil.

3 Antiderma $L$.

?299

Antirhoea Comm.

Antirrhin um $L$.

200

296

Antitragus Gaertn.

Antitrielia Brid.

Antomarchia Coll.

78

72

222

286

It

21

330

219

43

Anomostephium DC. 187

176

103

Antrophyum Kaulf.

Anvilbea $D C$.

Anychia Michx.

Aopla Ladl.

Aotus Sm.

Apalatoa Aubl.

Apalus DC.

Apargia $L$.

A patelia $D C$.

Apaturia $L d l l$.

03 A phanamixis

3 Aphance $L$.

175

129

42

21

278

26

155

258

78

221

222

187

188

299

Fam.

289

Apùania Bl.

142

Aphranostemma St.Hil. 325

Aphanostephus DC. $\quad 187$

Aphelandra $R B r . \quad 131$

$\Delta$ phciexis $D_{0}$. $\quad 185$

Aphelia $R B r$. $\quad 44$

Aphragmus Andr. $\quad \mathbf{3 1 4}$

Aphyllanthes $B$. ? 48

Aphyllocarpa Cav. 21

Aphyllocaulon Lag. 187

Apliyteia $L . \quad 113$

A picra W. $\quad 60$

A pios Unch. 221

Apiosporinm Knz. 3

Apistal $B l . \quad 78$

Apium L., Hoffm. 200

Aplectrum Nutt. $\quad 78$

Aplodon $R B r$. 21

Aplopappus Cass. $18 \mathrm{~T}$

Aplophyllum Ad. Juss. 270

Aplotella Kü̈t. $\quad \mathbf{1 1}$

Apluda $L$.

Apocynum $L . \quad 161$

Apodanthes Poit. ? - 119

Apodznomene E.H. 221

Apoma Endl. 3

Aponoget on Thunb. 99

Aporetica Forst. $\quad 289$

6 Aporum Bl. 78

36 A poseris Neck. 188

221 A postasia $\mathrm{Bl}$. 78

91 Apoterinm $\mathrm{Bl}$. 302

298 Appendicula $\mathrm{Bl}$. $\quad \mathbf{8}$

95 Appendicularia $D C . \quad 230$

is Arauja Brot. $\quad 160$

293 Arbutus $L$. $\quad 169$

306 Areeuthobium M. B. 203

230 Archangelica Hoffm. 200 
Archemora $D C$.

Archidium Brid.

Fam.

200

21

Archytaea Mart.etZuec. 299

Arctium Lam.

Arctogeron $D C$.

Arctopus $L$.

Arctostaphylos Ad.

Arctotheca Wendl.

Arctotis $L$.

Arcyphyllum Eill.

Arcyria Hill.

Ardisia $S w$.

Arduina $L$.

Areca $L$.

Arerma $\mathrm{Fr}_{2}$.

Aremonia Ncek.

Arenaria $L$.

Areng Labilı.

Arethusa Gron.

Argyrochaeta Cav.

Aretin $L$.

Argania Schousb.

Argemonc $L$.

Argolasia Juss.

Argophyllum Forst.

A rgostemma W'all.

Argylia $D o n$.

Argylium Wallr.

$\Lambda$ rgyrcia Lour.

Argyrocome Gaertn.

Argyroxiphium $D C$.

Argythamnia $P . B r$.

Ari⿵aema Mart.

Arisarum Tourn.

Aristea Ait.

Aristella Trin.

Aristida $L$.

Aristolochia $L$,

Armania Bert.

Armeniaca Tourn.

Arueria $\boldsymbol{U}$.

I87 Artabotrys $R B r$.

200 Artedia $L$.

169 Artemisia $L$.

187 Arthonia Ach.

187 Arthratherum. P. B.

221 Arthraxon Pers.

3 Arthrinium Kinz.

126 Arthrolobium Desv.

162 Arthrolobus Stev.

61 Arthronaria Fr.

1 Arthrophyllum $B l$.

230 Arthropodium $R B_{r}$.

62. Arthrostcmma Pav.

78

187

126

165

317

i2

170

176

133

3

150

185

187

96

36

36

42 Asclepias $L$.

42 Ascobolus Pers.

121 Ascophora Tod.

187 Ascophora Fr.

226 Ascoxyta Lib.

125 Ascra Schoti.

Armodorum Kuhl.et H. 78

Arnica $L$.

Arnoldia Cass.

Arnoldia $B l$.

Arnopogon $W$.

Arnoseris Gaertn.

Arnottia A. Rich.

Aromadenitron $\mathrm{Bl}$.

Arongana Pers.

Aronia Pers.

Arpophyllum Llav.

Arracacha Bancr.

Ascroé Labill.

188 Ascyrum $L$.

251 Asimina Alans.

188

188

Aspalathus $L$.

Asparagus $L$.

Aspasia $L d l$.

303

2211

o Asergillis Mich.

200 Asperugo $L$.
261 Arthrostacliya $L i$.

74 Ascium Schrb.

Fam.

185 Asperula $L$.

Fam.

174

66

66

302 Asphodelus $L$.

2315 Aspicarpa Rich.

330 Aspidistra $K e r$.

200 Aspidium Sw.

286.

? 64

26

185 Aspidosperma Mart. I61

6 Aspilia Pet. - Th.

187

26

42

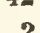

2 Assonia Cav.

221 Astartea $D C$.

314 Astemma Less.

6 Astelia Bks. et Sol.

201 Astelma $R B r$.

66 Astephanus $R B r$.

42 Aster $L$.

236

Asteranthus Desf.

Asterisca Meyer.

79)

91

278

36

Asteriscium Cham. et Scht.

Asteriscus Much.

42

294

232

185

? 46

185

160

188

199

6

200

Asterocephalus Spr.

187

182

43

22 Asterochate Nces.

i8 Astcrolinon $L^{k}$.

42 Asteroma $D C$.

42 Asteromaea $B l$.

42

Asterophora Dittm.

? 138 Asteropsis Less.

58

121 Asterosperma Less.

185 Asterosporium $K n$ z.

126

4

I87

Aubletia Gaertn.

187 Aubletia Pers.

187 Aubletia Schreb.

187 Aubrictia $A d$.

I Aucuba Thunb.

101 Astcrothecinm W'allr.

3 Asterothrix Cass.

305 Astianthus Don.

160 Astilbe Ham.

5 Astoma $D C$.

2 Astragalus $L$.

4 Astrantia $L$.

4 Astranthus Lour.

295 Astrapaca Lell.

3 Astrephia Dufr.

303

187 Aseimotrichnm Corda.

Astrocaryum $W$. Mcyer, 62

Astrodontium Schugr.

ชือ Astrolobium $D C$.

201 Astroloma $R B r$.

64 Astronia $\mathrm{Bl}$.

Aspegrenia Pöpp. et E. 78

is Aspcrococcua $2 \mathrm{mrr}$.
78

Astroniun Jacq.

Astrophea DC.

187 Astrothclium Fschu.

2 Astrotricha $D C$.

15 Astydamia $D C$.

110 Atacria Presl.
3

188

133

218

200

221

200

24

291

180

21

221

172

236

219

? 210

6

200

200

70
Fam.

188

301

? 315

42

78

200

18 อ

5

239

42

4)

36

187

40

127

?

3

? 187

325

106

300

21

103

148

62

279

209

232

258

293

314

202

112

12

208

156

176

$\begin{array}{ll}\text { Aulacia Lour. } & 301 \\ \text { Aulacinthus E. M. } & \mathbf{2 2 1}\end{array}$

$\begin{array}{ll}\text { Aulacia Lour. } & 301 \\ \text { Aulacinthus E. M. } & \mathbf{2 2 1}\end{array}$

Aulacomnion Schugr. 21

Aulacorhynchus $N$ ees.

Aulax Berg.

Aulaxanthus Eli.

113

Aulaxina Fée.

Aurelia Cass. 
Axinopus licem. et Sch. 42

Axonopus P.B.

Axyris $L$

Ayenia $L$.

Azadirachta Ad. Juss. 300

Azalea $L$.

Azara $\boldsymbol{R}$. ct $\boldsymbol{P a v}$.

A zima Lam.

Azolla Lam.

Azorella Gaudich.

Azosma Corda.

Azygites $\mathrm{Fr}$.

Babiana $\mathrm{Ker}$.

Bacazia R. et Pav.

Baccalaria Gray

Baecharis $L$.

Baconia $D C$.

Bacopa Aubl.

Bactridium $K n z$.

Bactris Jacq.

Bactyrilobium $\mathrm{W}$.

Badaria Gaertn.

Badiera DC.

Badula Juss.

Baea Comm.

Baeckea $L$.

Baenbotrys Forst.

Baeometra Salisb.

Baconyces Ach.

Bacothrion Nees.

Bagalatta Roxb.

Bagassa Aubl.

Bahia Lag.

Baillieria Cass.

Balanites Del.

Balanophora Forst.

Balanopteris Guertu.

Balantium Kaulf.

Balbisia $\boldsymbol{W}$.

Balbicia Cav.

\begin{tabular}{l|l}
276 & Batis P.Br. \\
tt. 42 & Batrachium DC.
\end{tabular}

74 Barclaya Wall.

186 Barkania Ekrbg.

15

185

175

126

2

62

222

110

272

122

129

232

? 126

\%1

8

43

324

91

187 Basilaea Lam.

155 Bassia All.

? 300 Bassia Koen.

Baldingera $\mathrm{Fl}$. Wett.

Balduina Nutt.

Balfouria $R B r$.

Ballota $L$.

Balsamia Vittad.

Balsamina Riv.

Balsamita Desf.

188

16

142

3 Batschia Vahl.

313 Batschia Thunb.

? 185 Battarea Pers.

Balsaunodendron Kth. 215 Baudinia Lesch.

Balsamoua rand.

23ร Bauera Kenn.

Balsamorhiza Hook, 187

Baltimora $L$.

Bambos Retz.

187 Baumea Gaud.
Fam.

42

42

205

262

12

280

113 Beanmontia Wall.

222 Bechium $D C$.

221 Beckeria Bernh.

42 Baumgartenia Spr.
? 72

314

221

21

319

32.

188

101

186

65

288

201

232

78

175

232

156

213

$\because 1$

129

221

.278

222

108

66

103

165

148

296

78

\section{6}

325

42

42

143

222

324

? 3

232

250

222

43 Berula Koch.

Beckmannia Host.

Beclardia A.Rich.

Beera $P$. $B$.

Bcesha Rheed.

Befaria Mut.

Begonia $L$.

Belangera Camb.

Belemcanda Mnch.

Belis Salisb.

Bcllardia AU.

Bellardia Schreb.

Bellendena $R B r$.

Bellevalia Lap.

Bellidiastrum Mich.

Bellinia $R$. et Schult.

Bellis $L$.

Bellium $L$.

Belvisia Desv.

Benincasa Savi.

Benthamia A. Rich,

Benzoin Nees.

Bequerela Brongn.

Berarlia Brongn.

Berberis $L$.

Berchemia Neck.

Berehtoldia Persl.

Bergenia Mnch.

Bergera lioen.

Bergia $L$.

Beringeria Neck.

Berkeleya Grev.

Berlheya Florh.

Berlandiera $D C$.

Bermudiana Gaertn.

Bernardia Houst.

Berrya Roxb.

Berteroa $D C$.

Bertholletia HI. B.

Bcrtiera Aubl.

Bertolonia $D C$.

Bertolonia Radd.

? 66 Berzelia Jart.
Fan.

36

160

263

232

av.

302

161

185

42

42

78

43

42

180

108

251

74

80

129

176

113

66

187

148

187

187

199

195

78

116

43

208

323

209

42

248

301

260

142

11

187

187

74

96

293

314

232

170

186

236

302

200

105

Berzelia Brongn.

Besleria $L$.

Bessera Schult.

Bessera Spr.

Beta $L$.

Betckea $D C$.

Betonica $L$.

Betula $L$.

Fan.

908

?. 135

143

255

103

180

$1+2$

Beureria Jacq. ? 14ti

Beurreria Ehr. $\$ 33$

Beyrichia cham. I20

Biaruin Schott.

36

Biaslia Vand.

19

Biasolettia Presl.

294

Bichenia Don.

186

Bicornella Ldl.

78

Biddulphia Gray.

11

Ridens $L$.

187

Biebersteinia Steph.? 270

Bifora Hoffm.

200

Biforis Spr.

200

Bifrenaria $L d l$.

78

Bigelovia Spr.

173

Bigelowia $D C$.

187

Bignonia $L$,

1333

Bikia Reinz.

176

Bilabrella $L d l$.

78

Billardiera Vahl. 


\section{- 5}

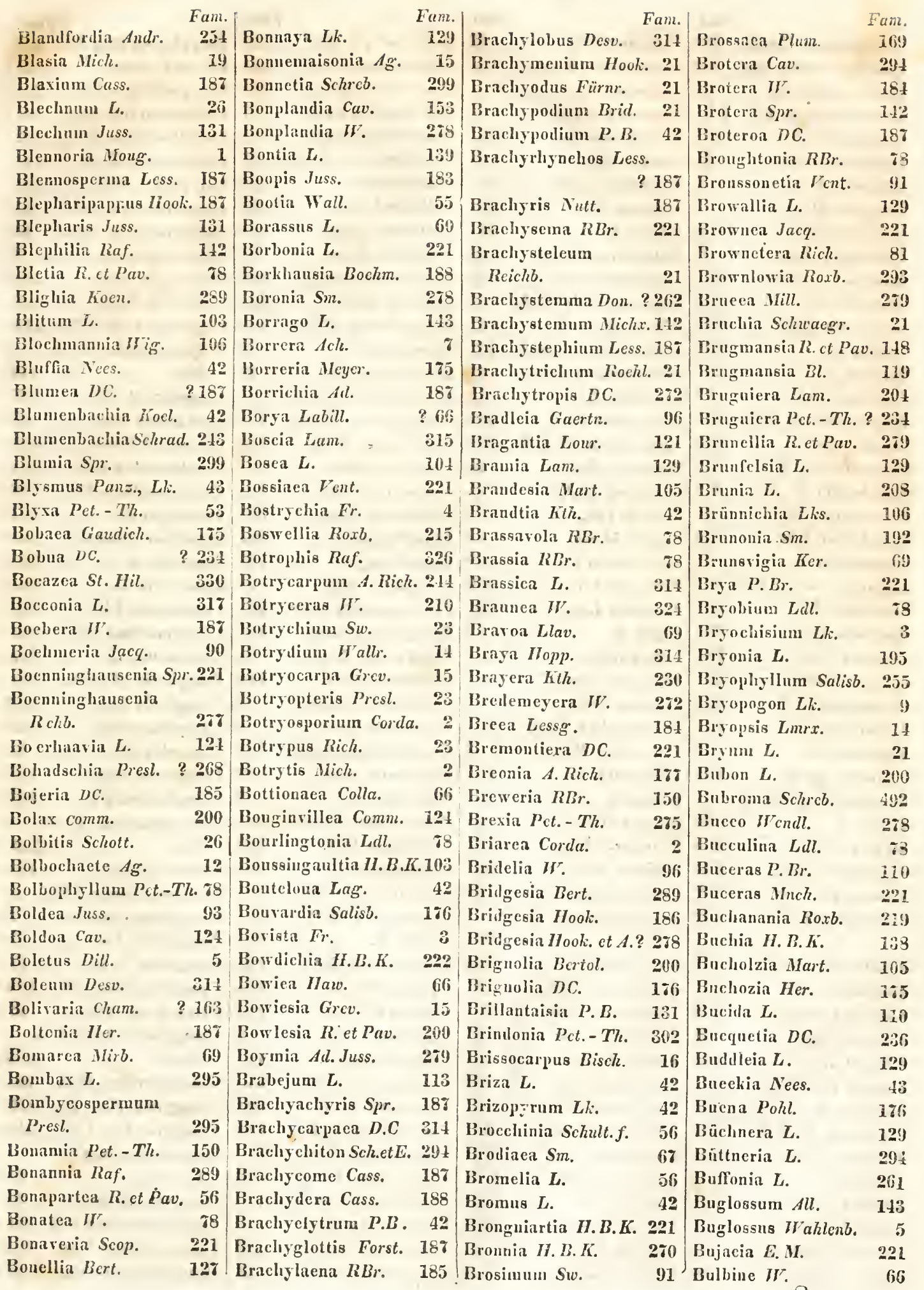




\begin{tabular}{|c|c|c|c|c|c|}
\hline ulbocapnos Bernh. & Fam. & Cadmus Bory. & $\begin{array}{r}a m . \\
12\end{array}$ & Callistachya Raf. & $\begin{array}{r}\text { Fant. } \\
\text { Calyplectus } R \text {. et Pav. 23t }\end{array}$ \\
\hline ulbocastanum Lag. & 200 & Caenopteris Berg. & 26 & Callistachys Vent. & Calypso Salisb. $\quad 78$ \\
\hline ulbocodium $L$ & 67 & Cacoma $L k$. & $\mathbf{1}$ & Callistemon $R B r$. & Calypso Pet. - Th. \\
\hline num $B l$. & 71 & urus $L k$. & 1 & Callistephus Cass. & Calyptocarpus Less. \\
\hline$D C$. & $18 \tilde{5}$ & esalpinia $L$ & 222 & Callisthene Mart. 291 & Calyptranthes Sw. \\
\hline is Stev. & 47 & aesia $R B r$ & 66 & Callithannion Lyngb. 12 & Calyptranthus $B t$. \\
\hline & 5 & Roxb. & 185 & Callitriche $L$. & Calyptrion Ging. \\
\hline & 1 & $D C$ & 221 & Callitris Vent. & Calyptrocarya Nces. \\
\hline$D C$ & 255 & & 314 & e Comm. & Calyptrostylis Nees. 43 \\
\hline Jungh. & 3 & ia $R B r$ & 78 & Callixene Lam. & Calyssosporium Corda. 2 \\
\hline & 211 & n Vent. & 36 & ma Mart. & Calystegia $R B r$. $\quad 150$ \\
\hline & 165 & rostis Reth. & 42 & Calluna Salisb. & Irix Labill. $\quad 232$ \\
\hline Rich. & 286 & $B$. & 42 & Calocephalus $R B r$. & iplex R. et Pav. 12y \\
\hline & 314 & a Mocnch. & 142 & Caloc & nia $R$. et \\
\hline & 200 & loè Rehb. & 42 & Calochilus $R B r$ & 124 \\
\hline lerb. & (6) & & 59 & Caloclsorthus Pursh. & Camarea St. Hit. \\
\hline in Neck. & 187 & Pers. & 255 & Calodenilron Thunb. & tns $L d l$. \\
\hline & 200 & H. B.K. & 258 & Desv. & omm. \\
\hline$T h$. & 32.4 & & 78 & 190 & $K t h$ \\
\hline eck. & 232 & eyer. & 76 & Vent. & sia $D C$. \\
\hline & 71 & & 150 & Fisch. & 302 \\
\hline & 176 & & 78 & im $L$. & a Crantz. \\
\hline & 83 & & 129 & $D C$ & 299 \\
\hline am. et Schl. & 175 & & 153 & $R B r$ & ia $L$. \\
\hline C. & 187 & a Lag. & 200 & ium Desv. & ia $L k$. \\
\hline av. & 274 & & 36 & in Lag. & oea $B l$. \\
\hline & 215 & & 185 & s Labill. & $L$ \\
\hline & 221 & Less. & 187 & Presl. & A. Rich. \\
\hline & 315 & & 78 & na $R B$ r. & ria Lag. \\
\hline & 221 & 8 Cass. & 185 & Desv. & elia Rich \\
\hline & 52 & $R B r$ & 48 & nus Labill. & $\mathrm{Lk}$ \\
\hline$a m$. & 232 & $L$ & 187 & Desv. & a Nees. \\
\hline in Hall. & $2 \mathbf{I}$ & & 314 & $A g$ & na $L . \quad 103$ \\
\hline Bus: & 96 & & 78 & us Spach. & sia $\boldsymbol{R}$. et \\
\hline & 268 & Nees. & 187 & 160 & 232 \\
\hline Rick. & 286 & & 327 & ? 306 & 133 \\
\hline & $? 2$ & Cal & 109 & et. $-T h$. & psotrichum Elirb. 2 \\
\hline & 142 & nthemum $C$. & $\begin{array}{l}\text { A. } \\
325\end{array}$ & $\begin{array}{l}221 \\
325\end{array}$ & $\begin{array}{l}\text { ptosporium } L k \text {. } \\
\text { jtoum } L k \text {. }\end{array}$ \\
\hline & 127 & & 138 & 187 & Campuloa $P . B . \quad 42$ \\
\hline & 321 & & 187 & 233 & linium DC. 185 \\
\hline Juss. & 306 & Andr. & 251 & 183 & Desv. $\quad 42$ \\
\hline & 42 & $L$ & 106 & ium $A c h$. & era Sch. et \\
\hline & 185 & & 188 & 150 & 29 \\
\hline & 221 & Don. & 188 & ium $D C$. & as Roth. \\
\hline & 200 & achb. & 187 & $R B r$ & Pet.-Th. \\
\hline B. $K$. & 187 & et. & 311 & llum $D C$. & lia Sweet. \\
\hline & 234 & & 174 & is Lam." & \\
\hline & & Ldl. & ? 67 & ne $L k . \quad 221$ & \\
\hline & $\$ 15$ & & 69 & s R.ct Pav. 148 & lopus Brid. $\quad 21$ \\
\hline & 175 & isch. & 200 & Lag. $\quad 185$ & lostachys Kth. 141 \\
\hline & 78 & & 49 & iia Nutt. & Campylotheca Cass. 187 \\
\hline & $2 \geq 2$ & Callista Lour. & 88 & Calymperes Sw. & Campyncma Labill. \\
\hline
\end{tabular}




\begin{tabular}{|c|c|c|c|}
\hline & & & \\
\hline ium $R_{p h}$ & arlina $L$. & Castela Turp. & Cenia Comm. \\
\hline a Juss. & itzia Macuch. 184 & Castelia Cav. & Cenococenu Fr. \\
\hline marium $L$. & ica R. et Par. 39 & Castiglionia $R$. et Pav. 96 & Cenolophiun Koch. \\
\hline navalia $D C$. & armichaelia Grcv. $\quad 15$ & Castilleja Mut. & Cenolophon $B l$. \\
\hline indarum Rchb. & armichaëlia $R B r . \quad 221$ & Casuarina $L$. & Cenomyce $A c h$. \\
\hline Illea Labill. & armona Cav. ? 146 & Catabroga $P . B$. & Centaurea $L$ \\
\hline andollea Radd. & arolinea $L . f i l$. & Catachyon Ehrb. & urella Michx. \\
\hline inella P. Br. ? 302 & ia Endl. & Catagyna $R B r$. & uropsis $B o j$. \\
\hline ephora Juss. $\quad 187$ & Thunb. & Catalpa Juss. & Centotheca Desv. $\quad 49$ \\
\hline na $L$ & 118 Raf. & Catananche $L$. & Centrachaena Schott. 187 \\
\hline Annabis $L$ & H. $L 185$ & um $L k$. & ra $R B r$ \\
\hline mecoril Lam. & 43 & aertn. & $D C$ \\
\hline Juss. $\quad 115$ & Juss. & um Brid. & 8 Cass. 185 \\
\hline ellus Adans. 5 & rue Cass. 185 & & rum Cass. $18 \overline{0}$ \\
\hline ann. $\quad 175$ & 185 & 15 & in Don. 186 \\
\hline ss. $\quad 153$ & In Cass. 185 & 176 & Labill. \\
\hline l. $\quad 327$ & 85 & 210 & Don. \\
\hline t. Hil. Ufi & 18 Mich. & Ehrl. 21 & um Trin. \\
\hline$? 2$ & rev. & Irpus $P$ crs. 222 & a $R B r$ \\
\hline Floerk. 8 & 69) & Presl. & Tich. \\
\hline M. $\quad 221$ & 209 & & แщแ $S p r$. \\
\hline Figert. $\quad 316$ & 303 & 232 & L. \\
\hline iaert. 200 & 272 & & 177 \\
\hline 315 & 69 & & ra Rich. \\
\hline 129 & Grev. & & \\
\hline 178 & $x b . \quad 221$ & 200 & chrad. \\
\hline 314 & 314 & sk. ? 286 & $n n$ \\
\hline 148 & & & Inz. \\
\hline 115 & 184 & 32 & m Rehb. \\
\hline 221 & 184 & 2 & \\
\hline Piso. $\quad$ 5fi & 49 & & 18 \\
\hline bl. 299 & 200 & lichx. 323 & \\
\hline 201 & 220 & 225 & s. 4 \\
\hline 160 & 288 & & \\
\hline 306 & 42 & & \\
\hline$A u b l$. & 297 & 294 & Lk. \\
\hline L. $\quad 314$ & 232 & Pav. 295 & bill. ? 230 \\
\hline Im Salisb. 76 & 61 & 173 & lis. \\
\hline sv. $\quad 314$ & 325 & 209 & 8 Desv. \\
\hline 187 & $23 y$ & $32 t$ & \\
\hline 314 & 222 & n P'alis. & $\mathrm{Ag}$. \\
\hline hlchtd. & & & $B l$. \\
\hline 330 & $? 138$ & 307 & \\
\hline 289 & 222 & 249 & 261 \\
\hline & 200 & 116 & $22 f$ \\
\hline us $A l l$. & 210 & 210 & ra Horncm. 86 \\
\hline 184 & 185 & 187 & 207 \\
\hline 185 & 204 & Celosia $L$. & $L k$ \\
\hline & 176 & ? $148(129)$ & $w n .2$ \\
\hline 232 & In Lam. & & n $B l$. \\
\hline 166 & 116 & & irpus $L$. \\
\hline 196 & 187 & s Labill. & in Nees. \\
\hline 162 & astanea Gaertn. & nchrus $L$ & \\
\hline
\end{tabular}




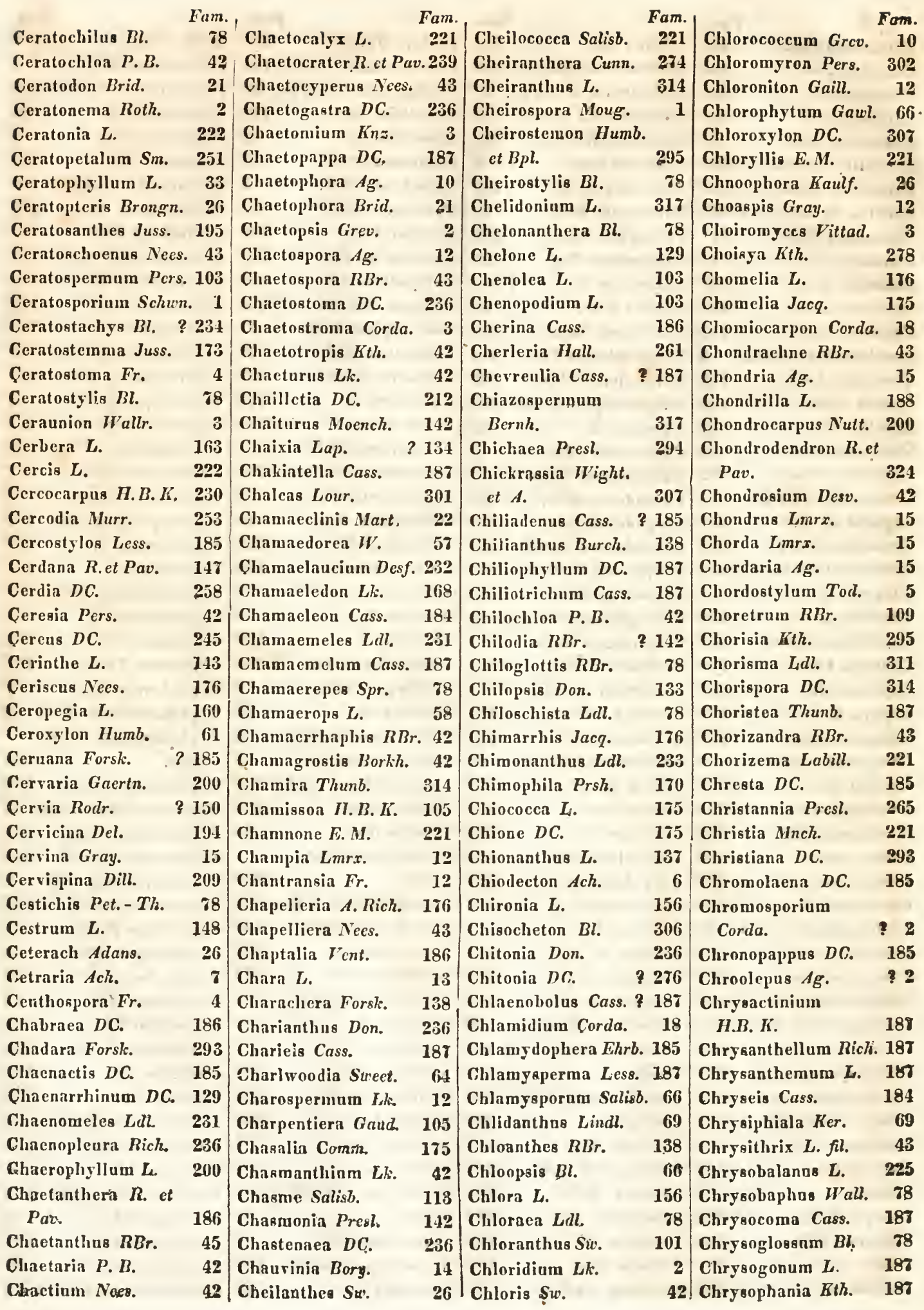




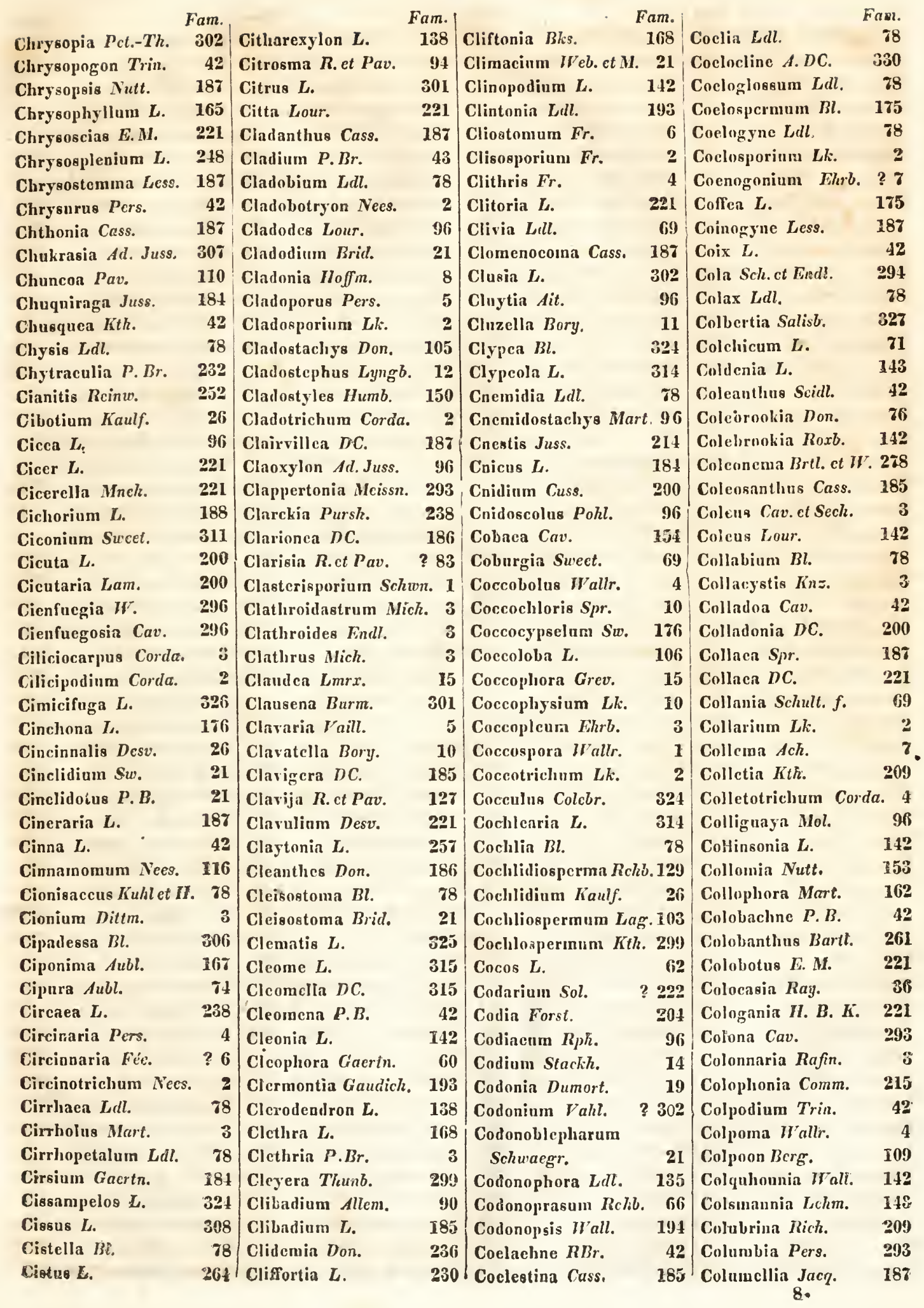




\begin{tabular}{|c|c|c|c|c|c|}
\hline Columellia $R$, et $P a v$. ? & $\begin{array}{l}\text { Fam. } \\
\text { ? 129 }\end{array}$ & Conoria H.B.K. & $\begin{array}{r}\text { Fam. } \\
266\end{array}$ & Cortusa $L$. & Coutoubea $A u b l$. \\
\hline Columnea $L . \quad$ ? & 135 & Conospermum Sm. & 113 & Gortusina $D C$. & Cowania Don. \\
\hline Colutea $L$. & 221 & Connstegia Don. & 236 & Corvisartia Mer. & Crafordia Raf. \\
\hline Colutia Mnch. & 221 & Conostounm Sw. & 21 & Coryanthes Hook. & Crambe $L$. \\
\hline Colynubea Salisb. & 80 & Conostylis $R B r$ & 72 & Córybas salisb. & Cranichis $S w$ \\
\hline Colythrnm Schott. & 278 & Conringria Host. & 211 & Corycarpus Zea. & Craniolaria $L . \quad 132$ \\
\hline Comardra Nutt. & 109 & Conulcum Rich. & 111 & Corycium Sw. & Craniosperinum Lehm. 143 \\
\hline Comaropsis Rich. & 229 & Convallaria $L$. & 64 & Corydalis $V$ cnt. & Craniotome Rchb. \\
\hline Comarim $L$ & 229) & Convoliulus $\mathrm{L}$. & 150 & Corylus $L$. & Crantzia Nutt. \\
\hline Combretum $L o f f l$. & 231 & Conyza $L$ & 187 & Corymbium $L$. & Crantzia Schreb. \\
\hline Comesperma Labill. & 272 & Cookia Sonner. & 301 & Corynandra Sehrad. & Crantzia $S w$. \\
\hline Comctes Burm. & 96 & Cooperia Herb. & 6!) & Coryne $F r$. & Craspedia Forst. \\
\hline Commelina $L$ & 49 & Copaifera $L$. & 222 & Corynclia Ack. & Craspedon Fée. \\
\hline Commersona Sonzer. & 232 & Copisma E.M. & 221 & Corynclla $D C$ & Crassocephalum \\
\hline Commersonia Forst. & 291 & Coprinus Pers. & 5 & Corynephora $\mathrm{Ag}$. & Minch. \\
\hline Commia Lour. & 98 & Coprosma Forst. & 175 & Coryuephorns $\boldsymbol{P} . \boldsymbol{B}$. & Crassula $L$ \\
\hline Commidendrum $D C$. & 187 & Coptis Salisb. & 325 & Coryneum Fr. & Crataegus $L$ \\
\hline Comocladia $P . B r$ & 219 & Corallopsis Grev. & 15 & Corynitis Spr. $\quad \mathbf{2 2 1}$ & Crataeva $L$ \\
\hline Comolia $D C$ & 236 & Corallorhiza Hall. & 78 & Corynodesmium Wallr.? 2 & Craterella Pers. \\
\hline Comostemum Necs. & 43 & Curchorus $L$. & 293 & ostylis Mart. $\quad \mathbf{2 6 6}$ & Crateria Pers. \\
\hline Comparettia $P \ddot{p p p}$. et $E$ & E. 78 & Cordaca Nces. & 19 & Corgpha $L$. & Craterium Trentep. \\
\hline Compsanthus Spr. & 81 & Cordia $L$ & 148 & Corysantlies $R B r$. & Crateromyces Corda. \\
\hline Compsoa Den. & 71 & Cordiera A. Rich. & 175 & Coscinium Colebr. & Cratoxylum $B l$. \\
\hline Comptonia Bks. & 83 & Cordiopsis Ham. & 147 & Cosciniun Findl. & Cremanium Don. \\
\hline Conami $A u b l$. & 96 & Corilylia Lour., Pers. ? & 2222 & Coscinodon Spr. & Cremastra $L d l . \quad 78$ \\
\hline Conanthera $R$. et Pav. & $? 66$ & Cordyline Comm. & 64 & Cosmea $W$ & Crenacephalum Cass. 185 \\
\hline ibun Rich. & 96 & Gordylocarpus Desf. & 314 & Cosmelia $R B r$. & Cremolobus $D C . \quad 314$ \\
\hline Conchiun sm. & 113 & Corcma Don. & 207 & Cosmia Doml. & Crenea $A u b \psi$ \\
\hline Conchnearpus Mik. & 28 & Coremium Lk. & 2 & Cosmibuena $R$.et & Crenias $S p r$. \\
\hline alia Cav. & 209 & s $L$ & 187 & Pav. & Creodus Lour. \\
\hline Condalia R. et $\boldsymbol{P} a v$. & 186 & gyne $D C$. & 187 & Casmos Cav. & Crepidium $\mathrm{Bl}$. \\
\hline Condaminea $D C$ & 176 & drum $L$. & 200 & Cosmus Pers. & Crepis $L$. \\
\hline Condylocarpus $I$ off $\mathrm{m}$. & .800 & Coriaria $L$. & 282 & Cossinia Comm., Spr. $\mathbf{2 8 9}$ & Grescentia $L$ \\
\hline Conferva $A g$. & 12 & Coriou $L k$. & 200 & Costaria Grev. & Gressa $L$. \\
\hline Conger Roxb. & 138 & Coris $L$ & 126 & Costus L. = & Cribraria Schrad. \\
\hline Coniangium $F r$. & 6 & Corispermum $L$. & 103 & Cotoneaster Med. & Crinitaria Less. \\
\hline Coniocarpon $D C$. & 6 & Cornicularia Ael. & 9 & Cottaca Kth. $\quad .42$ & Crinium Fr. \\
\hline Coniocybe $A c h$. & 8 & Cornucopiae $L$ & 42 & Cottendorîa Sehult.f. $\mathbf{5 6}$ & Crinodendron Mol, ? 29ะ \\
\hline Conioloma Floerk. & 6 & Coruulaca $D C$. & 103 & Cotula $L . \quad \quad ? \mathbf{1 8 5}$ & Crinonia $\mathrm{Bl} . \quad 78$ \\
\hline Coniophora $D C$. & 5 & Cornus $L$ & 202 & Cotyledon $L . \quad 255$ & Crinula Fr. \\
\hline Conioselinum Fisck. & 209 & Comutia $L$ & 138 & Cotylephora Meissn. ? 293 & Çrinum $L . \quad 69$ \\
\hline Coniosporiuna $L k$. & 8 & Coronilla $L$ & 221 & Conepia Aubl. $\quad 225$ & Criocephalus Selllechtd. 71 \\
\hline Coniothele $D C$ & 188 & Coronopus Hall. & 311 & Coulteria $\boldsymbol{H} . \boldsymbol{B} . \boldsymbol{L}$. & Cristaria Cav. $\quad 296$ \\
\hline Conium $L$. & 200 & loria Mart. & 12 & Couma Aubl. & Cristaria Sonn. \\
\hline Conjugata $L k$. & 12 & Correa $\$ \mathrm{Sm}$. & 258 & Coumarouna $\mathrm{Au} \mathrm{Ol}$. & Critanıus Less. \\
\hline Comnarus $L$. & 214 & Correia T'ell. & 281 & Coupoui $A u b l$ & Crithmum $L$ \\
\hline Conobea Aubl. & 129 & iola $L$. & 258 & Couratari Aubl. & Critonia Gaertin. \\
\hline Conocarpus $L$. & 110 & Corsinia Radd. & 16 & Couroupita Aubl. & Crocodia $L k$. \\
\hline alus Hill. & 18 & Cortesia Cav. & 150 & Coursetia $D C$. & Crocus $L$ \\
\hline Conoclinium $D C$. & 185 & Cortia $D C$ & 200 & Conrtoisia Nees. & Cronartium $F r$. \\
\hline Conohoria Aubl. & 266 & Cortica $F v$. & 5 & Coussapoa $A a b l$. & Crossandra Salisb. \\
\hline Conoplea Pers. & 1 & Corticium Pers. & 5 & Coussarea Aubl. & Crossolepis Less. $\quad 185$ \\
\hline Conopodium $D C$. & 200 & Cortinaria $\mathrm{Fr}$. & 5 & Contarea Aubl. & Crossopetalum P.Br. 210 \\
\hline
\end{tabular}


Fam.

Crossosteplium Less. 185

Crosentylis Forst. ? 232

Crotalaria $L$. 221

Croton $L$.

96

Crotonopsis Michr.

96

Crowea $S m$.

278

rrozophora Neck.

Crucianella $L$.

Crucigenia Morr.

Crucita $L o ̈ f l$.

Crudia $W$.

96

174

11

103

222

Cruikshankin Hook. ? 27t

Crumenaria Mart.

209

Cruminium Desv.

Crupina Pers.

221

184

175

Crusea A. Ricl.

Crybe Ldl.

Cryphaen Brid.

Cryphia $\mathrm{RBr}$.

Cryphiospermum P. $\boldsymbol{B}$.

Crypsis Ait.

Crypta Nutt.

Cryptandra $S m$.

88 Cunninghamia Rich.

21

142

Cunningliamia Schreb.

Cunonia $l$.

Cupania Juss.

$? 185$ Cuphen Jacq.

42 Cupia DC.

260 Cupressus $L$.

209 Curatella $L$.

Cryptarrhena $R B r . \quad 78$ Curculigo Gaerin.

Cryptina $\operatorname{Raf}$.

260 Curcuma $L$.

\begin{tabular}{lr|l} 
Cryptocarpha $R B r$. & 183 & Curtia Cham. \\
Cryptocarpus H,B.K. ? 104 & Curtiaia Ait.
\end{tabular}

Cryptocarya $\mathrm{RBr}$. 116 Curtogyne Haw.

Cryptochilus $\mathrm{Wall} . \quad 78$ Curtopogon P. B.

Cryptocaryne Fisch. $\quad 36$ Curupita Gm.

Cryptoglottis $B l$.

Cryptogramma $R B r$.

Cryptolepis $R B r$.

Cryptololus Spr.

Cryptomyces Grev.

Cryptopetalum Cass. 18

Cryptopodia Rochl.

Cryptopus Ldl.

Cryptosphaeria Grev.

Cryptosporiun $K n z$.

Cryptostegia $R B r$.

Cryptostenma $\mathrm{RBr}$.

Cryptostylis $\mathrm{RBr}$.

Cryptotaenia $D C$.

Cryptotheca $\boldsymbol{B l}$.

Cteisium Rich.

Ctenium Panz.

Ctenopteris $B l$.

Cuazonaria Corda.

Cubnea Schrcb.

Cucifera $D$ el.

Cucribalus $L$.

200

237

21

42

26

26

5

222 Cyathophorum Palis.

60 Cyathula Lour.

262 Cyathus Hall.
Fam.

5.

Cybbanthera Ham.

316 Cybelc Salisb.

291 Cybelinm Spr.

45 Cycas $L$.

195 Cyelamen $I$.

195 Cyclantlus Poit.

4 Cyclas Schreb.

168 Cycledium Wrallr.

78 Cyclubotlira Don.

36 Cycloderma $K l$.

185 Cyclomyces Knz.

187 Cyclophorus Desv.

200 Cyclopia Vent.

? 66

175

142

Cycloporan Presl.

Cyclostegia Benth.

Cyclotella fiütz.

80 Cyclospermum Lag.

Cycnoches Ldl.

251 Cydonia Tourn.

289 Cylactis Raf.

237 Cylichnium Wall.

176

80

327

68

86

156

210

255

42

232

151

278

187

201

165

165

6fi

69

321

320
185

185

49

66

185

26

Cylindrolobus $B l$.

Cylindropus Necs.

Cylipogon Raf.

Cylista Ait.

Cylizoma Neck.

Cymaria Benth.

Cymation spr.

Cymbachne Retz.

Cymbaria $L$.

Cymbella Kütz.

Cymbidium Sw.

Cymbonot us Cass.

Cymboplora Kütz.

Cymbopogon Spr.

Cyminosma Gacrtn.

Cymodocen Koen.

Cymopterus Raf.

Cynanchum $L$.

Cynara $L$.

43 Cynoctonum $\mathbf{G m}$.

172 Cynndon Brid.

16

Cynodon Rich.

Cynodontium Brid.

78 Cynoglossum $L$.

105

Cynometra $L$.

3 Cynontodium Hedw.
Fam.

129

113

Cynoptaallus Fr.

Fxж.

23

118

79 Cynosciadium DC. 200

126 Cynosurns $L$. 42

39 Cynthia Don. 188

222 Cypella $H_{0 o k} .74$

4 Cyperus Linn. 43

67 Cypliclium Ach.

3 Cyphella $F r$. 5

5 Cyphia Berg. $\quad 190$

2fi Cypripedium L. $\quad 78$

221 Cyrilla $\mathrm{Hl}$ cr. 129

78 Cyrtandra Forst. $\quad \mathbf{1 3 4}$

142 Cyrtanthus Ait. 69

11 Cyrtanthus Schrcb. 176

200 Cyrtochilum H. B.K. 78

78 Cyrtodon $R B r$. 21

231 Cyrtolepis Less. 185

22y Cyrtopera Ldl. 78

3 Cyrtopodium Brid. 21

Cylicodaphne Necs. 116 Cyrtopodium $R B r . \quad 78$

Cylindria Lour. ? 113

Cyliudrocline Cass. ? 187

78

43

Cylindrosporium Grcv. 1

221

Cymbanthes Salisb.

221

221

142

71

129

187

278

32

200

160

184

163

21

143

222

222

Cynomorium Mich. 118
71

42

11

88

11

42

21

42

Cyrtopus Brid. 21

Cyrtosia $\mathrm{Bl} . \quad \mathbf{7 8}$

Cyrtostylis $\mathrm{RBr}$. $\quad \mathbf{7 8}$

Cystantlue $\mathrm{RBr}$. $\quad \mathbf{1 7 2}$

Cysticapuos Bocrh. $\quad 316$

Cystop:teris Bernh. 26

Cistoseira $\mathrm{Ag}$. 15

Cytheris $L d l$. $\quad 78$

Cytinus $L$.

119

Cytisus $L$. 221

Czackia Bess. ? 66

Czernya Presl. 12

Dacrina Fr.

2

Dacryllum Bks. 81

Dacrymyees Nees. 5

Dactylacna Schrad. $\quad 315$

Dactylicapnos Urall, 316

Dactylis $L$. 42

Dactilium Nees. 2

Dantyloctenium $W$. 42

Dacdalea Pers. 5

Dahlia Cav. $\quad 187$

Dais $L$.

115

Dalbergia $L . \quad 291$

Dalea Gaertn. $\quad 140$

Dalea $L$. 201

Dalea $P . B r$. 183

21 Dalechampia $L . \quad 96$
Cytispora Ehrl.

Dahlia Thunb. 205 


\begin{tabular}{|c|c|c|c|}
\hline & & & \\
\hline 229 & Decumaria $L$. & tzia Kth. & ícksonia Herit. \\
\hline nplea Roxb. & eeringia $R B r$. & Desvauxia $R B r$. & Dicliptera Juss. \\
\hline altonia Hook. & efforgia $L a m$. & Detarium Juss. & \\
\hline amasonium Juss. & Deguelia $A u b l$. & Detridiun Nees. & Dicnemon Schwaegr. \\
\hline um Schreb. & Deidaınia Pet.-Th. & Deutzia Thunb. & Dicoccum Corda. \\
\hline 183 & Dejanira Cham. & Deverra $D C$ : & Dicoma Cass. \\
\hline 215 & elaria Desv. & Deyeuria Clar. & Diconangia Mitch. \\
\hline lirb. $\quad 80$ & Delesseria Lmr.x. & Diacalpe $B l$. & Dicoryphe Pet.-Th. \\
\hline anthus Gaertn.175 & Delima $L$ & Diacantha Less. & Dicraeia Pet. - Th. \\
\hline Br. $\quad 191$ & Delisea Lmr.r. & Diachea $F r$ & Dicranopteris Bernh. \\
\hline 27 & Deloderinm Cass. & Diadenium Pöpp. et $E$. & Dicrannpteris $B l$. \\
\hline 64 & a Don. & Diagramme $B l$. & in Hedw. \\
\hline 176 & m $L$. & Dialesta $I . B . K$. & Dic \\
\hline 42 & 187 & $? 222$ & us $L$ \\
\hline 238 & Fr. & pha Nutt. & Rafin. \\
\hline 115 & $H_{0} f f m$ & a Mart. & n Schrad. \\
\hline 26 & $D C$ & am. & Di \\
\hline 129 & 2 & 131 & $\mathrm{Ag}$ \\
\hline gtonia $D C$. & Desv. & 262 & a Grev. \\
\hline ? 148 & m Su. & 168 & os Hass. \\
\hline 115 & mus Nees. & 42 & a Dest. \\
\hline$\quad 12$ & im Bl. & Raf. & L Lmrx. \\
\hline era Presl.?.265 & m Bl. & 129 & Grev. \\
\hline$A g . \quad 12$ & on Benth. 317 & 74 & Dic \\
\hline 200 & osch.? 3 & 191 & llium Nees. \\
\hline & Wallr. 2 & Salisb. & 11 \\
\hline & 185 & $D C$ & Her. \\
\hline & Ilissn. & ? 232 & Pers, \\
\hline ? 48 & 36 & 8 Don. & Di \\
\hline 255 & s14 & Eckl. et Z. & 20 \\
\hline 89 & 176 & Ne€8. & Di \\
\hline & ? 142 & ? 299 & arad. \\
\hline 22 & $\mid r z . \quad 314$ & & lack. \\
\hline indl. & 26 & Bernhl. & Bt. \\
\hline 200 & 4 & 292 & a Desv. \\
\hline 26 & $h l . ? 175$ & ? 135 & \\
\hline ? $6 f$ & rpon Eschw. 7 & $D C$ & Did \\
\hline & 4 & 78 & m Desv. \\
\hline nd. & Nees. & Dic & n Necs. \\
\hline 36 & 221 & in Willem. & B. $K$ \\
\hline 15 & P.B. $\quad 42$ & lum Pet.- & \\
\hline 21 & R. et Pav. 148 & 212 & orkh. \\
\hline 291 & $W$ & 42 & \\
\hline 185 & Lmrx. & 221 & Di \\
\hline 78 & a Bory. & irev. & urn. \\
\hline & 21 & tis Fisch. & \\
\hline Wight. & $\mathrm{Ag}$ & 261 & \\
\hline & is $F h$ r & 150 & \\
\hline Forst. 232 & ta $D C$ & Ira Hik. & \\
\hline Br. $\quad 172$ & n Desv. & Nees & Dig \\
\hline & Mart. & 43 & \\
\hline Decodon $\mathrm{Gm}$. 237 & esmos Lour. & 129 & sus Cass. \\
\hline Decostea $I t$ ct Pav.? 220 & esmotrichum Bl. & eng Bich & \\
\hline
\end{tabular}


Diglyphis $B l$.

Digraphis Trin.

Dilatris $L$.

Dilepyrum Michx.

Dilivaria Juss.

Dillenia $L$.

Dillwynia $S_{m}$.

Dilochia Lall.

Dimereza Labill.

Dimeria $R B r$.

Dimetopia $D C$.

Dimia Spr.

Dimncarpus Lour.

Dimorplua $\mathrm{W}$.

Dimorpluandra Sclott. 223

Dimorplintheca Fail. 18z

Dinclua Del.

Dinema Ldl.

Dioclea H. B. K.

Dioclea $S p r$.

Diodia $L$.

Diomedea Cass.

Dionaea Ell.

Diorygma Fishw.

Dioscorea $L$.

Dinsma $L$.

Dionpyros $L$.

Diothonea $L d l$.

Diotig Desf.

Dintis Schreb.

Diotostepluus Cass.

Dipera Spr.

Diphaca Lour.

Diphyes $B t$.

Diphylleia Michr. ? 323

Diphysa Jacq.

Diplıyscium Web. et M. 21

Diplaclune $P . B$.

Diplacliyrium Nees.

Diplarrum $R B r$.

Diplanthera $R B r$.

Diplarrhena Eabilt.

Diplasia Rich.

Diplazium $S w$.

221

143

175

187

268

6

65

228

166

ช8

185

103

187

78

221

78

221

42

42

43

129

71

43

26

Diplectrum Pet. $-T h$. is

Diplocalymnra Spr. 150

Diplocentrum Ldl.

Diplochita $D C$.

Diplocoen Faf.

Diplocoma Don.

Diplocomiuı $V$ esै. et $\mathrm{N}$.

Dipladerma $E k$.

i87

21

21
Fam

Diplolaena $\mathbf{R B r}$.

Fam

Diplolepis $R B r$.

278 Distephanns Cass.

Fam.

I60 Distichia Brid.

Diplomeris Don.

78 Distomaea Spenn.

19 Distreptus Cass.

187 Distylis Gaudich.

289 Ditassa $K R r$.

Diplopappus Cass.

Diplopetalon Spr.

293 Ditaxis Vahl.

Diplophractum Desf.

Diplophyllum Lehm. 129 Dithrichum Cass.

42 Ditiola $\mathrm{Fr}$.

Diplopogon $R B r$
Diploprion Viv.

221 Ditmaria Spr.

Diplosastern Tausch. 187

Diplospora DC.

175

Diplosporium $L k$.

2 Dodartia $L$.

Diplostachyum $P$. B. 22 Dodecas $L$. fil.

Dipiostegium Don. 236 Dodecatheon $L$.

Diplostephinm Kth. 187 Dodonaca $L$.

Diplnstoma $L k$ :

Diplotaxis $D C$.

3 Doellingeria Nees.

314 Docmia $R B r$.

62 Doliclınema Neow.

187 Dolichos $L$.

237 Dolichostylis Cass.

78 Doliocarpus Rol.

42 Dolophragma Fenzl.

281 Dombeya Cav.

200 Dombeya Lamb.

182 Donacodes $B$.

26 Donatia Forst.

221 Donax $P . B$.

297

200

3

76

115

6

78 Doraena Thunb.

129

Disandra $L$.

Disarrhenun Labir.

Disceliun Brid.

Dischidia $\mathrm{RBr}$.

Dischimia Chois.

Disascapros Ehata. et $\operatorname{sech}$

Discopleura $D C$.

Discovia Raf.

Discmma Labilt.

Diserandra Bentl.

78 Disoxylum $\mathrm{Bl}$.

236 Disparage Gaerin.

42 Disperis $\mathrm{Su}$.

Disporum Salisb.

Dissodon Grev, et Arn.

3 Distephana Juss.
42 Doria Thunb.

21 Doritis Ldl.

160 Dorobaea Cass.

140 Doronicum $L$.

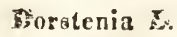

316 Doryantles Corr.

200 Doryenium Tourn.

314 Dothidea $F r$.

197 Douglassia Edl.

142 Dovera Eht.

306 Draba $L$.

185 Dracaena $E$.

78 Dracocephalum $L$.

? 71 Dracontium $L$.

21

187

195
Dracophyllum Labill. 172

Dracopis Cass.

Dracunculio Eess.

187
185

21

78

185

190

160

96

187

5

291

78

285

129

237

126

289

187

160

222

221

? 181

$32 \%$

261

294

80

76

248

42

200

175

200

187

26

221

? 148

210

187

78

387

187

91

69

221

4

126

II6

314

64

142

36

T85.

Fam.

Dracunculus Tourn. $\quad 36$

Draparnaldia Bory. $\quad 12$

Drapetes Lam. $\quad 115$

Drapiezia $B l$.

64

Drepania Juss. $\quad 188$

Drepanocarpus Meyer. 221

Drepanopliyllum

Koch.

200

Drepanophyllum Rich. 21

Drimia Jacq.

Drimgs Forst.

66

Drosera $L$.

329

Drozia Cass.

268

Drummendia $D C$

Drammondia $D C$

218

Drusa $D C$.

200

Dryandra $R B r . \quad \mathbf{1 1 3}$

Dryandra Thunb. 96

Dryas E.

229

Drymaria $W$.

261

Drymoplisila $R B r$.

64

Dryuaria Bory.

26

Dryobalanops Gaertn. 297

Dryopcia pct. - Th. $\quad \mathbf{7 8}$

Dryophilum Schwn. 3

Drypetes Vahl.

96

Drypis $L$.

ฉ62

Dryptodon Brid.

21

Dubautia Gaudich.

185

Duboisia $R B r$.

129

Duchesnea $S m$.

229

Dufourea $A c h$.

Dufourea H. B. $K . \quad 150$

Dufresnia $D C$.

180

Dugaldea Cass.

187

Duguetia St. Hit.

330

Dulraldea $D C$.

185

Duhamelia Pers.

176

Duliclsium Rich.

43 


\begin{tabular}{|c|c|c|c|c|c|}
\hline & & & Fam. & & \\
\hline & 217 & Ehretia $L$. & 146 & Embelia Burm. & Entostlyymenium Brid. 21 \\
\hline Dysoda Lour. & 175 & Ehrharta Sm. & 42 & Emblica Gaertn. & Enula Duby. \\
\hline Dysodia $D C$. & 187 & Ekcbergia Sparm. & 206 & Embolium Endl. & Enymonospermum \\
\hline Dysodiun Pers. & 187 & Elachistea Duby. & 12 & Embolus natsch. & Spr. \\
\hline Dysphania $R B r$. & 103 & laeagnus $L$ & 111 & Einbolus Hall. & Enzygostoma Gray. \\
\hline Dyssodia Lag. & 187 & Iaeis Jacq. & 62 & Embothrium Forst. 113 & Epacris Forst. \\
\hline & & Elaeocarpus $L$ & 292 & Eubryopteris Gaertn. 166 & Epaltes Cass. \\
\hline Earina Ldll. & 78 & Elncococea Comm. & 96 & Emericia R. ct Schult. 161 & Eperua $A u b l$. \\
\hline$R a f$ & 42 & Elacodendron Jacq. & 210 & Emex Neck. $\quad \cdot \quad 106$ & Ephebe Fr. \\
\hline xylon Lour. & 166 & num Kock. & $\mathbf{2 0 0}$ & Emilia Cass. & Ephedra $L$. \\
\hline Ebenus Comm. & 166 & Elaphoglossum Schott. & t. 26 & Eminium $B l$. & Ephiclis Schreb. \\
\hline Ebenus $L$. & 221 & Elaphomyces Necs. & 3 & Empedoclea St. Hil. & Ephippium Bl. \\
\hline phyllım P.Br. & 221 & hrium Jacq. & 215 & 207 & Epiandria Presl. \\
\hline mich. & $195^{\circ}$ & Elat & 62 & cum Sol. & Epibaterium Forst. \\
\hline carpus & & m $L$. & 195 & 78 & Epiblema $R B r$ \\
\hline & 133 & Ela & 260 & ria $\left.R c l_{2}\right\}$, & rus $\mathrm{Bl}$. \\
\hline a Ort. & 66 & ernma Forst. & 91 & 188 & $\mathrm{Bl}$. \\
\hline Cass. & 184 & Elc & 78 & a Gaerin. 68 & am Tod. \\
\hline$D C$ & 255 & El & 187 & 18 Labill. 314 & $\mathrm{CQ} B \mathrm{Bl}$. \\
\hline Much. & 187 & Panz. & 42 & 21 & \\
\hline ium Trin. & 42 & & 45 & 187 & 168 \\
\hline Desf. & 42 & $D C$ & 221 & m $L k$. & $L$ \\
\hline Ach. & 11 & Nees. & 43 & m Mart. & n $L$ \\
\hline ryon Corda. & 2 & $L k$ & 43 & 103 & \\
\hline us Salm. & 245 & pus $L$. & 185 & & 130 \\
\hline $18 B l$. & 265 & 8 Less. & 185 & 15 & \\
\hline a P. B. & 42 & a Willd. & 41 & a Lour. & \\
\hline lorus Rich. & 51 & hecd. & 76 & 78 & Gm. \\
\hline tena $H . B . K$. & 42 & . & 42 & iits. & a IJ.B $\mathrm{H}$ \\
\hline m Desv. & 221 & P. B. & 21 & Iuı $R l$. & \\
\hline an Desv. & 43 & $H$ & 185 & 116 & 175 \\
\hline$L$ & 200 & & 303 & & $L k$. \\
\hline gon $P: B$. & 42 & Eth. & 42 & $L k$. & \\
\hline$L$ & 184 & & 12 & 185 & \\
\hline mum $S w$ & 143 & & 306 & Levell. & $B$ \\
\hline era Sieb. & 36 & & 168 & 150 & $L$ \\
\hline our. & 96 & & 149 & 325 & 32 \\
\hline Desf. & 143 & rpus Käaulf. & 26 & 3 & 18 \\
\hline $1 \mathrm{~mm} B l$. & 78 & & 53 & 55 & 10 \\
\hline & 161 & & 303 & 187 & ch. \\
\hline$L$ & 143 & & 187 & 42 & 18 \\
\hline Hornm. & 15 & & 4 & 42 & 18 \\
\hline & 187 & & 142 & 289 & Less. \\
\hline Gaertn. & 187 & & 281 & 160 & 21 \\
\hline $\mathrm{Ag}$. & 12 & & 187 & 223 & la $R_{B}$. \\
\hline Ina Vaucís. & 14 & & 42 & 80 & us $M . B$. \\
\hline$F r$ & 4 & J & 43 & 293 & \\
\hline & 42 & B P. B. & 43 & Ehrb. & e $R_{B} r$ \\
\hline a Cass. & 185 & ${ }_{18} \mathrm{~W}$ & 42 & ha Fée. & thus Michr. \\
\hline Salisb. & 221 & Michx. & 131 & pla $L k$. & Erica $L$ \\
\hline & 26 & Desv. & 42 & n $B l k s$. & Erigenia Nut?. \\
\hline & 187 & ppus Cass. & 185 & lium Wallr. 1 & Erigeron $L$. \\
\hline hrenivergia Mart. & 276 & Iytrophorns P. B. & 21 & Entosthodon Schwaegr. 21 & Erinacea Lmrs. \\
\hline
\end{tabular}




\begin{tabular}{|c|c|c|c|}
\hline & & & \\
\hline eum $L k$ & irythrina $L . \quad 221$ & Eunnuia $D C$. & Exidia Fr. \\
\hline rinus $L$ & irythrochiton Nees. ct & Euosma $A n d r$. & Exilaria Grev, Kutz. 11 \\
\hline riobotrya Ldl. & Mart. $\quad 278$ & Euosmia II. B. K. & Exitelin $B l . \quad$ ? 29 \\
\hline calia $S m$. & rythrodanuu & Euosmus Nutt. & Exoacintha Labill. \\
\hline 47 & Pet. - Th. & Euopir Cass. & Exocarpus Labill. \\
\hline halus $L$. & irythrodea $B l$. & Suparca Bks. & Exormatostoma Gray. \\
\hline Ius $R B r$. & Erythronium $L$. & Supatorium $L$. & Exosporium $L k$. \\
\hline tena $A r n$. & Erythropalum $\mathrm{Bl} . \quad 195$ & Euphorbia $L$. & Exostemina $D C$ \\
\hline H. B. $K$. & eum $B B r .223$ & iuphoria Comm. & Eysclia Rchb. \\
\hline sis $P . B$ & psis Ldll. $\quad 294$ & $\sin L$ & lardtia II. B.K. 22 \\
\hline 287 & rhiza Michx. & gyne $D C$. & Cystathes Lour. $\quad 28$ \\
\hline 42 & aun & Salisb. & Taba $D C$ \\
\hline 295 & 265 & atia $R B r$. & Fabiana $R$ et $P$ vi. \\
\hline & hlchtd.71 & l. & Fabricin Graertn. \\
\hline 289 & 283 & Aurya Thunb. & Tabricia Scop. \\
\hline 106 & 219 & ale Satisb. & icia Thunb. \\
\hline 294 & ziil Cham. & Ira Forst. & nin Radd. \\
\hline 295 & 232 & lie Cham. & $? 18$ \\
\hline . 43 & 129 & $?: 311$ & 22 \\
\hline Jag. 187 & & sis Brid. & ia $L$ \\
\hline nth. $\quad 142$ & a kth. $\quad 278$ & 187 & yrum Gaertn. \\
\hline 21 & B Bl. ? 293 & psis DC. & \\
\hline 221 & 185 & & \\
\hline Jacq. $\quad 66$ & 187 & $? 279$ & ia Riv. \\
\hline 185 & $? 293$ & 188 & $G m$. \\
\hline 181 & 133 & Im Salisb. 113 & Ila Brot. \\
\hline 278 & 76 & & ria Bess. \\
\hline$t$ Findl. 295 & 185 & & L. fil. \\
\hline 185 & & & ea A. Rich. \\
\hline 200 & 202 & 69 & \\
\hline 135 & 232 & 21 & 31 \\
\hline 291 & 316 & & ta Giray. \\
\hline 175 & Mart. 28y & 221 & \\
\hline 236 & is Btl. et Wdl. 278 & & 23 \\
\hline 175 & r. 221 & 190 & uss. \\
\hline 113 & 185 & 187 & 18 \\
\hline 311 & 185 & 292 & 18 \\
\hline 314 & 129 & & \& $F r$ \\
\hline 299 & $R B r$ & 314 & In Gacrin. \\
\hline 266 & & 42 & 18 \\
\hline 21 & 66 & 185 & \\
\hline 314 & & 814 & \\
\hline 314 & 69 & 185 & Radd. \\
\hline & Cav. & 178 & \\
\hline & ua $R a f$. & Pöpp. ct E. & Lag. \\
\hline lia $L k$. & udema Inumb. & & a R. et Pav. \\
\hline 221 & 232 & 278 & 17 \\
\hline 150 & 232 & 150 & $\mathbf{3 0}$ \\
\hline 200 & & 210 & $L$ \\
\hline 3 & 78 & 156 & la Roxb. \\
\hline 314 & 200 & 143 & 200 \\
\hline & & & \\
\hline 56 & & & \\
\hline
\end{tabular}




\begin{tabular}{|c|c|c|c|}
\hline Fam. & & Fam. & \\
\hline Feuiliea $L$ & Fothergilla $L$. fil. & Gabertia Gaudich. & Gastonia Comm. \\
\hline Fibigia Koel. & Fougerouxia $D C$. & Gadelupa $L a m$. & Gastridium Lyngb. \\
\hline IFibigia Mcd. & Fouquiera II. B. K. & Gaertnera Lam. & Gastridiun $P . B$. \\
\hline Fibraurea Lour. & Fourcroya Vent. & Gaertnera Schreb. & Gastrocarpha Don. \\
\hline Fibrillaria Pers. & Foreolaria $D C$ & Gagea Ker. & Gastrochilus Wall. \\
\hline Ficaria Dill. & Fragaria $L$ & Gagnelina Neck. & Gastrodia RI3r. \\
\hline Ficinia Schrad. & Fragillaria $L y n g b$. & Galınia Forst. & Gastroglottis $B l$. \\
\hline Ficus $L$ & Fragoкa $R$. ct Pav. & Gaillardia Foug. ' 187 & Gastrolobinn $R L r$. \\
\hline Fieldia Gaudich. & Franciscea Pohl. & Gaillardotella Bory: 10 & Gastronema Sims. \\
\hline Filago $L$ & Francoa Cav. & Gaillonella Bory. $\quad 11$ & Gástrophycus $L k$. \\
\hline Fimbriaria Nees. & Francoeuria Cass. & Gaillonia A. Rich. 175 & Gatyona Cass. \\
\hline riaria St. Hil. & Frangula Tourn. & Gaimardia Gaudicl. $\quad 44$ & Gándichaudia $K^{\prime} t h$. \\
\hline istylis Rich. & $\operatorname{nia} L$ & Gaissenia Raf. & Gaudinia $P . B$ : \\
\hline huthia Nees. & Franklandia $\boldsymbol{R B r}$. & Galactia $P . B r$ & Gaultheria $L$. \\
\hline Firmiana Marsgl. & Franklinia Marsh. $\quad 299$ & Galactites Moench. 184 & Gacira $L$ \\
\hline Fischera $D C$. & Franseria Cav. ? 90 (185) & Galactodendron H.B.K. 91 & Gauticra Vittad. \\
\hline Fischera Lag. & ra Michs. $\quad 156$ & Galanthus $L$. & Gavilea $P_{\ddot{p} p p .}$ \\
\hline Fischiera $S w$ & Fraxinus $L$ & Galardia 'Lam. & Gaya Gaud. \\
\hline Fissidens Hedw. & Frenela Mirb. & Galasia Cass. & Gay: $K t h$. \\
\hline Vissilia Comm. & Fresenia $D C . \quad ? 185$ & Galatea Less. & Gaya Spr. \\
\hline Fissurina Fće. & Freycinetia Gaudich. 40 & Galatella Cass. & Gaylussacia $\boldsymbol{H} . \boldsymbol{B}: \boldsymbol{K}$. \\
\hline Fistulina Bull. & Freziera $S w . \quad 299$ & Galax $L$ & Gazania Gaertn. \\
\hline Flabcllaria $\mathrm{Cav}$. & Fridericia Mart. & Galaxia Thunb. & Geanthus Reinu. \\
\hline Flabellaria Chev. & Friellandia Cham. & Galeandra $L d l$. & Geaster Mich. \\
\hline Flabellaria Lmrx. & Friesia $D C$ & Galega $L$ & Geastrum D Desv: \\
\hline Flacourtia Her. & Friesia Spr. & Galcobdolon Huds. & Gecria $B l$. \\
\hline laria $L$. & Fritillaria $L$. & Galeola Lour. & Geigeria Griessl. \\
\hline Flaveria Juss. & Froelichia Juss. & sis $L$ & $\operatorname{loma} R B r$ \\
\hline Flemingia $R_{0} x b$. & Bert. ? & erdon $W_{e} b$ & Geissorhiza Ker. \\
\hline Flindersia $R B r$. & Frustulia $\mathrm{Ag}$. & Galera $B l$. & Gcitonoplesium Cunn. \\
\hline Floccaria Grev. & Fucastrum $D C$. & Galinsogaea $\boldsymbol{R}$. et $\mathbf{P a v}$, & Gelidium Lmrx. \\
\hline Floerkea Spr. & Fuchsia $L$ & Zuce. $\quad 18 \gamma^{\prime}$ & Gelenium Gaertn. \\
\hline Florestina Cass. & Fucus $L ., A g$. & Galipea $A u b l$. & Gelonium Roxb: \\
\hline Flosoopa Lour: & ia Juss. & Galium $L$ & Gelseminum Juss. \\
\hline Flotovia $S p r$. & Fuirena Rottb. & Galopina Thunb. & Gemella Lour. \\
\hline Flourensia $D C$. & Fulcaldea $P_{\text {oir. }}$ & Galorheus $F r$. & Gemmularia Rafin. \\
\hline Flüggea Rich. & Fuligo Hall. & Galphiınia Cav. & Genea Vittad. \\
\hline Flüggea $\|$ & Fullartonia $D C$. & Galurus Spr. & Genesiphylla tier. \\
\hline Plivialis Pers. & Fumago $P$ ers. & Galvania Vand. & Genetyllis $D C$ \\
\hline Focniculun $A d . \quad 200$ & Fumaria $L$. & Galvezia R. ct Pav. & Geniosporum Wall. \\
\hline Foetidaria St. Hil. 3 & Funaria $l l c d w$. & Gamocarpha $D C$. & Geniostona Forst. \\
\hline Foetidia Comm? ? 232 & Funkia Spr. & Gamolepis Less. & Genipa $L$ \\
\hline Fontanesia Labill. 137 & Furcaria $D c s v$. & Ganitrus Gacrtr. & Genista $L$ \\
\hline Fontinalis $L$ & Furcellaria $L m r x$. & Garcia $V a h l$. & Genlisia Rchb. \\
\hline Forbesia Fickl. & Fusanus $L$ & Garciana Lour. & Genoplesiun $\boldsymbol{R}_{B r}$. \\
\hline Forgesia Comm. & Fusarium Nces. & Garcinia L, A. Rich. 302 & Gentiana $L$ \\
\hline Fornicium Cass. & Fuscina Schrk. & Gardenia Fll. & Gentianella Borkh. \\
\hline Forrestia A. Ricki & Fusicoccum Corda. & Gardneria Wall. & Genuflexa $L k$. \\
\hline Forrestia Raf. & Fusiconia $\boldsymbol{P} . \boldsymbol{B}$ & Gardoquia R. et Pav. & Geijera Schott. \\
\hline Forskohlea $L$ & Fusidium Fr. & Garidella $L$. & Geochorda Cham. \\
\hline Forstera $L$. fil. $\quad 189$ & Fusidium $L k$. & Garuga Rorb: & Geodorum Jacks. \\
\hline Forsythia Vahl. 137 & Fusisporium Fr. & Garuleun Cass. & Geoffroya Jacq. \\
\hline Fotlıcrgilla $A u b l$. & & Gasteria Haw. & Geoglossam Pers. \\
\hline
\end{tabular}




\begin{tabular}{|c|c|c|c|c|c|}
\hline Geonoma ${ }^{\prime}$ & $\begin{array}{r}\text { Fam. } \\
61\end{array}$ & Globulen Haw. & Gomphus $F r$. & Grielum $L$ & $\begin{array}{r}\text { Fam. } \\
228\end{array}$ \\
\hline Geoplita Don. & 178 & Glochidion Forst. & Gomutus $k p h$. & Griffinia Ker. & 69 \\
\hline Geopyxis Pers. & 5 & Gloionema $A g$ & Gonatocarpus $W$ & Griffithia $R B r$. & 21 \\
\hline Georchis Ldl. & 78 & Gloiodictyon $\mathrm{Ag}$. & Gongora $R$ et $P a v$. & Griffithsia $A g$ & 12 \\
\hline Georgia $s_{p} r$ & 187 & Glomera $B l$. & Goniocarpus T/kunb. & Grimaldia Schrk. & 222 \\
\hline Georgina $W$ & 187 & Glonium Mühlb. & Goniocheton $\mathrm{Bl} . \quad 306$ & Grimmaldia Radd. & 18 \\
\hline Geracium Rchb. & 188 & Gloriosa $L$ & Goniophlebiun Schott. 26 & Grimmia Ehrh. & 21 \\
\hline Geranium $H \mathrm{cr}$. & 311 & Glossarrhen Mart. & Goniosporium $L k . \quad 2$ & Grindelia $\boldsymbol{W}$ & 187 \\
\hline Gerardia $L$ & 129 & Glossaspis Ldl. & Goniosporum $L k$. & Griesclinia Neck. & 221 \\
\hline Gerascanthus Mart. & 147 & Glossocardia Cass. & Gongycladon $L k$. & Grislea Löffl. & 237 \\
\hline Gerbera Gron. & 187 & Glossocomia Don. & Gonocarpus Ham. & Grobya Ldl. & 78 \\
\hline Gerbera Lour. & 191 & Glossodia $R B r$. & Gonogona $L k$ & Grona Lour. & 291 \\
\hline Germanca Lam. & 142 & Glossogyne Cass. & Gonolobus Rich. & Gronovia $L . \quad$ ? & 243 \\
\hline Gerontogea Cham. & & Glassoma Schreb. $\quad 202$ & Gonospermum Less. & Grossularia $A$. Rich. & 214 \\
\hline et Schl. & 186 & Glossopetalum Schreb. 209 & Gonotheca $B l$. & Groutia Guill. et Per. & 300 \\
\hline Geropogon $L$. & 188 & Glossospermum Wall. 291 & Gonus Lour. & Grubbia Berg. & 109 \\
\hline Geruma Forsk. & ? 308 & Glossostemon Desf. $\quad 294$ & Gonyanthes $B l$. & Grumaria Knz. & $? 2$ \\
\hline Gessneria $L$. & 135 & Glossostylis Cham. & Gonytrichum Nees. & Grumilea Gaertn. & 175 \\
\hline Gethyllis $L$. & 69 & Glottidium Desv. & Gonzalea Pers. & Guadua $K t h$. & 42 \\
\hline Gethyrium Salisb. & 76 & Gloxinia $H \epsilon r$. & Goodenia $S m$. & Guajacum Plum. & 276 \\
\hline Getonia Koxb. & 110 & Gluta $L$ & Groodia Salisb. & Guajava Tourn. & 232 \\
\hline Geum $L$. & 229 & Glyceria $R B r$. & Goodyera $R B r$. & Guapurium Juss. & 232 \\
\hline Glinia $S w$ & 138 & Glycine $L$ & Gordonia Ell. & Guardiola $\boldsymbol{H} . B$. & 187 \\
\hline Gibbera $F r$. & 4 & Glycosmis Corr. & Gorteria $L$ & Guarea $L$ & 300 \\
\hline Gifula Cass. & 185 & Glycyrrhiza $L$. & Gossampinus Sch. & Guatteria $R$. et Pav. & 330 \\
\hline Gigalobinun $P . B r$. & 223 & Glyphis $A c h$ & ct Endl. & Guazuma Plum. & 294 \\
\hline Gilia R. et Pav. & 153 & Glyphocarpus $R B r$. & Gossypium $L$. & Guepinia $F r$ & 5 \\
\hline Gilibertia $\mathbf{G m}$ & 306 & Glyphomitrium & ofreda Vent. & da Vent. & 175 \\
\hline Gilibertia R. et Pav. & 201 & Schwacgr. & $\operatorname{ania} L$ & Mol. & 113 \\
\hline Gillenia Moench. & $22 \pi$ & Gmelina. $L$ & Gouffeia Robill. & Guichenotia Gay. & 294 \\
\hline Gilliesia Linull. & $? 66$ & Gnaplsaliuın $L$. & Goupia $A u b l$. & Guidonia Plum. & 306 \\
\hline Gimbernatia R. et Pav. & v. 110 & Guephosis Cass. & Govenia Ldl. & Guiera Juss. & 234 \\
\hline Ginannia Scop. & 222 & Gnetum $L$ & Graenia Hook. & Guilandina $L$ & 222 \\
\hline Ginkgo Thunb. & 81 & Gnidia $L$ & Graffenricda $D C$. & Güldenstaedtia Fisch. & .221 \\
\hline Ginoria Jacq. & 237 & Gochnatia Kth. & Grammanthes $D C$. 255 & Guilielma Mart. & \\
\hline Girardia Gray. & 12 & Godinella Lestib. & Grammarthron Cass. 187 & Guilleminea $\boldsymbol{H} . \boldsymbol{B} . \boldsymbol{K}$. & \\
\hline Girodella Gaill. & 11 & Godovia Pers. ? 29y & Grammatophyllum $\mathrm{Bl} .78$ & Guindilia Gill. $\quad$ ? & 278 \\
\hline Gisekia $L$ & 104 & Godoya R. et Pav. ? 299 & Grammica Lour. $\quad 151$ & Güntlıeria Trev. & 16 \\
\hline Gisopteris Bernh. & 24 & Goeppertia Nees. $\quad 70$ & Grammitis $S w$ & Guioa $C a v$ & 289 \\
\hline Githago Desf. & $2 \delta 2$ & Goldbachia $D C$. & Grammonema $\mathrm{Ag} . \quad 11$ & Guizotia Cass. & 187 \\
\hline Glabraria $L$. & 116 & Golulbachia Trin. & Grammosciadium DC. 200 & Gundelia Tourn. & ? 181 \\
\hline Gladiolus $L$. & 74 & Gomara $R$. et $P a v$. & Grangeria Comm. $\mathbf{2 2 5}$ & Gundelsheimera & \\
\hline Glaphyria Jack. & 232 & Gomeza $R B r$ & Graphephorum Desv. 42 & Cass. & $218 \pm$ \\
\hline Glaucium $L$. & 317 & Gomortega $\boldsymbol{R}$. et & Graphiola Poit. 4 & Gunnera $\boldsymbol{L}$. & $? 91$ \\
\hline Glechoma $L$. & 142 & Pav. & Graphis Ach. & Gunnia Ldl. & 78 \\
\hline Glechon Spr. & 142 & Gomozia Mut. & Grateloupia $\mathrm{Ag}$. & Gussonea $A$. Rich. & 78 \\
\hline Gleditschia $L$. & 222 & Gomphia Schreb. & Gratiola $L$ & Gussonia Presl. & 43 \\
\hline Gleichenia $S m$. & 25 & Gomphocarpus $R B r . \quad 160$ & Graumüllera $R c h b$. & Gustavia $L$ & 232 \\
\hline Glinus $L$. & 256 & Gompholobium $S m . \quad 221$ & Greggia Gaertn. & Gutierrezia Lag. & 187 \\
\hline Gliotrichum Eschw. & $\mathbf{2}$ & Gomphonema $A g . \quad 11$ & Gregoria Duby. & Guzmannia R. et Pav. & 50 \\
\hline Glissanthe Salisb. & 76 & Goinphostemma Wall. 142 & Grevillea $R B r$. & Gyalecta $A c h$. & 6 \\
\hline Globba $L$ & 86 & Gomplostylis Wall. 78 & Grewia $L$. & Gymnadenia $R B r$. & 78 \\
\hline Globularia $L$. & 123 & Gomphrena $L$ & Grias $B$ & Gymnandra Pall. & 129 \\
\hline
\end{tabular}


Gymnanthemum Cass.185

Gymnanthera $R B r . \quad 160$

Gymnanthes $S w$.

Gymnema $R B r$.

Gymnocarpum Forsk. 258

Gymnocephalus

Schwaegr.

Gymnocladus Lam.

Gymnocline Cass.

21

222

187

Gymnocoronis DC. 185

Gymnodiscus Less, 187

Gymnogranume Desv. 26

Gymnogynum P. B. $\quad 22$

Gymnolomia Less. 18 ร

Gymnomitrium Nees. 19

Gymnopera Don, 218

Gyunopogon P. B. 42

Gymnopsis $D C$.

Gymnopteris Bernh.

Gymuosiphon Bl.

Gymnosperma Less. 187

Gymnosphaera $B l$.

Grymosporangium

Hedw.

Gymnostachys $R B r . \quad 36$

Gymnostephium Less. 187

Gymnosticbum Schreb. 42

Gymnostòmuı IIcdw. 21

Gymnostyles Juss. ? 185

Gymnothrix $P$. $B$.

Gymnotroclies $B t$.

Gynandropsis $D C$.

302

Gynantlist: ophe Poit. 222

Gynema Raf.

? $18 \%$

Gynerium H. B. K. 42

Gynestum Poit.

Gyneteria Spr.

Gynocardia Roxb.

Gynochtodes $B l$.

Gynoon Ad. Juss.

Gynopachys $B l$.

Gynoplenra Cav.

Gynopogon Forst.

Gynostemma $\mathrm{Fl}$.

Gynoxy' Cass.

Gynura Cass.

Gypscopliila $L$.

Gyrinops Gaertn.

Gyrocarpus Jacq.

Gyrocephalus Pers.

Gyrodon Opat.

Gyrolophium Kns.

Gynomium $W^{*}$ allr.

? 187

? 315

175

96

186

198

163

324

187

185

112

234
Fam.

96

160

Fam.

Gyrophora Ach.

Gyropodium Hitchc

Gyrostemon Desf. ? 293

Gyrostomum Fr.

Gyrotriclım Spr.

Habenaria $W$.

Lablizia $M . B$.

Habranthus Herb.

IJabzelia $A$. $D C$.

IIacquetia Neck.

IIacmadictyon $L d l$.

Haenanthus $L$.

II aemaria $\boldsymbol{L d l}$.

Hacmatococeus $\mathrm{Ag}$.

Haematoxylon $L$.

Haemocharis Salisb. 299

72

Haenkea R.et Pav. ? 203

Haenkea Salisb.

257

Hagenbachia Nees. ? 72

IIagenia Eschw.

Hakea Schrad.

Halenia Borkh.

Halesia $L$.

Halianthus $F r$.

IIalidrys Lyngb.

IIalimodendron Fisch. 221

Halleria $L$.

Hallia Thunb.

Halocnemon $\boldsymbol{M}$. $B$.

Hlalogaminm Nees.

IIalophila Pet.-Th.

Haloragis Forst.

262

5 Hapalanthus Jacq.

5 Haplache Presl.

5 Laplaria $L k$.

- II aplocarpha Less.

78

103

69

330

200

69

ร8

10

113

156

167

261

15

103

129

221

103

42

32

253

IIalterophora Endl.

Ualymenia $\mathrm{Ag}$.

Halyseris Targ.

Llaunadryas Comm.

IIamamelis $L$.

IIambergera Scop.

Hamelia Jaeq.

Hamelinia A. Rich ?

Hamiltonia Rosb. 175

Hamiltonia $\boldsymbol{W}$.

Itamulium Cass.

Hancornia Gom.

Hanguana $B l$.
109

187

162

? 46

49

42

187
Haemocarpus Noronh. 303

Haemodorum Sm.

Halimus Wallr.

7

Haplomitrium Nees. 19

Haplostellis A. Rich. 78

6 Haplost ephium Don. 188

2 Haplostephiun Mart. 185

22

7.
Haplostylis Nees.

Harrisonia llook.

Harrisonia $R B r$.

Hartighsea Ad.Juss. 206

Hartmannia $D C$.

Hartogia Thunb.

Hasselquistia $L$.

43

2

222

303

303

187

188

221

42

289

131

160

280

187

210

200

Hasseltia

II. B. $K$.

IIastingia $S m$.

IIavetia Kth.

Haworthia Salm.

Hlaxtonia Hort.

Hayia Moc. ct Sess. ? 238

Haylockia Herb.

Haynea $W$.

Hebanthe Mart.

Hebe Juss.

Hebcandra Bonpl.

Hebeclinium $D C$.

Hebelia $\boldsymbol{G m}$.

IIebenstreitia $L$.

IIectorea $D C$.

Hecubaca $D C$.

Hedeoma Pers.

Hedcra $L$.

Hedwigia Hook.

IIedwigia $S w$.

Hedycarya Forst.

Hedicrea Schreb.

? 293

138

302

66

187

69

185

105

129

272

185

71

140

187

187

142

201

21

215

? 94

36

225

101

176

188

22I

237

176

$2 \pi 2$
Hedychium Koen.

Fam

300

Heisteria $L$.

187

Heleninm $L$. 188

Heleocharis $R B r$. $\quad 43$

Heleochloa Host. $\quad 42$

Heleogiton Lestib. $\quad 40$

Heleophila P. B. 43

II lia Mart.

156

Helinnthemum

Tourn.

264

Helianthus $L$. $\quad 187$

Helichron Raf. $\quad \mathbf{1 8 7}$

Helichrysum Don. $\quad 185$

Helicia Lour. $\quad 113$

Helicobolus $\mathrm{W}$ allr. 4

Helicodontium

Schwaegr.

21

Heliconia $L$. $\quad \mathbf{7 5}$

Helicophyllum Brid. 21

Helicosporium Nees. 2

Ifelicotrichum Nees. 2

Helicta Cass. 187

Helicteres $L$. $\quad 295$

Heliocarpus $L$. $\quad 293$

Heliophila $L . \quad 314$

Heliopsis Pers. 187

Helio tropium $L . \quad 144$

Helleborine Pers. $\quad 78$

Helleborus $L$. $\quad 325$ 
Hemicarpha Nees. $\quad 43$

Hemichlacna Schrad. 43

Hemichroa $R B r$.

Hemicy phe Corda.

Hemidesını $R B r$.

Hemigenia $R B r$ ? 142

Hemimeris $W$. ? 148(129)

Hemionitis $L$.

26

Hemiphragma W'all. 129

Hemipilia Ldl.

Hemiragis Brid. 78

21

Hemisinapsium Brid. 21

Hemistemma Ehrb. 14:

Hemistemma Juss. 327

Hemitelia $\mathrm{RBr}$.

Hemizonia $D C$.

187

Hempelia Meyen.

Henanthus Less.

Henckelia Spr.

12

187

134

Hendecandra Eschsch. 96

Henricea Lem.

Henricia Cass.

Henriettea $D C$.

Henschelia Presl.

Hepatica Dill.

Heracleurn $\boldsymbol{L}$.

Ilerbertia Sweet.

Herculea $F$ r.

Herteria Cass.

Hericium $\boldsymbol{P}$ ers.

Ieritiera Ait.

Heritiera Michx.

Heriticra Schrk.

Ilermannia $L$.

IIermas $L$.

Ilermesia Bonpl.

Herminium $R B r$.

Hermione Salisb.

156

187

236

330

325

200

74

3

185

5

294 Hexalobus A. DC.

72 Hexanthus Lour.

71 Hexaptera Hlook.

294. Hexarrhena Presl.

200 Hexasepalum Bartl.

96 Hexisea Lall.

78 Heyderia $F r$.

69 Heylandia $D C$.

Hernodactylum $\boldsymbol{R B r}$. 71

llermupoa Löffl.

Hernandia $L$.

Herniaria $L$.

315

? 115

258

IIerobius Cass.

187

129

19

? 2

Herpetium Nees.

IEerpotrichum $\mathrm{Fr}$.

Herpysma $L d l$.

Herreria $\boldsymbol{R}$. et $\boldsymbol{P a v}$.

Herschellia Bowd.

Hertia Less.

148

187

llesperantha $\mathrm{Ker}$.

Hesperis $L$.

Hesperoscorduni Lodl. ? 66

Heynea Roxb.

Hiblertia $A n d r$.

Hibiscus $L$.

Hicorius Raf.

Hidalgoa $L c s s$.

Hidrosia $E$. M.

Hieracium $L$.

Hierochloa $\mathbf{G m}$.

Higginsia Pers.

Ililaria $\boldsymbol{H} . \boldsymbol{B} . \boldsymbol{K}$.

Hildegardia

Sch. et Endl.

Hillia Jacq.
Fam.

69) Himantia $F r$.

Fam.

? 49 Himantoglossum Spr. 78

Himantophyllum Spr. 69

Himatanthus $W$. ? 175

Hiorthia Neck. 185

Hippeastrum Herb.

69

Hippia $L$.

? 185

Hippion Spr.

156

Hippocratea $L$.

284

Hippocrepis $L$.

221

96

Hippomane $L$.

Hippomarathrum Riv.200

Hippophaë $L$.

111

236

Hippopodium Roehl. 21

Hipposeris Cass.

186

Hippotis R. et Pav.

Hiptage Gaertn.

Hippuris $L$.

Hiraea Jacq.

Hirnellia Cass.

Hirneola $F r$.

Hirpicium Cass.

176

286

34

286

185

Hirtella $L$.

Hisingera Hell.

Hispidella Barn.

Ifitchenia Wall.

Hladnikia Koch.

160

96

96

330

1Ii

314

42

175

78

306

327

296

220

187

221

188

42

176

42

Iloarea Sweet.

Hoclzelia Neck.

187

225

96

188

76

200

311

Hoffmannia $S w$.

222

176

Hoflmannseggia Cav. 222

Hohenbergia Schult. $f .56$

Hoitzia Juss.

153

Holarrhena $R B r$.

161

Holcus $L$.

42

Holigarna Roxb.

219

IIolmskioldia Retz.? 142

IIololachne Ehrb.

301

Hololepis $D C$.

185

Holonitrium Brid. 21

Holophyllum Less. 185

Holoregmia Nees. 133

Holostemma $R B r$.

Holosteum $\boldsymbol{L}$.

160

Holothrix Rich.

201

Homalanthus Ad.Juss. 96

Homalium Jacq.

241

Homalocenchrus Mieg. 42

Homalonema Schott. 36

294

176

Homanthis H. B. K. 186

Homeria V'ent.

74

Homochroma $D C . \quad 187$

Hilsenbergia Tausch. 138

Homoeanthus Bonpl. 186
Homoccladia $4 g$.

Fam.

185

Homonoin Lour. $\quad 90$

Homoplitis Triv. 42

IIonekenya Ehrh. 261

Honckenya $\mathrm{W}$. 293

Hookeria $S m$. 2I

Hopea $L$.

167

Hopea lioxb. 297

Hopkirkia Spr. $\quad 185$

Hoplophyllum DC. ? 184

Hoplotheca Nutt. 105

IIoppea Rchb. 187

Hoppea $\mathrm{W}$. 156

Horleun $L$. $\quad 42$

Horminum $L$. $\quad 142$

Furmisciun Knz. I

IIormosira Fndl. 15

Ifornemannia $W . \quad 129$

Ilornera Neck. 221

Horustedtia Retz. $\quad \boldsymbol{7 0}$

Horsfieldia $\mathrm{Bl}$. $\quad 200$

Horsfieldia $W^{\text {. }} \quad \mathbf{1 1 7}$

Hortensia Juss. 252

Hortia V'cll. 278

Hoslundia Yahl. $\quad 142$

Hosta Jacq. $\quad 138$

Hosta Tratt. 67

IIostea $W$. 160

Hostia Cass. $\quad 188$

IIottonia $L$. $\quad 126$

Houstonia $L . \quad 156$

Houttuynia Thunb. 99

Ifovea $R B r$.

Hovenia Thunb.

Hoya $R B r$.

Huanaca Cav.

Huberia $D C$.

Hubertia $A u b l$.

221

209

160

200

236

Iludsonia $L$

Ifudsonia Rob. $\quad 110$

Huernia $R B r . \quad \mathbf{1 6 0}$

Hugonia $L$ ? 294

Hugueninia Rchb. $\quad 314$

Hingelia Rchb. $\quad 200$

Humaria $\mathrm{Fr}$. 5

Humbertia Comm. $\quad 150$

Humboldtia $R$. et $P a v .78$

Humbold tia Vahl. 222

Humca $S m$.

185

Humiria DC. $\quad 306$

Humirium Rich. $\quad 306$

Humultis $L$. 
Fam.

Hunnemannia Sweet. 317

Hunteria Roxb.

Hura $L$.

Hutchinsia $\mathrm{Ag}$.

Hutchinsia $R B r$.

Hyacinthus $L$.

Hyaenanche Lamb.

Hyalostemma Wall. 330

Hybanthus Jacq. 266

Hydnocarpus Gaertn. 265

Hydnocaryon Wallr. 3

Hydnophytum Jack. 175

Hydnora Thunb.

IIydnum $L$.

Hydrangea $L$.

Hydranthelium

H. B. $K$.

Hydrastis $L$.

Hydrilla Rich.

IIydrocera $B l$.

Hydrocharis $L$.

Hydrochloa $R B r$.

Hydroclathrus Bory.

Hydrocleis Rich.

Hydrococeus $L k$.

IIydrocoryne Schwb.

Hydrocotyle $L$.

Hydrodictyon Roth.

Hydrogastrum Desv.

Hydrogeton Pcrs.

Hydroglossum $W$ illd. 24

Hydrolea $L$. 152

Hydrolinum $L k$. 1 II

Hydronystria Meyer.? 51

Hydronema Cor.

Hydropeltis Michx:

Hydrophora Tod.

Hydrophylax $\boldsymbol{L}$.

Hydrophyllum $L$.

Hydropogon Brid.

Hydropyrum $L k$.

Hydrostaclyss Pet.-Th. 98

Hydrurus $A g$.

Hygrocrocis $\mathrm{Ag}$.

Hygromitra $F r$.

Hygrophila $R B r$.

Hygroryza Nees.

Hylacium $P$. $B$.

Hylogyne Salisb.

Hylophila $L d l$.

12

321

2

175

149

21

42

11

12

42

185

113

38

Hymenacline $P$. $B$. $\quad 42$

Hymenaea $L$.

222

Hymenanthera $R B r, 266$
Fam.

Hymenatherum Cass. 187

Hymenella Fl.mex. 261

Hymenclla $\mathrm{Fr}$.

Hymenea Girev.

Hymenocallis Ilerb.

Hynienocalyx Zenk.

Ilymenocarpus Sav.

Hymenochaete $\boldsymbol{P}$. B. 43

Hymenocystis Meyer. 20

Hymenodictyon Wall. 176

Hymenogaster Vittad. 3

IIymenogyne Haw. 256

Iymenolaena $D C . \quad 200$

Hymenolepis Cass. 185

Hymenolepis Kaulf. 26

Hymenomena Cass. 188

Hymenopappus Her. 185

Hymenophallus Nees, ? 3

Hymenophyllum Sm. 26

12 Hymenophysa Meyer. 314

54 Hymenopogon $P . B .21$

42 Hymeaopogon Wall. 176

Hymenospron Spr. 221

2 Hymenostachys Bory. 26

Hymenostemum $R B r .21$

Hymenostylium Brid. 21

II ymenotomia Gaudich. 26

Hymenoxys Cass. 185

IIymenula $\mathrm{Fr}$.

Hyobanche $L$.

Hyophila Brid.

Hyoscyamus $L$.

IIyoseris $L$.

Ilyospathe Mart.

Hypaëlyptum Vahl.

Hypecoum $L$.

Hypelate $R B r$.

Hypenantron Corda.

Ilyperanthera Vahl.

Hypericum $L$.

Hyperrhiza Bosc.

Hypha Pers.

IIyphaene Gaertn.

Hyphelia Fr.

Ilyphoderma Wallr.

Hyphydra Schreb.

Hypnum $L$.

Hypobathrum $B l$.

Hypocalyptus Thunb. 221

Iy pochnaea Fr.

Hypochnus $\mathrm{Fr}$.

Hypochoeris $L$.

Hypocrea $\mathrm{Fr}$.
5

15

69

296

221

185
5

130

21

148

188

61

4ลิ

317

28!)

18

224

303

3

$? 2$

60 Juatophyllum Herb.

3 Imbricaria Comm.

5 Imbricaria $\mathrm{Fr}$.

47 Imbricaria $S m$.

21 Imhofia Herb.

175

Hypoderma Nestl.

Hypodermium $L k$.

Hypoderris $R B r$.

Hypodrya Pers.

Hypoëlytrum Rich.

Hypogaeum Pers.

Hypolaena $R B r$.

Hypolepis $P$. B.

Hypolepis Pers.

Hypolysaus Pers.

ypophialium Nees.

Hypoporum Nees.

Hypospyla $F r$.

Hypoxis $L$.

Hypoxylon Bull.

Hyptis Jacq.

Hyssopus $L$.

Hysterangium Vittad.

ysteria Reinw.

Hysterium Fr.

Ibbetsonia Sims.

Iberis $L$.

Icacina Ad.Juss.

Icacorea Aubl.

clinanthus $P$. $B$.

185

215

36

185

162

14

210

258

329

3

69

165

7

232

69

813

Impatiens $L$.

Imperata $C y r$.

3 Imperatoria $L$.

2 Impia Bluff. et $F$.

188 Incarvillea Juss.

4 Incillaria $F r$.
1 Indigofera $\boldsymbol{L}$.

Fam.

1 Inga $W$.

221

Ingenhoussia E. M. 221

45 Ingenhousia Moe.

5 et $S$.

3 Institale $\mathrm{Fr}$.

31 Intsia Pet. - Th.

? 296

3 Intybellia Cass.

222

5 Inula $L$. 187

43 Involucraria Sẹr. $\quad 195$

19 Iodes $\mathrm{Bl}$. 324

5 Ionidium Vent. $\quad 266$

43 Ionopsidium Rchb. $\quad 314$

43 Ionopsis H. B. K. 78

4 Iphiona Cass. $\quad 185$

68 Ipo Pers. 91

4 I pomeria Nutt. 153

42 Ipomoea $L$. $\quad 150$

42 Ipomopsis Mickx. 153

3 Ipsea $L d l$. 78

8 Iresine $W$. $\quad 105$

6 Iria llich. 43

4 Iriartea $R$, et Pax. 61

2 Irina $\mathrm{Bl}$. $\quad 289$

Iris $L$.

34

156

239

Iroucana Aubl.

42

Isachne $R B r$.

$\begin{array}{lr}\text { Isanthns Miehx. } & 142 \\ \text { Isaria Pers. } & 2\end{array}$

Isatis $L$.

314

Ichacmum $L$. $\quad 42$

dscharum $\mathrm{Bl}$. $\quad 36$

Isertia Schreb. $\quad 176$

Isidium $A c h$.

6

Isidorea A. Rich. $\quad \mathbf{1 7 6}$

Isidrogalvia $\boldsymbol{R}$. et $P a v .7 \mathbf{I}$

Ismelia Cass.

187

Ismene Herb.

69

Isnardia $L$. $\quad 238$

lsocarpha $R B r$. $\quad 185$

Isochilus $R_{\boldsymbol{B}}$. $\quad 78$

Isoëtes $L$.

29

Ioolepis $R_{B}$.

43

Isonema Cass. $\quad 185$

Isonema $R_{B}$. $\quad 161$

Isopetalum Eckl. et Z. 3II

42 Isoplexis Ldl. $\quad 120$

200 Isopogon $R_{B}$ r. $\quad 113$

185 Isopyrum $L$. 325

133 Isora Sch. et $F$. 295

6 Isostigma Less. 187 


\begin{tabular}{|c|c|c|c|c|c|c|c|}
\hline & Fam. & & Fam. & & Fam. & & am. \\
\hline sothecinm Brid. & 21 & Jubula Dumort. & 19 & Knappia $S w$. & 42 & Lachnanthes Filliot. & 22 \\
\hline Isotoma $R B r$ & 193 & Juglans $L$. & 220 & Knautia $\boldsymbol{L}$ & 182 & Lachnobolus Fr. & 3 \\
\hline Isotypus $K t h$. & 185 & Jürgensia Spr. & $? 291$ & Ḱnema Lour. & 117 & Lachnospermum $W$. & 185 \\
\hline Isthmia $\mathrm{Ag}$. & 11 & Juncus $L$. & 46 & Knightia $R B r$. & 113 & Lachnostoma H.B.K. & 160 \\
\hline Itea $L$ & 249 & Jungerınannia Dill. & 19 & Kniphofia Moench. & 66 & Laclınuin Rctz. & 5 \\
\hline Ittuera $\mathrm{Gm}$ & 32 & Junghansia Gm. & 210 & Knowltonia Salisb. & 325 & Lacis Schreb. & 98 \\
\hline Ityphyllus $F r$. & 23 & Jungia Gacrtn. & 232 & Knoxia $L$ & 175 & Lncistema $S w$. & 102 \\
\hline Iva $L$. & 185 & Jungia L. fil. & 186 & Kobresia Willd. & 43 & Lactuca $\boldsymbol{L}$. & 188 \\
\hline Sch.et Endl. & 294 & rus $l$. & 80 & a Roth. & 103 & Lacurus IIam. & 279 \\
\hline Ixeris & 188 & Cass. & 181 & rlinia $Z u c c$. & 274 & & 78 \\
\hline cia & 74 & $L$ & 238 & $W$ & 205 & Laclia Pcrs. & 314 \\
\hline henus Less. & 187 & dia Houst. & 96 & a Pers. & 42 & Laetia $L$ & 265 \\
\hline Fisch. & 69 & Justi & 131 & a Pall. & 188 & sia Vand. & 237 \\
\hline$B r$ & 185 & & & ra Murr. & 104 & tea Lag. & 129 \\
\hline xol & 175 & Kadsura Juss. & 324 & eria $L a x m$. & 289 & H. B.K. & 185 \\
\hline & & Cham. et Schl. & l. 176 & Ko & 100 & Ser. & 195 \\
\hline aborosa Lam. & 148 & Kaesupferia $\boldsymbol{L}$. & 76 & a Cham. & & us Nees. & 43 \\
\hline Juss. & 133 & Kageneckia $R$. et & & & 176 & a Cass. & 187 \\
\hline . & 272 & & 2227 & Kol & 71 & ia $L$. & 237 \\
\hline$r$ & 294 & a Forsk. & 185 & & 195 & & 115 \\
\hline Tall. & ? 175 & Mia Scop. & 276 & Presl. & 76 & & 200 \\
\hline Raf. & 315 & Kal & 168 & & 314 & lium M. B. & 223 \\
\hline HBr. & 21 & es Haw: & 255 & Ko & 91 & H. B. & 188 \\
\hline Thunb. & & ost. & 161 & Presl. & 296 & & 129 \\
\hline a $L$ & 128 & Raf. & 279 & & 272 & a Gaertn. & .234 \\
\hline H. B. K. & 187 & 1 & 160 & & 325 & & 296 \\
\hline Llav. & 54 & Haf. & 2178 & & 221 & mon Cass. & 184 \\
\hline$R p h$ & 232 & $B l$. & 27 & linnikovia & & & 42 \\
\hline a Hook. & 26 & Vees. & 187 & & 108 & La & \\
\hline & 96 & . & 302 & & $\% 71$ & & \\
\hline et Pav. & 42 & ec & 187 & & 188 & & 148 \\
\hline Jas & 194 & $V$ cnt. & 221 & Hoffm. & 200 & Lail & 232 \\
\hline $\boldsymbol{L}$ & 136 & m Neck. & k. 181 & ria Neck. & 222 & Moench. & 42 \\
\hline ass. & 187 & Gay. & 294 & H. B.K. & 265 & a oliv. & 14 \\
\hline L. & 96 & d. & 314 & $L$ & 185 & a $S m$ & 113 \\
\hline ers. & 185 & & 32 & tera Lam. & 221 & Lmrx. & 15 \\
\hline Bart. & 322 & & 227 & Kund & 200 & & 142 \\
\hline Sweet: & 311 & & ? 116 & & 61 & ia $A g$. & 15 \\
\hline Terr & 42 & $k$. & 40 & Kur & 227 & H.B.K. & 129 \\
\hline Pers. & 181 & Juss. & 307 & aea Fisch. & 176 & us $\mathrm{Bl}$. & 49 \\
\hline$V$ ell. & 96 & $D C$ & 236 & Ky & 294 & a $R B r$. & 13 \\
\hline$W$ & 184 & yera Mart. et & & Kyllingia L. fil. & 43 & La & 188 \\
\hline & 284 & & 299 & & & & 184 \\
\hline$R B r$. & 66 & ees. & 299 & Labe & 166 & & 22 \\
\hline & 200 & & 265 & audich. & 222 & & 303 \\
\hline & & & 46 & & 221 & P. $L$. & 162 \\
\hline Schreb. & ? 2 & & 96 & & 4 & & 187 \\
\hline & 222 & $W$ & 296 & Salisb. & 299 & a Leand. & 279 \\
\hline alisb. & 113 & & 161 & Lace & 221 & a Mart. & 118 \\
\hline & & & 243 & & 2281 & urch. & 187 \\
\hline & 232 & & 181 & a Jacq. & 66 & & 2 \\
\hline R. et $\boldsymbol{P a v}$. & 148 & & 294 & & 115 & Im $B l$. & 806 \\
\hline baea $H . L$ & 62 & Kleinia Jacq. & 185 & Lachnagrostis Trin. & $42 !$ & Lantana $L$. & 138 \\
\hline
\end{tabular}




\begin{tabular}{|c|c|c|c|c|c|}
\hline & & & & & \\
\hline apageria R. et Pav. & 61 & Lavatera $L$. & 296 & Lemna $L$ & Leptodermis Wall. \\
\hline Lapeyrousia Pourr. & 84 & avenia $S w$ & 185 & Leocarpus $L k$. & Leptodon Web. \\
\hline Lipeyrousia Thunb. & 185 & aroisicra $D C$. & 236 & Leonia R. et Pav. & Leptogium $\mathrm{Fr}$ \\
\hline ra $L a g$. & 69 & avradia Vell. & 267 & Leonotis $R B r$. & Leptoly menium \\
\hline ea Kth. & 299 & awsonia $L$ & 238 & Leontice $L$ & Schwaegr. \\
\hline Juss. & 184 & armannia Forsk. & 185 & Leoutodon $L$. & Leptolaena Pet. - Th. 298 \\
\hline go Schreb. & 42 & axmannia $R B r$. & 66 & Leontonyx Cass. $\quad 185$ & Leptomeria $R B r$. \\
\hline na $L$. & 188 & eachea Cass. & 187 & Leontophthalmum $W .187$ & Leptomitus $A g$. \\
\hline rea St. Hil. & 261 & Borsk. & 324 & podiun $R B r . \quad 185$ & Leptomon Raf. \\
\hline izabala $R$. et $P a \imath$ & 324 & Radd. & 236 & Leonurus $L$. & Leptonema Ad. Juss. \\
\hline Lari & 80 & in $L k$ & 3 & Leopoldinia Mart. & Leptopoda Nutt. \\
\hline Pers. & 255 & Gray. & 10 & Leotia Hill. & Leptopora Raf. \\
\hline ?. & 286 & Thunb. & 221 & es $S w$ & nehos Less. \\
\hline erat. & 7 & Cass. & 187 & iia $W$ & olena Presl. \\
\hline Raf. & 96 & Schrk. & 296 & cereis Trin. & num Forst. 232 \\
\hline & 5 & $\mathrm{~s}$ & 6 & za Herb. & Don. $\quad 26$ \\
\hline m $L$. & 200 & 18 Jack. & 178 & ne Lap. & m $R B r$. \\
\hline d. & 21 & Endl. & 4 & alus Lag. & $E_{n}$ \\
\hline$u r$. & 36 & ecs. & 103 & 185 & $D C$ \\
\hline$L k$ & 42 & $8 R$ & 26 & is $W$ & $d l$. \\
\hline & 236 & & 6 & us Nees. & inus $D C . \quad 185$ \\
\hline$D C$ & 187 & & 264 & 185 & Schwaegr. 21 \\
\hline P. B. & 308 & our. & 49 & 314 & A Ath. \\
\hline & 175 & & 6 & ryum Mart. & In $K n z$ \\
\hline$c c$. & I87 & & $\mathbf{2 0 0}$ & 26 & 257 \\
\hline & 3 & Rich. & 175 & llum Cass. 187 & $B r$ \\
\hline & 42 & oeffl. & 232 & in Brid. $\quad 21$ & talum Ell. \\
\hline & 294 & Schrk. & 232 & & ia $R B r$ \\
\hline ass. & 185 & Roth. & 271 & ? 187 & ? 186 \\
\hline ass. & 186 & n $D e s f$. & 2276 & erma Labill. $4 \mathbf{4}$ & 126 \\
\hline$a g$. & 186 & & 168 & P. B. & ultia $R B r$. \\
\hline Lag. & 187 & & ? 308 & $r$ & $d w$ \\
\hline ss. & 188 & & 21 & Rich. & eza Michx. \\
\hline & & & 42 & es $B l$. & tia $D C$. \\
\hline & 278 & & 201 & Ach. & gia Cham. \\
\hline hreb. & 162 & & 194 & Duf. & Bory. $\quad 15$ \\
\hline & 185 & $s_{p} r$. & 148 & $? 210$ & a Pet. $-T h .105$ \\
\hline & 185 & & 12 & a $R B r$. & Roxb. - 150 \\
\hline & 26 & Cass. & 186 & 314 & R. et Pav. \\
\hline . & 60 & & 187 & 129 & ron $R B r$ \\
\hline & 3 & 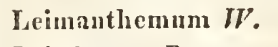 & 71 & 63 & 142 \\
\hline 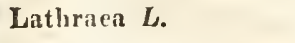 & & Pers. & 5 & a $R B r$. & 186 \\
\hline & 221 & & & 42 & ala Roxb. \\
\hline & 42 & & 23 & Cass. & e $L d l$. \\
\hline & 185 & A Pers. & 168 & ea $D C$. & schwaegr. \\
\hline & & & 6 & $D C$ & ma Meyer. \\
\hline & 188 & & & is $R B r$. & \\
\hline & 94 & Brill. & 21 & us $L k$. & $R B_{r}$ \\
\hline & 253 & umort. & 19 & lis Nutt. & Brid. \\
\hline & 15 & & 5 & us Kaulf. & es Brid. \\
\hline & 116 & & 12 & Leptochlon $P . B$. & $\operatorname{lum}$ H.R.K. 129 \\
\hline & 112 & & 257 & Jentomma Less. & Ifucophyta $R B r$. \\
\hline Lara & 391. & & & Thoncorv? & I reuconlocns vees. \\
\hline
\end{tabular}




\begin{tabular}{|c|c|c|c|c|c|c|c|}
\hline & Fam. & & & & Fam. & & $F$ \\
\hline Lencosceptrum $S m$. & 142 & Limonia $L$ & 301 & Loddigesia Sims. & 221 & Luciola Sm. & 40 \\
\hline Leucosia Pet. - Th. & 212 & Limosclla $L$. & 129 & Lodicularia $P . B$. & 42 & Luculia Su'eet. & 176 \\
\hline Lencospermum $R B r$. & 113 & Linaria Tourn. & 129 & Lodoicea Labill. & 60 & Lucuma Juss. & 165 \\
\hline Leucostemma Benth. & 261 & Linckia Lyngb. & 10 & Loefl ingia $L$. & 259 & Lucya $D C$. & 176 \\
\hline Leucostemma Don. & 185 & Linconia $L$ & 208 & Loesclia $L$ & 153 & Ludia Lam. & 265 \\
\hline Leuwenhoekia $R B r$. & 189 & Lindackeria Presl. & 265 & Logania $R B r$. & 158 & Ludolfia $W$ & 42 \\
\hline Leuzea $D C$. & 184 & Lindernia $L$ & 129) & Loiseleuria Desv. & 168 & Lndovia Poit. & 39 \\
\hline nus Schreb. & 208 & Lindleya $\boldsymbol{H} . \boldsymbol{B} . \boldsymbol{K}$. & ? 227 & Lolium $L$ & 42 & Ludwigia $L$. & 238 \\
\hline ticum Koch. & 200 & Lindleya $K t h$ & $\mathbf{2 3 9}$ & Lomandra Labill. & $? 46$ & Luffa Cav. & 195 \\
\hline Lla $v$. & 294 & Lindsaen $S m$. & 26 & Lomaria Willd. & 26 & a $\mathrm{H}$ & 293 \\
\hline teria Wall. & 178 & Linkia Cav. & 113 & Lomatia $R B r$ & 113 & Luisa Gaudich. & 78 \\
\hline$L$ & 187 & Linkia Pers. & 148 & nium $B r$. & 156 & Lumnitzcra Jacq. & 142 \\
\hline n $A d$ & 187 & ea Gron. & 178 & is Cass. & $\mathbf{I 8 8}$ & itzera $\mathrm{W}$ & 231 \\
\hline Schreb. & 185 & era Sw. & 137 & Lomatophyllum $\mathrm{W}$. & 66 & ia $L$. & 314 \\
\hline $\mathrm{Cr}$ & 200 & ris $D C$. & 187 & a Byngb. & 15 & ria Mish. & 18 \\
\hline a Desmaz. & 1 & $L$ & 310 & Lon & 185 & $\mathrm{~s} L$ & 221 \\
\hline$L e j$ & 42 & a $L$ & 221 & & 26 & ia $S p r$. & 173 \\
\hline & $\boldsymbol{7 4}$ & Liparis Rich. & 78 & Lonchocarpus H.B.K. & K. 221 & burgia & \\
\hline Lic & 225 & Lipeocercis Trin. & 42 & ma Wickst.? & $? 150$ & Hil. & 2268 \\
\hline & 3 & pha $R B r$. & 43 & Lo & 178 & a Juss. & 42 \\
\hline & 15 & eta $D C$ & 188 & s $R p h$. & 60 & $D C$ & 40 \\
\hline & & che $R B r$. & 187 & Lop & 238 & iaga $R$. et $P a v$. & \\
\hline & 200 & gis $F . M$. & 221 & as Benth. & 142 & nthus $G m$ & 262 \\
\hline$w$ & 67 & $\mathbf{L i}_{\mathbf{P}}$ & 138 & uin Brongn. & n. 42 & $\mathbf{L y}$ & 262 \\
\hline a $\mathrm{Ag}$ & 11 & $\operatorname{mbar} \boldsymbol{L}$. & 87 & $R$ ich. & 24 & cephalus $M$ & rrt. 185 \\
\hline ph. & 57 & ia Mnch. & 221 & Cer. & 72 & Mart. & 185 \\
\hline erg. & 187 & dron $L$. & 328 & ertn. & 298 & & 148 \\
\hline Cass. & 187 & Lour. & 367 & Lot & 4 & Mich. & \\
\hline & 194 & R Rchb. & 69 & derınium Chev. & 4 & Tourn. & \\
\hline reb. & 176 & us $L$ & 156 & & & on Tourn. & . 148 \\
\hline & 265 & $R B r$ & 172 & Endl. & 118 & m $L$ & 22 \\
\hline & 3 & $\operatorname{lns} R B r$ & 78 & Lophosciadium $D C$. & 200 & & 143 \\
\hline Lig & 187 & $R B r$ & 78 & mum $D_{\text {on. }}$ & 129 & Ly & 142 \\
\hline & 311 & $M$ & 221 & & 296 & & 69 \\
\hline & 200 & ireb. & 156 & & 203 & L.y & 186 \\
\hline$L$ & 137 & Lith & 42 & Lag., Less. & 187 & B. $K$. & 42 \\
\hline & 50 & Gaertn. & - 42 & & 236 & $R B r$ & 21 \\
\hline & 67 & Lith & 2259 & um Rich. & 78 & $L$ & 42 \\
\hline & 265 & in $L$. & 143 & is $D C$. & 221 & & 45 \\
\hline our. & 324 & Lito & 175 & Lo & 221 & Don. & 188 \\
\hline Lir & 78 & Litsa & 116 & a $H c r$ & 90 & & $\mathbf{2 4}$ \\
\hline ass. & 187 & Tagliab. & 56 & & 221 & & \\
\hline Lin & 6 & Ha $L$. & 122 & Lou & 96 & & ? 175 \\
\hline Lin & 96 & $R B r$ & 58 & $R B r$ & 45 & bya $\mathrm{Ag}$. & 12 \\
\hline & 138 & a Cav. & 289 & & 187 & Ell. & 160 \\
\hline $\operatorname{Lim}$ & 42 & Salisb. & 67 & a $B l$. & 26 & Lyon & 169 \\
\hline Lin & 42 & Loas: & 213 & ia Jack. & 134 & etia Less. & 185 \\
\hline & 257 & Lobadium Raf. & 217 & hyllum $E l$. & 5 & & 161 \\
\hline & 54 & Hoffm. & $\boldsymbol{\gamma}$ & Loz & ₹ 291 & us $R B r$. & 78 \\
\hline Lin & 52 & Lob & 193 & Lubi & 126 & & 88 \\
\hline loa P.B. & 43 & arpus Wight. & 330 & Lucaea $K t h$. & 42 & lie Salisb. & 113 \\
\hline Lirnnophila $R B r$. & 129 & ria Desv. & 314 & Lucilia Cass. & 185 & $L k$. & 11 \\
\hline Limodorum Tourn. & 78 & Lochnera Rchb. & 161 & Lucinaea $D C$. & 187 & $L$ & 126 \\
\hline
\end{tabular}




\begin{tabular}{|c|c|c|c|c|}
\hline & Fam. & & & \\
\hline$R B r$ & 172 & Macsa Forsk. & Marcgravia $L$ & Mattia Schult. \\
\hline sionotus Don. & 134 & Magallana Cav. & Marcliantia March. & Mattonia $R B r$. \\
\hline gsipoma II. B. K. & 193 & Nagnolia $L$. & Margaris $D C$. & Mattuschikea Schreb. \\
\hline gsurus Fr. & 3 & Magonia A. Si. Hil. & Margaritaria $L$. fll, & Iaudnytia Comm. \\
\hline \multirow[t]{2}{*}{ Lythrum $L$. } & 237 & lagydaris Kock. & Marginaria Bory. & Mauranilia Jacq. \\
\hline & & ia $L$ & Marginaria A. Rich. & Mauria $\boldsymbol{H} . \boldsymbol{B} . \boldsymbol{K}$. \\
\hline alua Forst. & 166 & Mahonia Nult. & Margyricarpus & Mauritia $B . f i l$. \\
\hline ubl. & 96 & Maluurea $A u b l$. & R. cl Pav. & Maurocenia Mill. \\
\hline nia Pet. - Th. & 73 & ia Scop. & Marialvea & ria $R$. et Pav. \\
\hline irea $D C$. & 236 & Mairia Nees. $\quad 187$ & l., Mait. & ilianea Mart. \\
\hline acanea Juss. $\quad$ ? & 302 & Majanthemum Irigg. 6t & Schrcb. & Aubl. \\
\hline acaranga $P_{e t .}-T h$. & 96 & Majeta $A u b l . \quad 236$ & nia Comm. & ea $A u b l$. \\
\hline & 142 & aila $I 1$ offm. & $? 303$ & 328 \\
\hline Im Pcrs. 2 & 221 & he Trew. & $? 150$ & nus Feuill. \\
\hline thera Nees. 1 & 187 & Malacliodendron Cav. 299 & 18 Ilall. & 129 \\
\hline ahl. & 43 & ra $L . \quad 296$ & 202 & opsis Vigicr. \\
\hline I. $B$. & 175 & Nalacochaeta Nees. & A. Rich. & Mecos \\
\hline ac & & Nalanea Aubl. & 142 & ola $L$. \\
\hline ? 3 & 837 & 78 & Sonner. & go $L$. \\
\hline & 15 & $R B r$ & $R B r$ & Medinilla Gaudich. \\
\hline & 317 & bia R. et Pav. 198 & lia Schreb. & Medusula Eschw. \\
\hline & $181 i$ & Juss. & Mar & Meesia Gacrtn. \\
\hline & 91 & Forst. & permun & Meesia Iledw. \\
\hline omm. & 279 & 221 & 46 & $D C$. \\
\hline$R B r$ & 78 & 296 & Marsypianthus Mart. 142 & ium $L d l$. \\
\hline us Lour. & 221 & 286 & Scop. $\quad 5$ & alangium Brid. \\
\hline inm Brid. & & 296 & 221 & Haw. \\
\hline & 42 & 296 & 303 & a P. B. \\
\hline$L$ & 176 & 296 & ia R.ct Pav. & $D C$ \\
\hline & 15 & 302 & 221 & s $R B$ \\
\hline Rich. & 78 & Haria Ilaw. & 186 & Schrad. \\
\hline Schreb. & 222 & 138 & 132 & 232 \\
\hline Burch. & 315 & 148 & 187 & m $L$. \\
\hline Brid. & 21 & 176 & Bert. $\quad 286$ & $L$ \\
\hline & 42 & 219 & thus Schul=: 21 & a Rich. \\
\hline RBr. $\quad 3$ & 314 & 127 & en Spr. $\quad 21$ & m Cass. \\
\hline Less. 1 & 188 & 328 & s Spr. $\quad 21$ & m $L k$ \\
\hline . B. K. 1 & 160 & na Gaertn. 302 & a R. ct Pav. 78 & Vahl. \\
\hline$F r$ & 2 & s L. $\quad 42$ & & P. Br \\
\hline Pcrs. & 150 & Gacrtn. & ra Necs. & dron $D C$ \\
\hline ium $B l$. & 78 & 221 & rus Cass. & r Corda \\
\hline Iacr & & 36 & 202 & aum F'́e. 4 \\
\hline & $2 i 8$ & 129 & m Cass. & m $I_{\text {off }} . \mathbf{2 0 0}$ \\
\hline hl. et II. & .78 & 43 & ia Llav. & DC. $\quad 222$ \\
\hline Brid. & 21 & 175 & a $s p r$ & ma Corda. 5 \\
\hline Spach. 2 & 287 & 96 & a $A u b l$. & Rohr. $\quad 187$ \\
\hline Grcv. & & Aubl. & Aubl. $\quad 160$ & n $L$ \\
\hline & 221 & $-T h$. & Hook. et A 314 & s Pohl. \\
\hline & 325 & 76 & nb., Bpl. 295 & rg. \\
\hline & 187 & ? 291 & 129 & rula Ker. \\
\hline nessa $D C$. & 187 & II.B.K. $\quad 98$ & Pcrs. & 230 \\
\hline & 187 & larattia Siv. & 187 & ania Forsk. \\
\hline Maerua 1 & 135 & arcetia $D C$. & Mattliola $R_{B} r$. & elia $L$ \\
\hline
\end{tabular}




\begin{tabular}{|c|c|c|c|c|}
\hline & & Fam. & Fam. & \\
\hline nthus $L$. & 270 & Merostachys $S p r$. & Micrelium Forsk. & Millotia Cass. \\
\hline Melica $L$ & 42 & Mertensia Humb. & Microcarpaea $R B r$. & Milnea Roxb. \\
\hline Iclichrus $R B r$. & 172 & lertensia Roth. & Microchilns Prcsl. & Nliltus Lour. \\
\hline elicocca $L$ & 289 & crtensia $W$. & Microchlaena W'all. & Mimetes Salisb. \\
\hline elicope Forst. & 288 & erulius Hall. & Microchloa $R B r$. & Nlimosa $\mathrm{L}$ \\
\hline Ielicytus Forst. & 265 & Iesanthus Nees. & Microcladia Grcv. & limnhus $L$. \\
\hline Ielidium Eschw. & 2 & Mesembryanthemum & Mierocodon $D C . f$. & sops L. \\
\hline Ieliglossus Schlchtd. & $\boldsymbol{7 1}$ & 256 & Microcoelia Lell. & um Juss. \\
\hline lotus Tourn. & 221 & Mesenterica Pcrs. & Microcoleus Desmaz. 12 & tia $L o ̈ f l$. \\
\hline P. $B$ & 42 & Icsoclastes Loll. & Microcorys $R B r . \quad 142$ & $D C$ \\
\hline In $L k$. & 42 & oia $A g$. & Mlicrocos $L$ & is $L$. \\
\hline$F r$ & 4 & 142 & Microdon Chois. & a $S m$. \\
\hline sea $L$ & 142 & Mes & Microglosea $D C$. & Mlisandra Comm. \\
\hline Melistaurum Forst.e & 239 & 116 & Microgyne Less. & Mischocarpus $\mathrm{Bl}$. \\
\hline is $L$. & 142 & 231 & Microlacna $R B r$. & drum Lks. \\
\hline Ielocactus C. Bauh. & 245 & hidia $L$. & cia Don. & \\
\hline Inna Trin. & 42 & 302 & ma $R B r$. & \\
\hline $\boldsymbol{L}$. & 294 & 176 & a $A g$. & ca Comm. \\
\hline Forst. & 162 & in Ldl. & m Bl. & \\
\hline m Lousr. & 330 & $R B r$ & Bentli. & m $\mathrm{El}$. \\
\hline g. & 11 & $R B r$ & Ldl. & \\
\hline & 195 & a $R B r$ & Mieropctalon Pers. & um Zucc. \\
\hline on $L$. & 235 & Cass. & Nicropleura Lag. & \\
\hline & 147 & a Bried. & Pal. & $\mathrm{Gm}$ \\
\hline Comm. & 96 & Lour. & 185 & acme Labill.? 1 \\
\hline & 187 & Mocnch. & Micropus $L$ & \\
\hline ct Pav. & 138 & n & Microrhynchus Less. 188 & $M i$ \\
\hline & 176 & indl. $\quad 295$ & $B l . \quad 78$ & \\
\hline & 232 & Pct. - Th. 222 & Labill. & $r e b$. \\
\hline & 20 & St. Hil. 278 & Lag. 186 & \\
\hline Bl. & 324 & Gaertn. 232 & Ad.Juss. 96 & Mniopsis $D u$ \\
\hline num $L$. & 324 & Rottb. $\quad 59$ & Don. 29! & Mnio \\
\hline Pers. & 2 & Mik. & $R B r$ & \\
\hline 1. B. K. & 129 & add. & m Less. & Mo \\
\hline$D C$ & 314 & 200 & Nutt. & \\
\hline & 142 & 187 & 105 & \\
\hline$L$ & 243 & 187 & ea Pet.-Th. 42 & rh. \\
\hline $8 \mathrm{~L}$ & 155 & 175 & 88 & \\
\hline Sn. & 169 & urum Desf. & 185 & \\
\hline P. B. & 42 & $A d$. & N. $M$. & es Mart. \\
\hline & 175 & & 5 & Juss. \\
\hline & 233 & Her. & 42 & \\
\hline orkh. & 19 & ia Dumort. & Miegia Schreb. & Mol \\
\hline Fisch. & 261 & 328 & Mielichhoferia Hornsch.21 & Mo \\
\hline \& $L$ & 96 & R. et Pav. & l1. $\quad ? 66$ & \\
\hline Ram. & $\boldsymbol{7 1}$ & 187 & 185 & \\
\hline Benth. & 142 & a Dcsf. & 42 & Molinia \\
\hline & 236 & mum Michx. 126 & Miliusa Lesch. & Mollia $G m$. \\
\hline $\operatorname{ma} L$. & 257 & a Less. & Milla Cav. & Mollia Mart. et Zucc. \\
\hline$A g$ & 11 & Chois. & 187 & Mlollia Schrk. \\
\hline ea $C a n i b$. & 260 & 218 & 188 & Mollia $W$ \\
\hline lerisma $P_{\text {ers. }}$ & 5 & Micranthus $W$ 'endl. & Millingtonia $L$. fil. & Mollinedia R. et Pav. \\
\hline Aerosporium Corda & 2 & Micrasterias $\mathbf{~ g g}$. & Mililingtonia Roxb. & \\
\hline
\end{tabular}


Topospermum Koch. 200

Molpadia Cass. $\quad 187$

Moltkia Lehm. 143

Moluccella $L$.

142

Momordica $L$.

195

Monacera Ell.

Monachne $P$. $B$.

Monactis $\boldsymbol{K}$. B. $\boldsymbol{K}$.

Monanthes Haw.

Monarda $L$.

Monarrhenus Cass. ? 187

Monathera Raf.

Monema Grev.

Moncuteles Labill. ? 187

Monerma $P$. $B$.

Monetia Her.

Monicra Aubl.

Monilia Ilill:

Monilia A. Rich.

Monilina Bory.

Monimia Pct. - Th.

Monnieria Mich.x.

Monrina R. et Pav.

Innocaryum $R B r$.

Monocera Jack.

Monoclılaena Gaudich. 26

Monochoria Presl. $\quad 63$

Monoclea Hook. $\quad 17$

Monocystis Ldl. 76

Monodonamys Pohl. 278

Monodora Dun 330

Monogramme Comm. 26

Monolophus W'all. 76

Monomeria Ldl. $\quad 78$

Monopogon Presl.

Monoporina Presl. ? 303

Monosis $D C$.

Monotoca $R B r$.

Honotris $L d l$.

Honotropa $L$.

Monotropsis $S c h w$.

Monsonia L. fil.

Monstera $A d$.

Montagnaca $D C$.

Montanoa Llav.

Montbretia $D C$.

Montezumea

Moc. et Sess.

Montia $L$.

Montinia $L$.

Moquilea Aubl.

Moquinia Spr.

Bluraea $L$.

171

311

36

187

187

74

295

257

? 238
Morchella Dill.

Morelia A. Rich.

Morella Lour.

Morelotia Gaudich.

Morenia R. et Pav.

Morettia $D C$.

Morgania $R B r$.

Moricandia $D C$.

Morina $L$.

Morinda $L$.

Moringa Burm.

Morisia Nees.

Morisonia Plum., DC.

Moronobea $\mathrm{Aubl}$.

Morus $L$.

Morysia Cass.

Moscharia Forsk.

Moscluaria R. et Pav. 186i

Moschosma Rchb.

Moschoxylon Ail .Juss. 306

Mosigia Spr.

Mougeotia $\mathrm{Ag}$.

Mougeotia Kth.

Moulinsia Camb.

Monriria Juss.

Nournucoa Aubl.

Moutouchia Aubl.

Nozinn Ort.

Mucilago Mich.

Mncor Mich.

Mucuna $A d$.

Müllera L. fil.

Münchlıausía $L$.

Mulrediun Cass.

Mulinum Pers.

IIundia $\boldsymbol{K} t \boldsymbol{t}$.

Munickia $B l$.

Muntingia $L$.

Munychia Cass.

Muraltia Neck.

225 Myag rum $L$.

193 Mycaridanthes $B l$.

54 Mycelis Cass.
Fam.

5 Mycena $\mathrm{Fr}$.

142

Fam.

176 Mycetia Reinw.

91 Mycinema $F r$.

43 Mycobanche Pers.

58 Mycoderma Pers.

314

12!)

314

182 Mycoporum Meycr.

178 Mylonotrichum Corda. 4

221 Mygalurus $L k$.

43 Myginda Jacq.

315 Mylinum Gaud.

302 Mylitta $F r$.

91 Mylocaryum $\boldsymbol{W}$.

185

142 Myogalum $L k$.

Myonima Comm.

Myoporum Bks.

186

12

294

289

? 235

150

221

96

3

\section{2}

221

221

237

188

200

292

121

293

187

272

314

301

195

197

75

91

66

176

194

186

314

78

188

Myriadenus Desv.
Myosuhilos R. et Pav.109

Myoseris $L k$.

Myosotis $L$.

Myosurus $L$.

Myoxanthus Pöpp. et E. 88

Myrcia $D C$.

Myriactis Less.

232

187

221

Myriantheia Pet. - Th. 241

Myriantlus $P$. B. ? 91

Myriaspora $D C$.

Myrica $L$.

Myricaria Desv.

Myriococcum $\mathrm{Fr}$.

Myriogyne Less.

Myrionema Grev.

Myriophyllum $L$.

Myriostoma Dese.

Myriotheca Comm.

Myristica $L$.

Myrmecia Schreb.

236

83

26!)

3

? 185

10

253

3

Myrmecodia Jack.

175

Myrobalanus Gaertn. 110

Myrodendion Schreb. 306

Myrodia $S w$.

Myrosma $L$. fil.

205

76

Myrosporium Corla.

Myrothecium Tod.

221 Neckera Hedw.

3 Nectandra Berg

3 Nectaribothrium

221 Ledeb.

311 Nectonxia H.B.K. 148

232 Needhania Cass. 187

200 Necdlamia $R B r . \quad 172$

127 Needhamia Scop. 221 


\begin{tabular}{|c|c|c|c|c|c|c|}
\hline & Fam. & & $a m$ & am. & & \\
\hline egretia $R$. et $P a v$. & 221 & Neuronia Don. & 26 & Notylia $L d l$ & Oedogonium $L k$. & 12 \\
\hline egundium Raf. & 285 & Neuraplatyceros Pluk & k. 26 & Nowodworskya Presl. 42 & & 200 \\
\hline egundo Moench. & 285 & Neuropteris Desv. & 26 & Nunnezharia $R$. et Pav. 57 & Oenocarpus Mart. & 61 \\
\hline eillia Don. & 8227 & Neurosperma Raf. & 195 & Nuphar Sibth. $\quad 319$ & Oenoplea Hedw. & 209 \\
\hline eja Don. & 187 & Neuwiedia $B l$. & 78 & Nuttallia Bart. & Oenothera $L$. & 288 \\
\hline Gaertn. & 232 & Nhandiroba Plum. & 195 & Nyctago Juss. & & 78 \\
\hline & 131 & Nicandra Adans. & 148 & Nyctanthes $L . \quad 136$ & ostylus Cass. & 185 \\
\hline nm Juss. & 320 & dra Schreb. & 150 & Nycterisition R. et Pav.165 & Ogiera Cass. & 187 \\
\hline Targ. & 14 & nia $D C$ & 221 & Nycterium Vent. $\quad 148$ & & 185 \\
\hline ray. & 4 & $\boldsymbol{L}$ & 148 & ylax Zipp. & O-lligginsia $R$. et $P a$ & v. 176 \\
\hline Pers. & 1 & lla Cass. & 187 & Nympliaea $L$. & Oidinm $L k$ & \\
\hline us Necs. & 45 & Nid & & Nyssa $L$. & un Less. & 185 \\
\hline um Desma & az. 2 & $\mathrm{Nie}$ & 315 & Nyssanthes $R B r$. & Ok & 278 \\
\hline & 11 & Nier & & & & 300 \\
\hline & & & 148 & Oberonia $L d l$. & argia Lcss. & 186 \\
\hline rmum Rich. & & $\mathrm{Nig}$ & 325 & Obione Gaertu. & Old & 176 \\
\hline ma Dietr. & & Nigri & 120 & Obojaca Cass. & & 137 \\
\hline & 12 & na Thunb. & 101 & ia $L$. & & 187 \\
\hline enes Cass. & 188 & Rich. & 78 & Ocellularia Meyer. & 01 & 26 \\
\hline d. Juss. & ร06 & Tiu & 280 & Ochna $L$ & & 187 \\
\hline Vent. & 129 & Viot & 280 & denus $D C . \quad 271$ & n Cass. & 187 \\
\hline erg. & 129 & unb. & 59 & arpus $P_{\text {ct. }}-T h .302$ & a Less. & 185 \\
\hline anthes Raf. & 210 & 8 Kaulf. & 26 & a $S w . \quad$ ? 295 & ena $R B r$. & \\
\hline & 149 & $T h$ & 241 & Juss. $\quad 163$ & ha Cass. & 185 \\
\hline & 230 & Jacq. & 221 & In Schreb. 279 & as Less. & 187 \\
\hline & 185 & g. & 13 & 314 & & 187 \\
\hline & 78 & Ilum Grev. & 14 & L. $\quad 142$ & Cass. & 185 \\
\hline$L$ & 120 & L. & 256 & Aubl., Н.В.К. 116 & n $D C$ & 21 \\
\hline & 142 & 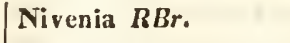 & 113 & $D C . \quad 175$ & & 209 \\
\hline Jylum RI & 58 & ent. & $\mathbf{7 4}$ & ad. $\quad 3$ & & 204 \\
\hline & 289 & Toc & 185 & Hedw. 21 & & 200 \\
\hline & 26 & c. & 12 & ill. $\quad 21$ & et Pav. & \\
\hline & 324 & H.L.K. & 266 & 175 & & 311 \\
\hline & 26 & . & 145 & 78 & & 176 \\
\hline & 6 & & $\boldsymbol{7 1}$ & Oc & 01 & 232 \\
\hline & 223 & . $L$. & 43 & 221 & & 48 \\
\hline au & 90 & l. & 175 & Kth. $\quad ? 306$ & hus Less. & 185 \\
\hline & 69 & & & a Meyr. 314 & & 21 \\
\hline & 161 & & 305 & 11 & Omi & 185 \\
\hline ks. & 175 & & 78 & a Lyngb. & tum $P \ddot{p p p}$ & 118 \\
\hline & 78 & & 137 & 129 & & 78 \\
\hline & & & 129 & 185 & ia $P . B r$. & \\
\hline ich. & 175 & $r$. & $? 1$ & H.B.K. 78 & & 96 \\
\hline & & & 10 & B. K. 185 & bium Gaertn. & \\
\hline & 187 & & 5 & Bernh. 24 & $18 P . B$. & \\
\hline F $k l$ & $\boldsymbol{7 4}$ & ent. & 137 & na Benth. 261 & On & 188 \\
\hline$R B r$ & 42 & ass. & 185 & $\mathrm{Bl} . \quad 78$ & Tourn. & 143 \\
\hline Cass. & 187 & dl. & 78 & les $L d l$. & ra Less. & 129 \\
\hline B. Juss. & 28 & & 263 & ium $L k$. & & 78 \\
\hline n $W$ eb. et $M$ & $M$. & $\mathrm{m} D C$ & 187 & L. fil. & Oncinus Lour. & \\
\hline a Desv. & & & 314 & chne $L k$. & Oneoba Forsk. & 265 \\
\hline & 187 & & 142 & caegr. 21 & Onc & \\
\hline euroloma & 314 & otochlaena $\mathbf{R B r}$. & 26 & Oedmaunia Thunb. $221 \mid$ & Oncocladiun Wallr. & \\
\hline
\end{tabular}




\section{$-80$}

\begin{tabular}{|c|c|c|c|c|c|}
\hline & & & & Fam. & 1 \\
\hline ncomyces $K l$ & 5 & Orcocallis $I R B r$. & 113 & Oryza L. & Oxyceros Lour. \\
\hline orus Brid. & 21 & Orcochloa $L k$. & 42 & Oryzopsis Michx. & Oxycoccos Tourn. \\
\hline ncorrhiza Pers. & 65 & Oreodaphne & & Osbeckia $L$ & Oxydenia Nutt. \\
\hline our. & 65 & Nees. ct $\boldsymbol{M}$. & 116 & Oscillaria Cosc. & Oxydon Less. \\
\hline in F. Nees. & $\mathbf{2}$ & Oreodoxa $\mathrm{W}$. ? & $? 61$ & scillatoria Vauch. & Oxygonum Burch. \\
\hline & 15 & Oreosclinum $I l o f f m$. & 200 & Osmanthus Lour. & Oxylobium $A n d r$. \\
\hline Gaertu. & 184 & Presigonia Schltcl. & 185 & Osmites $L$ & Oxylobus Moç. \\
\hline s Tourn. & 221 & Stackh. & 15 & psis Cass. & ris $D C$ \\
\hline & 26 & a Schreb. & 185 & iza Raf. & Oxymitra Fisch. \\
\hline & 221 & $\operatorname{um} L$ & 142 & Osmunda $L$ & Oxypetalum $R B r$. \\
\hline n $L$. & & & 113 & smundaria $L m r x$ & 106 \\
\hline Raf. & & ia Don. & 67 & orium Corda. 4 & ra $D C$. \\
\hline & 6 & $D c s v$ & 314 & leon Wallr. $\quad 130$ & $\operatorname{lma} R B r$ \\
\hline & & Hoffm. & 200 & $D C$ & a Fschw. \\
\hline m Michx. & 43 & is Cass. & 187 & neles Ldl. & yllum $B l$. \\
\hline & 38 & rpum $P . B$. & 221 & muin $L$. & $D C$ \\
\hline & & Jacks. & 221 & a Burm. & $D C$ \\
\hline ulf. & 26 & ium Salisb. & 78 & $H_{o f f m}$ & a $D C$ \\
\hline & 78 & alus Hook. & 2. 78 & in Wallr. & m $L k$. \\
\hline & 3 & s. & 66 & $\mathrm{ma} F r$ & yllum Schreb. \\
\hline & 185 & um Salisb & b. 71 & Ost & mnos $R B r$ \\
\hline & 2 & ris Bernl. & 24 & Scop. & \\
\hline & 232 & $L$ & 221 & odium Desv. & a Aubl. \\
\hline & 6 & Juss. & 289 & a Cass. & es Ldl. \\
\hline Rich. & 181 & & 138 & 78 & adren Haw. \\
\hline & 43 & & 130 & $? 109$ & s Cass. \\
\hline$r$. & 295 & $R c h b$. & 314 & um Nees. & is Less. \\
\hline & 23 & & 221 & 185 & $D C$ \\
\hline & 23 & $L$ & 37 & 128 & $B r$ \\
\hline & 23 & I Trin. & 42 & 328 & a $R B r$ \\
\hline er. & 64 & & 330 & 187 & I.B.K. 28 \\
\hline $\operatorname{cin} x$ & 26 & crt. & 185 & Pet. - Th. & ich. $\quad 221$ \\
\hline & 176 & $\ddot{o f f l . ~}$ & 258 & 311 & Michx. \\
\hline Wall. & 66 & us Nutt. & 129 & 18 & 162 \\
\hline Lour. & $11 \%$ & $R B r$ & 78 & Ldl. & Aubl. \\
\hline & $\boldsymbol{7 1}$ & um E. M. & 221 & 221 & $L$ \\
\hline & & norge & 21 & 55 & ta $L$. \\
\hline & 109 & tiunn & & ? 308 & $L$ \\
\hline & 42 & & 21 & 200 & thus Mart. \\
\hline & 78 & $R B r$ & 42 & y $R B r$ & Aubl. \\
\hline Im Eschu & & in $P . B$. & 21 & 281 & Lag. \\
\hline & 300 & ion Benth. & 142 & 129 & L. et Pav. \\
\hline & 42 & on $R B r$ & 156 & 178 & Cav. \\
\hline & 42 & Brid. & 21 & Aubl. 222 & a Cass. $\quad 185$ \\
\hline & 200 & & & andra $P$ et.-Th. 51 & ra $P$ ct. $-T h .20$ \\
\hline & 188 & & 295 & 138 & ss. $\quad 188$ \\
\hline & 179 & trichum Iledu. & 21 & 84 & ea $A u b l$. \\
\hline & 245 & enill. & 243 & 309 & bia Less. \\
\hline & 78 & nthus Sweet. & 84 & 307 & ta $R c h b$. \\
\hline & 78 & & 142 & 78 & rus Tourn. \\
\hline & 21 & hragmus & & 176 & sia Her. \\
\hline & 314 & & 314 & 124 & Pallasia $L$. \\
\hline & 43 & & & 302 & Pallenis Cass. \\
\hline
\end{tabular}




\begin{tabular}{|c|c|c|c|c|c|c|c|}
\hline & Fam. & & Fam. & & Fam. & & Fam. \\
\hline Palmaria $L k$. & 15 & Parsonsia $R B r$. & 161 & Peltophorus $P . B$. & 42 & Periphragmes & \\
\hline Palmella Lyngb. & 10 & arthenium $L$. & 187 & Peltopsis Raf. & 32 & R. et Pav. & 153 \\
\hline Palmstruckia Retz. & 129 & Pascalia Ort. & 187 & Pemphis Forst. & 237 & Periploca $L$. & 160 \\
\hline Palovea Aubl. & 222 & P'asehanthus Burch. & 198 & Penaca $L$ & 114 & Periptera $v c$ & 296 \\
\hline altonophora Kütz. & 11 & Pasithea Don. & 66 & Penicillaria Chev. & 5 & Perisporium $F r$. & \\
\hline Paludella Ehrh. & 21 & $\mathbf{m} L$ & 42 & Penicillaria $U^{\prime}$ & 42 & Peristera $D C$ & 311 \\
\hline Pamea Aubl. & 110 & Pascalia Sol. & 266 & Penicillium $L k$. & 2 & Peristera Hook. & 78 \\
\hline alea $L a g$. & 186 & Passerina $L$. & 115 & Pennantia Forst. & 96 & Peristylue $\mathrm{Bl}$. & 78 \\
\hline Cass. & 185 & ra $L$. & 197 & Pennisetum $P . B$ & 42 & na $D C$. & 315 \\
\hline Panax $L$ & 201 & $L$ & 200 & Ira $R B r$ & 172 & ia $D C$. & 200 \\
\hline via $H$ & 222 & in Schrk. & 221 & a Lehm. & 200 & ia Mart. & 222 \\
\hline $\mathrm{um} L$. & 69 & a $L$ & 148 & in Don. & 302 & tia Gandich. & 169 \\
\hline 18 L. fil. & 40 & $u b l$. & 178 & Lour. & 266 & Jachne Presl. & 42 \\
\hline n $B l$. & 265 & ev. & 4 & Pentameris P. B. & 42 & Perojoa Cav. & 172 \\
\hline n $L$. & 42 & Fr. & 4 & Pentanesna Cass. & 187 & Peromnion Schwacgr. & \\
\hline ea $L d l$. & ร8 & $x^{2}+2$ & 5 & thus Less. & 186 & - $\quad x$ & \\
\hline Feuill. & $? 91$ & $R B r$ & 84 & $8 L$ & 294 & Ait. & \\
\hline $\mathbf{P a}$ & 254 & & 176 & giua Zuce. & 160 & che Cass. & 185 \\
\hline ior. & 96 & ss. & 180 & Ion Pcrs. & 221 & ia $D C$. & 221 \\
\hline ra $\mathrm{W}^{\prime}$ & 222 & 3. $K$. & 265 & $R B r$ & 42 & a $H . B . K$. & \\
\hline $\mathbf{r} L$ & 317 & & 222 & Roxb. & 110 & facrtn. & 116 \\
\hline a Lam. & 91 & & 289 & hia Ldl. & 135 & urn. & 220 \\
\hline ckl. et Z. ? & ? 289 & & 185 & his II.B.K. & 42 & a Michx. & 185 \\
\hline Schreb. & b. 42 & $h$. & 287 & n Her. & 129 & a $S i m$. & 113 \\
\hline rsk. & 256 & $v$ & 296 & $L$ & 255 & $\mathrm{~W}$ & 306 \\
\hline & 1 & et Pav. & 94 & & 185 & ia $D C$. & \\
\hline Pct. - Th. & 43 & & 3 & Rchb. & 100 & a Ldl. & 78 \\
\hline a Cass. & 187 & Less. & 187 & um Ldl. & 76 & ium Schrad. & 188 \\
\hline$B$. & 42 & sis $D C$. & 187 & nia R. ct Pav. & .100 & na $G m$ & 221 \\
\hline Spr. & 78 & 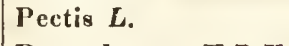 & 187 & lium $D e l$. & 129 & a Guss. & 200 \\
\hline e $B l$. & 26 & ytum R.B.K. & C. 200 & $L$ & 237 & e Don. & 185 \\
\hline & 167 & & 132 & Mut. & 96 & jis Cass. & 185 \\
\hline alisb. & 113 & Meyen. & 11 & H. B. K. & 221 & $S w$ & 235 \\
\hline & 201 & Lour. & 2289 & & 138 & emum Michx. & c. 221 \\
\hline Ker. & 74 & $L$ & 129 & Raf. & 188 & $\operatorname{ma} D C . \quad ?$ & ? 232 \\
\hline & 42 & Neck. & 96 & Don. & 26 & Tourn. & 185 \\
\hline & 90 & & 78 & & 186 & Gacrtin. & 175 \\
\hline um Juss. & 225 & $B l$. & 78 & $A u b l$. & 91 & $B r$ & 176 \\
\hline 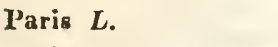 & 64 & & 277 & resl. & 42 & Jacq. & 127 \\
\hline A. St. Hil. & 296 & Cass. & 185 & & 245 & & 67 \\
\hline Aubl. & 222 & Juss. & 286 & & 186 & & 138 \\
\hline ook. & 26 & & 288 & & 160 & $L$ & 104 \\
\hline & 223 & n Her. & 311 & Trin. & 42 & & 138 \\
\hline$L$ & 222 & E. $M$. & 221 & & 2 & m $R B r$ & 185 \\
\hline & 7 & & 78 & & 185 & is $R B r$. & 314 \\
\hline uria Fée. & 6 & Andr. & $? 71$ & & $\mathbf{3}$ & a Schreb. & 225 \\
\hline & 2268 & & 19 & & 2 & ula $D C . f$ & 194 \\
\hline Ham. & 221 & & 36 & & $\boldsymbol{x}$ & a $R B r$ & 113 \\
\hline Juss. & & a Roth. & 161 & Schott. & 96 & inum $H o f f m$. & \\
\hline & 240 & & 314 & & 142 & & 176 \\
\hline & 221 & & 7 & & & Juss. & 148 \\
\hline & & & z & Peripea $A u b l$. & 129 & Peucedanum $L$. & 200 \\
\hline Parsonsia $P . B r$. & 237 & Peltodon Pohl. & 142 & Peripherostoma $G$ & & Peumus Pers. & 93 \\
\hline
\end{tabular}




\begin{tabular}{|c|c|c|c|c|c|}
\hline & Fan. & & Fam. & $\dot{x}^{-}$ & \\
\hline eziza Dill. & 5 & Phlebocarya $R B r$. & $\tau 2$ & Plyymatanthus $\mathrm{Ldl}$. & Pilocarpus Vahl. \\
\hline faffia Mart. & 105 & Phlegmatium Fr. & $? 2$ & Plyymatidium $L d l$. & Pilophora Jacq. \\
\hline Plıca $L$ & 221 & Phleogena $L k$. & 3 & Pliymatium Chev. & Philophora Uallr. \\
\hline hacelia Michx. & 149 & Phleuin $L$. & 42 & Pliysalis $L$. & Pilostyles Guill.? \\
\hline Hacidium Fr. & 4 & Phloeospora Wallr. & 1 & Physarum Pers. & Pilotrichum Palis. \\
\hline hacorrhiza Pers. & 3 & Phlomis $L$. & 142 & Physcia Fr. & la Lour. \\
\hline hacosperma Ilaw. & 257 & Phlox $L$. & 153 & Pliyscium Lour. & ea $B k s$. \\
\hline Pliaecasium Cass. & 188 & Phlyctene Endl. & 3 & Physcomitrium Brid. & Pim $_{1}$ \\
\hline Phaenocoma $D_{0 n}$. & 185 & dium Wallr. & 4 & Physedium Brill. & \\
\hline Phacocarpus & & os Lour. ? & $? 205$ & Physianthus Mart. & ia Cass. \\
\hline et Zucc. & 289 & Ploc & 58 & I'hysicarpus Poir. & 118 Léss. \\
\hline neria Ldl. & 86 & us Cass. & 188 & Physiphora Sol. & Pineda R. et Pav. \\
\hline our. & 78 & $\mathrm{Ph}$ & 139 & Physocalymna Pohl. & Ping \\
\hline$D C$ & 185 & Trin. & 42 & Pohl. & ula $L$ \\
\hline iscus Less. & 187 & in & 4 & Physocarpus Camb. & ia Oss. ? 90 (185) \\
\hline Cass. & 187 & Forst. & 67 & Physoderma Wallr. & 170 \\
\hline$B l$. & 78 & is Desv. & 26 & ium Presl. $\quad 294$ & ya Michx. \\
\hline Pers. & 66 & ll. & 231 & odium Dcsv.? $23 \%$ & Pinonia Gaudich. \\
\hline & 42 & n $\boldsymbol{L} k$. & 1 & Is Chev. $\quad 5$ & Pinus $L$ : \\
\hline & ? 185 & & 42 & Physosiphon Ldl. 78 & Pinzonia Zuccar. \\
\hline & & lum $K n$ & & Physosperinum Cuss. 200 & Piparea Aubl. \\
\hline ir. & 222 & hr & 78 & $\mathrm{Ph}$ & Piper $L$ \\
\hline$D C$ & 185 & hr & 138 & 137 & Piptatherum $P . B$. \\
\hline$L$ & 42 & & 142 & gia Benth. & haetium Presl. \\
\hline & 21 & $\boldsymbol{H}$ & 76 & In Mart. $\mathbf{3 1 5}$ & Coss. $\quad 185$ \\
\hline & 221 & Cav. & 32 & R. et Pav. 41 & Piqt \\
\hline$w$ & & & 69 & 194 & a $A u b l$. \\
\hline cnt. & 88 & & 10 & Wall. & Pir \\
\hline & & s $K n z$. & 2 & 10.1 & Aubl. \\
\hline & & & & ? 142 & 22 \\
\hline & & e Forst. & 189 & 160 & Pi: \\
\hline & 105 & & 5 & 221 & Im $L k$. \\
\hline$e v$ & 3 & & 180 & 184 & Ius Alb.ct Schw. \\
\hline s Raf. & 157 & & 96 & 3 & L. $\quad 12$ \\
\hline & 5 & & 245 & 143 & 16 \\
\hline & $18 \%$ & & 96 & 187 & 218 \\
\hline & 5 & & & 219 & 31 \\
\hline & 187 & & 175 & 289 & \\
\hline & & & 4 & 135 & $D C$. \\
\hline & 215 & $\mathrm{~m} B l$. & & in Desf. & $\mathbf{P i}$ \\
\hline & 64 & Rich. & & 188 & $27 !$ \\
\hline B. $K$. & 160 & our. & 76 & 156 & Ier. \\
\hline & 137 & & & 188 & Mart. \\
\hline icholt. & 36 & & 10 & 289 & Cass. \\
\hline & 47 & Brid. & & 266 & 200 \\
\hline & 187 & Fiseh. & 221 & 3 & $L k$. \\
\hline & 21 & & & 90 & Bks. \\
\hline & & & & 202 & 23 \\
\hline & & & 15 & 4 & $B r$. \\
\hline & $? 49$ & & 5 & um. & Pers. \\
\hline & 42 & & & 21 & In Ehrb. \\
\hline & 4 & Benth. & 142 & 28 & Pladera Rosb. \\
\hline Phichia $F r$. & 5 & um Less. & & Pilobolus Tod. & Plagianthus Forst. \\
\hline
\end{tabular}


Plagiochasma Lehm. 18

Plagiopus Brid.

Plagiotaxis Wall.

Planantlius Palis.

Plancina $F r$.

Planera $\boldsymbol{G m}$.

Plantago $L$.

Platanthera Rich.

Platanus $L$.

Platea $B l$.

Platonin Mart.

Platonia Rafin.

Platunium Juss.

Platycapnos $D C$.

Platycarpha $L$ css.

Platycarpium H.B.K. 133

Platycerium Desv. $\quad 36$

Platycheilus Cass. 18

Platychilum Dclaun.

Platycoion $D C$. $f . \quad 194$

Platygranma Meyer.

Platylepis A. Rich.

Platylobium $S \mathrm{~m}$.

Platymerium Bartl. ? 170

Platyncma

$$
\text { Wigth. et } A \text {. }
$$

Platypetalum $R B r$.

Platyporus Pers.

Platypteris II. B.K.

Platysma $B l$.

Platisma Hoffni.

Platyspermum Hoffm. 200

Platyspermum Hook. 314

Platystemon Benth.

Platystigma Benth.

Platystylis $\mathrm{Bl}$.

Platyzoma $R B r$.

Plaubelia Brid.

Plazia R. et Pav.

Plecostoina Desv.

Plectaneia Pet.- $T h$

Plectanthera Mart.? 26r

Plectranthus Her.

Plectritis $D C$.

Plectrocarpa Gill.

Plectronia $L$.

Pleea Michr.

Plenckia Raf.

Pleocarplus Don.

Pleomeles Salisb.

Pleopeltis Ilumb.

Pleroma Don.

Pleurandra Labill.
Peuridium Brid.

307 Pleuroplitis Trin.

22 Pleuropogon $R B r$.

5 Pleurospermum

88 Hoffm.

122 Pleuroatachys Brongn. 43

78 Pleurostemon Raf.

92 Pleurothallis $R B r$.

? 300 Plexaure Endl.

302 Plinia $L$. fil.

138 Plocama Ait.

138 Plocamium Lmrx.

316 Plocnglottis Bl.

Pluchea Cass.

Pluknetia Plum.

Plumaria $L k$.

$8(i$ Plumbago $L$.

21 Plumeria $L$.

Pon $L$.

6 Poarion Rchb.

78 Pocockia Scr.

221 Polalyria Lam.

Podanthun Lag.

Padaxis Desv.

286 Podaxon $F r$.

sI 4 Podisoma $L k$.

5 Podocarpus Her.

187 Podochilus $B l$.

78 Podocome Cass.

7 Podogyne Iloffmgg.

Podolobiun RBr.

Podophyllum $L$.

317 Podopterus II. B.

317 Podoria Pers.

78 Podonaemum Desi.

25 Podosperma $D C$.

21 Podosperma Labill.

186 Podosphacria $\mathrm{Knz}$.

s Podorporium Schwn.

161 Potostemon Michx.

Podostigma Ell.

142 Podostrombium Knz.

180 Potlotheca Cass.

276 Poecilodermis

175 Sch. et $\mathrm{F}$.

71 Pogonatherum $P$. $B$.

256 Pogonatum Palis.

186 Pogonia Andr.

6r Pogonia Juss.

26 Pagonopsis Presl.

236 Pogostemon Desf.

328 Pohlana Nees, ct Mart. 279

175

15

161
Fam.

42

21

42

42

Poiretia $\mathrm{Gm}$.

Poiretia I'ent.

Poitaea $D C$.

Poivrea Comm.

Fam.

Fam.

21 Polyphacum Ag. 15

Pohlia Ilerlw.

26

Polyphema Lour.

91

222 Polypliragmon Desf. 176

172 Polypodiun $L$. 26

156 Polxpogon Desv. 42

221 Poly porus $F r$. 5

221 Polypremum $L$. ? 176

234 Polypteris Nutt. $\quad 185$

78 Polanisia Raf. 315

78 Polembryum Ad. Juss. 278

232 Polcmonium $L$. 153

78

? 187

96

12

125

42

42

221

221

185

(B)

3

1

81

78

187

315

221

322

106

315

42

188

185

Polianthes $L$.

67

Polla Adans.

21

Pollalesta H. B. K. 185

Pollia Thunb.

40

143

.

Pollichin Pers.

Pollichia Sol.

Pollinia Spr.

Poloa DC.

Polyachyrus Lag.

Polyactidium $D C$.

Polyactis Less.

Polyactis $L k$.

Polyactium $D C$.

Polyalthia Bl.

Polyangium $L k$.

Polyarrhena Cass.

142

258

42

187

186

187

187

2

311

330

3

187

Polybotrya Hunb.

Polycardin Juss.

Polycarpaea Lam.

Polycarpon Löffl.

Polycenia Chois.

Polychactia Less.

Polychilos Kuhl. ct H.

Polycnemum $L$.

Polygala $L$.

Polygaster. Fr.

3 Polygonatum Desf.

2

98

160

งิ

185

lepir R. et Pav.

Polymeria $\mathrm{RBr}$.

Polymnia $L$.

26

210

258

258

140

187

78

103

272

3

64

160

106

15

230

150

187

294

Polymniastrum Lam. 187

Polymorphum Chev.

21

139

78

42

Polyodon H. B. K.

Polyosma Bl.

42

202

Polyozus Lour.

175

185

Polypappus Lessg.

Polypara Lour.

99

3 Porphyra Ag.

3

42

201

12

299

l'olystachia Hook. $\quad 78$

Polystichum Roth. 26

Polysticta Fr. 5

Polystigma DC. 4

Polystoma Gray. ' 3

Polytaenia DC. 200

Polytacnium Desv. $\quad 26$

Polythriucium Knx. 2

Polytrichum $L$. 21

Ponnaderris Labill. 209

Pamaria Cav. 222

Pomatium Gaertn. 176

Pomatoderris Schult. 209

Pomax Sol.

181

Pombalia Vand. 266

Pometia Forst. $\quad 289$

Pomunereulla $L$. 42

Pompholyx Endl. 3

Ponaea Schreb. 289

Ponceletia Pet.-Th. 42

Ponceletia $\mathbf{R B r}$.

Ponera Lull.

172

78

Pongamia Lam. 221

Pontederia $L$. $\quad 63$

Ponthieva $R B r$. 78

Pontoppidana Scop. 232

Populus $L$.

Porana $L$.

86

Porcelia R. et Pav. $\quad 330$

Poria Ilill.

Porina Ach.

Perlieria R. et Pav. 256

Porodothium Fr. 6 


\begin{tabular}{|c|c|c|c|c|c|c|}
\hline & Fam. & Fam. & & Fam. & & \\
\hline Porphyra Lour. & 138 & Prolifera Fauch. & Pteris $L$. & 26 & Purshia Spr. & \\
\hline orteoachleepia Tratt & $t .210$ & Propolis Fr. & Pterisanthes $\mathrm{Bl}$. & 308 & Puschkinia Adans. & \\
\hline ortesia Juss. & 306 & Proserpinaca $L$. & Pterinm Dosv. & 42 & Putoria Pcrs. & 175 \\
\hline ortlandia $L$. & 176 & Prosopis $L$. & Pterocarpus $L$. & 221 & Pycnanthemum & \\
\hline & 257 & rostanthera Labill. & Pterocarya Nutt. & 220 & & \\
\hline ria Jacq. & 257 & rostea Camb. & Pterocaulon Fill. & ? 187 & Pycnocephalum DC. & 18 \\
\hline ia Koen. & 32 & rosthemium $K n z$. & Pterocephalus $S p r$. & 182 & tachis Hook. & \\
\hline eria $A u b l$. & 176 & rosthesia $B l$. & hilus Hook. & 78 & & \\
\hline Aubl. & 222 & 113 & ccus Pall. & 106 & $\mathbf{P y c}$ & \\
\hline$u b l$. & 159 & m Burm. & Schrad. & 43 & ea $D c s v$ & \\
\hline et. - Th. & 116 & rotococeus $\mathrm{Ag}$. & urum $D C$. & 314 & idium Brid. & \\
\hline n $\boldsymbol{L}$ & 32 & rotomyces Ung. & Pte & 187 & Pyre & \\
\hline a $R B r$. & 42 & rotonema $\mathrm{Ag}$ & horus Cass. & 187 & Pyr & \\
\hline & 229 & aeria Turp. & hyton Cass. & 187 & & \\
\hline & 230 & 186 & Ptcropsis Desv. & 26 & iia $L k$. & \\
\hline & 36 & 142 & rmum Schrcb & b. 294 & hea Fr. & \\
\hline A. Rich. & 186 & 226 & Nutt. & 181 & $\mathbf{P y}$ & \\
\hline im. & 216 & P. B. & is $R B r$. & $\cdot 78$ & $W$ & 187 \\
\hline bl. & $? 90$ & a Comm. & P. $B r$ & 289 & & 12 \\
\hline Pav. & 56 & aeta $D C$. & a Cass. & 188 & $\mathbf{P y}$ & \\
\hline & 295 & 324 & a Pressl. & 43 & & 17( \\
\hline & 166 & Pse & & 5 & n $\mathrm{Herb}$. & 6 \\
\hline & 290 & $? 300$ & m Corr. & 297 & Py & 13 \\
\hline & 00 & E187 & ium $S w$. & & & 17 \\
\hline & 142 & 232 & Ium Bric & id. 21 & Took. & 18 \\
\hline m $R B$ & 78 & $\mathrm{ra} L k$. & & & & \\
\hline$s$. & 5 & Im Jack. & et Endl. & 294 & Py & 109 \\
\hline s. & 185 & Meyer. & Nees. & 19 & & 23 \\
\hline orda. & 18 & 2 & on. & 78 & & 23 \\
\hline & 138 & Brid. & Nutt. & 253 & & \\
\hline$L$ & 188 & 22 & Pti & 184 & & \\
\hline & 156 & in. $\quad 42$ & & & Michx. & 168 \\
\hline & 78 & sssum Llav. 78 & & ? 185 & & \\
\hline$r t$ & 144 & rpus Neck. 221 & a $A g$ & 15 & a $s$ & \\
\hline & 161 & 6 & richum Meyer. & 314 & & \\
\hline & 132 & 221 & & 105 & & \\
\hline & 150 & hus Raf. & P'til & 186 & & \\
\hline C. & 221 & & a Labill & l. 61 & & \\
\hline & 10 & 78 & & & et Pav. & 11 \\
\hline DC. & 238 & 314 & & 21 & & 29 \\
\hline L. & 126 & 175 & & 200 & & 30 \\
\hline & 210 & ertn. & & 1 & A Aubl. & 295 \\
\hline & 186 & a Kütz. & & 221 & & 28 \\
\hline ees. & 42 & pus Mart. & aertn. & 314 & & 3 \\
\hline$r b$. & 200 & & & 187 & & 6 \\
\hline Meyer. & 46 & Ecl. et Z. & & 143 & & $23 \varepsilon$ \\
\hline W'all. & 26 & Ptelen $L$ & ourn. & 325 & & 8 \\
\hline & 172 & Ptelidium Pet. - Th. 210 & & 221 & & 259 \\
\hline pus Her. & 194 & Pterandra Ad. Juss. $\mathbf{2 8 6}$ & $A c h$ & 6 & Qu & 22 \\
\hline & 138 & us Forsk. & & 15 & & 30 \\
\hline & 265 & Pterigonium $S w . \quad 21$ & & 105 & Qu & 109 \\
\hline & 91 & Pterigynandrum $H_{e d} w .21$ & & 227 & & 185 \\
\hline Proiphys Herb. & 69 & Pterilomia Kth. 142 & Purschia Raf. & 253 & Quintinia $A . D C$. & 249 \\
\hline
\end{tabular}


Quisqualis $L$.

Quivisia Juss.

Quoya Gaudich.

Racaria Aubl.

Rachiscallis $D C$.

Racomitrium Brid.

Racopilum Palis.

Racoplaca Fée.

Racoubca Aubl.

Raddia A. Rich.

Raddisia Leand.

Rademachia Thumb.

Radiola $G m$.

Radula Dumort.

Radulum $F r$.

Rafflesia $R B r$.

Rafnia Thunb.

Rajania $L$.

Ramalina Ach.

Ramaria Ilolmsk.

Ramatuclla H.B. K. 110

Ramoudia Mirb.

Ramondia Rich.

? 134

Rancagua Pöpp.ct E. 185

Randalia Petiv.

Randia Houst.

Ranunculıs $L$.

Rapanea Aubl.

Ropatea $A u b l$.

Raphanistruı Grin.

Raphanus $L$.

Raphia $P$. $B$.

Raphiolepis $\boldsymbol{L d l}$.

Raphis Lour.

Rapinia Lour.

Rapistrum Boerh.

Raputia $A$ ubl.

Raspalia Brongn.

Ratonia $D C$.

Reichelia Schreb.

Ratzeburgia $\boldsymbol{K} t h$.

Rauia Nees, ct Mert.

Rauwolfia $L$.

Ravenala $A d$.

Razoumowskia Hoffm. 203

Razumoria Spr.

Reaumuria Hasselq. $\mathbf{3 0 4}$

Rebouillia Radd.

Reboulea $K t h$.

Reecliia Moc. ct Loss. 327

Redoutea Vont.

Redowskia Cham.
18

42

$1 \% 6$

325

128

49

314

314

5!)

231

42

148

314

278

208

289

152

42

278

163

85

29

8

Rheum $L$.

Rhexia $L$.

Rhinactian $\boldsymbol{W}$.

Rhinactina Ress:

296 Rhinantlera $B l$.

314 Rhinanthus $L$.
Fam.

294

Fam.

327 Ricinus $L$.

219 Ricotia $L$.

22 Rhinopetalum Fisch. ? 67 Riedlen $V$ ent.

21 Riedleia $D C$.

188

Rhipidium Bernh.

Rhipidorlendron $W$.

Rhipidopteris Schott.

Rhipsalis Gsertn.

66 Riencourtia Cass.

26 Rindera Pall.

245 Rinorea Aubl.

299 Rhizina $F r$.

5 Ripidium Trin.

150

210

187

176

43

36

78

76

74

302

221

271

45

78

209

3

Rlyizobolu» Gaertn. 288

Rhizobotrya Tausch. 314

Rhizoctonia $D C$ :

Rhizogonia Findl.

Rhizogum Burch.

3

3

Rlizomorpha Roth.

133

Rhizonium Brid.

Rhizophora $L$.

Rhizopogon Fr.

Rhizopus Ehrb.

Rhizosperma Meyen.

Rloodiola $L$.

Ripogonum Forst.

Ritchica $R B r$.

Rittcra Schreb.

Rivina $\boldsymbol{L}$.

Rivularia Roth.

Rizoa Cav.

21

204

3

2

Robergia Schreb.

Robertia $D C$.

Robertsonia Ilaw.

Robinia $\boldsymbol{L}$.

Rbododendron $\boldsymbol{L} . \quad \mathbf{1 6 8}$

Rhodolaena Pct.-Th. 298

Rhodomela $\mathbf{A g}$.

Rluodomenia Grev.

23 Rhodonema Meyen.

209 Rlodora $L$.

Rhopala Schreb.

42

Rluacophila $B l$.

Rluse $L$.

63

Rhyuchanthera $D C . \quad \mathbf{2 3 6}$

42

Rhyncliocarpus Lcss. 187

146

Rhynchosia Lour.

Rhynchospermum

Rcinw.

188

Rlsyncliospora I'ahl.

Rhynchostylis $B l$.

210

Rhyuchotheca

103

$$
\text { R. ct Pav. }
$$

Rhytachne Ham:

8 Rhytisma Fr.

209

Riana Aubl.

Ribes $L$.

314

187

58

184

302

106

236

186

187

Riccia Mich.

Ricciella Al. Br.

Ricciocarpus Corda.

Richaeia Pet.-Th.

Rieharlia $K t$ tí.

Richardia $L$.

Richardsonia K?k.

Richea Labill.

Richea $R B r$.

? 315 Richeria Vahl.

129 Rieinocarpus Desf.
28

255

15

15

12

168

113

64

217

221

187

Robiquetia Gaudich. 78

Robsonia Berl. $\quad 244$

Rocoma Forsk. $\quad \mathbf{2 5 6}$

Roccella Ach. 9

Rocliea $D C$. $\mathbf{2 5 5}$

Rochelia Racm. et Sch. 143

Rochonia $D C$. $\quad 187$

Rodrigeuzia R. et Pav. 78

Roëlla $L$. $\quad \mathbf{1 9 4}$

Roemeria Med. $\quad 317$

Roemeria Radd. 19

Roemeria Thuıb. 127

Rocpera Ad. Juss. $\mathbf{2 7 6}$

Roeperia Spr. $\quad 96$

Roestelia Rebent. $\quad 1$

Roegeria Gay. $\mathbf{1 3 2}$

43 Rolidea Roth. 2 64

78 Rolsria Schreb. 212

Rolıria Vahl. $\quad 187$

2311 Roja Scop. 307

42 Rokejcka Forsh. 262

4 Rolandra Rotib. ? 185

266 Rollinia St. Hill. $\mathbf{3 3 0}$

244 Romanzoffia Cham. 129

16 Romulea Mar: 14

10 Ronabea Aubl. 175

16 Rondeletia $L$. 176

204 Roplostemon $B l$. 78

36 Rorida Forsk. $\mathbf{3 1 5}$

175 Foridula $L_{\text {m. }} \quad \mathbf{2 6 8}$

175 Rова $L$. 230

185 Koscoea $S m$.

172 Rosea Mart. $\mathbf{1 0 5}$

96 Rosenia Thunl. 187

96 Rosilla Lcss. $\quad 187$ 


\begin{tabular}{|c|c|c|c|c|}
\hline & & & & \\
\hline arinus $L$. & 42 & abia Colebr. & Sanicala $L$ & Saussurea $D C$. \\
\hline via Desv. & 46 & abicea Aubl. & Sanseviera Thunb. & Sallvagenia Jacq. \\
\hline ria Trin. & 42 & abinea $D C$ & Santalum $L$. & Savastana Schrk. \\
\hline$L$ & 237 & accharum $L$. & Santia Sav. & Savia Raf. \\
\hline Rothia Pers. & 221 & acciłlum Ldl. & 185 & Savia $W^{\prime}$ \\
\hline chreb. & 188 & accolabium Bdl. & Gualt. & Savignya $D C$. \\
\hline Thunb. & 176 & accoloma Kaulf. & 288 & Saxifraga $L$. \\
\hline eck. & 222 & accophurum Palis. & 284 & Scaberia Grev. \\
\hline$L$. & 42 & um F. Nees. & Jacq. & Scabiosa $L$. \\
\hline & 96 & lottis Mart. & 262 & Scaevola $L$ \\
\hline id. & 21 & dlicria Kaulf. & egmia Nces. & Scaligeria $D C$. \\
\hline Aubl. & 162 & dia $A c h$. & na Brid. & Scandis $L$ \\
\hline & 265 & ia Brongn. & 175 & Scaphis Eschw. \\
\hline & 113 & 261 & $L$ & ium Sch. et E. \\
\hline & 214 & a $L$ & R. et Pav. & liorum Ehrb. \\
\hline$D C$. & 236 & 152 & num Cass. 185 & . \\
\hline a Jon. & $? 64$ & 236 & Ldl. $\quad 78$ & $t E$ \\
\hline & 166 & $18 R p /$, & $D C$. & smus Meyen. \\
\hline all. & 142 & 59 & in $B l$. & n $K l$. \\
\hline$m m$. & 210 & 284 & n Sweet. & Scoptromyces Corda. 2 \\
\hline & 174 & Forsk. & lus Afz. & ra Jacq. ? 210 \\
\hline & $? 2$ & 169 & $R B r$ & us $P . B . \quad 42$ \\
\hline & 181 & Rich. & a Ldl. & meria \\
\hline & 229 & 103 & Lour. & \\
\hline & 187 & 81 & is Presl. & nmera $R B r$ ? $7 \mathbf{1}$ \\
\hline & 175 & 86 & 1a Fée. & Neck. \\
\hline & 221 & acis Bory. $\quad 12$ & Tet. - Th. 298 & 50 \\
\hline & 131 & ia Sch. et Endl. 295 & $R B r . \quad 160$ & a B. Rich. \\
\hline & 294 & Schreb. ? 266 & um Thunb. 221 & \\
\hline Pav. & 93 & 185 & c Sparrm. 118 & m. et Schl. \\
\hline irh. & 257 & 67 & uin Corda. & era $R c h b$. \\
\hline$B r$. & 294 & 39 & in Ehrb. & Reinw. \\
\hline & 106 & Lour. & mis Wall. & 217 \\
\hline & 187 & - H.B.K. 124 & hus Corda. & a Nees. \\
\hline & 200 & - R. ct Pav. 133 & na $R B r$ & ras Presl. \\
\hline & 26 & Mart. $\quad 236$ & a $B l$. & P. B. \\
\hline & 32 & 103 & les Presl. & in Brid. \\
\hline & 16 & 127 & 5 & pha Less. 187 \\
\hline & 64 & A.St. Hil. & n $A g$. & a Web.ot M. 21 \\
\hline & 129 & 142 & aertn. & Hium Less. 187 \\
\hline & 5 & 28 & a R. et Pav. & Kia Andrz. $\quad 314$ \\
\hline & 278 & Hüb. & 303 & \\
\hline & & a $D C$ & 318 & Michx. $\quad 324$ \\
\hline & 305 & Gaertn. $\quad 280$ & a Nees. & R. R. et Pav. 129 \\
\hline & 265 & ra L. 180 (204) & is Nees. & ton Spr. $\quad 306$ \\
\hline & & w. $\quad 127$ & 142 & rma Ehrb. \\
\hline & 175 & 179 & 78 & rma $K n \approx$. \\
\hline & 12 & ? 126 & Bosc. & gyne Cass. ? 185 \\
\hline & & 239 & 299 & laena Pet.-Th. 298 \\
\hline & 57 & R. et Pav. & 299 & loma Gaudich. 26 \\
\hline & 187 & 306 & glosmum Ldll. & nema $A g$ \\
\hline & & 317 & matum Schott. & Schizonia Pers. \\
\hline & 142 & anguisorba $L$. & Saururu" $L$. & Schizopetalon Sims. \\
\hline
\end{tabular}


Schizophyllum Fr. Fam

Schizopogon Rehb. 42

Schizostachyum Necs. 42

Schizostephanium Rchb.

Schizostoma Ehrb.

Schkuhria Roth.

Schlechtendalia Less. 186

Schlechtendalia $W, \quad 187$

Schlechtendalia Spr. 293

Sehleichera $W$. 289

Schlotheimia Brid. 21

Schmiedelia $L$. 289

Schmidtia Mnch. $\quad 188$

Schmidtia Tratt. 42

Schnella Radd. $\quad 222$

Schoenefeldia $K t h . \quad 42$

Schoenidiun Necs. 43

Schoenobiblus Mart. 115

Schoenorchis $\mathrm{Bl}$. 78

Schocnopsis Lestib. 43

Schoenoxyphium Nees. 43

Schoenus $L$. 43

Schollera Roth. 173

Schollia Jacq. $\quad 160$

Schocpfia Schreb. ? 167

Schotia Jacq. $\quad 222$

Schousboea $\boldsymbol{W}$. $\quad \mathbf{2 3 t}$

Schouwia DC. $\quad 314$

Sehrankia $W$. 223

Schrcbcra Retz. $\quad 210$

Schrebera Roxb. 133

Schubertia Bl. $\quad 200$

Schubertia Mart. 160

Schubertia Mirb. $\quad 80$

Schüblera Mart. $\quad 156$

Schultesia Mart. $\quad 156$

Seluitesia Spr. $\quad 42$

Selultzia Spr. $\quad 200$

Schwaegrichenia $s p r . \quad 72$

Schwalbea $L$. $\quad 129$

Schweiggera Mart. 302

Schweigrteria Spr. ? 291

Schweinitzia Ell. $\quad I 71$

Schweinitzia Grev. :

Schwenckia $L$.

129

Schrenkfeldia Schreb. 176

Schweykerta $\mathrm{Gm} . \quad 155$

Scilla $L$.

66

Scindaphus Schott. $\quad 36$

Sciodaphyllum P.Br. 201

Scirpidiuu Nees.

Scirpus $L$.
Fam.

Sciuris Nees. et Mart. 278

Scleranthus $L$.

Scleria Berg.

Sclerobasis Cass.

Sclerocarpus Jacq.

Sclerochaetium Nees.

Sclerochloa $P$. B.

Sclerococcum Fr.

Scleroderma Pers.

Scleroderris Pers.

Sclerodentium

Schuragr.

Sclernglossum Pers.

Selerolaena $R B r$.

Sclerolepis Cass.

Selerolepis Monn.

Scleroplyyton Fschw.

Sclerosciadium Koch. 200

Sclerostylis $\mathrm{Bl}$.

Sclerothannus $R B r .221$

Sclerotium Tod.

Sclerosylon $W$.

Scolicotrichum $K n$.

Scolochloa Mert.

Scolopacium

Eckl. et Z.

Scolopendrium Sm.

Scolosanthus Vahl.

Scolospermum Less.

Scolyuus $L$.

Scoparia $L$.

Scopolia Jacq.

8copolia $S m$.

Scopolina Schult.

Scopularia Ldl.

Scopulina Dumort.

Scorian $F r$.

Scorpiurus $I$.

Scorzonera $L$.

Scottea $R B r ., D C$.

Scouleria Hook.

Scriplida Bess.

Scrobicaria Cass.

Scrophularia $L$.

Scutellaria $L$.

Sentia Comm.

Scutula Lour.

Scybalium

Sch. et Encll.

Scyplanca Presl.

Scyphiphora Gaesto 303

Scyphiphora Gaestn. 175

Scyphofilix Pet.-Th.

43 Scyphophorus DC.
25! Scytalin E. M.

43 Seythymenis $A g$.

187 Scytoncma $A g$.

187 Scytosiphon Lyngb.

43 Scytothalia Grev.

42 Scaforthia $R B r$.

s Sebaea Sol.

3 Sebistiania Spr.

4 Sebifera Lour.

Secile $L$.

21 Sccanone $R B r$.

3 Sechum P. Br.

103 Scctidaca $L$.

185 Sectigera $D C$.

188 Scetilla Pers.

6 Sectinega Juss.

Sedúvickia Bourd.

Sedin $L$.

Scernia $R B r$.

3 Segitrella $F r$.

127 Segtria $F r$.

2 Segiera $L$.

42 Sciriun Nees.

Seiroccus Grev.

311

26

175

187

188

129

148

279

148

78 Scmerpus L. fil.

19 Sempivum $L$.

2 Senar Comm.

221 Senclra Pcir.

188 Sener $L$.

z21 Senna'oura.

21 Senrazu.

185 Senra $W$.

187 Scpedinm $L\}$.

129 Septai.

142 Scptol Fr.

209 Septorium Corda.

233 Scrapi $L$.

Sergil Gacrtn.

118 Sericospus Necs.

Sering Gay.

Scrin $S_{j} S_{y}$.

26 Seriul.

8 Seripln
Fam.

289)

221

$\mathrm{IV}^{\prime}$

10 Serissn Comm.

12 Serjania Plum.

15 Serpentinaria Gray.

15 Serpicula $L$.

61 Serpicula Roxb.

156 Serpularia Fr.

96 Serraen Spr.

116 Serratula $L$.

42 Scruria Salisb.

160 Sersalisia $R B r$.

195 Serturnera Mart.

2 次 Scsamella Rchb.

221 Sesaum $L$.

221 Sesbania Pers.

96 Sescli $L$.

16 Sesleria IIard.

Fam.

186

185

175

284

12

253

253

3

296

184

113

165

105

271

182

221

200

276

6

(i

104

I

15

140

314

200

190

Sessca R. et Pav. ? 132

Sestuchilua Kuhl. et K. 78

Sesurium L.

Setaria $\mathrm{HBr}$.

256

Scthia Kth.

42

Seutera Rchb.

283

Seymeria Purseh.

160

Shawia Forst.

129

Sheffieldia Forst.

185

Shepherdia Nutt.

? 126

Sherardia $L$.

111

174

298

26

187

? 259

219

255
274

274

314

187

222

2960

296

2

255

58

Punanthus

Pöpp. et E.

229

Sicthorpia $L$.

129

Siburatia Pet. - Tl. ? 126

Sicelinm $R B r$.

284

Sickingia $W$.

133

Sickmannia Nees.

46

Sieyos $L$.

195

296

142

2 Silaus Bess.

78

185

187

294

210

210
188

188 Silplinm $L$.

185 Silybum Siacrtir.
165

187

22y

200

262

2110

315

1.)

185

$18 \%$

184 


\begin{tabular}{|c|c|c|c|c|c|}
\hline & Fam. & & Fam. & Fam. & \\
\hline maba $A u b l$. & 280 & Solenantlıus Ledeb. & 143 & Spatellaria A.St. Hill. 266 & Sphaerotilus Kütz. \\
\hline aruba $A u b l$. & 280 & Solenarium Spr. & & Spatlıaglottis $\mathrm{Bl}$. & Sphaerozyga $\mathrm{Ag}$. \\
\hline mblocline $D C$. & 187 & Solenia $A g$. & 15 & Spaticarpa Hook. $\quad 36$ & Sphagnum Dill. \\
\hline mblum $K l$. & ? 3 & Solenia Pers. & 5 & Spathiphyllum Schott. 36 & Sphallerocarpus Bess. : \\
\hline mira $A u b l$. & 175 & Solenogyne Cass. & 185 & Spathium Lour. $\quad 99$ & Sphenella Kütz. \\
\hline msia $R B r$ & 113 & Solenostemma Ilayne. & 160 & Spathodea $P$. B. & rpus Rich. \\
\hline msia Pers. & 187 & Solidago $L$. & 187 & Spathularia Pers. & ne $R B r$ \\
\hline C $L$ & 314 & R. ct Pav. & 185 & Spathulea $F r$. & hora Kütz. \\
\hline a Aubl. & $? 302$ & Ldl. & 274 & Spathyema Raf. & Is Trin. \\
\hline a Nees. & $? 135$ & na $A c h$. & $\boldsymbol{\gamma}$ & Specklinia $L d l$. & lis $E . M$. \\
\hline Aubl. & 176 & era Hopp. & 2 efil & Specularia Heist. & Fr. \\
\hline lera Pohl. & 236 & tia Less. & 187 & Spennera Mart. & E. $M$. \\
\hline us $L$. & 138 & Sor & 188 & Spergella $R c h b$. & $\mathrm{m} H_{0 f f m}$. \\
\hline ich. & 96 & Son & 168 & 261 & Pcrs. \\
\hline & 9 & fil. & 232 & Spergularia Pers. 261 & in Med. \\
\hline Spr. & 1 & Son & 160 & Spergulastrum Michx. 261 & \\
\hline d. & 109 & $L$. & 221 & Spermacoce $L . \quad 175$ & Jacq. \\
\hline Lag. & 200 & a Pers. & 23 & Spernadictyon Roxb. 175 & Pers. \\
\hline a $F r$. & 5 & Sol & 74 & Spermatura Rchb. $\quad 200$ & Spil \\
\hline $\boldsymbol{L}$. & 314 & $L d l$. & 78 & Spermaxyrum Labill. 300 & \\
\hline n $L$ & 74 & Sop & 129 & In $P . B . \quad 43$ & a $L$ \\
\hline aertn. & 91 & Sor & 327 & lia Fr. & \\
\hline Desv. & 26 & Sor & 113 & orphia $L k$. & ha $I I, B . K$. \\
\hline 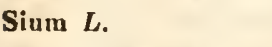 & 200 & Sor & 231 & ria Lyngb. & \\
\hline hunb. & $? 210$ & Sor & 42 & Benth. & \\
\hline rst. & 238 & $-T h$. & 215 & Sphacelia Leveill. ? 1 & Don. \\
\hline$s v$. & 64 & $R B r$ & 113 & Sphaeralcea A.St. Hill.296 & St. Hill. \\
\hline ichb. & 156 & Sor & 1 & Meyen. 11 & ich. \\
\hline & 293 & Sou & 272 & ia Ilall. 4 & \\
\hline Inia Bks. & 240 & Brongn. & 209 & iomorphium $L k .4$ & \\
\hline & & Sou & 305 & Splaaerobolus Tod. 3 & $\mathrm{~m} P$ et.-Th. \\
\hline & 2227 & alisb. & 294 & Sphaerocarpa Schum. & Schult. \\
\hline leyer. & 314 & & 66 & Sphaerocarpus Bull. $\mathbf{3}$ & \\
\hline esf. & 64 & $0 y$ & 188 & Me Mich. 16 & m Grev. \\
\hline & 64 & Juss. & 308 & a W'all.? 210 & $L$ \\
\hline $\mathrm{i}$ & 221 & iss. & 184 & P. B. $\quad 47$ & nyces Corda. \\
\hline m. & 50 & spa & 3 & us $\mathrm{Ag}$. & ma Corda. \\
\hline L. & 200 & Less. & 186 & $L k$. & Trin. \\
\hline$M . B$ & 314 & Desf. & 222 & m $S m . \quad 221$ & Spo \\
\hline Pav. & 78 & $D C$ & 176 & le Rchb. 6 & cladium Mart, : \\
\hline & 187 & a Ncck. & 232 & Fr. $\quad 4$ & cusMitsch \\
\hline rs. & 187 & & 230 & Sphaerophoron Ach. & n Lmr:x. \\
\hline$k$. & 315 & & 200 & Sphaerophysa $D C$. & ma Desmaz. \\
\hline & 187 & Lessg. & 184 & ea $A g$. & m $L k$ \\
\hline ja & 221 & & 5 & ethia Duby. & Elırb. \\
\hline la & 148 & & 34 & Sphaeropteris $\mathrm{RBr} . \quad 26$ & $R B r$ \\
\hline urr. & 296 & um $L$. & 38 & ne Wall. 306 & um Chev. \\
\hline & 148 & Spar & 185 & Sphaerosporium Schun. 1 & $A g$ \\
\hline$L$ & 126 & Thunb. & 293 & Sphaerostemma $B l . \quad 324$ & $F r$ \\
\hline Lag. & 188 & B Lk. & 221 & Sphaerotele Presl. & Lhe. \\
\hline & 266 & & 42 & Sphaerotheca Desv. & um Nees. \\
\hline & & & 221 & Sphacrotheca Cham. 129 & \\
\hline olenandra rent. & 254 & Spatalla Salisb. & 113 & Sphacrothyrium Wallr. 4 & Sporotrichum $L k$. \\
\hline
\end{tabular}




Sprekelia Herb.
Sprengelia Schult.
Sprengelia Sm.
Spumaria Pers.
Staavia Thüb.
Stacliyanthus DC.
Stachygynandrum
Palis.

Stachylidium $L k$.

Stachya $L$.

Stachytarpheta $V a h l .138$

Stackhousia Lmrx.

Stachhousia $S m$.

Stadmannia Lam.

Staehelina $L$.

Staclia Cham.

Stalagmitis Murr.

Stanhopea Hook.

Stanleya Nutt.

Stapelia $\boldsymbol{L}$.

Staphylea $\boldsymbol{L}$.

Starbia Pct. - Th.

Starkea $W$.

Statice $\boldsymbol{L}$.

Stauntonia $D C$.

Stauracanthus Lk.

Staurastrum Meyen.

Staurophora I'illd.

St aurospermum

Thonn.

Stearrhena $D_{0}$.

Stegania $\boldsymbol{R B r}$.

Stegla $F r$.

Stegia Lam.

Stegilla $R c h b$.

Stegusia Lour.

Stciractis $D C$.

Steirodiscus Less.

Stelis Sur.

Stellaria $L$.

Stellaris Moench.

Stellara $L$.

Stellulina $L k$.

Stemmacantlia Cass. 181

Stemmatosiphon

Pohl.

Stemmatospermnm P. $B$.

Steunodontia Cass.

Steminodia $L$.

Stemmona Lour.

Stammonitis Gled.

Stemmonurue $B I$.
Fant.

69

204

172

3

208

185

22

2

142

15

246

289

184

175

302

78

314

160

211

129

187

125

324

221

11

18

175

142

26

4

296

4

42

187

187

78

291

66)

115

12

2 306

42

187

129

? 64

3

109 Stilarro. $L$.

Sterculia $L$.

Steris $L$.

Stevia Cav.

Sticta Ach.

Stiftia Mik.

$\boldsymbol{R}$. et Sch. dd. Juss.

\section{Fam.}

Stemphylium Wallr.

Stenaetis Necs.

Stenanthera $R B r$.

Stenocarpus $\boldsymbol{R B r}$.

Stenochilus RBr.

Stenocladium Trin.

Stenoglossum 11. B.K. 78

Stenoglottis Lull.

Stenogyne Benth.

Stenolobium Don.

Stenomesson Herb.

Stenopetalum $R B r$.

Stenorhynchus Rich. 78

Stenostomum Gaertn. 175

Stenotaphrum Trin.

Stenotheca Mon.

42

Stephananthus Lehm. 185

Stephania Lour.

Stephania $W$.

Stephanocoma

Lessg. ? 184

324

315

Stephanoma Wallr. 3

Steplınopappus Less. 187

Stephanotis Pet. - Th. 160

Sterhuckia Schreb. 1302

Stereocaulon Ach.

Stercodon Brid.

Stereoxylun $R$. et Pav. 249

Stereum Pers.

Stcrigma $D C$.

294

8

21

Strephedium Palis.

Strephinn Schrad.

Strephodon Ser.

314 Strepsia Nuti.

Sterigmostcmon M. B. 314

Steripha Bks.

150

152

Sternbergin $W$.et Kit. 69

Sterrebeckia Lk.

Stercrin $A d$.

Stevensia Poit.

Stewartia Cav。

Stichocarpus Ag.

Stictis Pers.

Stiginanthus Lour.

Stigmarota Lour.

Stigmatanthus

\section{3}

314

176

185

299)

12

7

5

186

175

265

Streptachne HI. B. $K$.

Streptanthus Nutt.

Streptium Roxb.

Stroptochaeta Necs.

Streptogyna $P . B$.

Streptopus Michx.

Streptostachyo $P . B$.

Striaria Grev.

Striatella $\mathrm{Ag}$.

Strigula $\mathrm{Fr}$.

Stroemin Vahl.

Stromatosphaeria Gray. 4

Strombosia Bt. ? 305

Strongylium Dittm.

Strangylosperma Less. 18 \%

Strophanthus $D C$. $\quad 161$

Stigmatidium Meyer.

Stigmatopliyllon

Stigonema $A g r$.
175

\section{Strophopappas $D C$. 185}

Struchinm P. Br.

Strumaria Jacq.

286

12

95 Strithiola $L$.
Fant.

Fam.

23 Struthiopteris Willd. 2

141 Stryclinos $L$. $\quad 162$

1 Sturmia Hopp. 42

2 Simmia $R c h b . \quad 78$

96 Stylaria $A g$. 11

15 Stylidium $S w$. $\quad 189$

185 Stylimnus Raf. ? 187

185 Styloceras $A d$. Juss. $\quad 96$

42 Stylochaeton Lepr. 36

42 Stylocorgua Cav. 176

178 Styloglos8um Kühl.eth. 78

258 Styloncerua Spr. $\quad 185$

221 Stylophorum Nutt. 317

221 Stylosintlies $S w . \quad 221$

184 Stylurus Salisb. $\quad 113$

185 Stypandra $F B r$.

181 Styphelia $S m$. $\quad 172$

143 Styrax $L . \quad$ 1fi

187 Suaeda Forsk. $\quad 103$

55 Suardia Schrk. 42

232 Subularia $L . \quad 314$

232 Succisa Spr. 182

43 Succowia Merb. 311

21 Suffrenia Boll. 237

90 Suillus Mich. 5

55 Sunipia Buchan. 78

75 Suprago Gacrtu. 185

21 Surcgada Roxb.

42 Susum $B l$. ? 46

261 Sutcria $D C$. $\quad 177$

56 Sutherlandia RiBr. 221

42 Swainsonia Salisb. 221

314 Swartzia Gim. $\quad 148$

138 Swartzia $W$. 222

42 Sweetia $D C$. 221

42 Swertia $L$. 156

64 Swictenia $L$.

12 Syngrus Mart. 62

15 Eyena Schreb. 49

11 Sylithra E. M. 221

4 Symetria Bl. 2 23\%

15 Symphachne $P . B . \quad 4^{7}$

Symphionema $R B r . \quad 113$

Symphocalyx Berl. 2.44

Symphonia L. fil. $3 y^{2}$

Symphorema Rocl. ? 289

185 . 188

185 Symphysandra $D C$. 194

(i9) Sympliysia Prcsl. 173

2. S) Symphytum $L$. 1.13

175 Sympieza Licht. 16!)

115 Symplocarpus Salisb. 36 


Symplocos L.
Synandra Nutt.
Synaphea RBr.
Synarthrum Cass.
Synassa Ldl.
Syncarpha DC.
Synchodendron Boj.
Syncoelinm Wallr.
Syncollosia Ag.
Syndonisce Corda.
Synedrclla Gacrtn.
Syngoninn Schott.
Synoum Ad. Juss.
Syntherisma Walt.
Syntricha W'd. et M.
Synzygantliera
P. ct Pav.

Syrenia Andrz.

Syringa $L$.

Syringasma Mart.

Syrrhepodon Schwaegr. 21

Sytrepha Burch. ? 161

Systylium Hlornsch.

Syzygites E/hrb.

Syzygium Gaertn.

Tabernaemonta $\boldsymbol{L}$.

Tacea Forst.

Tachia Aubl.

Tachia Pers.

Tachibota Aubl.

Tachigalia $\mathrm{Aubl}$.

Tacsonia Juss.

Taenitis Su;

Taeniocarpum Desv.

Tacuiophyllum Bl.

Tafalla R. ct Pav.

Tagetes $L$.

Tainia $B l$.

Talasium Spr.

Talauma Juss.

Taliera Mart.

Taligalea Aubl,

Talinun $A d$.

Talisia Aubl.

Tamarindus $L$.

Tamarix $L$.

Tambourissa Sonn.

Tamnus Juss.

Tanonia Aubl.

Tamus $L$.

Tanacetum $L$.

Tanaëcium $S w$.

161

129
Fam.

167

142

113

187

78

185

$18 \AA$

22

1

18

188

36

306 Taraxacum Hall.

42 Tarchonanthus $L$.

21 Taresna Gaertn.

Targionia Mich.

96 Tarrietia $B l$.

314 Tarmannia $\mathbf{R B r}$.

137 Táuscheria Fisch.

Tavaria $R$. et Pav.

Taverniera $D C$.

Taxanthema Neck.

21 Taxodium Rich.

2 Taxus $L$.

232 Tayloria $l l o o k$.

Tecmarsis $D C$.

161 Tecona Juss.

80 Tectona $L$. fil.

156 Teedia Rud.

222 Teesualia $R B r$.

2 2fi6 Teganium Lehm.

222 Tegularia Rcinw.

198 Telekia Baumg.

26) Teleozoma $\mathrm{RBr}$.

221 Telephium $L$.

78 Telfairia Ilook.

101 Telina $\boldsymbol{E} . \boldsymbol{M}$.

187 Telipogen II. B. K.

78 Tellima $R B$ r.

42 Tclopea $R B r$.

328 Temachium Wallr.

58 Templetonia $R B r$.

138 Temus Mol.

$25 \%$ Tenoria Spr.

289 Tepesia Gaertn.

222 Tephrosia Pcrs.

269 Terronnus $P . B r$.

93 Terebinthus Juss.

? 65 Terminalia $L$.

138 Ternatea $K t h$.

? 65 Ternstroemia Mu\%

? 185 Terpmanthus

Nees, et Mart.
Fam.

163 Tertrea $D C$.

Fam.

110

38

Tessaria R. ct Pav.? 187

Tessarthronia Turp.

Tessiera $D C$.

Testicularia $\mathrm{Kl}$.

Testudinaria Salisb.

Teta Roxb.

? 215 Tetracera $L$.

177 Tetracmis Brid.

212 Tetractis Spr.

222 Tetradenia Benth.

221 Tetradenia Nees.

188

185

176

Ii

3. 286

329

314

315

221

125

80

81

21

185

133

138

129)

314

145

26

187

26

258

195

221

78

78

248

113

11

Teucrium $L$

329 Thulamia Spr.

200 Thalassia Banks.

176 Thalia $L$.

221 Thalictrum $L$.

221 Thamnea Sol.

218 Thamnia $R B r$.

110 Thamnidium $L k$.

221 Thamnochortus $\boldsymbol{B}$ erg. 45

299

Thamnomyces Ehrb.

Thamnophora $\mathrm{Ag}$.

11

175

3

? 81

327

21

335

142

116

214

215

187

314

325

208

265

2

45

4

15

278 Thauatophytum Nees.
Thapsia $L$.

Fam.

Thaspium Nutt.

200

200

Thaumasia Ag.

15

299

65

Thea $L$.

96

115

138

Theka Jusy.

? 125

Thelactis Mart.

Thalasis $\mathrm{Bl}$.

\section{2}

78

3

Thelebolus Tod.

Thelephora Ehrh.

Thelopogon Roth.

? 188

Thelotrema Ach. 6

25f Thelychiton Endl. 78

Thelygonum $L$. 103

Thelymitra Forst. 78

89 Thelyra Pet. - Th. 225

Thelythannos Spr.f. 187

8 Thenardia $H$. B. K. 161

Theobroma Juss. 294

16 Thermia Nutt. 221

285 Thermopsis $R_{B r}$. 221

187 Therogeron $D C . \quad 18 \%$

78 Thesium $L$. 109

21 Thespesia Corr. 296

42 Thevetia Juss. $\quad 160$

14 Thibaudia $R$. et $P$ av. 173

286 Thlaspi $L$. $\quad 314$

Thoa Aubl. ? 81

Thomasia Gay. 294

43 Thompsonia $R_{B r} . \quad 197$

42 Thorea $\mathrm{Ag}$. $\quad 12$

14 Thottea Rottb. 121

283 Thouinia Poit. 289

236 Thouinia $S m . \quad 150$

Thrasia $I I . B . K . \quad 42$

21 Threlkeldia $\mathrm{RBr} . \quad 103$

187 Thrinax $L$. fil. 57

50 Thrincia Roth. 188

142 Thrixspermum Lour. 78

81 Thrombium Wall. 6

32 Thryallis Mart. 286

76 Thryocephalon Foest. 43

1

2

(n)

2

88

Thuarea Pers. $\quad 42$

Thuja $L$. $\quad 80$

Thunbergia L. fil. 131

Thyana Ham. $\quad 289$

Thylachinm Lour. $\quad 315$

Thymbra $L$. $\quad 142$

Thymelina Hffmgg. 115

Thymophylla Lag. 187

.

.

95

1

9

3

.

4




\begin{tabular}{|c|c|c|c|c|c|c|c|}
\hline Thymus $L$. & $\begin{array}{l}\text { Fain. } \\
142\end{array}$ & Toresia R. et $P a v$ & $\begin{array}{r}\text { Fum. } \\
42\end{array}$ & Trentepolulia Roth. & $\begin{aligned} \text { Fum. } \\
\text { S14 }\end{aligned}$ & Trichophyllum Nutt. & $\begin{array}{l}\text { Fam. } \\
18 \gamma\end{array}$ \\
\hline Thyreanthus Ell. & 221 & Toricellia $D C$ & 201 & Trepocarpus Nutt. & 200 & Trichopilia Lall. & 78 \\
\hline Tloyrsanthus Schk. & 126 & Torilis Spr. & 200 & Trepposa $L k$. & 14 & Trichopterya Necs. & 42 \\
\hline hyrsopteris Knz. & 26 & entilla $L$. & 229 & Trevirania & 129 & & 195 \\
\hline Thyвanucarpus Hook. & 314 & Torreya $S_{p} p$. & 124 & Triaclıne Cass. & 186 & & \\
\hline Thysanulaena Nees. & 42 & Tortula Healw. & 21 & Triadeniutn Raf. & 303 & Sch. et Endl. & 294 \\
\hline jitrion & & Tortula $W$ & 138 & T'riadica Lour. & 96 & Trichospermum $B l$. & 205 \\
\hline & 21 & Torula Pers. & 1 & Triaena $I l . B . K$. & 42 & Trichospira $U$. H. K. & 185 \\
\hline tus $R B r$. & 66 & Torulinium Desv. & 43 & Trianoptiles Fenzl. & 43 & Trichneporum Don. & 134 \\
\hline num IIoffm. & 200 & Cosagris $P . B$. & 42 & Triantlua Nutt. & 81 & mua Cass. & 187 \\
\hline$L$. & 218 & $u b l$. & 289 & ma Sauv. & 256 & Tri & 142 \\
\hline Lehm. & 144 & & 222 & & 78 & Corda. & \\
\hline ina $A u b l$. & 236 & $L$. & 146 & ra Desv. & 42 & in Iledw. & . 21 \\
\hline Aubl. & 278 & omb. & 133 & nma $R B r$. & 236 & Ha Lehm & ง. 229 \\
\hline nnia $D$ & 200 & & 302 & $\mathrm{Fr}$ & 4 & $L k$. & \\
\hline$A u b l$. & 328 & Tox & & li ia Ldl. & 78 & Tri & 88 \\
\hline Pursh. & 227 & & 289 & s Tourn. & 276 & & 2 \\
\hline luss. & 74 & Toxicodenilron Thunb. & 3. 96 & ia A. Rich. & 175 & & 286 \\
\hline eyer. & 187 & av. & 42 & yum Lour. & 96 & alisb. & 113 \\
\hline il & 293 & 0 & 129 & in $D C$. & 236 & & Giti \\
\hline ril & 321 & $n L$. & 194 & Schreb. & 96 & & 124 \\
\hline$i c h$. & 255 & & 42 & Triceraja $\mathrm{W}$. & 284 & & \\
\hline$L$ & 56 & Michx. & 42 & Andr. & 314 & & 292 \\
\hline lerlw. & 21 & Fr. & 6 & eta $P . B$ & 42 & Tri & 42 \\
\hline$R p h$. & 175 & Brid. & 21 & $t_{c h}{ }^{3}$ Fhrbg. & 276 & $\mathbf{T r}$ & 124 \\
\hline$S$ & 28!) & indg. & 200 & "ée. & & & 71 \\
\hline Chce. & 3 & chb. & 42 & lis Lestib. & 43 & & 157 \\
\hline Nutt. & 78 & Tra & .200 & chrad. & $18 ?$ & & 42 \\
\hline & 201 & Brid. & 21 & all. & 3 & s Lour. & 96 \\
\hline arll. & 78 & & 42 & & 306 & $L$ & 126 \\
\hline esf. & 187 & un $L k$. & 200 & $R B r$ & 105 & & 221 \\
\hline & 208 & C. & 327 & Presl. & 26 & & 50 \\
\hline$I$ & 129 & Tri & 49 & & 324 & 11 Fisch. & 42 \\
\hline h. & 22 & Tra & 103 & us $\mathrm{IV}$. & 293 & $L$ & 221 \\
\hline esv. & 289 & Tras & 96 & & & Tri & 284 \\
\hline & 36 & & 200 & $P \ddot{u}$ & 78 & m Lall. & 78 \\
\hline Aubl. & 176 & Less. & 187 & Tri & & $\mathbf{T r}$ & 289 \\
\hline illt. & 24 & B. $K$. & 187 & & 209 & & \\
\hline & 289 & & 188 & 8 II. B. K. & & & 187 \\
\hline lluds. & 71 & I. $B$ & 106 & $D C$ & 42 & Trig & 145 \\
\hline rtn. & & T'ourn. & 200 & 28 Pers. & 205 & chlchtd. & 330 \\
\hline$L$ & & & 210 & Cass. & 186 & & 43 \\
\hline hunb. & 116 & Tri & 253 & Pers. & 3 & & 185 \\
\hline & 299 & & 43 & a $R B r$ & 143 & & 293 \\
\hline & 7 & & 185 & iun Chev. & & Tri & 61 \\
\hline chreb. & 284 & Tra & & Michx. & & es Cass. & 187 \\
\hline uss. & & & 26 & is $B l$. & 88 & aertn. & 186 \\
\hline & & & 273 & Less. & 185 & & 200 \\
\hline $\mathrm{Fr}$ & $? 2$ & & 21 & eq $L$. & 26 & & 15 \\
\hline Aubl. & 36 & & 236 & inum Rchb. & 21 & Tric & 43 \\
\hline & & & 5 & Ker. & 74 & Trie & 42 \\
\hline & 200 & Trentepoli & 21 & LIll. & 60 & & 175 \\
\hline & 129 & |'Trentepohlia Mart. & & Trichophorum Rich. & $\$ 31$ & Trionychon W'allr. & 130 \\
\hline
\end{tabular}




\begin{tabular}{|c|c|c|c|c|c|c|c|}
\hline & Fam. & & Fam. & & Fam. & & Fam. \\
\hline Triopteris $L$. & 286 & Trypethelium Spr. & & Ulota Mohr. & 21 & Valentiana Rof. & 178 \\
\hline Triosteum $L$ & 178 & Tryphiia Ldl. & 78 & Ulothrix Kütz. & 12 & Valentinia $S w$. & 289 \\
\hline Triphaca Lour. & 294 & Tschudya $D C$. & 236 & Ulva Lmrx. & 14 & Valenzuelia Bert. & 289 \\
\hline Triphasia Lour. & 301 & Tuber Mich. & 3 & Ulvastrum $D C$ & 14 & Valeriana $L$ & 180 \\
\hline Triphragmium $L k$. & 1 & Tubercularia Tode. & 1 & Umbilicaria $H_{0 f f m}$. & 7 & Valerianella Mnch. & 180 \\
\hline Tripinna Lour. & 129 & Tubilium Cass. ? & 185 & Umbilicus $D C$. & 255 & Vallarie $R B r$ & 161 \\
\hline Tripinnaria Pers. & 129 & Tubulifera $\mathrm{Gm}$. & 3 & Uncaria Schreb. & 178 & Vallea Mut. & 202 \\
\hline Triplaris $L$ & 106 & Tubulina Pers. & 3 & Uncinia Pcrs. & 43 & Vallesia $R$. et Pav. & 163 \\
\hline Triplasia $P . B$. & 42 & Tuburcinia $F r$. & 2 & Undina $F r$. & 10 & Vallifilix Pet.-Th. & $2 t$ \\
\hline Triplenra $L d l$. & 78 & Tula del. & 176 & Ungeria Sch. et E. & 294 & Vallisneria $L$ & 53 \\
\hline Triplima Raf. & 43 & Tulbaghia $L$. & 66 & Uniola $L$. & 42 & Vallota Herb. & 69 \\
\hline Triplatein Bartl. & 261 & Tulipa $L$. & 67 & Unisema Raf. & $6 \hat{3}$ & Valonia $A g$. & 14 \\
\hline Triplostegia $\mathrm{W}^{\prime}$ all. & 180 & Tulostoma Pers. & 3 & Unona $L$. & 330 & Valsa Aduns. & 4 \\
\hline Tripogon Roth. & 42 & Tunica Scop., Koch. & 262 & Unxia $F$ & 187 & Vancouveria Morr. & 323 \\
\hline Tripolium Nees. & 188 & Tupistra Ker. & $26 ; 4$ & Urachne $P . B$, & 42 & Vanda $R l R$. & 78 \\
\hline Tripяаси m $L$ & 42 & 'Turbinaria $L m r x$. & 15 & Uralepis Nutt. & 42 & Vandellia $P . R r$. & 129 \\
\hline Tripterella Michx. & 73 & Turczaninoria $D C$. & 187 & Urania Schreb. & 75 & eria Comin. & 175 \\
\hline Tripteris Lessg. & 187 & Turgenia $H_{o f f m}$. & 200 & Uraria Desv. & 221 & Vanhallia March. & $\hat{\mathbf{3}}$ \\
\hline Tripterocarpus & & Turgosea Haw. & 255 & Uraspermum Nutt. & 200 & Fanieria Lour. & 91 \\
\hline Meissn. & 289 & Turia Forsk. & 195 & Urceola Roxb. & 161 & Vanilla $S w$ & 78 \\
\hline Triptilion $R$. et Pav. & 187 & Turnera $L$ & 242 & Urceolaria $A c h$. & 6 & Vanillosma Lcss. & 185 \\
\hline Trirrhaphis $R B r$ & 42 & Turpinia $H . B$. & 184 & Urceoliua $R c h b$. & 60 & Vareca Gaertn. & 197 \\
\hline Trisetaria Forsk. & 42 & Turpinia Pers. & 221 & Uredinaria Chev. & -1 & Varennea $D C$. & 221 \\
\hline Trisetum Pers. & 42 & 'Turpinia Raf. & 217 & Uredo Pers. & 1 & Vargasia Bert. & 286 \\
\hline Tristachya Nees. & 42 & Turpinia $V$ ent. & 211 & Urene $L$ & 206 & Vargasia $D C$. & 187 \\
\hline Triotagma Poepp. & (i8 & Turraea $L$ & 306 & Urginea Steinh: & 66 & aria $A c h$. & 6 \\
\hline Tristania $R B r$ & 232 & Turritis $L$ & 314 & Urochloa $P . B$. & 42 & a $L$ & 147 \\
\hline Tristegis Noes. & 42 & Tursonia Cass. & 185 & Uromyces $L k$. & 1 & Varthemia $D C$. & 185 \\
\hline Tristellateia $P_{e t}-T h$. & .286 & Tussilago Tourn. & 187 & Uropetalum Ker. & 66 & Vascoa $D C$ & 221 \\
\hline Tristemma Juss. & 236 & Tylloma Don. & 186 & Urophyllum & & Vatuirea $A u b l$. & 223 \\
\hline Triteleia Hook. & 67 & Tylophora $R B r$. & 160 & Juck. et $W$ & 176 & a $L$. & 298 \\
\hline Triticum $L$. & 42 & Tylostylis $B l$. & 78 & Urospermum Scop. & 188 & a $L$ & 297 \\
\hline Tritoma $K e r$ & 60 & Tyumpanis Tod. & 4 & Ursinia Gaertn. & 187 & Vauanthes Ilaw. & 255 \\
\hline Tritomanthe Hffmgg & g. 66 & Typha $L$. & 38 & Urtica $L$ & 90 & Vaucleria $D C$. & 14 \\
\hline Tritonia Ker. & $7+$ & Typhoderma Gray. & 12 & Urvillea $H . B . K$. & 289 & Vauquelinia Corr. ? & 227 \\
\hline fetta $L$. & 293 & Typbodium Lk. & 4 & Unnea $H_{\text {off } m}$ & 9) & Vauthiera $A$. Rich. & 48 \\
\hline Trixago $L k$. & 129 & Typhuides Mocnch. & 42 & Ustalia Fr. & 6 & Velaea $D C$ & 200 \\
\hline Trixis Mitch: & 253 & Typhonillu Schott. & 36 & Usteria Cav. & 129 & Velaga Gaertn. & 294 \\
\hline Trixis $P . B r$. & 186 & 'Typhula Br. & 5 & Usteria Lam. & 158 & Velezia $L$ & 262 \\
\hline Trixis $S w$ & 185 & Tyrimnus Cass. & 181 & o $L k$. & 1 & Vella $L$. & 314 \\
\hline Trizenixis Lall. & 78 & Tytonia Don, & 312 & Utricularia $L$. & 128 & Veileja $S m$. & 190 \\
\hline Trochern Rich. & 42 & & & $L$ & 330 & Vellosia Vand. & 72 \\
\hline Trochetia $D C$ & 294 & Ucacea Cass. & 187 & Uvedalia $R B r$. & 129 & Veltheimia Gled. & 66 \\
\hline Trochiacanthes Koch. & 200 & Veriana $W$ & 176 & Uvularia $L$. & $? 71$ & Venidium Less, & 187 \\
\hline Trochocarpa $R B r$. & 172 & Udora Nutt. & 53 & & & Ventenatia Cav. & 172 \\
\hline Trollius $L$ & 325 & Ugena Cav. & 24 & Vaccaria Dod. & 262 & Ventenatia $P$. $B$. & 299 \\
\hline Trommadorffia Mart. & 105 & Ulantha Hook. & 78 & Vaccinium $\boldsymbol{L}$ & 173 & Ventenatia $S m$ & 189 \\
\hline Tropaeolum $L$ & 290 & Ulex $L$ & 221 & Vaginaria Bory. & 12 & Ventilago Gaertn. & 209 \\
\hline Trophis $L$ & 90 & Ulloa Pers. & 148 & Vahea Lam. & $16 i 1$ & Vepris Comm. & 279 \\
\hline Tropidia $L d l$ & 78 & Ullucn Loz. & 257 & Valılia Thunb. & 218 & Veratrum $L$ & 71 \\
\hline Tropidolepis Tausch. & 187 & Ulunaria Camb. & 227 & Vaillantia $D C$. & 174 & Verbatcum L. ? 148 & (129) \\
\hline Troximon Don. & 188 & Uluns' $L$. & 88 & Valantia $L$ & 174 & Verbena $L$ & 138 \\
\hline Truncaria $D C$ & 286 & Ulospermum $L k$. & 200 & Valdesia $R$. et Pav. & 236 & Verbesina $L_{:}$ & 187 \\
\hline
\end{tabular}


Verea $W$.

Vermieularia Tod.

Vermontea Comm.

Vernicia Lour.

Vernonia Schrcb.

Veronica $L$.

Verpa $S w$.

Verrucaria Pers.

Verticillaria

$\boldsymbol{R}$. et Pav.

Verticillium Nees.

Verticordia $D C$.

Vevicaria Lam.

Verillaria Hoffmgg.

Viborgia $S p r$.

Viborquia Ort.

Vibrissea $F r$.

Viburnum $L$.

Vicatia $D C$.

Vicia $L$.

Vicoa Cass.

Vieusseuxia Lar.

Vigna Sav.

Vignea $P$. $B$.

Viguiera $I I . B . K$.

Vilfa Adans.

Villanova Lag.

Villarsia Tent.

Vilınorinia $D C$.

Viminaria $S m$.

Viuca $L$.

Vincentia Boyer.

Vincentia Gaudich.

Vincetoxicum Pers.

Viola $L$.

Virea Gaertn.

Virecta L. fil.

Virgaria Nees.

Virgilia Lam.

Virgularia $R$. et Pav. 120

Virola Aubl.

Viscum $L$.

Visenia Houtt.

Vismia Vand.

Vitex $L$.

Vitis $L$.

Vitmannia Turr.

Vittadinia Rich.

Vittaria Sm.

Vittmannia $V a h l$.

Vleckia Raf.

Voacanga Pet. - Th.

Voandzeia Pet. $-T h$.
Fam.

255 Vochya Vaud.

4 Vochysia Juss.

241 Vogelia $\mathrm{Gm}$.

96 Vogelia Lam.

185 Vohiria Lam.

129 Voigtia Roth.

5 Voigtia Spr.

6 Voitia Hornsch.

Volkumeria L.

302 Volkmannia Jacq.

2 Volubilaria Lmrx.

932 Volutella Tod.

314 Vouacapoua Aubl.

221 Vouapa Aubl.

221 Votomita Aubl.

221 Voyra Aubl.

5 Vrolikia Syr.

179 Vulpia $\mathrm{Gm}$.

200

221 Wachendorfia $L$. 272

187 Wahlbomia Thunb. 327

74 Wahlenbergia Schrad. 191

221

43

187

42

187

155

221

221

161

293

43

160

266

188

176

2

221

129

117

203

294

303

138

308

124

187

26

280 Wendlandia $W$.

142 Wcultia Meycn.

163 Wensea Wendl.

221 Werneria $K t h$.

291

ริอ

156

188

21

5 (1)

? 185

281

Walkera Schreb.

185

176

187

129

74

204

251

21

56

176

324

? 276

$1 \pm 2$
Fam.

291

125

Wiborgia Thunb.

184 Wigandia Neck.

138

138

15

221

222

202

156

129

42

Wigandia $R$. et Pav. 15

Wikstroemia $S p r$.

Willdenowa Cav.

Willdenowia $\mathrm{Gm}$.

Willdenowia Thunb.

Willemetia Brongn.

Willemetia Neck.

Willichia Mut.

Willughbeia Scop.

Wilsonia $R B r$.

Windınannia $\boldsymbol{P} . B r$.

Windsoria Nutt.

Wintera Murr.

Winterana $L$.

Wisteria Nutt.

Witharia Pauq.

Witheringia Her.

Witsenia $L$.

Wittelsbachia Mart. 299

Wollastonia $D C$.

Woodfordia Salisb.

Woodsia RBr.

Woodvillea $D C$.

Woodwardia Sm.

Wormia Rottb.

Wormskioldia

Thonn. et Sch.

Wrangelia $\mathrm{Ag}$.

Wredowia Eckl.

Wrirhtia $R B r$.

Wulfenia Jacq.

Wulffia Neck.

Wurmbea Thunb.

Wydleria $D C$.

Wylia 11 offm.

Xauthe $W$.

Xanthisma $D C$.

187
237

187

327

2268 Xylophagus $L k$.

161 Xylosteum Juss.

129 Xyris $L$.

187 Xysmalobium $R B r . \quad 160$

200

200 Yucca $L$.

Xanthium L. ? 90 (185)

Ximthocephalum $W .187$

Xanthoceras Bung. ? 28y

Xanthochymus Roxb. 302

Xanthocoina H. B. K. 187

Xantlıophyllum Roxb. 272
Xanthophytum Bl. 176

$\begin{array}{ll}\text { Xantlıophyllum Roxb. } & 272 \\ \text { Xanthophytum Bl. } & \mathbf{1 7 6}\end{array}$

Xanthorhiza Marsh.

326 Zapania Scop.
267

20

yloma Pers.

Xylomelum $S m$.

Xylomyzon Pers.

12 Xylophylla $L$.

74 Xylopia $P$. Br.

71 Xystidium Trin.

42

Fam.

? 66

200

36

229

4

187

187

\section{1}

129

184

42

293

221

71

72

46

187

187

? 300

? 72

26

129

265

4

306

5

3

5

5

3

4

113

5

5

96

330

178

48

66

302 Zacyntha Grertn. 188

87 Zahlbrucknera Rehb. 248

Zaleya Burm. $\quad 256$

Zaluzania Pers. $\quad 187$

Zamia $L$.

Zannichellia $L$. 32

Zanonia $L$.

195 
Zarabellia Cass.

Zea $\boldsymbol{L}$.

Zeocriton P. B.

Zeora $\mathrm{Fr}$.

Zepliyra Don.

Zephyranthes IIcrb.

Zerumbet $\boldsymbol{W}$ endl.

Zengites Schreb.

Zeuxinc $L d l$.

Zexmenia Llav.

Zeyheria Mart.

Zieria $S m$.
Fam.

187

42

42

27

66

69

76

42

78

187

133

278 Zollikofera Nees.

Zigadenus Mich.

Zilla Forsk.

Zingiber Gaertn.
Fom.

142 Zonaria $\mathrm{Ag}$.

71 Zornia Gm.

314 Zostera $L$.

Fam.

15 Zygnema Ag.

76 Zosterospe

Zycodon Hook.

32 Zygoglossum Reinu.

187 Zostcrostylis $\mathrm{Bl}$.

42 Zoysia $W$.

$2(10$ Zozimia Ilofjm.

142 Zucca Comm.

203 Zuccagnia Cav.

184 Zuccagnia Thunb.

222 Zuccarinia $B l$.

$18 \dot{8}$ Zwingera Schreb.
B. 43

78

42

20

200

195

222

6

177 Zythia $\mathrm{Fr}$.

280
66 Zyrphelis Coss.

Zygomence Salisb. 49

Zygopetalum Hook. $\quad 78$

Zygophyllum $L$. $\quad 276$

Zygostates Ldl. $\quad 28$

21

286

187

i) ruckfelier,

un deren Beriehtigung vor dem Gebrauche des

Clavi g gebeten wird:

S. 7. Z. 4. v. u. nach: rarius setze man: 3, 2 v.

S. 33. Z. 5. st. $2-3$-fill setze man: $2-5$-fid. 



<smiles>C1#CC2CC12</smiles> 


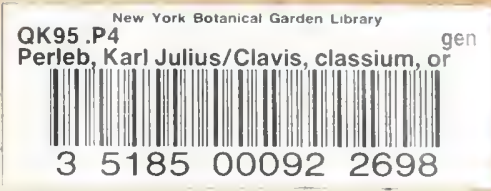




\section{$-x^{2}=5$}

a. 10

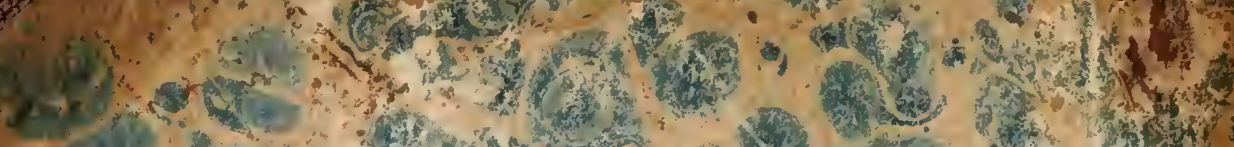

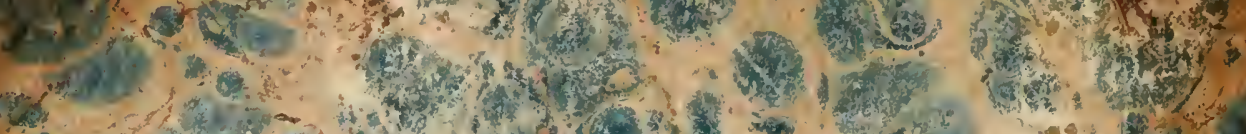

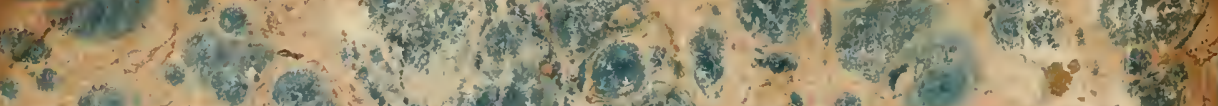

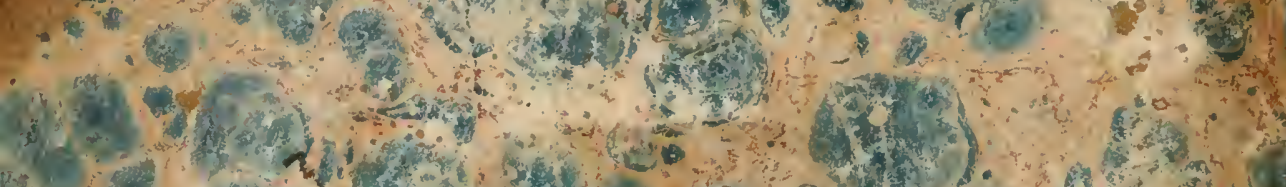

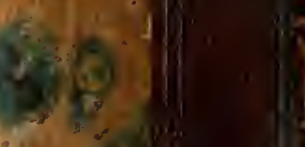

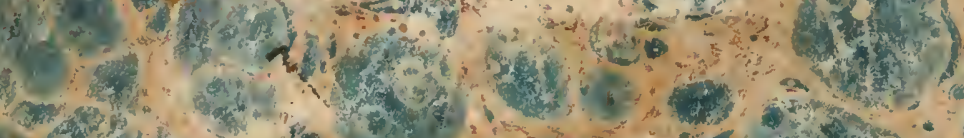

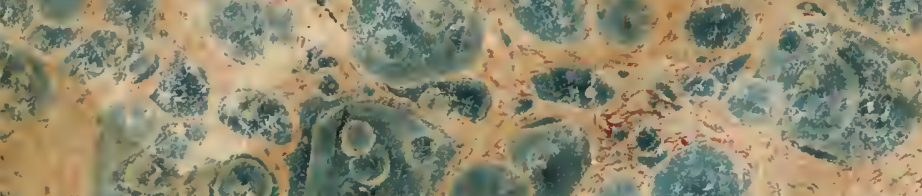

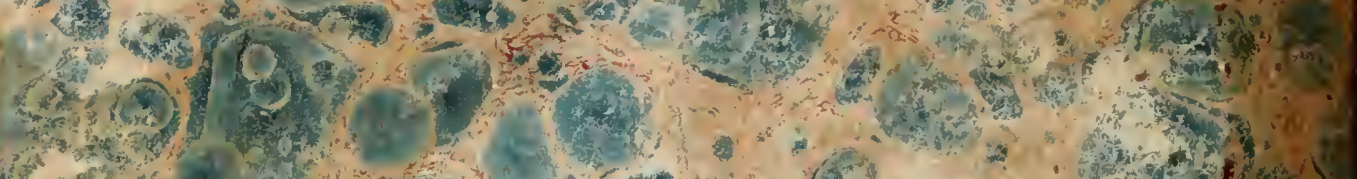

(1)

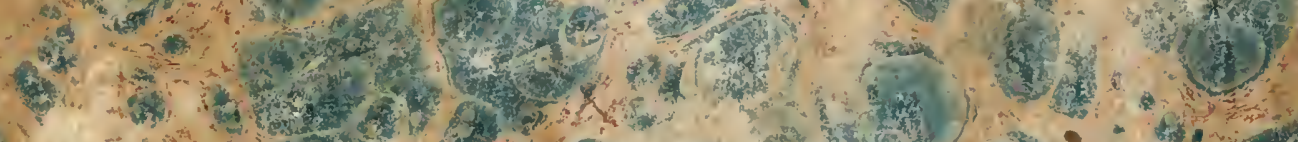

(1) (a) 1. 5. (4)

(n)

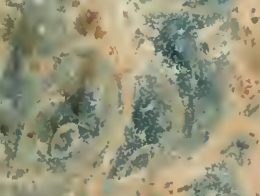

(3)

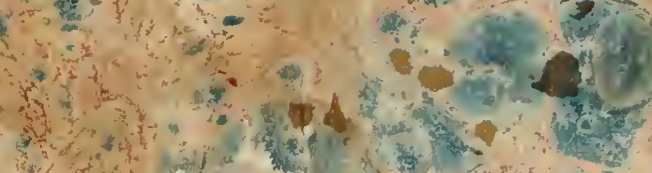

(1)

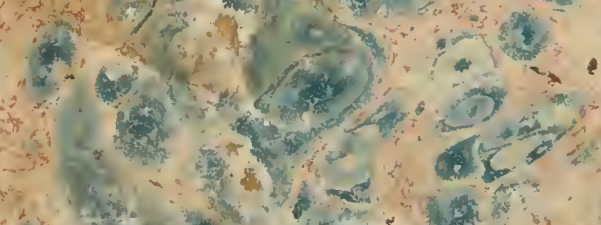

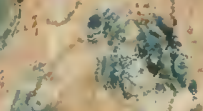

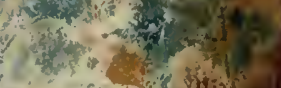

risely.

and

(5)

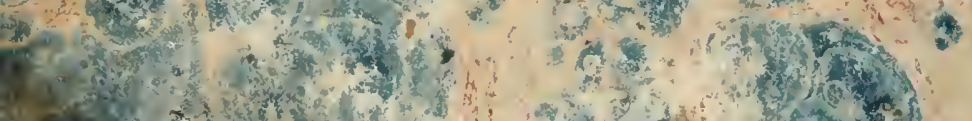

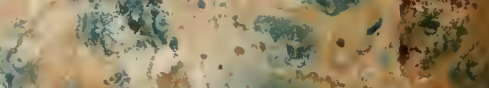

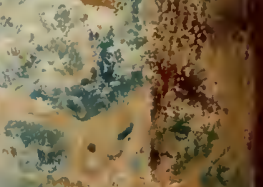

